\title{
Ein neues Konzept für die Optimierung intelligenter Aktoren im Bereich beweglicher elektrischer Verbraucher
}

\author{
Zur Erlangung des akademischen Grades eines \\ DOKTORS DER INGENIEURWISSENSCHAFTEN (Dr.-Ing.) \\ von der KIT-Fakultät für Maschinenbau des \\ Karlsruher Instituts für Technologie (KIT) \\ genehmigte \\ DISSERTATION
}

von

Dipl.-Ing. Friedrich Manz

Tag der mündlichen Prüfung:

Hauptreferent:

Korreferent:

Korreferent:
01.12 .2020

Prof. Dr.-Ing. habil. Georg Bretthauer Prof. Dr.-Ing. Veit Hagenmeyer Prof. Dr.-Ing. Ulrich Jumar 


\section{Markenrechtlicher Hinweis}

Die in dieser Arbeit wiedergegebenen Firmen-, Markennamen und Warenzeichen können auch ohne besondere Kennzeichnung geschützte Namen oder Marken sein und sind Eigentum des jeweiligen Herstellers. 


\section{Vorwort}

Die vorliegende Dissertation entstand während meiner Tätigkeit im zentralen Bereich Forschung und Entwicklung der SEW-Eurodrive GmbH. Die wissenschaftliche Betreuung erfolgte durch die Fakultät für Maschinenbau der Universität Karlsruhe (TH).

Ausdrücklich bedanken möchte ich mich bei Herrn Prof. Dr.-Ing. habil. Georg Bretthauer, Leiter des Instituts für Angewandte Informatik/Automatisierungstechnik, der die Betreuung seitens der Hochschule übernommen hat. Ohne seine hilfreiche Unterstützung wäre diese Arbeit nicht zustande gekommen.

Besonders bedanken möchte ich mich auch bei der Fa. SEW-Eurodrive GmbH, die mir die Erstellung dieser Arbeit im Rahmen des Doktorandenprogrammes ermöglichte. Meinen ganz besonderen Dank gilt dabei meinem Betreuer bei der SEW-Eurodrive, Herrn Dr.-Ing. Jochen Mahlein, der mir immer mit Rat und Tat zur Seite stand.

Weiterhin möchte ich mich bei allen Kollegen aus der Abteilung Innovation Mechatronik der SEW-Eurodrive GmbH für die stets angenehme Zusammenarbeit und freundschaftliche Arbeitsatmosphäre bedanken. Meinem besonderen Dank gilt Herrn Björn Egger und Herrn Volker Dietrich, die immer ein offenes Ohr für meine Probleme hatten und mich immer nach ganzer Kraft unterstützt haben.

Ohne studentische Mitwirkung wäre es nicht möglich gewesen, den gegenwärtigen Stand der Arbeit zu erreichen. Ich möchte daher allen Diplomanden und Praktikanten, die an dem Projekt mitgearbeitet haben, meinen herzlichen Dank aussprechen.

Nicht zuletzt gilt mein Dank meiner Ehefrau Gerda und meinem Sohn Johannes, die mich während der Zeit meiner Arbeit stets moralisch unterstützt haben und immer Verständnis hatten, wenn private Dinge zurückstehen mussten. 



\section{Kurzzusammenfassung}

Die Smart Factory, auch als intelligente Fabrik bezeichnet, steht als Synonym für die Schaffung von effektiven und flexiblen Produktionsumgebungen in der Industrie durch Digitalisierung und cyber-physische Systeme. Um Fertigungs- und Produktionsanlagen flexibel und effizient in den Produktionsablauf zu integrieren, spielt auch die Einbindung von mobilen Anwendungen im Bereich des Maschinenbaus und deren Komponenten eine wichtige Rolle. Ermöglicht wird die intelligente Fabrik u.a. auch durch die Verwendung kompakter mechatronischer Systeme und deren Teilkomponenten wie Sensoren, Aktoren und der dazugehörigen Mikroelektronik. Hier stellt sich die Frage, wie die Energiezuführung und die Datenanbindung flexibel zu solchen mobilen Anwendungen bzw. zu den intelligenten mechatronischen Systemen bereitgestellt werden kann.

Diese Dissertation liefert einen Beitrag zur Entwicklung eines intelligenten Aktors im Bereich der berührungslosen induktiven Energie- und Datenübertragung für mobile Anwendungen bzw. beweglichen elektrischen Verbrauchern. Das vorgestellte neue Konzept zeigt den Aufbau und die Realisierung des intelligenten Aktors. In einem ersten Schritt wird ein induktives kontaktloses Energieübertragungssystem an einem Funktionsmuster aufgebaut. Auf Grundlage einer OFDM-basierten Datenkommunikation und durch die Integration eines entwickelten Zeitstempelverfahrens sind eine Kommunikation und Synchronisation am Funktionsmuster über das kontaktlose Energieübertragungssystem möglich. Dabei wird auch gezeigt, dass sich die Taktfrequenz für die Synchronisierungsvorgänge bei der Übertragung von Daten zwischen den einzelnen Kommunikationsteilnehmern deutlich reduzieren lässt. Untersucht wird dabei die Ganggenauigkeit bzw. die Frequenzkonstanz von verschiedenen Oszillatorklassen. Im weiteren Verlauf der Arbeit werden der Entwurf und die Entwicklung einer Hardware- und Regelungsplattform für einen intelligenten Aktor gezeigt und erarbeitet. Innerhalb eines SoPC-basierten Embedded Systems wird ein kompaktes und skalierbares Motorsteuerkonzept für den Betrieb einer PMSM vorgestellt. Im letzten Teil der Arbeit wird die Entwicklung und die Umsetzung eines prädiktiven Regelungsverfahrens in einer durchgeführten Simulation vorgestellt. In der Simulation werden die Vorteile und die Leistungsfähigkeit der MPC-Regelung im Vergleich zu einer klassischen PID-Kaskadenregelung für das Stör- und Führungsverhalten für den Betrieb einer PMSM aufgezeigt. 



\section{Inhaltsverzeichnis}

1. Einleitung 1

1.1. Bedeutung der Arbeit . . . . . . . . . . . . . . . . . 1

1.1.1. Berührungslose Energieübertragung . . . . . . . . . . . 1

1.1.2. Smart Factory und intelligente Aktoren . . . . . . . . . . . . . 4

1.2. Darstellung des Entwicklungsstandes . . . . . . . . . . . . . 5

1.2.1. Berührungslose Datenkommunikation . . . . . . . . . . 5

1.2.2. Aktoren und mechatronische Systeme . . . . . . . . . . . 8

1.2.3. Intelligente Aktoren als Systemkomponenten . . . . . . . . . . 14

1.3. Ziele und Aufgaben . . . . . . . . . . . . . . . . . . 17

2. Neues Konzept für die Optimierung intelligenter Aktoren 21

2.1. Grundstruktur des neuen Konzeptes . . . . . . . . . . . . . . . . . . . 23

2.2. Übersicht und Beschreibung der Komponenten . . . . . . . . . . . . . 24

3. Aufbau eines Funktionsmusters mit kontaktloser Energieübertragung $\quad \mathbf{2 9}$

3.1. Komponenten der kontaktlosen Energieübertragung . . . . . . . . . . . 30

3.2. Etikettieranlagen in der Industrie . . . . . . . . . . . . . . . . . . . . 32

3.3. Funktionsmuster einer Etikettiermaschine . . . . . . . . . . . . . 34

3.4. Aufbau des Funktionsmusters . . . . . . . . . . . . . . . 36

3.4.1. Stationärer mechanischer Aufbau des Funktionsmusters . . . . 36

3.4.2. Beweglicher mechanischer Aufbau des Funktionsmusters . . . 38

3.4.3. Stationärer elektrischer Aufbau des Funktionsmusters . . . . . 38

3.4.4. Beweglicher elektrischer Aufbau des Funktionsmusters . . . . . 40

3.5. Übertragungskanal der kontaktlosen Energieübertragung . . . . . . . . 42

3.5.1. Eigenschaften des Übertragungskanals . . . . . . . . . . . . . . . 43

3.5.2. Störeinflüsse des Übertragungskanals . . . . . . . . . . . . . . . . . . 4 45

3.6. Zusammenfassung der Ergebnisse . . . . . . . . . . . . . . . 46

4. Datensynchronisierung über das kontaktlose Energieübertragungssystem 49

4.1. Synchronität und Gleichzeitigkeit . . . . . . . . . . . . . 50

4.1.1. IEEE-1588 - Precision Time Protocol . . . . . . . . . . . . . . 52

4.1.2. Moderne Feldbussysteme . . . . . . . . . . . . . . . . . 55

4.2. Schichtenmodell und Modulation . . . . . . . . . . . . . . . 57

4.2.1. Referenzmodell des Kommunikationssystems . . . . . . . . . 57 
4.2.2. Digitale Modulationsverfahren . . . . . . . . . . . . . . . . 59

4.2.3. Orthogonales Frequenzmultiplexverfahren . . . . . . . . . . 61

4.2.4. Aufbau des Datenübertragungssystems . . . . . . . . . . . . 63

4.3. Realisierung der Datenkommunikation . . . . . . . . . . . 66

4.3.1. Aufbau des Modems . . . . . . . . . . . . . . . . 67

4.3.2. Master-Slave-Betrieb . . . . . . . . . . . . . . . . . . 70

4.3.3. Parameter des Datenübertragungssystems . . . . . . . . . . 71

4.4. Darstellung des Verfahrens für die Synchronisierung der Teilnehmer . 73

4.4.1. Erweitertes Datentelegramm . . . . . . . . . . . . . . 74

4.4.2. Laufzeitermittlung im Sendepfad . . . . . . . . . . . . 75

4.4.3. Laufzeitermittlung im Empfangspfad . . . . . . . . . . . . 76

4.4.4. Ermittlung der Synchronisationsgenauigkeit . . . . . . . . . 78

4.4.5. Einfluss der Gangabweichung . . . . . . . . . . . . . . 80

4.5. Bestimmung der Ganggenauigkeit mit der Zwei-Oszillator-Methode . 81

4.5.1. Messaufbau zur Bestimmung der Gangabweichung . . . . . . . 82

4.5.2. Darstellung der Ergebnisse der Gangabweichung . . . . . . . . 83

4.5.3. Bestimmung der Allan-Varianz . . . . . . . . . . . . . 85

4.6. Zusammenfassung der Ergebnisse . . . . . . . . . . . . . . . . 87

5. Neue Hardware- und Regelungsplattform für intelligente Aktoren $\quad \mathbf{8 9}$

5.1. Mikrocontroller versus programmierbarer Hardware . . . . . . . . . . 90

5.2. Wahl einer geeigneten Referenzplattform . . . . . . . . . . . . . . . 92

5.2.1. Beschreibung der verwendeten Leistungsplatine . . . . . . . . 92

5.2.2. Beschreibung der verwendeten Steuerplatine . . . . . . . . . . 97

5.3. Darstellung der neuen Hardware- und Regelungsplattform . . . . . . 100

5.3.1. Permanentmagneterregte Synchronmaschine . . . . . . . . . 100

5.3.2. Koordinatentransformationen . . . . . . . . . . . . . . 103

5.3.3. Feldorientierte Regelung . . . . . . . . . . . . . . . . 107

5.3.4. Darstellung der Raumzeigermodulation . . . . . . . . . . . 117

5.3.5. Aufbau und Realisierung einer neuen Regelungsplattform innerhalb einer Embedded-System Umgebung . . . . . . . . . . 123

5.4. Zusammenfassung der Ergebnisse . . . . . . . . . . . . . . . . 144

6. Entwicklung eines Regelungskonzeptes mit prädiktiven Verhalten $\quad 147$

6.1. Darstellung modellprädiktiver Regelverfahren . . . . . . . . . . . . . 148

6.1.1. Dynamic Matrix Control . . . . . . . . . . . . . . . . 153

6.1.2. Generalized Predictive Control . . . . . . . . . . . . . . . . . . 155

6.2. Modellbildung der permanentmagneterregten Synchronmaschine . . . 157

6.2.1. Modell im statorfesten Koordinatensystem . . . . . . . . . . . 158

6.2.2. Modell im rotorfesten Koordinatensystem . . . . . . . . . . . 162

6.3. Bewertung der modellprädiktiven Regelung . . . . . . . . . . . . . . . 164

6.3.1. Modellbildung im Zustandsraum . . . . . . . . . . . . . 166 
6.3.2. Linear Model Predictive Control Algorithmus . . . . . . . . . 168

6.3.3. Parameter des modellprädiktiven Regelverfahrens . . . . . . . 169

6.3.4. Darstellung der Ergebnisse der modellprädiktiven Regelung . . 170

6.4. Zusammenfassung der Ergebnisse . . . . . . . . . . . . 178

$\begin{array}{ll}\text { 7. Zusammenfassung } & 181\end{array}$

$\begin{array}{ll}\text { Literaturverzeichnis } & 187\end{array}$

A. Technische Daten des Funktionsmusters 205

$\begin{array}{lr}\text { B. Lineare Oszillatoren } & 207\end{array}$

B.1. Wirkungsweise von Oszillatoren . . . . . . . . . . . . . . . . . . 207

B.2. Eigenschaften von Schwingquarzen . . . . . . . . . . . . . . 210

B.3. Beschreibung unterschiedlicher Oszillatorklassen . . . . . . . . . . . 217

C. Parameter und Kennwerte der Regelung 221

C.1. Modellparameter der Regelung . . . . . . . . . . . . . . . . . . . . . . 221

C.2. Kennwerte der Kaskadenregelung . . . . . . . . . . . . . . . . . . . 222

C.3. Modulblöcke der Regelstrecke . . . . . . . . . . . . . . . . . . . . . . 223

$\begin{array}{ll}\text { D. Bezeichnungen } & 225\end{array}$ 



\section{Abbildungsverzeichnis}

1.1. Induktives kontaktloses Energieübertragungssystem [182] . . . . . . . 3

1.2. Funkbasierte Kommunikation in einer Montagehalle [80]. . . . . . . . 7

1.3. Aufbau eines mechatronischen Systems [43]. . . . . . . . . . . . . . . 10

1.4. Darstellung eines Aktors mit Ein- und Ausgangsgröße [43]. . . . . . . 10

1.5. Aufbau eines einfachen elektromechanischen Aktors [43]. . . . . . . . 11

1.6. Aufbau eines piezoelektrischen Aktors [43]. . . . . . . . . . . . . . . . 11

1.7. Aufbau eines fluidtechnischen Aktors [43]. . . . . . . . . . . . . . . . 12

1.8. Aufbau eines thermomechanischen Aktors [43] . . . . . . . . . . . . . 13

1.9. Aufbau eines intelligenten Aktors am Beispiel eines Servoantriebes $[43],[84] \ldots \ldots \ldots \ldots \ldots$

2.1. Mobiles Assistenzsystem für die Getriebemontage [205] . . . . . . . . . 22

2.2. Aufbau des intelligenten Aktors. . . . . . . . . . . . . . . . . . 23

3.1. Aufbau eines kontaktlosen Energieübertragungsystems [182]. . . . . . 30

3.2. Aufbau des Anschaltmoduls [182] . . . . . . . . . . . . . . . 31

3.3. Darstellung einer industriellen Etikettiermaschine [114] . . . . . . . . 33

3.4. Aufgebautes Funktionsmuster einer Etikettiermaschine. . . . . . . . . 34

3.5. Bewegungsablauf beim Etikettiervorgang. . . . . . . . . . . . . . . . 35

3.6. Aufbau des stationären Teils des Funktionsmusters. . . . . . . . . . . 37

3.7. Montage des U-Übertragerkopfes am Funktionsmuster. . . . . . . . . 38

3.8. Blockschaltbild der stationären elektrischen Komponenten. . . . . . . 39

3.9. Aufbaubild Schaltschrank für die stationäre Seite. . . . . . . . . . . . 41

3.10. Blockschaltbild der beweglichen elektrischen Komponenten. . . . . . . 42

3.11. Dezentrales Modul für bewegliche elektrische Verbraucher. . . . . . . 43

3.12. Übertragungsverhalten des Datenkanals. . . . . . . . . . . . . . . . . 44

4.1. Gleichzeitigkeit und Synchronität [89]. . . . . . . . . . . . . . . . 51

4.2. IEEE-1588 Synchronisationstelegramme [53] . . . . . . . . . . . . . 54

4.3. OSI-Schichtenmodell [55]. . . . . . . . . . . . . . . . . 58

4.4. Signalraumkonstellation 16-QAM [230]. . . . . . . . . . . . . . 61

4.5. Spektrum der Unterträger mit Summenspektrum bei OFDM [80]. . . 62

4.6. Blockschaltbild des gesamten OFDM-Datenübertragungssystems [96]. 64

4.7. Datenkommunikation mit PLC-Modem. . . . . . . . . . . . . . 66 
4.8. Prinzipieller Aufbau des PLC-Modems. . . . . . . . . . . . . . . . . . 67

4.9. Layout des PLC-Modems [80] . . . . . . . . . . . . . . . . 69

4.10. SSC-Schnittstelle [80]. . . . . . . . . . . . . . . . . . . . . . . . 69

4.11. Schutzintervall gegen Intersymbol-Interferenz [80] . . . . . . . . . . 72

4.12. OFDM-Symboldauer [80]. . . . . . . . . . . . . . . . . 73

4.13. Aufbau und Struktur des Datentelegramms. . . . . . . . . . . . . . . 74

4.14. Aufzeichnung des Datentelegramms. . . . . . . . . . . . . . . . . . . 74

4.15. Laufzeitermittlung im Sendepfad. . . . . . . . . . . . . . . . 76

4.16. Laufzeitermittlung im Empfangspfad. . . . . . . . . . . . . . . . . . . . 77

4.17. Synchronisation zwischen Master- und Slave-Modem. . . . . . . . . . 78

4.18. links: OCXO-Platine (Oberseite), mitte: OCXO-Platine (Unterseite), rechts: TCXO-Platine. . . . . . . . . . . . . . . 82

4.19. Messaufbau zur Bestimmung der Gangabweichung. . . . . . . . . . . 83

4.20. Gangabweichung des Standard-Oszillators. . . . . . . . . . . . . . . . 84

4.21. Gangabweichung des TCXO-Oszillators. . . . . . . . . . . . . . . 85

4.22. Gangabweichung des OCXO-Oszillators. . . . . . . . . . . 86

4.23. Übersicht der Allan-Varianz aller drei Oszillatoren. . . . . . . . . . . 87

5.1. Blockdiagramm für den Leistungsteil des Umrichters [49] . . . . . . . 93

5.2. Leistungsteil des Umrichters [49]. . . . . . . . . . . . . . . . . . . . 94

5.3. PFC-Modul mit EMV-Filter [49]. . . . . . . . . . . . . . . . . . . . . . 94

5.4. Aufbau Schaltnetzteil [49]. . . . . . . . . . . . . . . . . . . . . 95

5.5. Ansteuerung der IGBT-Endstufe [49] . . . . . . . . . . . . . . . . 96

5.6. Steuerteil Umrichter [48]. . . . . . . . . . . . . . . . . . . . . . . 98

5.7. Blockdiagramm DBC3C40 Development Board [48] . . . . . . . . . . 99

5.8. Modell der PMSM mit Oberflächenmagneten [190]. . . . . . . . . . . 102

5.9. Übergang vom Dreiphasensystem in das $\alpha, \beta$-Koordinatensystem [181]. 103

5.10. Zusammenhang zwischen $\alpha, \beta$ - und $d, q$-Koordinatensystem [181]. . . 106

5.11. Feldorientierte Regelung der PMSM [181]. . . . . . . . . . . . . . . 108

5.12. Übersicht Transformationen [181] . . . . . . . . . . . . . . . . . . . . 109

5.13. Blockschaltbild der Stromregelung mit Entkopplung [181]. . . . . . . 111

5.14. Vereinfachter Stromregelkreis mit Ersatzzeitkonstante [181]. . . . . . 112

5.15. Vereinfachter Drehzahlregelkreis mit Ersatzzeitkonstante [181] . . . . 113

5.16. Drehzahlregelkreis mit Beschleunigungs-Vorsteuerung [127] . . . . . . 115

5.17. PI-Regler mit Anti-Windup Struktur [190]. . . . . . . . . . . . . . . 116

5.18. Aufbau eines Spannungszwischenkreis-Umrichters [189] . . . . . . . . 117

5.19. Prinzipschaltbild des U-Wechselrichters [91]. . . . . . . . . . . . . 118

5.20. Raumzeigerdarstellung der Ausgangsspannungen [91] . . . . . . . . . 120

5.21. Randvektoren des Spannungsraumzeigers [91]. . . . . . . . . . . . . 120

5.22. Bildung des Spannungsvektors [91]. . . . . . . . . . . . . . . . . 121

5.23. Blockschaltbild eines $\Sigma \Delta$-Modulator 1. Ordnung [21] . . . . . . . . . 125 
5.24. Abhängigkeiten des $\Sigma \Delta$-Modulator von OSR und Ordnungsgrad (Darstellung der Ergebnisse aus Gl. (5.52)). . . . . . . . . . . . . . . 128

5.25. $\Sigma \Delta$-Wandler: Modulator mit Digitalteil [146]. . . . . . . . . . . . . . 128

5.26. Digitales Filter 3. Ordnung [146]. . . . . . . . . . . . . . . 130

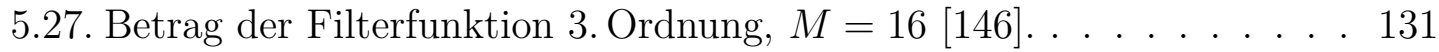

5.28. 32 Bit RISCS HDL-Struktur auf einem SoPC [6]. . . . . . . . . . . . 132

5.29. Entwicklungsumgebung des SoPC-Systems [6] . . . . . . . . . . 133

5.30. Designschritte zum Aufbau des SoPC-Systems [6] . . . . . . . . . . . 136

5.31. Custom Instruction [7]. . . . . . . . . . . . . . . . . . . . 137

5.32. Aufbau und Komponenten der Motorsteuerung. . . . . . . . . . . . . 138

5.33. Blockschaltbild der Motorsteuerung. . . . . . . . . . . . . . . 139

5.34. FPGA-basierte Positionsregelung. . . . . . . . . . . . . . . . . . . 145

6.1. Struktur einer prädiktiven Regelung [167]. . . . . . . . . . . . . . . . 149

6.2. Prinzipielle Funktionsweise der prädiktiven Regelung [167] . . . . . . . 150

6.3. Stator- und Rotorkoordinatensystem der PMSM [181]. . . . . . . . . 159

6.4. Signalflussplan der PMSM im statorfesten Koordinatensystem [190]. . 162

6.5. Signalflussplan der PMSM im rotorfesten Koordinatensystem [190]. . 165

6.6. Blockschaltbild für die Simulation der Kaskadenregelung. . . . . . . . 171

6.7. Blockschaltbild Simulation MPC-Regelung. . . . . . . . . . . . . . . . 173

6.8. Vergleich Führungsverhalten LMPC/PID für kleine Drehzahlen. . . . 174

6.9. Vergleich Führungsverhalten LMPC/PID für große Drehzahlen. . . . 175

6.10. Vergleich Störverhalten LMPC/PID für kleine Drehzahlen. . . . . . . 176

6.11. Vergleich Störverhalten LMPC/PID für große Drehzahlen. . . . . . . 177

B.1. Rückgekoppelte Verstärkerschaltung [226]. . . . . . . . . . . . . . . . 208

B.2. Schnittlage der Quarzplättchen im Kristall [77]. . . . . . . . . . . . . 211

B.3. Vergleich Temperaturverlauf bei AT- und SC-Schnitt [143] . . . . . . . 212

B.4. Darstellung der Allan-Varianz [143] . . . . . . . . . . . . . . . . 215

B.5. Frequenzspektrum eines Oszillators [143] . . . . . . . . . . . . . 215

B.6. Ersatzschaltbild eines Schwingquarzes [143]. . . . . . . . . . . . 216

C.1. Blockschaltbild des nichtlinearen Zustandsraummodells. . . . . . . . . 223

C.2. Blockschaltbild der Spannungsbegrenzung. . . . . . . . . . . . . . . . 224 



\section{Tabellenverzeichnis}

4.1. Daten- und Konfigurationsregister [80] . . . . . . . . . . . . . . . . . 70

4.2. Control-Register im Steuerkanal [80] . . . . . . . . . . . . . . . . 71

4.3. Messintervalle zur Bestimmung der Allan-Varianz . . . . . . . . . . 86

5.1. Spannungsraumzeiger des Wechselrichters [91] . . . . . . . . . . . . 119

5.2. Randkomponenten des Spannungsvektors [181]. . . . . . . . . . . . . 123

5.3. Werte des $\operatorname{sinc}^{3}$-Filters in Abhängigkeit von $M$ und $f_{\Sigma \Delta}$ [146], [235]. 144

B.1. Klassen von Oszillatoren [143], [164]. . . . . . . . . . . . . . . 218 



\title{
Abkürzungsverzeichnis
}

\author{
A/D Analog/Digital \\ AC Alternating Current \\ AFE Analog Front End \\ ALU Arithmetic Logic Unit \\ AP Access Point \\ API Application Program Interface
}

ARIMA Auto-Regressive Integrating Moving-Average

ASF Avalon Switch Fabric

ASIC Application-Specific Integrated Circuit

ASK Amplitude Shift Keying

ASM Asynchronmaschine

AT Acknowledge Telegramm

BLAC Brushless Alternating Current

BLDC Brushless Direct Current

BMC Best Master Clock

CAN Controller Area Network

CARIMA Controller Auto-Regressive Integrated Moving-Average

CCM Continous Conduction Mode 
CI Custom Instructions

CPLD Complex Programmable Logic Device

CPS Cyber-Physisches System

CPU Central Prozessing Unit

D/A Digital/Analog

DAB Digital Audio Broadcasting

DC Direct Current

DDS Direct Digital Synthesis

DFT Diskrete Fourier-Transformation

DMC Dynamic Matrix Control

DQPSK Differential Quaternary Phase Shift Keying

DSC Digitaler Signal-Controller

DSP Digitaler Signal-Prozessor

DSS Dickenscherungsschwinger

DTC Direct Torque Control

DVB Digital Video Broadcasting

EHB Elektrohängebahn

EMV Elektromagnetische Verträglichkeit

ERP Enterprise Resource Planning

FDM Frequency-Division Multiplexing

FFT Fast Fourier-Transformation

FIR Finite Impulse Response

FM Funktionsmuster 
FOC Field Oriented Control

FPGA Field Programmable Gate Array

FPU Floating Point Unit

FSK Frequency Shift Keying

FTS Fahrerloses Transportsystem

GP General Purpose

GPC Generalized Predictive Control

GTO Gate Turn Off Thyristor

HS-SSC High Speed-Synchronous Serial Channel

$\mathrm{I}^{2} \mathrm{C} \quad$ Inter-Integrated Circuit

I/O Input/Output

IDE Integrated Design Environment

IDFT Inverse Diskrete Fourier-Transformation

IEC International Electrotechnical Commission

IEEE Institute of Electrical and Electronics Engineers

IFFT Inverse Fast Fourier-Transformation

IGBT Insulated Gate Bipolar Transistor

IIR Infinite Impulse Response

IP Intellectual Property

IRQ Interruptcontroller

ISI Intersymbol-Interferenzen

JTAG Joint Test Action Group

LAN Local Area Network 


$\begin{array}{ll}\text { LE } & \text { Logikelemente } \\ \text { LMPC } & \text { Linear Model Predictive Control } \\ \text { LSB } & \text { Least Significant Bit } \\ \text { LVDS } & \text { Low Voltage Differential Signaling } \\ \text { MAC } & \text { Media Access Control Layer } \\ \text { MDT } & \text { Master Data Telegramm } \\ \text { MIMO } & \text { Multi-Input Multi-Output } \\ \text { MIPS } & \text { Million Instructions Per Second } \\ \text { MPC } & \text { Model Predictive Control } \\ \text { MST } & \text { Master-Synchronisations Telegramm } \\ \text { NB } & \text { Nebenbedingungen } \\ \text { NIC } & \text { Negative Impedance Converter } \\ \text { NTC } & \text { Negative Temperature Coefficient Thermistor } \\ \text { OCXO } & \text { Oven Controlled Crystal Oscillator } \\ \text { OFDM } & \text { Orthogonal Frequency Division Multiplexing } \\ \text { OOK } & \text { On/Off Keying } \\ \text { OST } & \text { Open Systems Interconnection } \\ \text { Otine-Communication }\end{array}$


PLL Phase Locked Loop

PM Permanentmagnet

PMSM Permanent-Magnet Synchronous Motor

ppb parts per billion

ppm parts per million

PSK Phase Shift Keying

PTP Precision Time Protocol

PWM Pulsweitenmodulation

PXO Package Crystal Oscillator

QASK Quadrature Amplitude Shift Keying

QPSK Quadrature Phase Shift Keying

RISC Reduced Instruction Set Compute

RTC Real-Time Channel

SC Stress-Compensated

SERCOS Serial Real-time Communication System

SISO Single-Input Single-Output

SM Synchronmaschine

SMD $\quad$ Surface Mounted Devices

SNR Signal-to-Noise Ratio

SoPC $\quad$ System on a Programmable Chip

SPI Serial Peripheral Interface

SPS Speicherprogrammierbare Steuerung

SRAM Static Random Access Memory 
SSC Synchronous Serial Channel

STD Synchronous Time Division

STO Safe Torque Off

TB Time Base

TCXO Temperature Compensated Crystal Oscillator

TFH Trägerfrequenztechnik auf Hochspannungsleitungen

TFT Thin-Film Transistor

TRT Tonfrequenz-Rundsteuertechnik

TTM Time To Market

UCC Unified Communication Channel

VCXO Voltage Controlled Crystal Oscillator

VHDL Very High Speed Integrated Circuit Hardware Description Language

WLAN Wireless Local Area Network

ZK Zwischenkreis

ZRM Zustandsraummodell 


\section{Einleitung}

\subsection{Bedeutung der Arbeit}

Die elektrische Energie ist als leitungsgebundener Energieträger eine der wichtigsten Energiearten für die Versorgung von Verbrauchern mit Nutzenergie. Stationäre, also feststehende Verbraucher, können hier einfach, in der Regel durch Kupferkabel, an vorhandene Verteilerstationen zur Energieversorgung angeschlossen werden.

Im Bereich der mobilen Intralogistik und angrenzenden Gebieten des klassischen Maschinenbaus, in denen bewegliche bzw. mobile elektrische Verbraucher mit Energie versorgt werden müssen, stellt sich die grundlegende Frage der Energiezuführung und Datenanbindung zu den mobilen Komponenten.

Die Frage stellt sich auch immer stärker im Bereich der Industrie 4.0, wo die Flexibilität des Produktionsumfeldes und die Verfügbarkeit der Anlagen und Maschinen im Fokus stehen. Die intelligente Fabrik, auch als Smart Factory bezeichnet, steht dabei als Synonym für die fortschreitende Digitalisierung und den Einsatz von cyberphysische Systemen.

Um solche gewünschte flexible Prozesse im Produktionsumfeld abzubilden, sind intelligente Sensoren und Aktoren wichtige zentrale Bestandteile. Die vorliegende Arbeit geht daher der Frage nach, wie die Energiezuführung und Datenanbindung flexibel zu mobilen technischen Systemen bereitgestellt werden kann und wie ein intelligenter Aktor in einem solchen technischen Umfeld zu realisieren ist.

\subsubsection{Berührungslose Energieübertragung}

In der Regel wurden bzw. werden für die Versorgung der beweglichen elektrischen Verbraucher mit Energie und Daten überwiegend Systeme eingesetzt, die mit Schleifringen oder Energieketten arbeiten. Schleifringe unterliegen einem permanenten Verschleiß, d.h. sie sind nicht wartungsfrei, sie sind verschmutzungsanfällig und sie müssen mechanisch genau geführt werden. Energieketten unterliegen gleichfalls mechanischen Beanspruchungen sowohl bei den mechanisch sich aufeinander reibenden 
Kettengliedern, als auch bei den notwendigen eingesetzten hochflexiblen Kabelverbindungen. Weiterhin werden Grenzen aufgezeigt bei langen oder gekrümmten Fahrwegen oder bei Abläufen, in denen eine hohe Dynamik des Bewegungsvorganges vorausgesetzt wird. Gleichfalls gibt es Einschränkungen bei Applikationen, in denen sich bewegliche elektrische Verbraucher kreuzen.

Bei frei fahrenden oder zumindest spurgeführten Systemen war hier in der Vergangenheit nur der Einsatz batteriegestützter Konzepte realisierbar. Batterien haben jedoch den Nachteil, dass die Lebensdauer und der verfügbare Energieinhalt begrenzt sind. Bei hohem benötigten Energiegehalt der Batterie müssen das Eigengewicht der Batterie und der dafür benötigte große Bauraum konstruktiv berücksichtigt werden. Klassische Bleiakkumulatoren weisen dabei eine hohe Ladezeit der Batteriezelle aus, was die Verfügbarkeit der Anwendungen, z.B. bei gefordertem Dreischichtbetrieb, zusätzlich einschränkt.

Andere Akkumulatortypen, wie z.B. Lithium-Ionen-Akkumulatoren, verfügen über eine höhere massenbezogene spezifische Energie, sie benötigen jedoch in der Regel eine elektronische Überwachungsschaltung, da die Zellen auf Tiefentladung und Überladung empfindlich reagieren. Gerade bei der Überladung der Zellen reagieren die meisten Lithium-Ionen-Akkumulatorentypen empfindlich auf Übertemperatur. Für die Handhabung und Beförderung gelten, bedingt durch die hohe Brandgefahr, besondere Sicherheitsvorschriften.

Eine andere Möglichkeit, bewegliche elektrische Verbraucher mit Energie zu versorgen, kann durch den Einsatz kontaktloser Energieübertragungssysteme, die nach dem Prinzip der elektromagnetischen Induktion arbeiten, realisiert werden (vgl. [2], [83], [106], [115], [166], [173]). Auf der primären oder auch stationären Seite stellt in der Anwendung ein fest verlegter Linienleiter - im Boden, in einer Verlegeplatte oder in einer Profilleiste montiert - die Einspeiseenergie zur Verfügung. Ein konstanter eingeprägter Wechselstrom, der durch den Linienleiter fließt, induziert dabei auf der mobilen oder beweglichen Seite in einer Sekundärspule, dem sogenannten Übertragerkopf, eine Spannung, die dann den mobilen Komponenten zur Verfügung gestellt wird.

Abbildung 1.1 verdeutlicht das Funktionsprinzip noch einmal genauer. Ein netzseitig versorgter Einspeise-Steller [1] liefert zuerst die Ausgangsspannung $U_{A S}$. Der nachfolgende Gyrator [2] und der Anpass-Transformator [3] prägen den konstanten Wechselstrom $I_{L}$ im Linienleiter [4] ein. Über den Übertragerkopf [6] und den nachgeschalteten Anpass-Steller [7] werden die nachfolgenden Verbraucher, hier ein Frequenzumrichter [8] mit Motor [9], mit einer konstanten Zwischenkreisspannung $U_{Z K}$ versorgt.

Das Verfahren arbeitet vereinfacht ausgedrückt wie ein Transformator, der auf der Primärseite nur eine Windung besitzt und dessen Eisenkern nicht vollständig ge- 


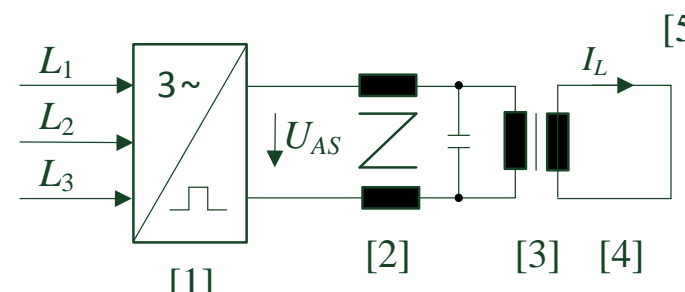

[1]
[2]
$[5]$

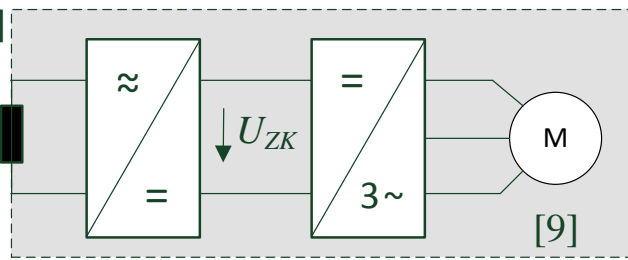

[6]
[7]
[8]

Abbildung 1.1.: Induktives kontaktloses Energieübertragungssystem [182]. [1] Einspeise-Steller, [2] Gyrator, [3] Anpass-Transformator, [4] Linienleiter, [5] Luftspalt, [6] Übertragerkopf, [7] Anpass-Steller, [8] Frequenzumrichter, [9] Fahrzeug bzw. Verbraucher.

schlossen ist. Die magnetischen Feldlinien werden auf der Primärseite über Luftwege geschlossen und auf der Sekundärseite durch die im Übertragerkopf integrierten Ferrite geführt. Bedingt durch die nicht vermeidbare große Streuung des magnetischen Feldes und der vorhandenen geringen Hauptinduktivität weist der Aufbau dadurch eine eher ungünstige Übertragungseigenschaft auf.

Verbessert werden können die Übertragungseigenschaften durch die Parallelschaltung eines Kondensators in der Sekundärspule. Wird der so entstehende Zweipol an seiner Resonanzfrequenz betrieben, erhöht sich dadurch die Impedanz der Anordnung maßgeblich und die an ihr abfallende nutzbare Spannung gleichermaßen. Durch eine solche Vorgehensweise wird die Übertragungseigenschaft bezüglich der Energieübertragung deutlich verbessert. Der konkrete Aufbau eines induktiven kontaktlosen Energieübertragungssystems wurde in der vorliegenden Arbeit realisiert und ist in Abschnitt 3.1 detailliert beschrieben.

Das Verlegen des Linienleiters entlang der Verfahrstrecke stellt zwar einerseits einen bestimmten Installationsaufwand dar, auf der anderen Seite arbeitet das System wartungsfrei und versorgt die mobilen bzw. beweglichen Verbraucher permanent mit Energie, auch bei benötigten höheren Leistungen der Endverbraucher. Gleichzeitig ist der Aufbau unempfindlich gegen Verschmutzung und zeichnet sich durch einen hohen Schutzgrad aus. Neben einfachen geraden Prozessstrecken und rotativen Bewegungsabläufen lassen sich auch Kreuzungspunkte zwischen mobilen Teilnehmern abbilden. Auch hochdynamische Bewegungen lassen sich problemlos realisieren.

Die Verwendung der Bezeichnung „,beweglicher elektrischer Verbraucher" in der vorgelegten Arbeit unterliegt keiner bekannten spezifischen Darstellung oder Definition in der Literatur. Er wird u.a. in [39], [197] verwendet. In der Arbeit bezieht sich die Verwendung des Begriffes auf Anwendungen innerhalb eines begrenzten Aktionsbereiches einer Maschine oder eines Anlagenteils, wo elektrische Verbraucher permanent über ein berührungsloses Energieübertragungssystem mit Energie versorgt werden. 
Innerhalb des Anwendungsbereiches können zusätzliche segmentartige Strukturen aufbaut werden.

Der Begriff soll sich dahingehend unterscheiden gegenüber der Definition eines „mobilen elektrischen Verbrauchers" im Sinne von „frei beweglich oder freifahrend“ wie z.B. bei Anwendungen im Bereich der Fahrerlosen Transportsysteme (FTS) [60], [66], [192], [193] oder mobilen Assistenzsysteme [32], [64], [205], [227], bei denen keine prinzipiellen räumlichen Einschränkungen betreffend des Aktionsbereiches bestehen. In solchen Anwendungen werden Fahrzeuge z.B. über Energiespeicher wie Batterien oder schnellladende DSK-Systeme (Doppelschichtkondensatoren) entweder vollständig autark oder zumindest teilweise durch den internen Energiespeicher mit Energie versorgt. Die teilweise bzw. ersatzweise Energieversorgung der Fahrzeuge erfolgt dann, wenn keine Ladestation oder eine externe Energieeinspeisungsstrecke im aktuellen Aktionsbereich der Applikation zur Verfügung steht.

\subsubsection{Smart Factory und intelligente Aktoren}

Die Smart Factory [78], [206], [210], auch als intelligente Fabrik bezeichnet, steht als Synonym für die Schaffung von effektiven Produktionsumgebungen in der Industrie durch Digitalisierung und cyber-physische Systeme (CPS) [11], [26], [51], [225]. Solche cyber-physischen Systeme, die auch als Entwicklungen im Bereich der Industrie 4.0 [219], [224] oder als das Industrielle Internet der Dinge (IIoT, engl.: Industrial Internet of Things) [29], [98], [209] bezeichnet werden, versprechen neue Maßstäbe der wirtschaftlichen Effizienz, Verfügbarkeit und Flexibilität im Produktionsumfeld.

IIoT verfolgt den Ansatz einer weitreichenden weiterführenden industriellen Revolution, in der Computer, Maschinen, Anlagen und deren Komponenten vermehrt miteinander kommunizieren und interagieren sollen. Es soll aber auch die Möglichkeit bestehen, durch einfache Interaktion zwischen Mensch und Maschine (MMS, MenschMaschine-Schnittstelle oder engl.: HMI, Human Machine Interface) Produktions-, Logistik- und Montageabläufe einfacher und effizienter zu gestalten und zu realisieren. Dadurch soll auch die zunehmende Komplexität der Produkte und Arbeitsabläufe, gerade bei der Produktion von Produkten mit einer großen Varianz aber kleinen Stückzahlen, vereinfacht und verbessert werden - auch im Sinne eines One-Piece-Flow [14].

Fertigungs- und Produktionsanlagen sollen verstärkt flexibel und schnell den aktuellen Bedürfnissen der Produktion angepasst werden. Die Maschinen und Geräte verfügen über eine eigene Intelligenz, sind mit dem übergeordneten Leitsystem aber auch mit anderen Maschinen und Geräten vernetzt und besitzen die Fähigkeit, relevante Daten mit allen am Produktionsprozess beteiligten Teilnehmern auszutauschen. 
Die Vernetzung zwischen den Komponenten führt zu mehr Prozessintelligenz und Prozesssicherheit.

Ermöglicht wird die intelligente Fabrik auch dadurch, dass Sensoren, Aktoren und die dazugehörige Mikroelektronik immer kleiner und günstiger hergestellt werden, und für die Kommunikation dieser Komponenten untereinander eine ausreichende Breitbandverbindung zur Verfügung steht, über die große Datenmengen schnell und effizient übertragen werden können.

Solchen intelligenten Maschinen, wie z.B. Service-Roboter [75], [125], [188], mobile Assistenzsysteme [32], [64], [205], [227] bzw. Fahrerlose Transportsysteme [60], [66], [192], [193], [220] oder auch intelligente Agenten [65], [90], [93], [134], [233] ist es möglich, selbstständig Fertigungs- und Montageprozesse zu koordinieren. Um solchen Arbeitsabläufen und Anforderungen gerecht zu werden, ist die Einbindung von intelligenten Aktoren von zentraler Bedeutung. Solche intelligenten Komponenten tragen damit ihren Beitrag zum IIoT bei.

\subsection{Darstellung des Entwicklungsstandes}

Neben der beschriebenen induktiven kontaktlosen Energieübertragung, die bereits als industrielles Produkt bei verschiedenen Herstellern [153], [197] seit vielen Jahren am Markt verfügbar ist, sollte natürlich auch die Datenkommunikation zwischen den Teilnehmern auf der stationären und der mobilen bzw. beweglichen Seite kontaktlos erfolgen.

In diesem Abschnitt werden zuerst die Möglichkeiten einer berührungslosen Datenübertragung bzw. Datenkommunikation im industriellen Umfeld dargestellt. Im weiteren Verlauf werden die Eigenschaften von einfachen und intelligenten Aktoren erläutert. Es soll aufgezeigt werden, wie sich Aktoren mit „intelligenten Funktionen“ innerhalb eines mechatronischen Systems einordnen lassen [24], [43], [84], [88].

\subsubsection{Berührungslose Datenkommunikation}

Berührungslose oder auch drahtlose Kommunikationssysteme finden in der Industrie immer größere Verbreitung. Aus dem Bereich der Industrie 4.0 werden hinsichtlich Flexibilität und Effizienz im Produktionsumfeld, Verfügbarkeit und intelligenter Vernetzung hohe Anforderungen gestellt. Für die technische Umsetzung der berührungslosen Datenkommunikation gibt es je nach Anwendungsfall vielfältige Möglichkeiten (vgl. [20], [50], [79]). 
Bei Rotationsbewegungen, d.h. einem stehenden und einem drehenden Maschinenteil, wie z.B. bei einer Flaschen-Abfüllanlage, kann für die Kommunikation zwischen beiden Maschinenkomponenten ein optischer Drehübertrager [237] eingesetzt werden. Für die Datenkommunikation bei liniengeführten Anwendungen, wie z.B. bei einem Regalbediengerät, eignen sich Datenlichtschranken [123]. Für frei im Raum fahrende oder spurgeführte mobile Systeme [99], [118] sind solche technischen Lösungen allerdings nicht möglich oder schwer umsetzbar.

\section{Verwendung von Wireless Local Area Network}

Eine Möglichkeit, flexibel unterschiedliche Teilnehmer über einen Kommunikationskanal miteinander zu verbinden, bietet der Aufbau einer Funkverbindung zwischen den stationären und mobilen Seiten oder den mobilen Teilnehmern untereinander. Immer größere Verbreitung im industriellen Umfeld findet der IEEE-802.11(x) Wireless Local Area Network-Standard (WLAN). Leistungsparameter wie Durchsatz, Latenz, Jitter und Frame-Verlust wurden in den neuen Standards immer weiter verbessert [44].

Auch wenn eine Funkverbindung bei der ersten Betrachtung durch ihre Flexibilität viele Vorteile mit sich bringt, sind bei der Umsetzung des Funknetzwerkes einige Punkte zu beachten. Oftmals sind für konkrete Anwendungen Echtzeit-Anforderungen für die Datenkommunikation mit garantierten Antwortzeiten zwischen den Teilnehmern gefordert. Anders als bei klassischen Bussystemen verfügt WLAN noch nicht über solche deterministischen Strukturen. Die Frequenzbänder sind weltweit nicht überall zugelassen. Die Frequenzen bzw. die freizuschaltenden Frequenzkanäle müssen mit dem Kunden für die konkrete umzusetzende Applikation abgeklärt werden, da sie sich unter Umständen mit der Infrastruktur des Kundennetzes überschneiden.

Auch ein Routing muss definiert sein. Bei großen Anlagen oder Montagehallen müssen die einzelnen Bereiche ausgeleuchtet und vermessen werden, um die Reflexionseigenschaften der Hallenstruktur zu berücksichtigen. So können großflächige Metallteile zu Reflexionen und Abschattungen führen, was die Sende- und Empfangseigenschaften einschränken kann. Abbildung 1.2 zeigt ein einfaches Beispiel dafür, dass durch eine falsche oder zu geringe Ausleuchtung der Hallenstruktur bzw. fehlender zusätzlicher Access-Points eine zuverlässige Kommunikation im Bereich B, C und D in der Montagehalle eingeschränkt oder gar nicht möglich ist. 


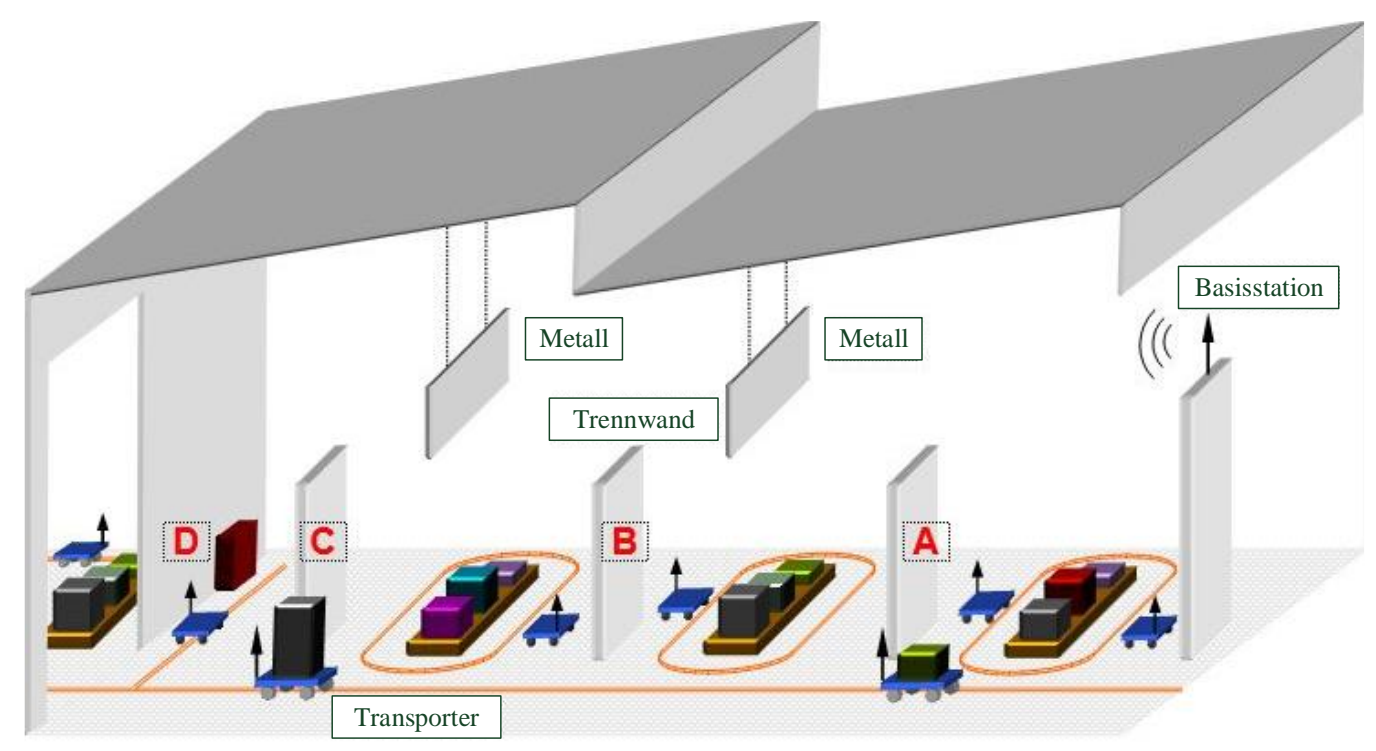

Abbildung 1.2.: Funkbasierte Kommunikation in einer Montagehalle [80].

\section{Einsatz von Leckwellenleiter}

Eine weitere Möglichkeit, eine kontaktlose Kommunikation zwischen mobilen Teilnehmern aufzubauen und obige Probleme der Abschattung und Reflexion zu vermeiden, bieten Schlitzkabel. Solche Schlitzkabel [111], sie werden auch als Leckkabel bezeichnet, sind elektrische Leckwellenleiter, bei denen ein verlegter Koaxialleiter als "Antenne" fungiert und dadurch ebenfalls WLAN-Signale übertragen werden.

Schlitzkabel, wie sie in der Hochfrequenztechnik verwendet werden, unterscheiden sich dadurch, dass entlang der Schirmung des Koaxialleiters kleine Schlitze oder Öffnungen, wie der Name schon sagt, eingestanzt sind. Entlang der Koaxialleitung kann sich so ein radialsymmetrisches Funkfeld ausbilden. Da das Funkfeld entlang des „undichten“ Koaxialkabels nur eng begrenzt ist, was einerseits gewollt ist, darf der Abstand der Leseantenne des mobilen Teilnehmers zum Schlitzkabel bestimmte Toleranzen nicht überschreiten. Bei Fahrerlosen Transportsystemen oder Elektrohängebahnen (EHB) [99], [118] entsteht dadurch ein erheblicher Installationsaufwand. Der Ausbau ist streckenabhängig, eine freie Navigation ist nicht möglich. Problematisch und aufwändig sind auch die Realisierung von Weichen oder Kreuzungsbereichen [99], [118].

\section{Nutzung der Powerline Communication}

Im Bereich der kontaktlosen Energieübertragung besteht die Möglichkeit, die vorhandene Leitung für die kontaktlose Energieübertragung gleichzeitig auch als Kommunikationskanal zu nutzen. Die Informationsübertragung über Energieversorgungsnetze existiert bereits seit Ende des 19. Jahrhunderts. Mittels Frequenzgeneratoren wurden 
Frequenzen generiert und dem vorhandenen Gleichstromnetz überlagert [100]. Im Jahr 1920 wurde die Trägerfrequenztechnik auf Hochspannungsleitungen (TFH) verwendet, um Informationen zwischen verbundenen Kraftwerken auszutauschen [160], [174]. Etwas später, im Jahr 1930, wurde im Bereich der Mittelspannungs- und Niederspannungsebene bereits die Tonfrequenz-Rundsteuertechnik (TRT) zur Nachrichtenübertragung eingesetzt [46], [161], [174].

Werden vorhandene elektrische Leitungen im Niederspannungsnetz zum Aufbau eines lokalen Netzwerkes für die Datenübertragung verwendet, spricht man von der sogenannten Powerline Communication (PLC) oder kurz Powerline. Die erstmalige Verwendung des Begriffes Powerline Communication findet sich in den 1970er-Jahren in den USA [80]. Hier wurden erste Versuche unternommen, eine bidirektionale Datenübertragung über das Niederspannungsnetz aufzubauen. In Deutschland finden sich erste Anfänge im Jahre 1980 mit dem sogenannten „Babyphone“ in den privaten Haushalten. Mittlerweile kennt man PLC auch als „Internet aus der Steckdose“ [47]. Hier dient das hausinterne Stromnetz als schnelle Kommunikationsleitung, um unterschiedliche Teilnehmer wie PCs, Drucker, Tablets usw. miteinander zu vernetzen.

In dieser Arbeit wird der Ansatz der Datenkommunikation über das Stromnetz bzw. über ein induktives kontaktloses Energieübertragungssystem aufgegriffen und umgesetzt. Die vorliegende Arbeit beschäftigt sich ferner mit der Erstellung eines Konzeptes für die Einbindung eines intelligenten Aktors in den Bereich der induktiven kontaktlosen Energieübertragung. Neben der beschriebenen möglichen Realisierung der Datenanbindung über PLC stellt sich die Frage, wie der konkrete Aktor aufgebaut sein kann. Im nächsten Abschnitt wird zuerst auf die Begrifflichkeit eines mechatronischen Systems und auf die verschiedenen Arten von Aktoren eingegangen. Im Abschnitt 1.3 werden die Ziele und Aufgaben beschrieben, wie ein intelligenter Aktor aufgebaut werden kann.

\subsubsection{Aktoren und mechatronische Systeme}

Im Rahmen dieser Arbeit wird der Entwurf und die Entwicklung eines intelligenten Aktors (engl.: Smart Actuator) vorgestellt. Der intelligente Aktor ist vor allem gekennzeichnet durch seine Kommunikationsfähigkeit und die Möglichkeit, komplexe Aufgaben selbstständig zu bearbeiten. Dazu gehören z.B. die Abarbeitung komplexer Regelungsalgorithmen oder die Auswertung von Diagnosefunktionen.

In der Arbeit soll auch untersucht werden, wie ein solcher intelligenter Aktor in den Bereich der induktiven berührungslosen Energieübertragung eingebunden werden kann. Im folgenden Abschnitt wird zuerst auf die unterschiedlichen Arten und Prinzipien von Aktoren innerhalb mechatronischer Systeme eingegangen. 
Der Verein Deutscher Ingenieure definiert die Mechatronik in seiner Richtlinie 2206 [222] als „das synergetische Zusammenwirken der Fachdisziplinen Maschinenbau, Elektrotechnik und Informationstechnik beim Entwurf und der Herstellung industrieller Erzeugnisse sowie bei der Prozessgestaltung“. Durch die Integration der einzelnen mechanischen, elektronischen und zugehörigen informationsverarbeitenden Elemente können durch konstruktive Verschmelzung erweiterte Funktionen und synergetische Effekte erzielt werden [24], [43], [84].

Die mechanischen Komponenten eines mechatronischen Systems betreffen die Bereiche der Maschine bzw. der Maschinenelemente selbst. Elektronische Systeme beinhalten die Bereiche der Mikro- und Leistungselektronik, Messtechnik und Aktorik. Die informationsverarbeitenden Elemente enthalten die Themen der Systemtheorie und der Software-Realisierung sowie die Umsetzung steuerungs- und regelungstechnischer Aufgaben - auch die mögliche Einbindung von Künstlicher Intelligenz [97], [107], [232]. Die Grundstruktur eines mechatronischen Systems setzt sich im Wesentlichen aus folgenden Teilkomponenten zusammen [43]:

- Mechatronische Bauelemente

- Sensoren zur Erfassung funktionsrelevanter Messgrößen

- Prozessoren mit Algorithmen zur Signalverarbeitung

- Aktoren mit Stellgrößen für Regelung und Steuerung.

In Abbildung 1.3 ist der Aufbau eines mechatronischen Systems dargestellt. Das mechanische System wird durch Sensoren, Aktoren und Mikrorechner zur Realisierung intelligenter oder zumindest teilintelligenter Produkte erweitert. Dabei ist die mechanische Grundstruktur des mechatronischen Systems gekennzeichnet durch Eingangsgrößen und Ausgangsgrößen. Die funktionsrelevanten Messgrößen werden von den Sensoren erfasst, in elektrische Signale umgewandelt und dem Prozessor zugeführt. Nach der Verarbeitung der Prozessdaten durch den Prozessor werden entsprechende Stellgrößen an den Aktor zur Optimierung des Systems weitergegeben.

\section{Aktoren}

Aktoren, auch als Aktuatoren bezeichnet, haben in der Regel eine elektrische Eingangsgröße und eine mechanische Ausgangsgröße. Dabei kann die Ausgangsgröße z.B. ein Weg, eine Geschwindigkeit oder eine Kraft sein. Abbildung 1.4 zeigt die Funktion eines allgemeinen mechatronischen Aktors. Die Eingangsgröße für den Aktor bildet die Vorgabe der Stellgröße, die von einer Steuerung oder Regelung vorgegeben wird. Die Hilfsenergie kann dabei elektrisch, hydraulisch oder pneumatischer Natur sein und der Anwendung zur Verfügung gestellt werden. 


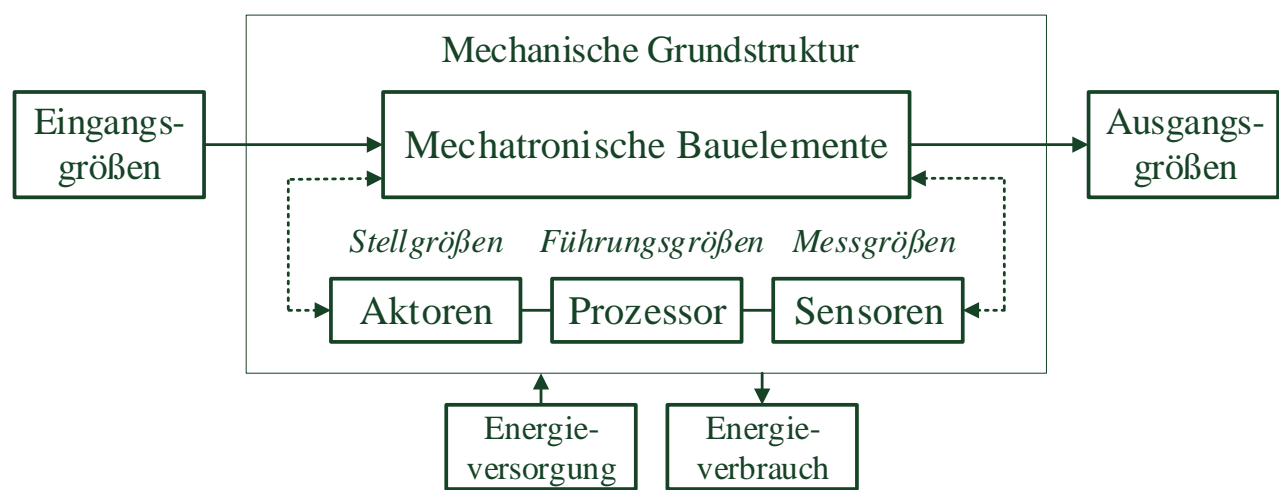

Abbildung 1.3.: Aufbau eines mechatronischen Systems [43].

Der Aktor, der dann als Stelleinrichtung fungiert, beeinflusst durch die Stellenergie den technischen Prozess. Da Aktoren in vielen technischen Bereichen für unterschiedliche Applikationen zum Einsatz kommen, zeichnen sie sich in der Praxis durch vielfältige Bauformen aus.

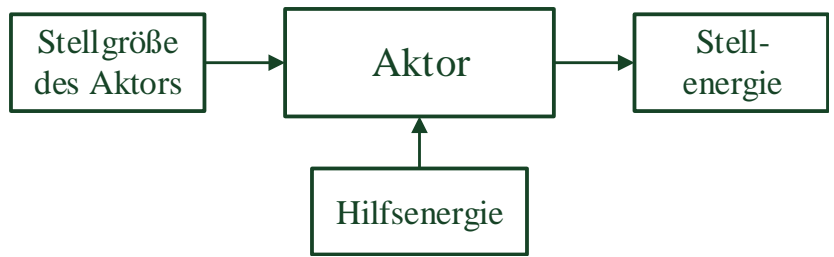

Abbildung 1.4.: Darstellung eines Aktors mit Ein- und Ausgangsgröße [43].

In [43] werden verschiedene Aktortypen nach der für die Aktor-Funktion benötigten Hilfsenergie eingeteilt. Es finden sich aber in der Literatur auch zahlreiche Beispiele für eine genauere Einteilung durch die Unterscheidung weiterer Prinzipien [87], [218]. Folgende Aktortypen werden in [43] unterschieden:

- Elektromechanische Aktoren

- Piezoelektrische Aktoren

- Fluidtechnische Aktoren

- Thermomechanische Aktoren.

\section{Elektromechanische Aktoren}

Elektromechanische Aktoren sind weit verbreitet und zeichnen sich durch eine breite Variantenvielfalt aus. Besonders motorische Stellantriebe kommen für unterschiedlichste Stellaufgaben zur Anwendung. Die Abbildung 1.5 zeigt ein Beispiel für einen einfachen Aufbau eines elektromechanischen Aktors. 


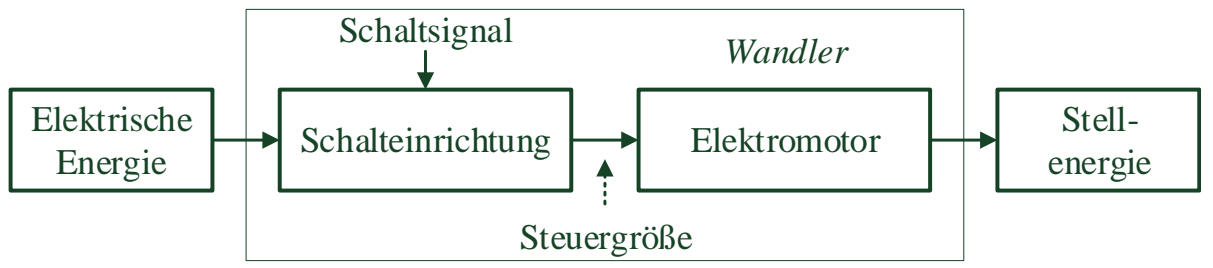

Abbildung 1.5.: Aufbau eines einfachen elektromechanischen Aktors [43].

Die Stellgröße wird durch eine Schalteinrichtung, z.B. durch ein Relais, Schütz oder einer elektronischen Schaltung, in eine Steuergröße überführt. Die Steuergröße bildet die Eingangsgröße des Stellantriebes, z.B. für einen Elektromotor oder Elektromagneten. Der Stellantrieb wandelt die Hilfsenergie, gesteuert durch die Steuergröße, in die für die Funktion des Aktors benötigte Stellenergie um.

Am Ausgang des Wandlers wird die Stellenergie bzw. ein Stellweg in Form einer Translations- oder Rotationsbewegung zur Verfügung gestellt. Wenn erforderlich wird am Ausgang des Wandlers ein Getriebe nachgeschaltet. Die Stellenergie wird durch das Getriebe entsprechend den technischen Anforderungen in Kraft, Drehmoment bzw. Geschwindigkeit überführt. Elektromechanische Aktoren zeigen, gerade bei motorischen Stellantrieben, bei hoher Dynamik gute Regeleigenschaften. Neben einem guten Stellverhalten und hoher Positioniergenauigkeit lassen sich flexible Antriebskonzepte realisieren [18], [88]. Nachteilig wirken sich u.a. die eingeschränkte Leistungsdichte und der eingeschränkte thermische Betriebsbereich aus [84].

\section{Piezoelektrische Aktoren}

Die Abbildung 1.6 zeigt den Aufbau des piezoelektrischen Aktors. Die Eingangsgröße des Aktors bildet die elektrische Energie, die Ausgangsgröße des Aktors erzeugt mechanische Stellarbeit.

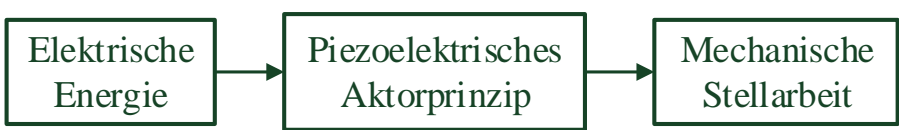

Abbildung 1.6.: Aufbau eines piezoelektrischen Aktors [43].

Werden bestimmte Kristalle, wie z.B. Quarze, mechanisch verformt, führt die Verformung zu Verschiebungen der Ionen in der Kristallstruktur (vgl. Abschnitt B.1). Die Polarisierung der Ionen erzeugt eine Spannung im Kristall, die durch Elektroden an der Kristalloberfläche gemessen werden kann. Der physikalische Zusammenhang zwischen der mechanischen Belastung des Kristalls und der elektrischen Ladungsverschiebung wird als direkter piezoelektrischer Effekt [74] bezeichnet und findet Anwendung für die Druck- und Kraftwandlung. Der Effekt ist auch umkehrbar. Durch Anlegung einer elektrischen Spannung an den Elektroden des Kristalls dehnt sich 
der Kristall aus. In der Aktorik wird der inverse Piezoeffekt [74] zur Erzeugung von Bewegungen durch Anlegen elektrischer Spannungen an Piezokristallen genutzt.

Eine Anwendung finden solche Aktoren z.B. als Piezo-Aktoren im Tintenstrahldrucker. Die elektrisch ansteuerbaren Piezo-Schwingmembranen befinden sich auf der Druckkammer zwischen Tintenkanal und Auslassdüse. Die Piezoschwinger erzeugen durch Spannungswechsel Saug- und Druckimpulse für den Tintentransport und bewirken dadurch eine Tröpfchenbildung bzw. einen Tröpfchenflug zum Papier. Piezoelektrische Aktoren werden für Stellwege im Nanometer-Bereich angeboten. Die Positioniergenauigkeit beträgt dabei $<1 \mathrm{~nm}$. Stellkräfte von $1 \ldots 5000 N$ bei hoher Steifigkeit sind möglich [43], [84].

\section{Fluidtechnische Aktoren}

Fluidtechnische Aktoren (pneumatisch oder hydraulisch) sind durch einen robusten Aufbau, eine hohe Leistungsdichte und große Stellkräfte und Stellbereiche gekennzeichnet [86], [218]. Die Eingangsgröße des fluidtechnischen Aktors in Abbildung 1.7 bildet ein Druck/Volumenstrom bzw. Strömungsenergie. Vorteilhaft ist die direkte und einfache Umsetzung einer Linearbewegung über Stellzylinder. Gleichzeitig findet kein Energieumsatz im stationären Betrieb statt.

Nachteilig ist, dass der Gesamtwirkungsgrad des Systems meist schlechter ist als bei elektrischen Antrieben. Oft ist es auch nachteilig, dass zusätzliche Hilfsenergieerzeuger notwendig sind. Der Aufbau erfordert dabei komplexe Systemstrukturen und eine anspruchsvollere Automatisierungslösung. Dabei ist die Positionsgenauigkeit eingeschränkt [84].

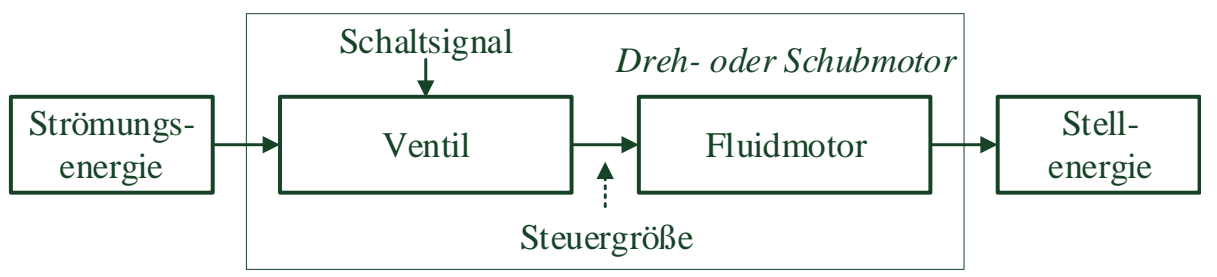

Abbildung 1.7.: Aufbau eines fluidtechnischen Aktors [43].

\section{Thermomechanischer Aktor}

Die Eingangsgröße des thermomechanischen Aktors ist die thermische Energie (Abbildung 1.8). Am Ausgang des Aktors wird mechanische Stellarbeit zur Verfügung gestellt. Der Stellantrieb des Aktors wird durch die Zuführung thermischer Energie bzw. über die Temperatur gesteuert. In der Regel benötigt der robuste Aktor selbst keine eigene elektrische Spannungsversorgung. Die Aktoren sind geeignet als Schaltelemente, z.B. als Bimetallschalter in Bügeleisen oder für die Verwendung in Leitungsschutzschalter [18], [43], [231]. 


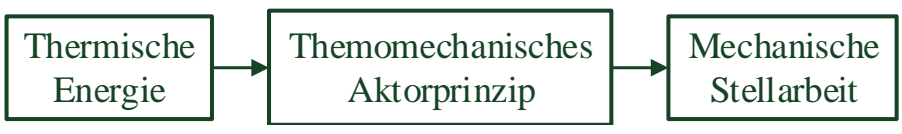

Abbildung 1.8.: Aufbau eines thermomechanischen Aktors [43].

Die meisten der vorkommenden Typen der thermomechanischen Aktoren sind Dehnstoff-Aktoren, sie werden für hohe Stellkräfte eingesetzt. Dehnstoff-Aktoren nutzen die große Volumen-Temperatur-Abhängigkeit von festen und flüssigen Stoffen mit hohem Wärmeausdehnungskoeffizient. Durch Zuführung thermischer Energie und auftretender Volumenzunahme wird eine Aktor-Bewegung umgesetzt. Weitere Anwendungsformen für thermomechanische Aktoren finden sich bei Formgedächtnisaktoren. Ausgangspunkt ist hier die Verwendung sogenannter Formgedächtnislegierungen, mit denen sehr große Kräfte übertragen werden können [43].

\section{Intelligente Aktoren}

Die Abbildung 1.3 zeigt den Aufbau eines mechatronischen Systems mit der hardwareseitigen Integration der Komponenten. Sensoren, Aktoren und Mikroprozessor sind in den mechanischen Prozess integriert. Wird der Mikroprozessor mit dem Aktor, Prozess oder Sensor integriert, wird die entstandene Verbindung als Embedded System [28], [85], [130] bezeichnet.

Mikroprozessor und integrierte Sensoren entwickeln sich zu intelligenten Sensoren (engl.: Smart Sensors) [138]. Mikroprozessor und integrierte Aktoren zu intelligenten Aktoren. Durch die Integration der elektronischen Komponenten müssen zusätzliche Anforderungen wie die Entwärmung der Bauteile oder andere Einflüsse, wie z.B. Verschmutzung, Erschütterungs- und Stoßbelastungen während des Betriebes, berücksichtigt werden [84]. Werden einfache mechatronischen Systeme durch eine bessere Informationsgewinnung und höhere Informationsverarbeitung erweitert, entwickeln sich daraus intelligente Geräte und intelligente Maschinen [178].

Der Beginn des Zeitalters der intelligenten oder smarten Aktorik nimmt ihren Anfang in den 1990er-Jahren durch die immer stärkere Einbindung und Integration der Mikroprozessoren in die Aktuatoren. Durch das kompaktere Systemdesign konnten mehr Funktionen in den Aktuator integriert werden, komplexere Regelstrategien ließen sich einbinden, gleichzeitig vereinfachte sich der Installationsaufwand des Aktors bzw. der mechatronischen Einheit. Auch die Möglichkeit eines modularen Aufbaus wurde durch die neuen Baugruppen gegeben. Durch die integrierte Elektronik konnten die Aktuatoren auch immer besser in Netzwerke und Feldbusse eingebunden werden.

Der Begriff des intelligenten Aktors findet sich im technischen Umfeld in vielen Bereichen. Intelligente Aktoren sind bekannt aus dem Bereich der Gebäudetechnik, wo einfache zeitgesteuerte Funktionen autonom ausführt werden. Einfache Beispiele 
sind z.B. Treppenhauslichtschalter oder das Steuern von Bewegungsmeldern. Auch Heizungsthermostate, die autonom arbeiten, mit Zeitplan und Anwesenheitserkennung oder die über das Internet angesteuert werden können, werden als smarte oder intelligente Aktoren angeboten [1].

In [172] werden intelligente Aktoren im Bereich der Arbeitsumgebung zur Steigerung des Wohlbefindens und der Produktivität betrachtet. In den letzten Jahren finden sich smarte Aktoren auch verstärkt im Bereich des Automobilbaus. Kleine, smarte Aktoren im zweistelligen Watt-Bereich fungieren als kompakte Stellantriebe, Ventile und für andere Anwendungen [119].

\subsubsection{Intelligente Aktoren als Systemkomponenten}

Die immer stärkeren Vernetzungen und Verzahnungen bei mechatronischen Systemen führen dazu, dass auch die Aktoren selbst immer stärker als Systemkomponenten in das Gesamtsystem innerhalb der mechatronischen Einheit eingebunden werden. Die Merkmale, die eine Integration in das Gesamtsystem ermöglichen, sind [84]:

- Art der Hilfsenergie

- Eingangs- und Ausgangsverhalten

- Schnittstellen

- Bauliche Integration mit dem Prozess

- Ausgeführte Funktionen, Grad der Intelligenz

- Maßnahmen zur Erhöhung der Zuverlässigkeit.

Wird die Regelung eines Aktors durch integrierte Mikroprozessoren und intelligente Softwarestrukturen erweitert, können die Stelleigenschaften des Aktors deutlich verbessert werden. Durch die zusätzlichen ausgestatteten Funktionen und Eigenschaften entwickelt sich das Stellglied zum intelligenten Aktor und fungiert als intelligente Systemkomponente innerhalb eines mechatronischen Systems.

Mechatronische Systeme entstehen durch die Verschmelzung mechanisch-elektronischer Systeme bzw. durch eine geeignete Kombination von Mechanik, Elektronik und Informationsverarbeitung. Dabei beeinflussen sich die Bereiche gegenseitig, wobei durch die Verwendung und Verfügbarkeit einer immer leistungsfähigeren und kompakteren Elektronik auch viele Funktionen, die früher mechanisch realisiert wurden, jetzt elektronisch umgesetzt werden.

Wo z.B. früher Maschinen bei Anforderung eines mechanischen Gleichlaufs über eine starre Welle miteinander gekoppelt bzw. synchronisiert wurden, werden in heutigen 
Anwendungen mechatronische Einheiten mit integrierter Elektronik eingesetzt. Über eine elektronische Kopplung, z.B. via Feldbus-Kommunikation, kann die Möglichkeit eines elektronischen Maschinengleichlaufs einfach realisiert werden.

Modellbasierte Regelungen und Steuerungen für solche Geräte können z. B. so aufgebaut sein, dass sie sich an das meist nichtlineare Verhalten der Regelstrecke mittels intelligenter Regelalgorithmen anpassen können. Abhängig vom Arbeitspunkt der Maschine werden die Parameter der Regelung „eingelernt“. Bei veränderten Randbedingungen oder Umweltbedingungen adaptieren sich solche selbstlernende Systeme selbstständig an die neuen Anforderungen und Bedürfnisse [57], [116], [129].

Gleichzeitig wird der Prozess permanent überwacht und auf ein mögliches Fehlverhalten analysiert. Fehler der Maschine oder Anlage werden diagnostiziert und es wird auf eine evtl. notwendige Wartung im Sinne einer Prospektiven Instandhaltungsstrategie (engl.: Predictive Maintenance) in der nahen Zukunft verwiesen.

Neben den quantitativen Entwurfs- und Optimierungsmethoden mit analytischen Prozessmodellen kommen für Prozesse, die sich einer genaueren analytischen Beschreibung entziehen, Methoden des Soft-Computing zur Anwendung. Zu den Kerngebieten des Soft-Computings zählen die Teilgebiete der Künstlichen Intelligenz wie Fuzzylogik und Fuzzy-Mengen, [19], [102], Künstliche Neuronale Netze [103], [112], [176] und die Gebiete der Evolutionären Algorithmen [116], [180], [228].

Während die Evolutionären Algorithmen bei Optimierungsverfahren zum Einsatz kommen, liegt der Schwerpunkt der Fuzzylogik im regelungstechnischen Bereich. Künstliche Neuronale Netze finden in der Regel Anwendung im Bereich der Klassifikation und Mustererkennung [84]. Verwendung finden solche intelligente Systeme u.a. für die Steuerung selbstnavigierender Fahrzeuge oder mobiler Roboter [15], [142], [216].

Zusammenfassend zeichnen sich intelligente Aktoren durch folgende Eigenschaften und Funktionen aus [43], [84]:

- Beinhalten eigene Steuerungs- und Regelungsintelligenz, wie z.B. modellgestützte, nichtlineare Regelungen mit adaptivem Verhalten

- Werten selbstständig für sie relevante Sensorsignale aus und führen selbst Berechnungen für den Prozesseingriff durch

- Erkennen Fehler (auch prädiktiv) mit modellgestützten Methoden wie z.B. durch die Verwendung von Paritätsmethoden oder Einbindung von Zustandsbeobachtern

- Verfügen über eine selbstständige Fehlerdiagnose mit Angaben über Fehlerarten und Wartungshinweisen 
- Besitzen die Fähigkeit, energie- und verschleißoptimale Stellstrategien umzusetzen bzw. energetisch und materialschonend optimal auf den Prozess einzuwirken.

\section{Intelligenter Servoantrieb}

Ein bekannter Vertreter des intelligenten Aktors ist der dezentrale Servoantrieb. Die Abbildung 1.9 zeigt den prinzipiellen Aufbau. Der Aktor zeichnet sich durch seine dezentrale Intelligenz und seinen kompakten Aufbau aus.

Ein vorhandener Motor- oder Streckengeber wird direkt durch die interne Elektronik ausgewertet. Neben dem geschlossenen Regelverfahren mit Motorgeber ist es intelligenten Aktoren technisch möglich, einen geberlosen geregelten Betrieb, unter Berücksichtigung der Applikationsanforderungen, zu realisieren. So ist es auch möglich, Synchronmotoren in der Betriebsart feldorientierte Regelung (FOC, engl.: Field Oriented Control ohne Rotorlagegeber zu betreiben [150], [154].

Auf der Eingangsseite in Abbildung 1.9 wird der dezentrale Aktor in der Regel über die Busschnittstelle bzw. die integrierte Elektronik mittels Feldbus an eine übergeordnete Steuerung oder einen Leitrechner angebunden. Neben der eingangsseitigen Energieversorgung sind mittlerweile viele Umrichter im Bereich der Funktionalen Sicherheit mit zusätzlichen Sicherheitsfunktionen wie STO (engl.: Safe Torque Off) oder einer sicheren Kommunikation ausgestattet. Bei der Sicherheitsfunktion STO wird nach dem Wegschalten des STO-Freigabesignals die Energieversorgung der Leistungshalbleiter hardwareseitig sofort unterbrochen. Der Antrieb kann nach der Abschaltung kein Drehmoment mehr aufbauen. Eine zusätzliche aufwendige äußere Beschaltung, wie sie früher bei älteren Geräten für eine Notabschaltung notwendig war, kann eingespart werden.

Doch die heutigen Anforderungen an einen intelligenten Aktor sind weit mehr. Neben einer vertikalen Informationsanbindung an den Leitrechner oder die übergeordnete SPS wird von heutigen intelligenten Aktoren gefordert, dass sie auch horizontale bzw. Quersegmente und unterlagerte Segmente, bestehend aus Sensoren und Aktoren und anderen Antrieben oder mechatronischen Systemen ansprechen und auswerten können. Zusammen mit den eigenen zur Verfügung stehenden Daten und den zusätzlichen peripheren Informationen, die alle lokal verarbeitet werden, ist es solchen Architekturen möglich, selbst zu entscheiden, wie Abläufe generiert werden und wie die Bewegungen innerhalb des Prozesses ablaufen.

Durch die lokale Datenaufbereitung und Datenverarbeitung, in der Abbildung 1.9 durch den Optimierungsblock dargestellt, werden übergeordnete Steuerungsprogramme im Leitrechner einfacher und weniger komplex, gleichzeitig verringert sich die Feldbusauslastung. Neben den Koordinierungs- und Kommunikationsaufgaben zum Feldbussystem werden vom Mikrocomputer die regelungstechnischen Aufgaben und 


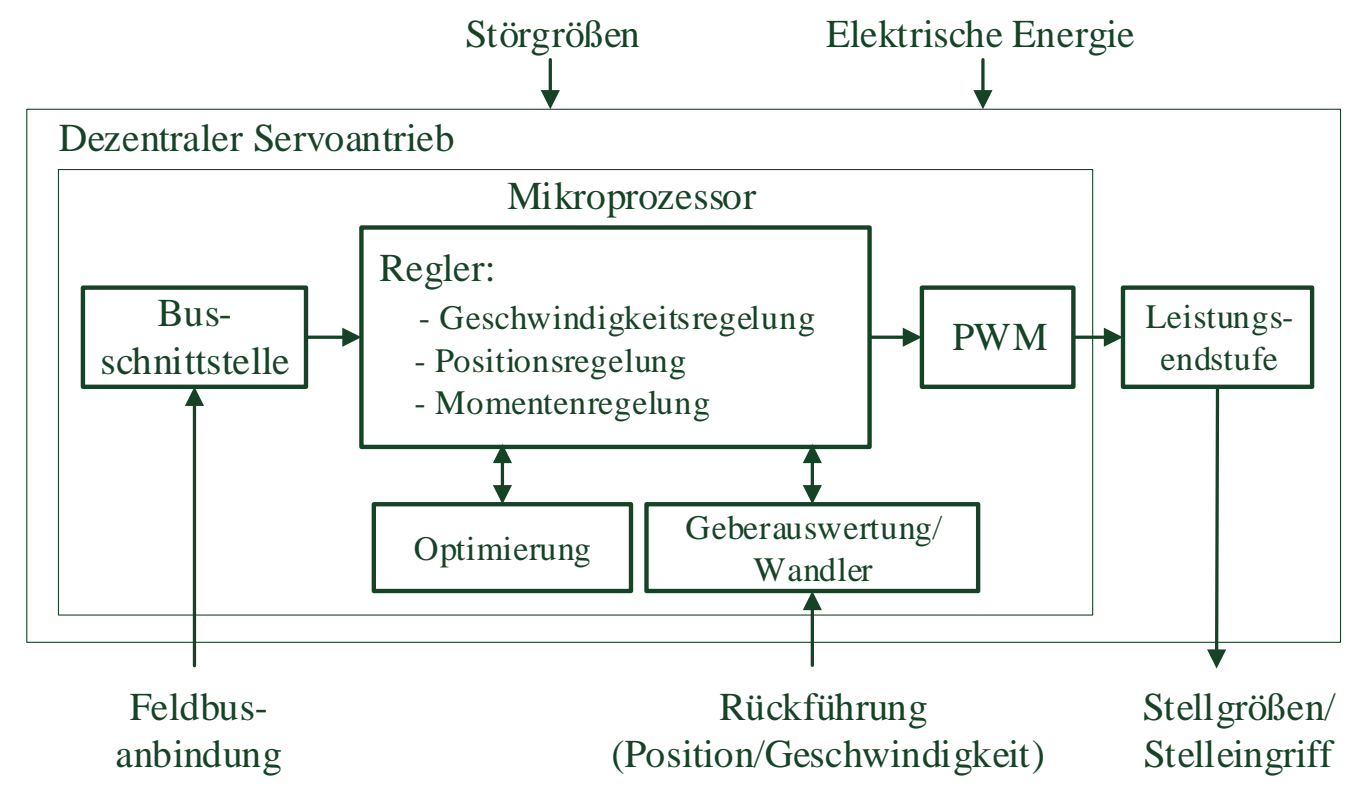

Abbildung 1.9.: Aufbau eines intelligenten Aktors am Beispiel eines Servoantriebes [43], [84].

Diagnosefunktionen lokal verarbeitet. Ausgangsseitig steuert der Umrichter über die Pulsweitenmodulation (PWM) und die Leistungsendstufe die Stellgröße bzw. den Motor.

Solche flexible Komponenten haben den Anspruch, sich autark vernetzt in die digitale Welt oder Industrie 4.0 innerhalb einer flexiblen Steuerungsarchitektur einzubinden. Dabei werden vitale Daten des Antriebes wie Leistungsverbrauch, Einschaltdauer, Auslastung usw. gesammelt, analysiert und ausgewertet. Durch Auswertung der Informationen können Lastprofile der einzelnen Maschinen und Anlagenteile berechnet und der Materialfluss in der Anlage optimiert werden. Mittels der Informationen kann auch die Antriebsauslegung auf den konkreten Anwendungsfall angepasst werden, auch hinsichtlich einer energetischen und einer materialschonenden Betriebsweise des Antriebs. Bei der Betrachtung der Definition eines intelligenten Aktors mit zusätzlichen funktionellen Möglichkeiten und vernetzter Intelligenz ist der Übergang des intelligenten Aktors zum mechatronischen System immer mehr gleitend.

\subsection{Ziele und Aufgaben}

Das Ziel der vorliegenden Arbeit besteht darin, ein neues Konzept für einen dezentralen intelligenten Aktor zu entwickeln, der kontaktlos über ein induktives Energieübertragungssystem mit Energie versorgt wird. Neben der Energieversorgung soll auch die 
Datenkommunikation von der übergeordneten Steuerung zum Aktor kontaktlos realisiert werden. Dazu wird der intelligente Aktor hinsichtlich der erforderlichen Hardund Softwarefunktionalität aufgebaut. Auf der regelungstechischen Seite wird untersucht, inwieweit ein modellprädiktives Regelungsverfahren im Entwurf des Aktors eingebunden werden kann.

Die Kommunikationsübertragung zwischen der übergeordneten Steuerung und dem dezentralen Aktor baut auf dem Prinzip der Powerline Communication (PLC) auf. PLC nutzt, wie bereits in Abschnitt 1.2 ausgeführt, den vorhandenen Energiekanal, hier den Linienleiter, auch als Kommunikationskanal. Das Kommunikationssignal wird dem Energieverteilungsnetz aufmoduliert und steht an jeder Stelle des Stromnetzes zur Verfügung. Dadurch, dass die Energieleitungen auch gleichzeitig als Kommunikationsleitungen Verwendung finden, werden Material- und Installationskosten eingespart.

Gleichzeitig ist der Linienleiter, als Datenkanal betrachtet, bandbegrenzt und stark störbehaftet. Das Übertragungsverfahren muss deshalb besonders robust sein. Bei einer großen Anzahl von Kommunikations-Teilnehmern und gleichzeitigem hohen Anspruch an Gleichzeitigkeit und Synchronität zwischen den Teilnehmern (vgl. Abschnitt 4.1) stellt die relativ geringe Datenübertragungsrate den „Flaschenhals" für viele Anwendungen dar.

Aus den genannten Gründen wird in der Arbeit untersucht, inwieweit über ein Zeitstempelverfahren bewegliche elektrische Verbraucher miteinander synchronisiert werden können. Das Zeitstempelverfahren beruht auf dem Prinzip von lokal implementierten Uhren in jedem Teilnehmer, wobei über den Zeitstempel die steuerungs- und regelungstechnischen Abläufe koordiniert und gleichzeitig das Datenaufkommen auf dem Übertragungsmedium, hier dem Linienleiter, reduziert werden soll. Die Implementierung des Verfahrens setzt dabei hochgenaue Uhren in jedem Teilnehmer voraus.

Für die Umsetzung des neuen Konzeptes sind im wesentlichen folgende Punkte zu untersuchen und zu erarbeiten:

- Aufbau eines induktiven kontaktlosen Energieübertragungssystems und Analyse des Übertragungsverhaltens des Datenkanals, der über die induktive kontaktlose Energieversorgung zu realisieren ist

- Untersuchung, wie eine Datenkommunikation und eine Datensynchronisation über das induktive kontaktlose Energieübertragungssystem ermöglicht werden kann

- Realisierung einer neuen Hardware- und Softwareplattform für einen intelligenten kompakten Aktor im Bereich der induktiven kontaktlosen Energieübertragung 
- Entwicklung eines modellprädiktiven Regelungskonzeptes für einen intelligenten Aktor im Bereich der beweglichen elektrischen Verbraucher.

In Kapitel 2 der Arbeit wird im ersten Schritt ein neues Konzept vorgestellt, wie ein intelligenter Aktor im Umfeld der induktiven kontaktlosen Energieübertragung aufgebaut werden kann. Die Struktur des neuen Konzeptes und die zu entwickelnden funktionellen Komponenten werden definiert und dargestellt. Es wird gezeigt, wie die unterschiedlichen Funktionsblöcke für die Kommunikation, Datensynchronisierung und Regelung in das Gesamtkonzept des intelligenten Aktors eingebunden werden.

Im darauf folgenden Kapitel 3 wird der Aufbau eines induktiven kontaktlosen Energieübertragungssystems beschrieben. Das Ziel dieses Versuchsaufbaues ist u.a. auch zu zeigen, wie ein solches System für eine rotative Prozessbewegung konstruktiv umgesetzt werden kann. Der Versuchsaufbau ist eine wichtige Voraussetzung für die nachfolgenden Untersuchungen des Datenübertragungsverhaltens über den induktiven kontaktlosen Energieübertragungskanal in Kapitel 4. Für die Bestimmung des Datenübertragungsverhaltens werden dafür im Abschnitt 3.5 die Eigenschaften und die Störeinflüsse des Datenübertragungskanals analysiert und bewertet.

In Kapitel 4 wird untersucht, inwieweit eine Datenkommunikation zwischen den Teilnehmern über den induktiven kontaktlosen Energieübertragungskanal möglich ist und welches Nachrichtenübertragungsverfahren und Modulationsverfahren sich dafür eignet. Es wird gezeigt, wie eine Synchronisation zwischen den Teilnehmern über den Linienleiter realisiert wird und welche Synchronisationsgenauigkeit bei der Synchronisation zwischen den Teilnehmern an dem konkreten Versuchsaufbau erreichbar ist.

Im weiteren Verlauf wird in Abschnitt 4.5 auf die Realisierung hochgenauer Uhren auf Basis unterschiedlich genauer Oszillatoren eingegangen. Untersucht wird, welche Ganggenauigkeiten bei verschiedenen Oszillatorklassen erreicht werden und welche Eigenschaften bzw. Merkmale wichtig für die verwendeten Komponenten sind. In einem praktischen Versuchsaufbau wird die Ganggenauigkeit von Oszillatoren unterschiedlicher Oszillatorklassen durch die Zwei-Oszillatoren-Methode qualitativ bewertet. Das Ziel der Untersuchung ist zu zeigen, inwieweit sich durch den Einsatz von höherwertigen Oszillatoren die Taktfrequenz für die Synchronisierungsvorgänge bei der Übertragung von Daten zwischen den einzelnen Kommunikationsteilnehmern reduzieren lässt.

Nach der Untersuchung der Synchronisationsgenauigkeit zwischen den Teilnehmern stellt sich in Kapitel 5 die Frage, wie es möglich ist, einen konkreten motorischen Aktor in das vorgestellte Gesamtkonzept in Kapitel 2 einzubinden. Es wird untersucht, wie ein kompakter intelligenter motorischer Aktor alternativ aufgebaut werden kann, wobei die funktionellen Komponenten des Kommunikationsmoduls aus Kapitel 4 mit 
den funktionellen Komponenten zur Ansteuerung des Aktors auf einer gemeinsamen Systemplattform zusammengefasst werden.

Dabei wird gezeigt, wie die FOC für einen solchen Aktor durch eine abbildbare Hardware- und Softwareumgebung realisiert wird und wie u.a. die wichtige Phasenstrommessung des motorischen Aktors mit modernen Strommessverfahren integriert werden kann. Es stellt sich auch die Frage, wie der Aktor durch Auswahl und Zuordnung unterschiedlicher Funktionsmodule strukturell aufbaubar ist.

In Kapitel 6 werden unterschiedliche regelungstechnische Konzepte betrachtet, wie ein intelligenter Aktor im Konzept für bewegliche elektrische Verbraucher eingebunden werden kann. In einer Simulation wird die Leistungsfähigkeit einer modelprädiktiven Regelung im Vergleich zu einer klassischen PID-Regelung untersucht. In der durchgeführten Simulation werden die Vorteile des prädiktiven Verhaltens einer solchen Regelung im System der hochgenauen Uhren auf Basis von Zeitstempeln aufgezeigt.

Im letzten Kapitel 7 erfolgt eine Zusammenfassung über die in den einzelnen Kapiteln erarbeiteten Lösungen und Ergebnisse. 


\section{Neues Konzept für die Optimierung intelligenter Aktoren}

Im Bereich der mobilen Intralogistik und angrenzenden Gebieten des Maschinenbaus lassen sich viele logistische, montage- und produktionstechnische Prozesse durch den Einsatz der induktiven kontaktlosen Energieübertragung flexibel und effizient abbilden.

Dabei hat die induktive kontaktlose Energieversorgung von mobilen bzw. beweglichen elektrischen Verbrauchern vor allem dort ihre Vorteile und Einsatzfelder, wo Maschinen- und Anlagenkomponenten Kreis- bzw. Rotationsbewegungen durchlaufen oder wo lange Verfahrstrecken in verschiedene Richtungen realisiert werden müssen. Vorteile ergeben sich in Bereichen spurgeführter Anwendungen, wie z.B. einer Elektrohängebahn oder einer Schubskidanlage [99], [118].

In Bereichen von teilweisen spurgeführten oder freifahrenden Anwendungen, wie z.B. bei Fahrerlosen Transportsystemen oder mobilen Assistenzsystemen (vgl. Abbildung 2.1), die auch immer stärker im Bereich des Umfeldes der Industrie 4.0 eingesetzt werden, ist die Verwendung einer kontaktlosen Energieversorgung ebenfalls in gleicher Weise sinnvoll. Die wesentlichen Systemvorteile gegenüber einer klassischen Energieübertragung (z.B. bei der Verwendung von Schleifringen), sind zum einen die verschleißfreie Energieübertragung selbst, dass heißt keine Wartung oder Abnutzung der Komponenten und die Robustheit gegenüber Einflüssen wie Verschmutzung, Feuchtigkeit und Temperatur [39], [153], [197].

Solche Anlagen oder Maschinen besitzen in der Regel einen zentralen Energie-Einspeiseknoten, von wo aus die mobilen Endgeräte versorgt werden. Eine stationäre Einheit versorgt dabei meist mehrere Verbraucher. Reicht eine stationäre Einheit bedingt durch die Anzahl der mobilen Endgeräte nicht aus, können segmentartige Strukturen aufgebaut werden. Für den Kommunikationsaufbau zwischen den Teilnehmern bzw. für den Aufbau eines Bussystems macht es deshalb Sinn, den zentralen Energie-Einspeiseknoten auch als zentralen Punkt für den Kommunikationsknoten nach außen zur externen Steuerung oder zum zentralen Server zu wählen. Neben der Energieversorgung sollte natürlich auch die Datenübertragung bzw. die Datenkommunikation zwischen den Teilnehmern kontaktlos erfolgen. 
Abbildung 2.1.: Mobiles Assistenzsystem für die Getriebemontage [205].

Während die induktive kontaktlose Energieübertragung bereits am Markt von verschiedenen Herstellern für vielfältige Applikationen angeboten wird und verfügbar ist [39], [153], [197], werden für die Datenübertragung über den induktiven kontaktlosen Energiekanal kaum technische Lösungen angeboten. Auch eine effektiv geeignete datentechnische Synchronisierung der Teilnehmer zur zentralen Steuerung oder zwischen den Teilnehmern ist als Produkt am Markt nicht verfügbar.

In der weiterführenden Betrachtung stellt sich daher die Frage, wie ein mobiler bzw. beweglicher elektrischer Verbraucher, der über das induktive kontaktlose Energieübertragungssystem eingebunden ist und mit Energie versorgt wird, hardware- und softwaretechnisch hinsichtlich möglicher Kommunikations- und Steuerungsaufgaben zu realisieren ist. Gleichzeitig sollte es möglich sein, einen solchen elektrischen Verbraucher bzw. kompakten intelligenten Aktor flexibel in einem solchen technischen Umfeld einzubinden.

Im nachfolgenden Abschnitt wird ein neues Konzept vorgestellt, wie ein kompakter intelligenter Aktor mit induktiver kontaktloser Energieübertragung im Bereich der beweglichen elektrischen Verbraucher aufgebaut ist. Zuerst wird die Grundstruktur des neuen Konzeptes eines intelligenten Aktors vorgestellt. 


\subsection{Grundstruktur des neuen Konzeptes}

Die Abbildung 2.2 zeigt übersichtlich einen ersten vereinfachten Entwurf des zu entwickelnden intelligenten aber auch kompakten Aktors im Bereich der beweglichen elektrischen Verbraucher. Der Aktor setzt sich in der Abbildung 2.2 aus unterschiedlichen Funktionsblöcken bzw. funktionellen Komponenten zusammen. Der grau markierte Bereich kennzeichnet die Blöcke, die in der vorliegenden Arbeit erarbeitet und umgesetzt werden. Die wichtigsten Komponenten sind nachfolgend beschrieben.

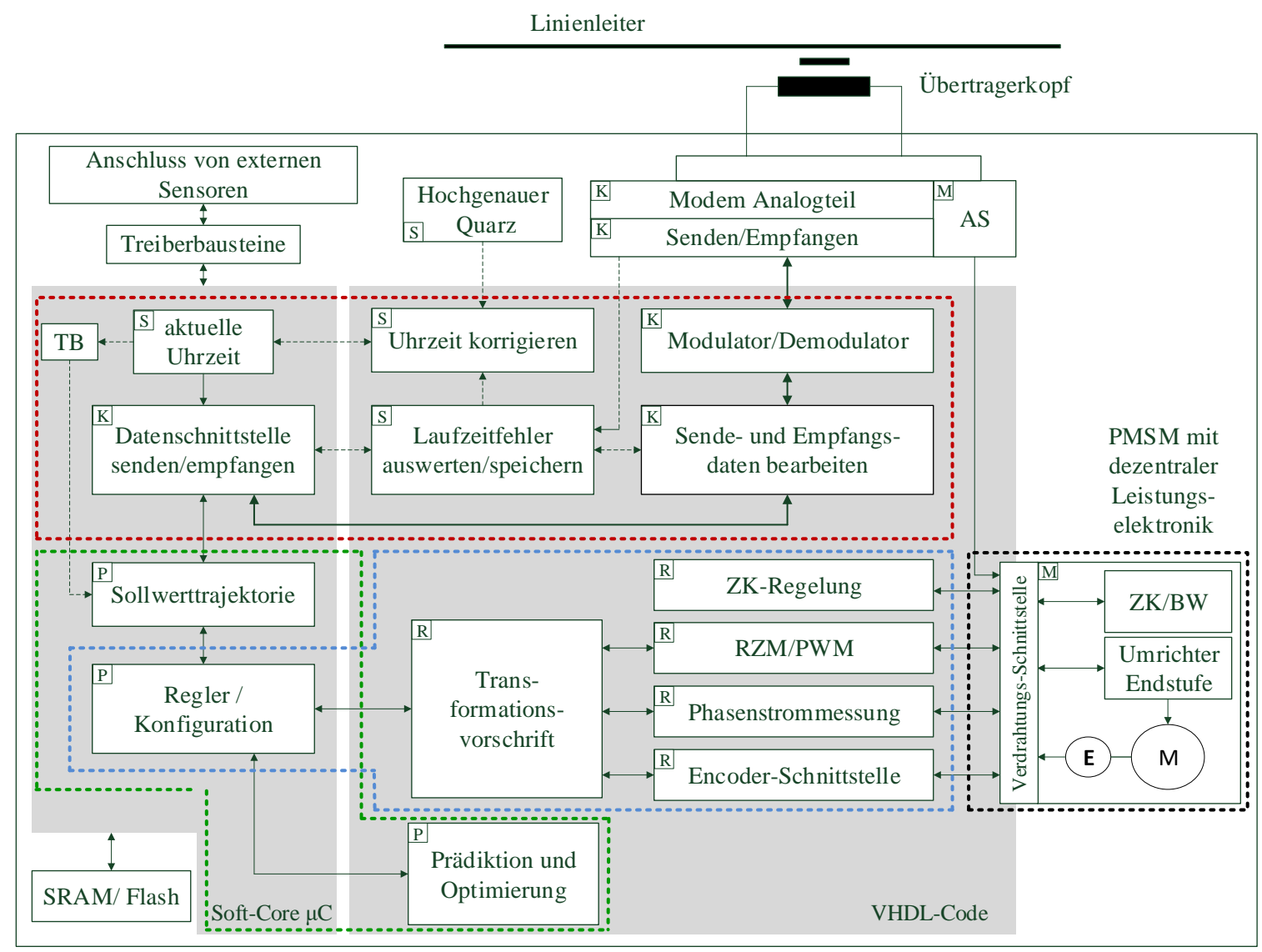

Abbildung 2.2.: Aufbau des intelligenten Aktors.

Die in der Abbildung 2.2 mit $K$ bezeichneten Funktionsblöcke kennzeichnen die Bereiche der Kommunikation. Die mit $S$ benannten Funktionsblöcke enthalten die Bereiche der Synchronisation, die auch für die Umsetzung des Zeitstempelverfahrens notwendig sind. TB (engl.: Time Base) bildet in der Darstellung die Basiszeit ab bzw. steht für die Synchronisierung und Generierung der Kommunikationsabläufe. Die mit $R$ bezeichneten Funktionsblöcke definieren die Bereiche der Regelung für die Ansteuerung der Leistungselektronik des Motors. P für Prädiktion kennzeichnet die Bereiche 
des prädiktiven Regelungsverfahrens, das entwickelt wird. Die $M$-Funktionsblöcke beinhalten die Bereiche der dezentralen Leistungselektronik zum Betrieb des Motors, speziell für den Betrieb einer permanentmagneterregten Synchronmaschine (PMSM, engl.: Permanent-Magnet Synchronous Motor).

Die einzige Verbindung des Aktors zur anbindenden Peripherie stellen der Übertragerkopf (vgl. Abbildung 3.7) und die optionalen lokal anschließbaren Sensoren dar. Im Energieübertragerkopf ist gleichzeitig der Übertragerkopf für das Sende- und Empfangsteil für die Datenübertragung integriert. Die Energieversorgung des Aktors und der Elektronikkomponenten selbst erfolgt autark über den Anpass-Steller (vgl. Abschnitt 3.1), in der Abbildung 2.2 mit $A S$ bezeichnet. Der beschriebene kompakte intelligente Aktor kann autark in Modulbauweise im Bereich der beweglichen elektrischen Verbraucher für verschiedenste Applikation zum Einsatz kommen. Auf die Funktionsblöcke bzw. Komponenten wird im nächsten Abschnitt näher eingegangen.

\section{2. Übersicht und Beschreibung der Komponenten}

Die in der Abbildung 2.2 dargestellten Funktionsblöcke haben unterschiedliche Aufgaben und werden im weiteren Verlauf näher erläutert. Durch das vorgestellte neue Konzept wird gezeigt, dass innerhalb eines SoPC-basierten Embedded Systems (SoPC, engl.: System on a Programmable Chip) ein sehr kompaktes Aktor- und Kommunikationsmodul im Bereich der mobilen Intralogistik und der beweglichen elektrischen Verbraucher realisiert wird.

Insgesamt lassen sich die dargestellten Funktionsblöcke in Abbildung 2.2 in vier übergeordnete Bereiche (rot, blau, schwarz und grün schraffierter Bereich) einteilen. Eine weitere Einteilung bzw. Zuordnung ergibt sich durch die Form der Code-Generierung im FPGA (engl.: Field Programmable Gate Array) (vgl. Abschnitt 5.1), entweder durch Synthetisierung in VHDL (engl.: Very High Speed Integrated Circuit Hardware Description Language) oder durch Programmierung im Soft-Core $\mu \mathrm{C}$ (vgl. Abschnitt 5.3.5).

Der erste übergeordnete Bereich umfasst den Bereich der Kommunikation $K$ und der Synchronisation $S$ (rot schraffierter Bereich in Abbildung 2.2). Der Ausgangspunkt für die Basis des Aktorkonzeptes bildet die Wahl des verwendeten Modems gemäß Abschnitt 4.3.1. Das Modem erlaubt die Kommunikation über den induktiven kontaktlosen Energieübertragungskanal, allerdings besteht keine Möglichkeit einer Synchronisation zwischen den Kommunikationsteilnehmern. Daher wird in einem ersten Schritt untersucht, ob die Modemfunktionalität so erweitert werden kann, dass eine Synchronisation zwischen den Teilnehmern durchführbar ist. 
Im Entwurf ist zu prüfen, ob es möglich ist, durch die Wahl eines geeigneten Übertragungsverfahrens eine Synchronisation zwischen den Teilnehmern über den stark störungsbehafteten induktiven Energieübertragungskanal mit dem vorgesehenen Zeitstempelverfahren zu realisieren. Dabei werden durch die Einbindung paralleler Abarbeitungsalgorithmen im Modem die Durchlaufzeiten der Signale innerhalb des Datenübertragungssystems bestimmt.

Die über den Übertragerkopf bzw. über den Analogteil des Modems gesendeten bzw. empfangenen Signale werden, wie in der Abbildung 2.2 vereinfacht dargestellt, vom Modulator bzw. Demulator auf einen geeigneten Frequenzbereich moduliert bzw. demoduliert. Der in der Abbildung 2.2 rot schraffierte Bereich beschreibt dabei neu zu erarbeitende und zusätzlich anzupassende Funktionsblöcke für die Realisierung der Kommunikation $K$ und der Datensynchronisation $S$. Die wichtigsten zu ergänzenden Funktionsblöcke betreffen die Auswertung des Laufzeitfehlers bei der Signalübertragung und die Verwaltung und Korrektur der lokalen Uhrzeit. Der Entwurf bzw. das entwickelte Verfahren wird ausführlich in Kapitel 4 beschrieben.

Die Basis der lokalen Uhrzeit für das Zeitstempelverfahren bildet ein hochgenauer Quarz-Baustein bzw. Oszillator. In Abschnitt 4.5 wird in einem Versuchsaufbau gezeigt, wie sich höherwertige Oszillatoren von Standardoszillatoren unterscheiden und welchen Einfluss unterschiedliche Oszillatorklassen auf die Synchronisationsvorgänge bei der Übertragung von Daten zwischen einzelnen Kommunikationsteilnehmern haben. Die dabei betrachtete Zwei-Oszillator-Methode ermöglicht im Versuchsaufbau die qualitative Bewertung der Ganggenauigkeit von Oszillatoren bei der Betrachtung gleicher Oszillatorklassen.

Um die Möglichkeit der Datenkommunikation und Teilnehmersynchronisierung unter realistischen Bedingungen zu testen und zu validieren, wurde in einem ersten Schritt ein Versuchsaufbau bzw. ein Funktionsmuster mit einer induktiven kontaktlosen Energieübertragung aufgebaut, welches die Ergebnisse der Datenkommunikation und Datensynchronisierung nachweist (vgl. Kapitel 3).

Der zweite übergeordnete Bereich in Abbildung 2.2 umfasst den Bereich der Regelungsplattform $R$ (blau schraffierter Bereich). Da die bestehende Struktur der Modem-Funktionalität auf einer SoPC-basierten Embedded System Plattform realisiert ist, die Technik einen leistungsfähigen, innovativen und auch bauraumspezifisch kompakten Ansatz bietet, wird für die Umsetzung des Steuerteiles des Aktors die gleiche Hardwareplattform gewählt. Mit SoPC-basierten Plattformen werden dabei variable und anpassbare elektronische Systemstrukturen aufgebaut. Durch die Verwendung FPGA-basierter Technik wird gezeigt, dass es möglich ist, ein sehr kompaktes und skalierbares Motorsteuerkonzept aufzubauen.

Durch die Verwendung der Hardwareplattform ist es möglich, einen motorischen Aktor in das Gesamtkonzept des intelligenten Aktors einzubinden und den Betrieb 
einer permanentmagneterregten Synchronmaschine in der Betriebsart feldorientierte Regelung zu ermöglichen.

In der Abbildung 2.2 sind die wesentlichen Funktionsblöcke abgebildet, die für den Betrieb einer PMSM in FOC benötigt werden. Neben dem Funktionsblock für die Transformationsvorschriften werden weitere Funktionsblöcke für die ZwischenkreisRegelung (ZK), Raumzeigermodulation (RZM) und Pulsweitenmodulation (PWM) sowie für die Encoder-Schnittstelle und die Phasenstrommessung benötigt. Alle Blöcke sind in VHDL-Code realisiert.

Bei der wichtigen Phasenstrommessung des Motors wird untersucht, ob es durch die Einbindung von modernen Strommess-Sensoren in Kombination mit FPGA-basierter Technik möglich ist, die Reaktionszeiten und die Genauigkeitsanforderungen für die Strommessung einfacher und effizienter zu realisieren. Die Entwicklung der FOC im FPGA ist ausführlich im Kapitel 5 beschrieben.

Der dritte in der Abbildung 2.2 mit $M$ für Motor bezeichnete schwarz schraffierte Bereich bildet den verwendeten Synchronmotor mit dazugehöriger dezentraler Leistungselektronik und Umrichter-Endstufe ab. ZK/BW bezeichnet den Zwischenkreis mit Bremswiderstand. Die Beschreibung der dezentraler Leistungselektronik ist im Abschnitt 5.2.1 zusammengestellt.

Der vierte und letzte grün schraffierte Bereich $P$ in Abbildung 2.2 steht für Prädiktion und umfasst den Bereich eines prädiktiven Regelungsverfahrens. Hier wird untersucht, ob es durch den Einsatz von prädiktiven Regelalgorithmen möglich ist, bessere Ergebnisse hinsichtlich der Regeleigenschaften für das Führungsverhalten und das Störverhalten der PMSM zu erreichen als bei klassischen Regelungsverfahren. Die regelungstechnische Betrachtung des intelligenten Aktors ist in Kapitel 6 beschrieben. Die mit $P$ bezeichneten Blöcke in Abbildung 2.2 für die Umsetzung des prädiktiven Regelungsverfahrens umfassen die Bereiche der Sollwerttrajektorie, der Regler-Konfiguration und der Prädiktions- und Optimierungsberechnungen.

Die Bezeichnung als smarter oder intelligenter Aktor bezieht sich in der vorliegenden Arbeit auf die Fähigkeit der Selbstversorgung des Aktors mit Energie über den Anpass-Steller, einer unterlagerten integrierten Kommunikation ohne zusätzliche Schnittstelle und die Einbindung komplexer Abläufe für die Kommunikation. Ein weiteres Herausstellungsmerkmal ist die Integration von hardwarebasierten Regelungsalgorithmen und die Einbindung eines prädiktiven Regelungskonzeptes.

Um die Eigenschaften des Energiekanals hinsichtlich der Datenkommunikation zu analysieren und zu bewerten, wird im ersten Schritt, wie bereits dargelegt, ein Versuchsaufbau mit einem induktiven kontaktlosen Energieübertragungssystem realisiert. Im zweiten Schritt dient der Versuchsaufbau bzw. das Funktionsmuster dazu, das erarbeitete Kommunikationskonzept zu validieren. Als Funktionsmuster wurde die Nachbildung einer Rundläufer-Etikettiermaschine gewählt. 
Zusammenfassend, entsprechend den genannten Aufgaben laut Abschnitt 1.3, wird in der vorliegenden Arbeit folgende Vorgehensweise zum Erreichen der Ziele verfolgt:

- Kapitel 3: Aufbau eines Versuchs- bzw. Funktionsmusters mit induktiver kontaktloser Energieübertragung zur Validierung der Datenkommunikation und datentechnischen Synchronisation zwischen den Teilnehmern

- Kapitel 4: Realisierung eines Verfahrens für die Datenkommunikation und Synchronisation zwischen den Teilnehmern (entspricht dem rot schraffierten Bereich in Abbildung 2.2)

- Kapitel 5: Erstellung und Aufbau einer neuen Plattform für kompakte intelligente Aktoren (entspricht dem blau schraffierten Bereich in Abbildung 2.2)

- Kapitel 6: Entwicklung und Untersuchung eines Regelungskonzeptes mit prädiktivem Verhalten (entspricht dem grün schraffierten Bereich in Abbildung $2.2)$.

Das nachfolgende Kapitel 3 beschreibt den Aufbau des Funktionsmusters. Entsprechend dem Funktionsprinzip einer Etikettiermaschine sind alle relevanten Bewegungsabläufe der Etikettiermaschine nachgebildet. Eine Etikettiermaschine stellt erhöhte Anforderungen an den mechanischen Aufbau und die steuerungstechnische Realisierung. Dabei ist eine mechanische und elektrische Kompaktheit gefordert. Durch die notwendige Synchronisation zwischen den Antrieben und den gegebenen dynamischen Lastwechseln werden an die Antriebe gleichfalls hohe Anforderungen gestellt.

Ein weiterer wichtiger Punkt betrifft den Datenaustausch zwischen den Teilnehmern. Durch die notwendige Synchronität und der hohen Dynamik zwischen den unterschiedlichen Antrieben entsteht ein hohes Datenaufkommen auf dem Kommunikationskanal, das Echtzeitbedingungen genügen muss. 



\section{Aufbau eines Funktionsmusters mit kontaktloser Energieübertragung}

Die in Kapitel 4 durchgeführten Untersuchungen betreffend der Datenübertragung und der Synchronisierung zwischen den stationären und beweglichen elektrischen Verbrauchern im Bereich der induktiven kontaktlosen Energieübertragung wurden auf dem nachfolgenden beschriebenen Funktionsmuster einer Rundläufer-Etikettiermaschine durchgeführt. In dem entwickelten Funktionsmuster ist der Aufbau einer induktiven kontaktlosen Energie- und Datenübertragung integriert und realisiert. Damit wird gezeigt, wie ein solches neuartiges konzeptioniertes System, betreffend der Linienleiterführung und Datenanbindung innerhalb der Applikation, für eine drehbare bzw. rotative Prozessbewegung aufgebaut ist.

Der Aufbau des Funktionsmusters besteht im Kern aus zwei Teilen. Zum einen aus einer feststehenden (stationären) Komponente, die den Sockel bzw. die Tragkonstruktion bildet und zum anderen aus einem drehbaren (beweglichen) Teil, der auf der Tragkonstruktion aufbaut bzw. aufsitzt. Der Energieversorgungskanal dient gleichzeitig als Übertragungskanal für die Datenanbindung zu den einzelnen beweglichen Teilnehmern. Die kontaktlose Datenübertragung über den induktiven Energieübertragungskanal wird dabei unter realen Bedingungen am Versuchsaufbau überprüft, d.h. unter Einbindung möglicher Störszenarien wie Einschalt- und Ausschaltvorgänge der Maschine, Lastwechselspiele, Umweltbedingungen wie Temperatur, EMV (Elektromagnetische Verträglichkeit) und anderer Faktoren.

Im folgenden Abschnitt wird konkret auf den Aufbau des realisierten induktiven kontaktlosen Energieübertragungssystems eingegangen. Im weiteren Verlauf wird eine Etikettiermaschine beschrieben, wie sie in der Industrie eingesetzt wird. Die Komponenten und der Funktionsablauf einer Rundläufer-Etikettiermaschine werden erläutert. Die nächsten Abschnitte beschreiben im einzelnen den mechanischen und elektrischen Aufbau des Funktionsmusters mit seinen Baugruppen. Im letzten Abschnitt werden die Eigenschaften und die Störeinflüsse des Datenübertragungskanals über den induktiven kontaktlosen Energieübertragungskanal analysiert und dargestellt. 
Die Analyse des Datenkanals ist eine wichtige Voraussetzung für die in Kapitel 4 beschriebenen Untersuchungen.

\subsection{Komponenten der kontaktlosen Energieübertragung}

In der vorliegenden Arbeit wurde für den experimentellen Aufbau der Energieversorgung des Funktionsmusters einer Etikettiermaschine das System MOVITRANSB der Fa. SEW-Eurodrive verwendet, das zur induktiven kontaktlosen Energieübertragung im industriellen Bereich eingesetzt wird. Auf den technischen Aufbau einer solchen Energieversorgung soll im Abschnitt näher eingegangen werden. Weiterführende Informationen finden sich in [182], [200], [197]. Den prinzipiellen Aufbau des induktiven kontaktlosen Energieübertragungssystems zeigt Abbildung 3.1.

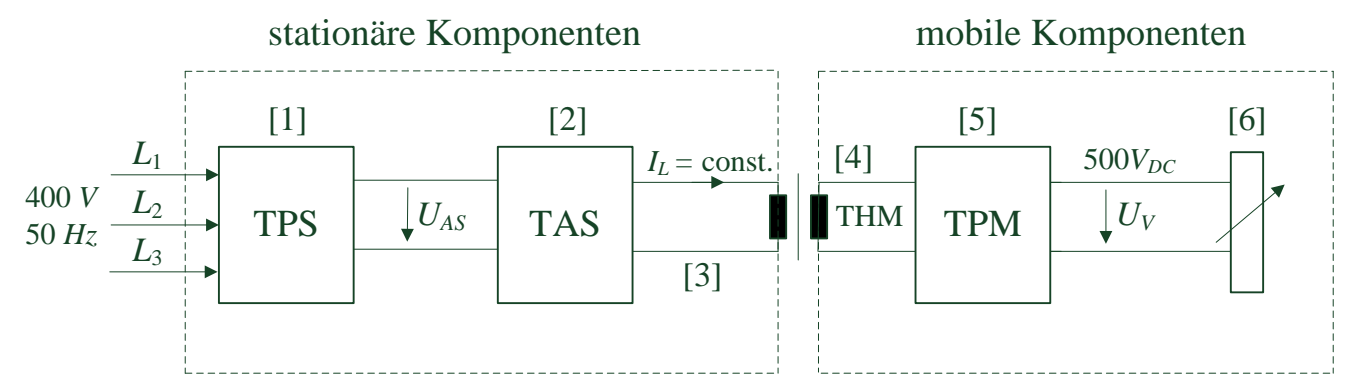

Abbildung 3.1.: Aufbau eines kontaktlosen Energieübertragungsystems [182].

[1] TPS (Einspeise-Steller), [2] TAS (Anschaltmodul), [3] Übertragerstrecke (Linienleiter), [4] THM (Übertragerkopf), [5] TPM (AnpassSteller), [6] Mobile Verbraucher.

Beim TPS (Einspeise-Steller) handelt es sich funktionell um einen Frequenzumrichter. Er wandelt den netzseitigen dreiphasigen Drehstrom mit einer Frequenz von $50 \mathrm{~Hz}$ zunächst in eine Gleichspannung (AC/DC-Wandlung) um und danach in eine einphasige rechteckige Wechselspannung $U_{A S}$ mit einer konstanten Frequenz von $25 \mathrm{kHz}$ (DC/AC-Wandlung). Der Einspeise-Steller stellt dem nachgeschaltetem TAS (Anschaltmodul), gleichfalls eine stationäre Komponente, die Ausgangsspannung $U_{A S}$ zur Verfügung. Abbildung 3.2 zeigt den internen prinzipiellen Aufbau des Anschaltmoduls.

Er besteht im wesentlichen aus drei funktionalen Teilen, dem Gyrator, dem AnpassTransformator und dem Kompensationsmodul. Den Kern des Gyrators bildet ein Schwingkreis aus einer Spule $L_{G}$ und einem Kondensator $C_{G}$, deren Resonanzfrequenz 


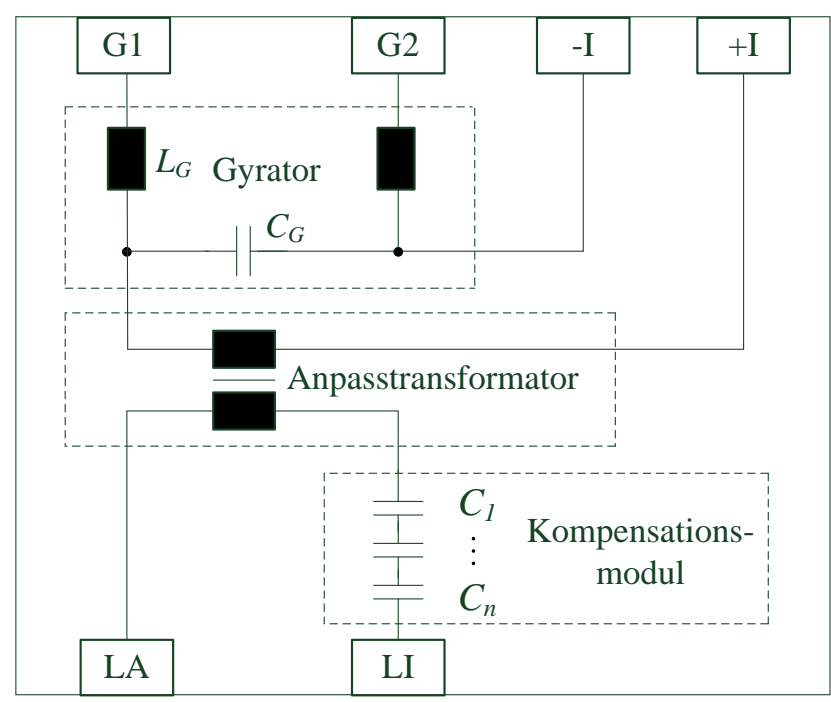

Abbildung 3.2.: Aufbau des Anschaltmoduls [182].

auf einen Wert von $25 \mathrm{kHz}$ ausgelegt wird. Die Gyratorimpedanz $Z_{G}$ berechnet sich gemäß Gl. (3.1) [182].

$$
Z_{G}=\sqrt{\frac{L_{G}}{C_{G}}} .
$$

$L_{G}$ steht für die Induktivität des Gyrators in $\mu H, C_{G}$ ist die Kapazität des Gyrators in $\mu F$. Für den Gyrator-Ausgangsstrom $I_{G}$ gilt der Zusammenhang laut Gl. (3.2). $U_{A S}$ ist die Ausgangsspannung in $V$.

$$
I_{G}=\frac{U_{A S}}{Z_{G}}
$$

Die zentrale Aufgabe des Gyrators ${ }^{1}$ ist dabei die eingangsseitige Spannungsquelle in eine ausgangsseitig spannungsgesteuerte Konstantstromquelle umzuwandeln. Durch das Einprägen eines konstanten Linienleiterstromes $I_{L}$ ist gewährleistet, dass entlang des in der Anlage verlegten Linienleiters überall das gleiche Magnetfeld entsteht und die gleiche Leistung bereitgestellt werden kann. Der nachgeschaltete AnpassTransformator trennt den Linienleiter galvanisch zu der versorgenden Leistungselektronik und passt den Linienleiterstrom durch ein entsprechendes Übersetzungsverhältnis den konstruktiven Anforderungen an. Das kapazitive Kompensationsmodul kompensiert die Induktivität des Linienleiters, wodurch die Blindleistungsaufnahme der Energieübertragungsstrecke reduziert wird.

${ }^{1}$ auch als Dualinverter bezeichnet 
Der Induktionsbelag des Linienleiters hat, vor allem bei langen Versorgungsstrecken, neben dem beschriebenen Blindleistungsbedarf ferner zur Folge, dass auch das elektrische Potential über die Linienleiterlänge ansteigt. In einem solchen Fall muss zusätzlich noch eine Kompensation entlang der Strecke durchgeführt werden.

Über den Linienleiter und einem Luftspalt von $10 \ldots 20 \mathrm{~mm}$ wird schließlich die Energie kontaktlos auf den mobilen Übertragerkopf übertragen. Das TPM (AnpassSteller) wandelt, als letztes Glied in der Energieübertragungskette, die eingeprägte Spannung des Übertragerkopfes in eine Gleichspannung $U_{V}$ von $500 \mathrm{~V}$ um. Die Spannung kann dann direkt den Zwischenkreis eines Frequenzumrichters versorgen oder anderen mobilen Verbrauchern als Eingangsspannung zur Verfügung gestellt werden.

\subsection{Etikettieranlagen in der Industrie}

Der Versuchsaufbau gemäß Abschnitt 3.3 bildet ein Funktionsmuster einer Rundläufer-Etikettiermaschine nach. Kontinuierlich arbeitende Rundläufer-Etikettiermaschinen werden für eine Ausbringung von 50 ..2.000 Flaschen pro Minute angeboten $[72]$.

Die Anforderungen an den Etikettierprozess sind komplex. Das Etikett muss punktgenau an der definierten Stelle der Flasche oder des zu etikettierenden Behältnisses bei unterschiedlichen hohen Produktionsgeschwindigkeiten aufgebracht werden. Falten oder Beschädigungen des Etiketts müssen ausgeschlossen werden. Weitere Anforderungen an die Etikettiermaschine sind u.a. ein robuster Aufbau, kurze Umstellzeiten bei unterschiedlichen Behälter- und Etikettenformaten sowie ein minimaler Wartungsaufwand der Aggregate und Maschinenkomponenten.

Etikettiermaschinen lassen sich prinzipiell durch ihre Arbeitsweise unterscheiden. Zirkular arbeitende Etikettiermaschinen, auch als Rundläufer-Etikettiermaschine bezeichnet, werden im oberen Leistungsbereich eingesetzt. Dabei sind bis zu 2.000 Etikettiervorgänge pro Minute möglich [72]. Durch die Fixierung des zu etikettierenden Behälters auf dem Behälterteller ist ein besonders faltenfreier und exakter Etikettensitz möglich. Linear arbeitende Etikettiermaschinen, auch als GeradläuferEtikettiermaschine bezeichnet, sind in der Regel günstiger und kommen im unteren Leistungsbereich zum Einsatz [72].

Je nach Art der Etiketten, wie z.B. Rundum-, Brust- oder Rückenetikett, kommen dabei unterschiedliche Etikettierverfahren zum Einsatz. Auch bei der Beleimung der Etiketten gibt es unterschiedliche Abläufe wie z.B. das Kaltleim-, Heißleim- und 


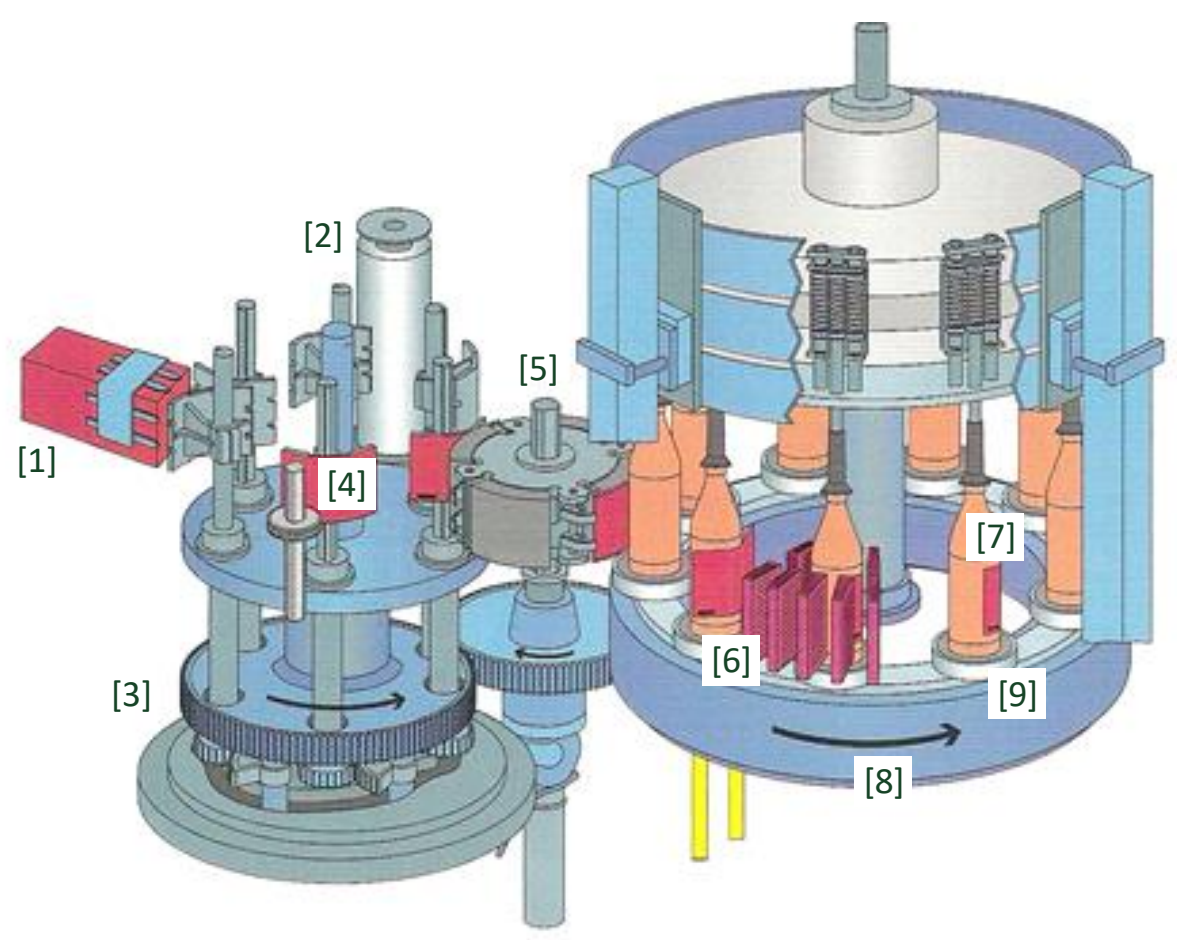

Abbildung 3.3.: Darstellung einer industriellen Etikettiermaschine [114].

[1] Etikettierkasten, [2] Leimwalze, [3] Stationsgehäuse, [4] Leimsegment, [5] Greiferzylinder, [6] Bürsten, [7] Flasche/Behälter, [8] Flaschenträger/Behälterdrehtisch, [9] Drehteller.

Selbstklebeetikettierungs-Verfahren. Weitere ausführliche Informationen zu Etikettiermaschinen und Anlagen finden sich in [114]. Abbildung 3.3 zeigt den prinzipiellen Aufbau einer Rundläufer-Etikettiermaschine.

Die Flaschen werden dem rotierenden Behälterdrehtisch zugeführt und auf jedem Drehteller durch eine Zentrierglocke bzw. eine Haltevorrichtung fixiert. Größere Rundläufer-Etikettiermaschinen können mit mehreren Dutzend solcher Drehteller ausgestattet sein. Während der Drehbewegung des Behälterdrehtisches wird das mit Leim versehene Etikett auf den Behälter angedrückt und nach leichter Drehung des Drehtellers durch außen fest angebrachte Bürsten vollständig auf die Behälteroberfläche aufgebürstet. Die Ausrichtung eines jeden Drehtellers während des Etikettiervorganges kann dabei mechanisch synchronisiert sein, z.B. durch Führung des Behältertisches über Drehkreuze und Kurvensegmente (Formbehälter) oder durch Kopplung der Behälterteller mittels Zahnriemen (Rundbehälter) [114].

Für spezielle Ausstattungen, wie z.B. bei geforderter vorheriger Behälterausrichtung, ist auch eine elektronische Synchronisation durch Ansteuerung der einzelnen Dreh- 
tellermotoren möglich. Jeder Drehtellermotor wird dabei über einen separaten umrichtergesteuerten Servomotor angesteuert. Die Kommunikation der Umrichter untereinander wird dabei in der Regel dezentral über eine Feldbusschnittstelle realisiert. Eine solche Struktur wurde beim Aufbau des Funktionsmusters aufgenommen und umgesetzt.

\subsection{Funktionsmuster einer Etikettiermaschine}

Abbildung 3.4 zeigt die konstruktive Umsetzung bzw. den fertigen Aufbau des Funktionsmusters einer Etikettiermaschine für die in Kapitel 4 beschriebenen Untersuchungen. Auf der oberen Holzplatte sind die aufgebauten dezentralen Antriebssteuerungen mit den zugehörigen Drehtellermotoren zu erkennen. Nachfolgend werden der Aufbau und die wesentlichen verwendeten Komponenten beschrieben. Speziell der Aufbau des induktiven kontaktlosen Energieübertragungssystems zu den beweglichen Komponenten bildet die Basis für die Versuchsdurchführung im nächsten Kapitel.

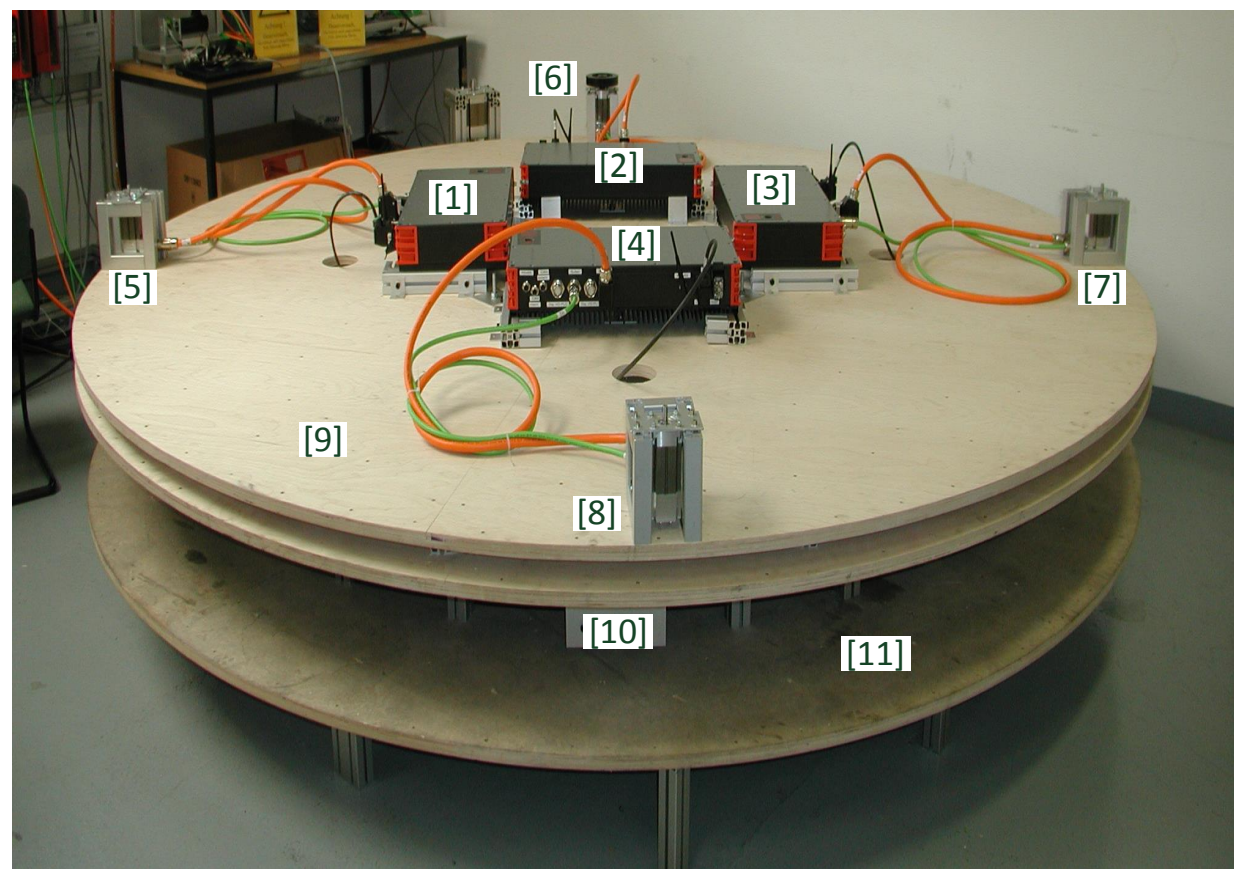

Abbildung 3.4.: Aufgebautes Funktionsmuster einer Etikettiermaschine. [1-4] dez. Steuerungen für die Drehtellermotoren, [5-8] Drehtellermotoren 1-4, [9] beweglicher Teil, [10] Bereich der kontaktlosen Energieu. Datenübertragung, [11] stationärer Teil. 
Der komplette Ablauf des Etikettiervorgangs für den konkreten Aufbau des Funktionsmusters der Etikettiermaschine ist in Abbildung 3.5 dargestellt. An der Station A

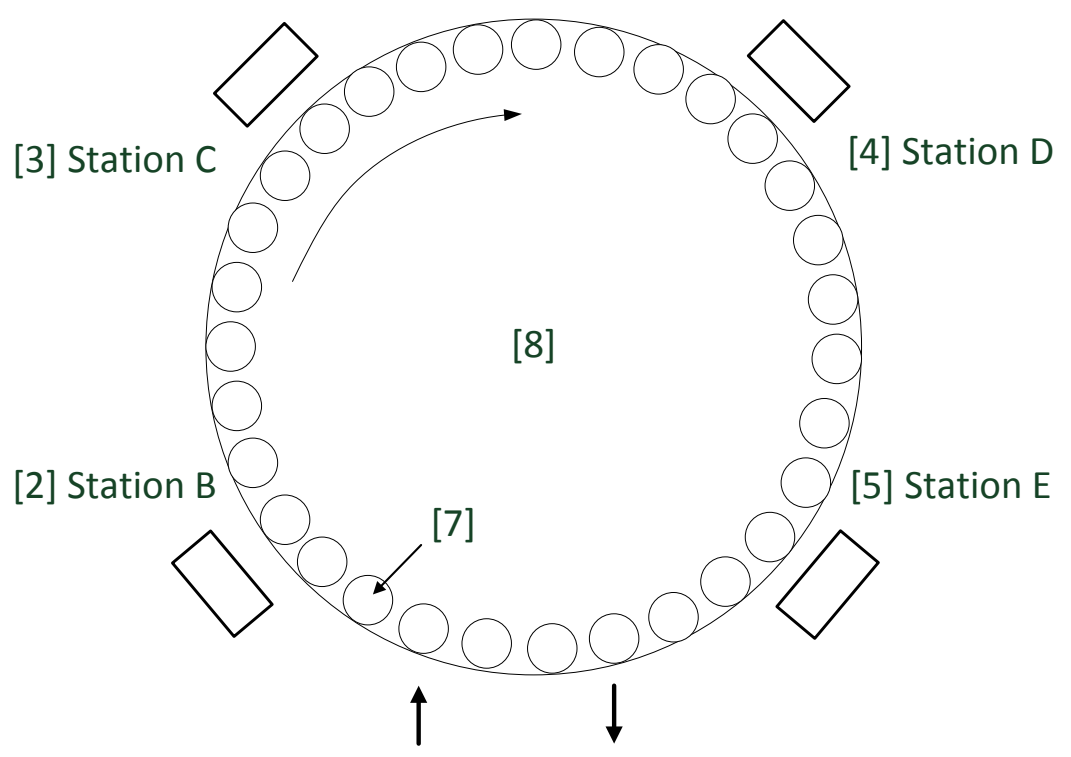

[1] Station A [6] Station F

Abbildung 3.5.: Bewegungsablauf beim Etikettiervorgang.

[1] Bereich der Behälterzuführung, [2] Station für die Markierungserkennung, [3] Etikettierstation 1, [4] Etikettierstation 2, [5] Qualitätskontrolle, [6] Bereich der Behälterrückführung, [7] Behälterdrehteller, [8] Behälterdrehtisch.

werden die Behälter zuerst der Etikettiermaschine bzw. den einzelnen Drehtellern auf dem Drehtisch zugeführt. Der Drehtisch transportiert die Behälter, bei gleichzeitiger Drehung der einzelnen Drehteller, an der Station B für die Markierungserkennung vorbei. Nach der Abtastung der Markierung auf dem Behälter wird der Steuerung des gerade abgetasteten Drehtellers die aktuelle Position der Markierung, bezogen auf den eingespannten Zustand des Behälters, mitgeteilt.

Die Drehtellersteuerung kann den Behälter nun entsprechend der bekannten Markierungsposition ausrichten. An der Etikettierstation C (z. B. Vorderetikett) bzw. an der Etikettierstation D (z. B Rück- oder Halsetikett) findet schließlich die Etikettierung des Behälters statt. Wichtig ist, dass die Bewegungen der einzelnen Aggregate, d.h. die Bewegungen der einzelnen Drehteller zu den Etikettierstationen synchronisiert ablaufen, um Falten- und Rissbildung während des Etikettiervorgangs zu vermeiden. Die Synchronisierung ist für unterschiedliche Produktionsgeschwindigkeiten, für das Hochfahren der Anlage und bei Abschalten der Anlage zu gewährleisten. 
Bevor die Behälter wieder an der Station F entnommen werden können, findet an der Station E die Qualitätskontrolle statt. Die Behälteretiketten werden mithilfe von optischen Systemen auf Korrektheit überprüft, wie z.B. auf Position, Drehlage, Richtigkeit des Etikettes. Auch andere Merkmale des Behälters wie z.B. Füllhöhe und Behälterverschluss können überprüft werden. Erkannte fehlerhafte Behälter werden aussortiert. Auf die Beschreibung und die Umsetzung der einzelnen Aggregate sowie auf die detaillierten Bewegungsabläufe während des Etikettiervorgangs soll an der Stelle nicht weiter eingegangen werden.

\subsection{Aufbau des Funktionsmusters}

Das im Rahmen dieser Arbeit entwicklelte Funktionsmusters setzt sich zusammen aus einem stationären (feststehenden) Teil und aus einem beweglichen (drehbaren) Teil. Die einzelnen Bereiche werden nachfolgend beschrieben.

\subsubsection{Stationärer mechanischer Aufbau des Funktionsmusters}

Der stationäre Aufbau des Funktionsmusters ist in Abbildung 3.6 dargestellt. Die Tragkonstruktion besteht aus einem Gestell aus Aluminiumprofilen und dem Sockel eines Maschinendrehkranzes. Eine Leimholzplatte verbindet beide Teile fest miteinander, was für eine ausreichende Steifigkeit des Aufbaus sorgt. Der Antriebsmotor des Drehtisches ist über eine spezielle Aufnahme- und Arretierungsvorrichtung im Drehkranz integriert. Beide Komponenten sind mittig im Untergestell eingebaut und bilden den Drehpunkt des Drehtisches. Die Leimholzplatte bildet die Unterkonstruktion für den stationären Teil des kontaktlosen Energieübertragungssystems (siehe auch Abschnitt 3.1). Die Umsetzung und Realisierung stellt einen wichtigen und zentralen Teil der Arbeit innerhalb des Aufbaus des Funktionsmusters dar.

Abbildung 3.7 zeigt den Aufbau und die Verlegung des Linienleiters. Der Linienleiter ist als feindrahtige Mittelfrequenzlitze ausgeführt und auf die Frequenz des Linienleiterstromes von $25 \mathrm{kHz}$ ausgelegt. Eingangsseitig kann der Linienleiterstrom auf einen maximalen Strom von $60 A$ oder $85 A$ eingestellt werden. Für den konkreten Versuchsaufbau bzw. Testbetrieb wurden $60 A$ Eingangsstrom eingestellt.

Zur Aufnahme und für die Verlegung der Linienleiter sind speziell geschlitzte Profilleisten entlang des Umfangs des Drehtisches eingebaut. Im mittleren Teil der Profilleiste sind zwei Linienleiter, von der Einspeiseseite her kommend (Hinleiter), geführt. Die Linienleiter sind am Ende der Übertragerstrecke aufgeteilt und getrennt voneinander wieder im oberen und unteren Bereich der Profilleiste (Abbildung 3.7) zurückgeführt (Rückleiter). Durch die neuartige Linienleiterverlegung am Versuchsaufbau 


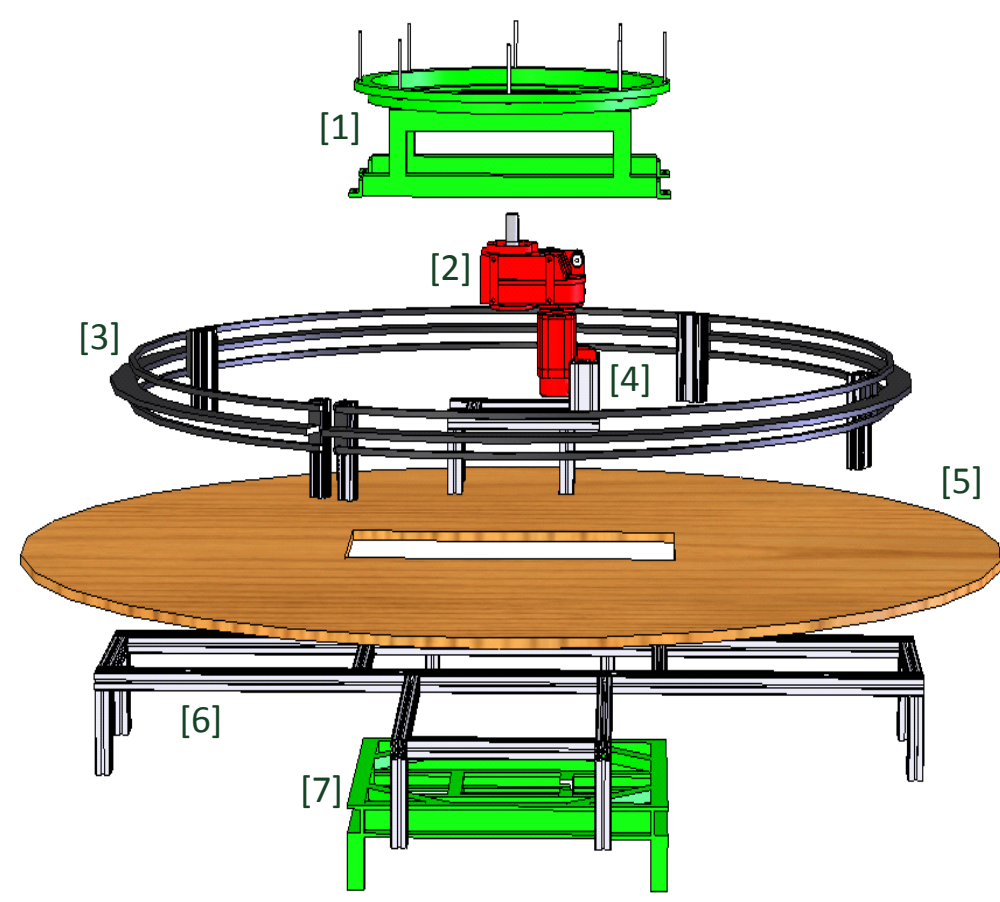

Abbildung 3.6.: Aufbau des stationären Teils des Funktionsmusters.

[1] Drehkranz, [2] Drehtischmotor, [3] Übertragerstrecke, [4] Haltevorrichtung, [5] Leimholzplatte, [6] Gestell, [7] Sockel.

wird erreicht, dass die Übertragungsstrecke eine definierte Induktivität je aufgebauten Streckenmeter aufweist.

Beim Aufbau der Übertragungsstrecke war ferner zu berücksichtigen, dass die übertragbare Leistung über den kompletten Streckenverlauf konstant bleibt (Kompensation) und die Strecke keine (mechanische) Lücke aufweist. Ferner war darauf zu achten, dass keine Eisen- bzw. ferromagnetische Materialien für die Montage oder sonstiger Anwendung im Bereich der Übertragungsstrecke verwendet sind. Ferromagnetische Materialien können die Induktivität der Übertragerstrecke stark beeinflussen und sich vor allem im Bereich des Übertragerkopfes (Resonanzbereich) stark erwärmen. Als Montagematerial wurden deshalb überwiegend nicht ferromagnetische Materialien wie Edelstahl oder Aluminium verwendet. 


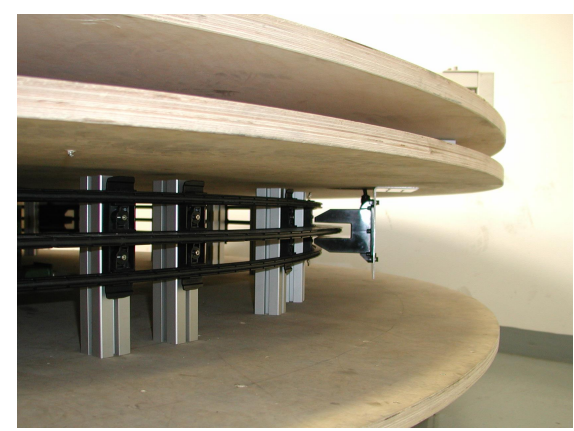

(a) Montage und Führung.

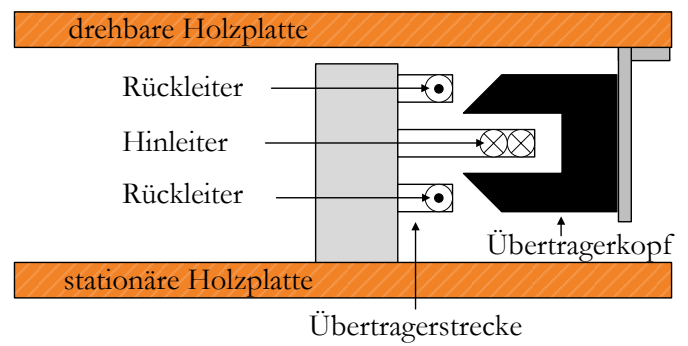

(b) Schnittzeichnung.

Abbildung 3.7.: Montage des U-Übertragerkopfes am Funktionsmuster.

\subsubsection{Beweglicher mechanischer Aufbau des Funktionsmusters}

In Abbildung 3.4 ist der bewegliche Teil des Funktionsmusters zu erkennen. Die unterste drehbare Leimholzplatte (2. Platte von oben) ist fest mit dem Maschinendrehkranz verschraubt. Die oberste Platte dient zur Aufnahme der Antriebsumrichter. Beide Platten sind durch ein Gestell aus Aluminiumprofilen fest miteinander verbunden.

Der Durchmesser der obersten Platte für die Aufnahme der Drehtellermotoren beträgt ca. $3 \mathrm{~m}$ - was eine mögliche Etikettierkapazität bei Nennbetrieb von ca. 70 Tsd. Behältern/Flaschen pro Stunde ermöglicht. Die Kapazitätsauslegung gilt bei Verwendung entsprechender Drehteller mit einem Durchmesser von ca. $80 \mathrm{~mm}$, einer Etikettierstrecke von $50 \mathrm{~mm}$ und einer Etikettenlänge von $100 \mathrm{~mm}$.

An der Unterseite der unteren Platte ist für jeden Drehtellerantrieb ein Übertragerkopf (Abbildung 3.7) montiert, sie versorgen die einzelnen beweglichen Teilnehmer jeweils separat mit Energie. Die maximale übertragbare Leistung eines Übertragerkopfes liegt bei ca. $800 \mathrm{~W}$, was für die Anwendung aber nicht benötigt wird.

\subsubsection{Stationärer elektrischer Aufbau des Funktionsmusters}

Für die Bereiche Energieversorgung und Steuerungstechnik bzw. Kommunikation zwischen den Teilnehmern lässt sich, wie beim mechanischen Aufbau, eine Unterscheidung in den stationären und beweglichen Teil treffen. Durch den Aufbau der kontaktlosen Energieversorgung zum beweglichen Teil sind beide Seiten, betreffend Energieversorgung und Kommunikationsanbindung, galvanisch voneinander getrennt.

Abbildung 3.8 zeigt den prinzipiellen elektrischen Aufbau mit den wichtigsten Komponenten für den stationären Teil der Energieversorgung und der Kommunikation 
des Funktionsmusters. Die Spannungsversorgungsebene ist im Blockschaltbild nicht berücksichtigt. Der Aufbau des induktiven kontaktlosen Energieübertragungssystems wurde, wie bereits erwähnt, mit dem System MOVITRANS realisiert [197].

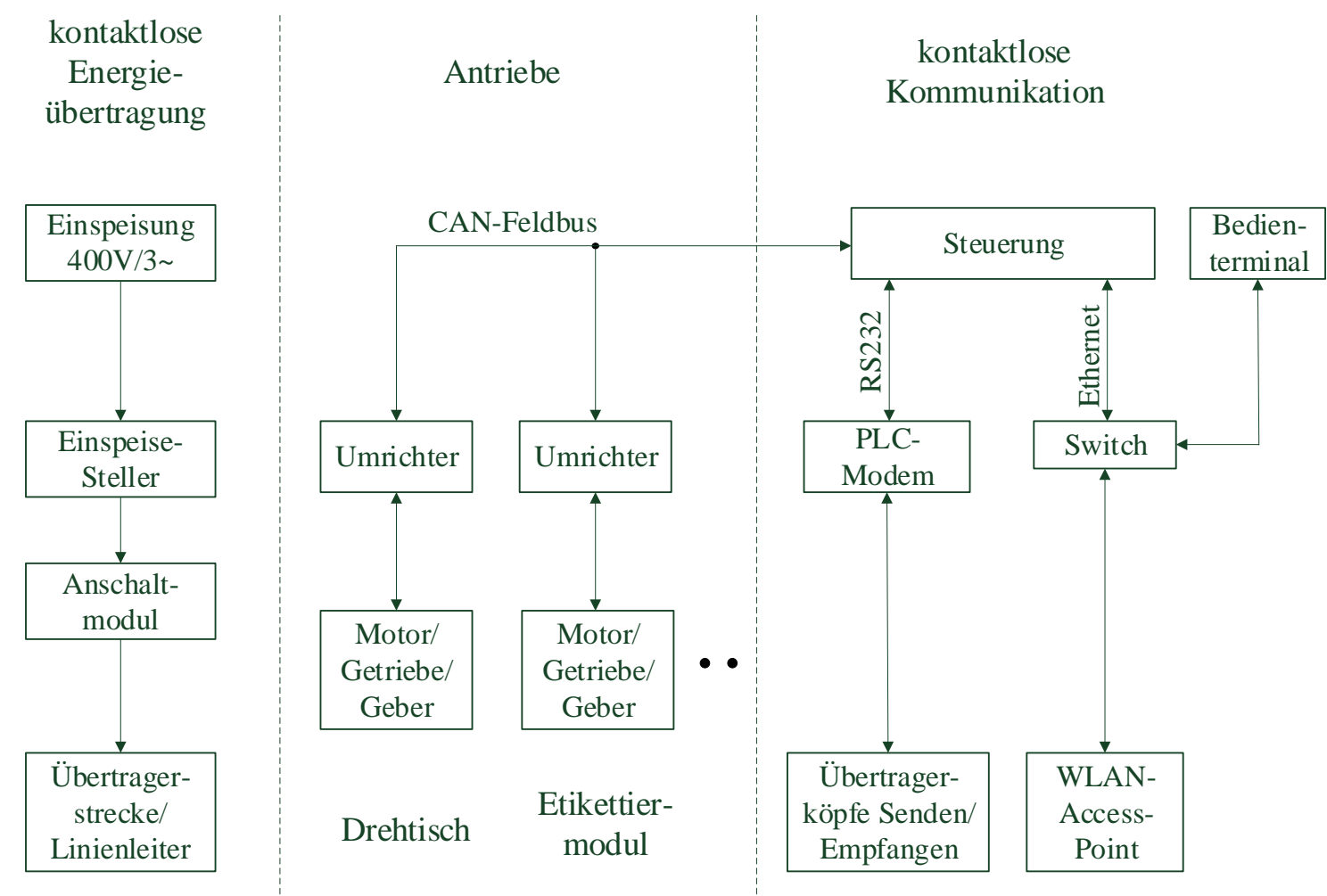

Schnittstelle zum beweglichen Teil

Abbildung 3.8.: Blockschaltbild der stationären elektrischen Komponenten.

Die Energieübertragungsstrecke bzw. der Linienleiter wird vom Einspeise-Steller bzw. vom Anschaltmodul versorgt [198]. Der eingeprägte konstante Wechselstrom (Konstantstromquelle) ist auf einen Wert von $60 \mathrm{~A}$ eingestellt. Auf der stationären Seite sind im ersten Schritt zwei Umrichter zur Ansteuerung des Drehtischmotors und des ersten Etikettiermoduls aufgebaut. Beide Umrichter sind über einen CAN-basierten Feldbus [56], [185] mit der Steuerung verbunden. Die Programmierung der Steuerung ist mit der Software MOVITOOLS-MotionStudioß [195] realisiert, hier besteht die Möglichkeit, auf eine vorhandene Bibliothek mit entsprechenden Funktionsblöcken und Funktionsbausteinen zurückzugreifen. Betreffend dem Datenaustausch zwischen beiden Seiten wurde im ersten Schritt eine WLAN-Kommunikation aufgebaut, die für allgemeine Ansteuer- und Diagnosefunktionen verwendet wird. Als Sende- und Empfangsstation für das Funknetzwerk zum beweglichen Teil wird ein Access Point ${ }^{2}$

\footnotetext{
${ }^{2}$ Basisstation für ein drahtloses lokales Netzwerk
} 
(AP) verwendet. Der AP ist über einen Switch über ein ethernetbasiertes Netzwerk mit der Steuerung verbunden. Auf das Funknetzwerk soll an der Stelle nicht detaillierter eingegangen werden [202].

Parallel zur Funkübertragung wurde die kontaktlose Kommunikation über den Linienleiter aufgebaut (siehe Kapitel 4). Neben dem WLAN-Access-Point ist weiterhin ein Bedienterminal (DOP11B-50) im Netzwerk integriert [201]. Mit dem Bedienterminal können wichtige Betriebszustände ausgelesen werden oder es besteht die Möglichkeit, einzelne Komponenten zu- oder abzuschalten. Weiter ist es möglich, am Switch einen PC zu Inbetriebnahme- und Diagnosezwecke anzuschließen.

Abbildung 3.9 beschreibt den Aufbau und die verwendeten Baugruppen der stationären Seite. Die Schaltschrankkomponenten sind auf einem Lochblech befestigt, dessen Rahmen aus einem Aluminiumgestell besteht. Die stationäre SPS ist im Optionsschacht des Umrichters für den Drehtischmotor integriert (Pos. [5]). Die Spannungsversorgung und die Kommunikation zum Umrichter sind über Steckkontakte auf dem SPS-Board gewährleistet. Not-Aus-Kreis und Sicherheitsschaltung sind zweikanalig ausgeführt.

\subsubsection{Beweglicher elektrischer Aufbau des Funktionsmusters}

Das Blockschaltbild für den Aufbau der einzelnen beweglichen Komponenten ist in Abbildung 3.10 dargestellt. Das umgesetzte Konzept in der Arbeit sieht vor, dass jeder Drehtellermotor seine eigene Energieversorgung und seine eigene Kommunikationsanbindung erhält. Der Anteil der notwendigen Bauteile erhöht sich zwar gegenüber einer zentralen Energie- und Kommunikationsanbindung, die Vorteile dafür sind aber ein geringer Installationsaufwand und vor allem eine höhere Flexibilität hinsichtlich Erweiterbarkeit und Austauschbarkeit der Module.

Jedes Modul bzw. jeder Drehtellermotor wird durch einen eigenen Umrichter angetrieben. Die Energieversorgung für den Umrichter stellt der Anpass-Steller [197] zur Verfügung. Er nimmt die Energie über den Übertragerkopf [199] auf und speist direkt in den Spannungszwischenkreis des Umrichters $\left(500 V_{D C}\right)$. Beim aktuellen Aufbau kommuniziert jeder Umrichter mit seiner eigenen Steuerung. Die Steuerung dient dabei auch als Schnittstellenumsetzer zum WLAN-Client bzw. zum PLC-Modem bei Betrieb über den Linienleiter (siehe Abbildung 4.7). Ziel ist es, in einem weiteren Schritt die aufgeführten funktionellen Anforderungen des Moduls in eine kompakte Baugruppe zu überführen.

Der Aufbau der dezentralen Antriebssteuerung bzw. des Antriebsmoduls für die einzelnen Drehtellermotoren wurde mit dem modularen Baukastensystem MOVIPROB [203] realisiert. Abbildung 3.11 zeigt den Aufbau des verwendeten Moduls. Es zeichnet sich durch ein modulares Gehäusekonzept aus und kann mit unterschiedlichen 


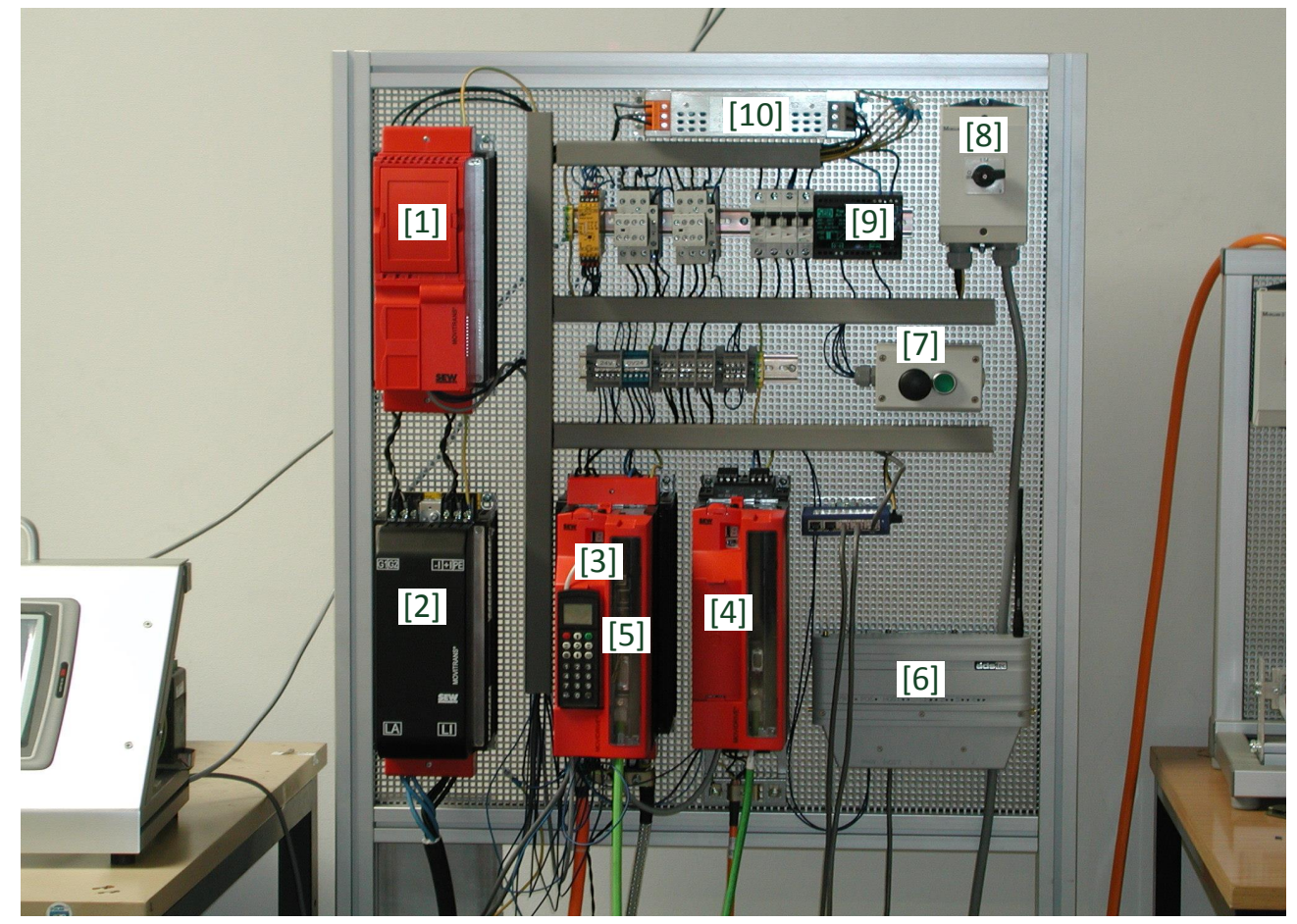

Abbildung 3.9.: Aufbaubild Schaltschrank für die stationäre Seite.

[1] Einspeise-Steller TPS10A040-NF0-503-1 [198], [2] Anschaltmodul TAS10A040-N06-4X1-1, [3] Umrichter Drehtischmotor MDX60/61B 0075-5A3-4-00 [204], [4] Umrichter EtikettiermodulMotor MDX60/61B 00225-5A3-4-00 [204], [5] SPS, MOVI-PLC® advanced [195], [6] W-LAN Access-Point, [7] Not-Aus Schalter und Quittiertaster, [8] Hauptschalter, [9] Sicherungen, Leistungsschütze und Sicherheitsschaltgerät, [10] Netzfilter.

Baugruppen wie Frequenzumrichter, Gleichrichter, Anpass-Steller u.a. bestückt werden. Baugruppen mit hoher Wärmeverlustleistung sind direkt auf den Kühlkörper montiert. Der Kühlkörper fungiert gleichzeitig als Teil des Gehäuses, in Abbildung 3.11 an der Gehäuseunterseite erkennbar.

Durch den beschriebenen Aufbau ist ein lüfterloser Betrieb der Elektronikkomponenten möglich. Zur Verdrahtung der beweglichen Module stehen vorgefertigte Verdrahtungsplatinen und konfektionierte Leitungen zur Verfügung. Alle Anschlüsse zu den Elektronikteilen sind auf der Frontseite auf Steckvorrichtungen gelegt, so dass die Einheit, wenn notwendig, einfach und schnell ausgetauscht werden kann.

Die Abbildung 3.11 zeigt entsprechend dem Blockschaltbild Abbildung 3.10 die eingebauten Komponenten: Frequenzumrichter, Steuerung und Anpass-Steller. Ergänzende Baugruppen in dem Versuchsaufbau sind der WLAN-Client und das PLC-Modem 
Schnittstelle zum stationären Teil
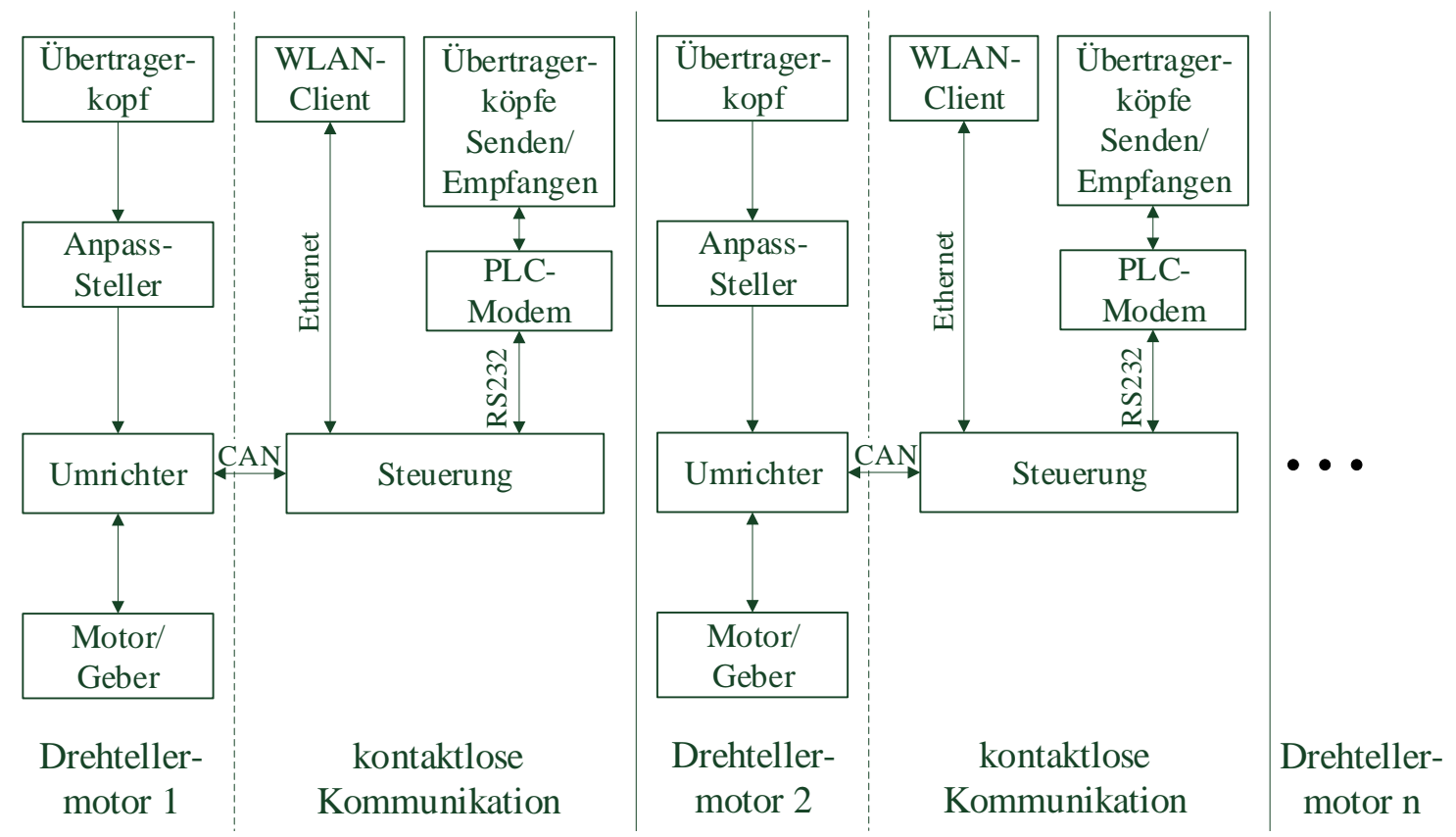

Abbildung 3.10.: Blockschaltbild der beweglichen elektrischen Komponenten.

für die Kommunikationsanbindung zum stationären Teil des Funktionsmusters. PLCModem und WLAN-Client ermöglichen die Ansteuerung der mobilen Komponenten und die Koordination der Prozessabläufe sowie den Austausch von Einstellparameter und Diagnoseinformationen.

Das PLC-Modem (vgl. Abbildung 4.9) ist im Bild noch nicht eingezeichnet. Das Modem wurde für den ersten Testbetrieb in einem eigenen Gehäuse eingebaut und verdrahtet. Neben einer Serviceschnittstelle ist zusätzlich noch ein Display für Statusanzeigen integriert. Die wesentlichen technischen Daten des Funktionsmusters sind in Abschnitt A beschrieben.

\section{5. Übertragungskanal der kontaktlosen Energieübertragung}

Der in Abschnitt 3.4 beschriebene Versuchsaufbau für die induktive kontaktlose Datenübertragung wurde hinsichtlich der Eigenschaften seines Übertragungsverhaltens untersucht. Bei der Datenübertragung über den Linienleiter zeigen sich auf dem Übertragungskanal verschiedenartige Störungseinflüsse, die die Kommunikation zwischen 


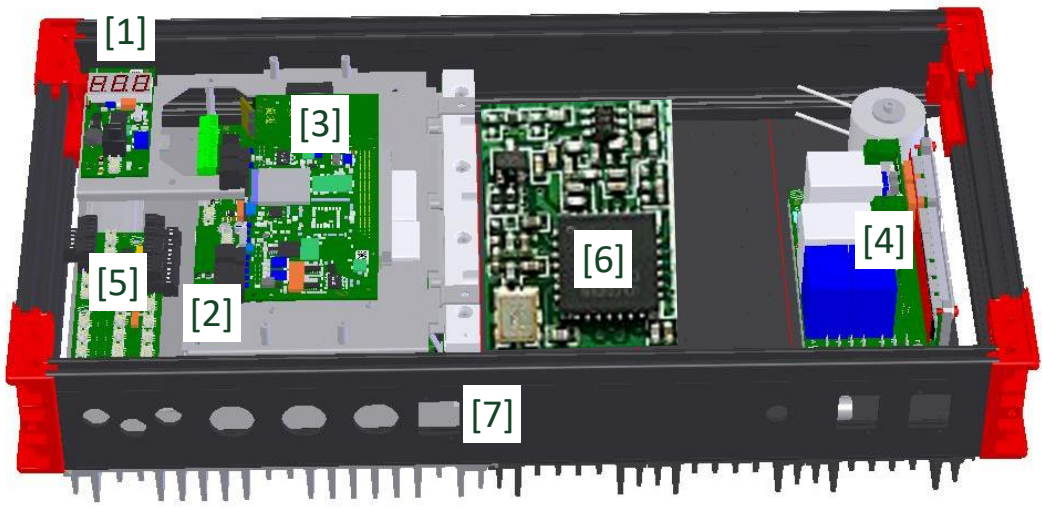

Abbildung 3.11.: Dezentrales Modul für bewegliche elektrische Verbraucher.

[1] Display, [2] Antriebsumrichter, [3] Steuerung, [4] Anpass-Steller,

[5] Verdrahtungsplatine, [6] WLAN-Client, [7] Einbaubereich Steckeranschlüsse.

den Teilnehmern negativ beeinflussen.

\subsubsection{Eigenschaften des Übertragungskanals}

Um die Eigenschaften des Übertragungskanals am Versuchsaufbau zu bestimmen, fungierte als Sender ein Funktionsgenerator vom Typ Agilent 33120A, der über einen Übertragerkopf die Signale kontaktlos über den Linienleiter zum Übertragerkopf des Empfängers überträgt. Auf der Empfangsseite wurden die Signale mit einem Digitaloszilloskop vom Typ LeCroy LT264 aufgezeichnet. Die Messungen zeigen das in Abbildung 3.12 skizzierte Übertragungsverhalten des Kanals. Das Übertragungsverhalten wird dabei entsprechend Glg. (3.3) definiert (vgl. [80]). s(f) beschreibt in Glg. (3.3) das frequenzabhängige Quellen- bzw. Empfangssignal. $H_{\text {Kanal }}(f)$ die Kanalübertragungsfunktion im Frequenzbereich.

$$
\left|H_{\text {Kanal }}(f)\right|=\left|\frac{\mathbf{s}_{\text {Empfänger }}(f)}{\mathbf{s}_{\text {Sender }}(f)}\right| .
$$

In Abbildung 3.12 zeigt sich, dass die Datenübertragung im Bereich von ca. 1, $5 \mathrm{MHz}$ .. 2, 2 M Hz die besten Übertragungseigenschaften aufweist. Außerhalb des Frequenzbereiches wird das Signal stark gedämpft. Im Frequenzbereich über $2 \mathrm{MHz}$ fällt zusätzlich die relative Permeabilität $\mu_{r}$ des Ferritmaterials des Übertragerkopfes stark ab und es treten dadurch hohe magnetische Verluste auf. 


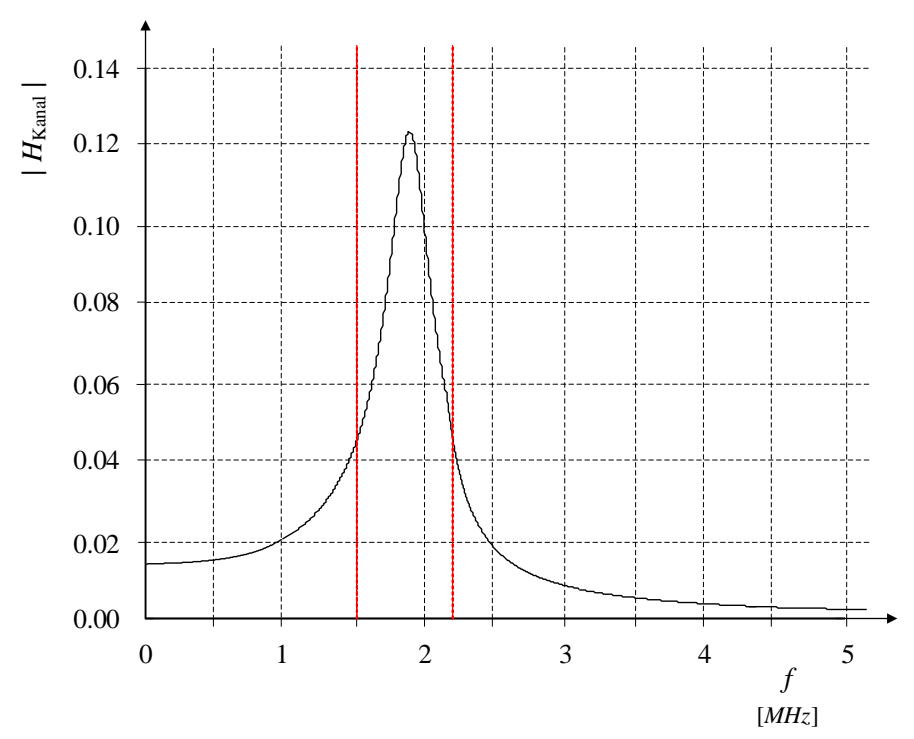

Abbildung 3.12.: Übertragungsverhalten des Datenkanals.

Die Ausbreitungsgeschwindigkeit elektromagnetischer Wellen im Vakuum, d.h. die Lichtgeschwindigkeit, wird per Definition mit $c_{0}=299792458 \mathrm{~m} / \mathrm{s}$ angegeben [117]. In anderen Medien verändert sich die Ausbreitungsgeschwindigkeit durch das verwendete Material. Die Ausbreitungsgeschwindigkeit im Medium berechnet sich gemäß Gl. (3.4) [117].

$$
c_{\text {medium }}=\frac{c_{0}}{\sqrt{\epsilon_{r} \mu_{r}}} .
$$

Die beiden Faktoren, relative Permittivität $\epsilon_{r}$ und relative Permeabilität $\mu_{r}$, sind beide frequenzabhängig. Die Ausbreitungsgeschwindigkeit der elektromagnetischen Welle in einem Kupferleiter bzw. der von elektrischen Signalen beträgt etwa $67 \%$ der Lichtgeschwindigkeit im Vakuum $\left(C_{\text {Kupfer }}=2 \cdot 10^{8} \mathrm{~m} / \mathrm{s}\right)$ [117]. Die theoretische durchschnittliche Signallaufzeit $t_{\text {Sig }}$ eines Signals, im englischen Sprachgebrauch als Propagation Delay bezeichnet, berechnet sich dann nach dem Weg-Zeit-Gesetz mit: $t_{\text {Sig }}=l_{K} / C_{\text {Kupfer }}$. Die Laufzeit des Signals im Kupferleiter beträgt somit pro Meter ca. 5 ns.

Zur Bestimmung der Signallaufzeiten am Funktionsmuster wurden Messungen mit einem Oszilloskop zwischen Master-Modem (Abfrage Ausgang Modem: Start Pilotsignal ) und Slave-Modem (Abfrage Eingang Modem: Pilotsignal erkannt) durchgeführt. Die Signallaufzeiten auf dem Linienleiter mit einer Länge von ca. $20 \mathrm{~m}$ betrugen ca. 100 ns (vgl. Abschnitt 4.3). 


\subsubsection{Störeinflüsse des Übertragungskanals}

Nach [238] lassen sich Störungen in einem $50 \mathrm{~Hz}$-Energieverteilungsnetz entsprechend ihrer Eigenschaften in fünf typischen Klassen einteilen:

- Schmalbandstörungen

- farbiges Rauschen

- periodische synchrone Impulsstörungen

- periodische asynchrone Impulsstörungen

- aperiodische asynchrone Impulsstörungen.

Die Störungen finden sich auch bei der Realisierung eines kontaktlosen Energieübertragungssystems (vgl. [80]). Schmalbandstörungen treten vor allem durch den eingeprägten $25 \mathrm{kHz}$-Linienleiterstrom und seinen Oberschwingungen auf. Die Ursache für die Schmalbandstörungen rührt vor allem vom Einspeise-Steller. Er wandelt die dreiphasige $50 \mathrm{~Hz}$ Eingangsspannung in eine einphasige Spannung von $25 \mathrm{kHz}$. Die dabei auftretenden Oberschwingungen treten wegen der vorhandenen Halbschwingungssymmetrie nur bei ungeraden Vielfachen auf, Gl. (3.5) [80], [104].

$$
f_{\text {Ober }}=f_{25 k H z}(2 n+1), \quad n=1,2,3, \ldots
$$

Der dem Einspeise-Steller nachgeschaltete Gyrator, dessen Schwingkreis auf $25 \mathrm{kHz}$ abgestimmt ist, schwächt zwar die Oberschwingungen ab, kann sie aber nicht komplett unterdrücken. Im Spektrum werden Grundschwingung und die nicht unterdrückten Oberschwingungen deshalb als Schmalbandstörungen sichtbar. Farbiges Hintergrundrauschen entsteht u.a. durch schnell getaktete digitale Schaltungen, meist in Verbindung von Überlagerungen von Störquellen kleiner Leistung.

Impulsstörungen sind generell Störsignale, die über einen kurzen Zeitraum eine hohe Amplitude aufweisen, das heißt, sie stören im Spektrum einen breiten Frequenzbereich. Periodische netzsynchrone Impulsstörungen entstehen bei der Erzeugung des $25 \mathrm{kHz}$-Stromes im Wechselrichter durch Schaltvorgänge der IGBTs (engl.: Insulated Gate Bipolar Transistor), sie sind synchron und periodisch zum $25 \mathrm{kHz}$ Energiestrom. Periodische und aperiodische netzasynchrone Impulsstörungen werden meist durch Ein- und Ausschaltvorgänge verursacht (vgl. [104]). 


\subsection{Zusammenfassung der Ergebnisse}

In Kapitel 3 wurde der Versuchsaufbau bzw. das Funktionsmuster einer Rundläufer-Etikettiermaschine vorgestellt. An dem Versuchsaufbau wurde gezeigt, wie ein induktives kontaktloses Energieübertragungssystem für eine rotative Prozessbewegung aufgebaut und in den Versuchsaufbau integriert ist. Die Abbildung 3.4 zeigt den realisierten Aufbau des Funktionsmusters.

Das Funktionsmuster findet Verwendung für die in Kapitel 4 durchzuführenden Untersuchungen betreffend der Datenübertragung und der Synchronisierung zwischen der stationären Basisstation und den beweglichen elektrischen Verbrauchern der sich drehenden Plattform. Zusätzlich wurde das Funktionsmuster in einem ersten Schritt betreffend des Datenaustausches zwischen stationärer und beweglicher Seite mit einer WLAN-Kommunikation ausgestattet, die für Start/Stopp-Vorgänge der Umrichter, Übergabe von Regelungs- und Einstellparameter sowie für Diagnosezwecke zur Verfügung stand.

Der Aufbau des Funktionsmusters lässt sich in zwei Hauptkomponenten einteilen. Einen feststehenden Teil, der die Trägerkonstruktion bildet und einen beweglichen sich drehenden Teil, der auf der Tragkonstruktion aufbaut. Im Versuchsaufbau dient der integrierte verlegte Linienleiter bzw. die Energieversorgungsstrecke gleichzeitig als Übertragungskanal für die Datenanbindung zu den einzelnen beweglichen Teilnehmern.

In Abschnitt 3.1 werden zuerst alle notwendigen funktionellen Komponenten des induktiven kontaktlosen Energieübertragungssystems detailliert beschrieben. Auf den Aufbau und die Funktionsabläufe einer realen Rundläufer-Etikettiermaschine wurde eingegangen, wie sie in der Getränkeindustrie zum Einsatz kommt. Im weiteren Verlauf des Abschnittes 3.4 wurden der Aufbau der verwendeten mechanischen und elektrischen Komponenten auf der stationären und beweglichen Seite des Funktionsmusters dargestellt und die Eigenschaften des Übertragungskanals erläutert. Das Übertragungsverhalten des Datenkanals über den Linienleiter wurde analysiert (vgl. Abbildung 3.12) und die Störeinflüsse des Übertragungskanals aufgezeigt.

Anhand der durchgeführten Untersuchungen am Funktionsmuster konnte gezeigt werden, dass die Datenübertragung im Bereich von 1, $5 \mathrm{MHz} \ldots 2,2 \mathrm{MHz}$ die beste Übertragungseigenschaft aufweist. Außerhalb des Frequenzbereiches wird das Signal stark gedämpft. Die Ergebnisse der Untersuchungen über die Qualität und die Eigenschaften des Datenkanals bilden den Ausgangspunkt für die in Kapitel 4 durchgeführten weiteren Arbeiten.

Im folgendem Kapitel 4 wird untersucht, inwieweit die beweglichen elektrischen Verbraucher des Funktionsmusters, die über das induktive kontaktlose Energieübertragungssystem mit Energie versorgt werden, miteinander datentechnisch synchronisiert 
werden können. Die Datenübertragung und Datensynchronisierung über den induktiven kontaktlosen Energieübertragungskanal werden dabei unter Einbindung möglicher Störszenarien wie Einschalt- und Ausschaltvorgänge der Maschine, Lastwechselspiele, Umweltbedingungen wie Temperatur, EMV und anderer Faktoren unter realen Bedingungen am Versuchsaufbau überprüft. 



\section{Datensynchronisierung über das kontaktlose Energieübertragungssystem}

Kapitel 4 geht der Frage nach, wie es möglich ist, bewegliche elektrische Verbraucher, die über das induktive kontaktlose Energieübertragungssystem mit Energie versorgt werden, miteinander datentechnisch zu synchronisieren. Ausgangspunkt für die Bewertung der Validierung der datentechnischen Synchronisierung bildet das in Kapitel 3 vorgestellte und aufgebaute Funktionsmuster einer Etikettiermaschine.

Bei der am Funktionsmuster aufgebauten induktiven kontaktlosen Energieübertragung zeigen sich verschiedenartige leitungsgebundene Störungen, welche die Datenübertragung negativ beeinflussen. Schmalbandstörungen werden verursacht durch den eingeprägten $25 \mathrm{kHz}$-Linienleiterstrom, periodische netzsynchrone Impulsstörungen durch Schaltvorgänge der IGBTs im Wechselrichter. Periodische und aperiodische netzasynchrone Impulsstörungen entstehen durch Ein- und Ausschaltvorgänge (vgl. Abschnitt 3.5.2).

Bedingt durch den stark gestörten Datenkanal und einer zu untersuchenden kontaktlosen Datenübertragung mit der Möglichkeit, die beweglichen elektrischen Verbraucher miteinander zu synchronisieren, wird daher ein besonders robustes Nachrichten-Übertragungsverfahren benötigt. Für den Austausch der einzelnen Datenpakete zwischen den Teilnehmern wird deshalb ein OFDM-Modulationverfahren (engl.: Orthogonal Frequency Division Multiplexing) gewählt [80].

Zunächst werden zu Beginn von Kapitel 4 die grundsätzlichen Anforderungen in Automatisierungssystemen hinsichtlich Synchronität und Gleichzeitigkeit aufgezeigt. Die Merkmale eines modernen Feldbussystems werden beschrieben. Die in der Arbeit eingebundenen digitalen Modulationsverfahren, das Referenzmodell des Kommunikationssystems und der Aufbau des Datenübertragungssystems werden vorgestellt. Der Aufbau des verwendeten PLC-Modems und seine wichtigsten Registerfunktionen für die Datenübertragung werden erläutert.

Im weiteren Verlauf des Kapitels werden die Umsetzung des Master-Slave-Betriebs und die Einbindung der SSC-Schnittstelle beschrieben sowie die Parameter des Da- 
tenübertragungssystems bestimmt. Im Abschnitt 4.4 wird im Detail die Realisierung vorgestellt, wie bewegliche elektrische Verbraucher über den induktiven kontaktlosen Energieübertragungskanal miteinander synchronisiert werden.

Dabei wird gezeigt, dass durch die Verwendung eines Zeitstempelverfahrens eine effektive Synchronisierung zwischen den beweglichen elektrischen Teilnehmern möglich ist. Im ersten Schritt wird die Möglichkeit betrachtet, das Datentelegramm um das Zeitstempelverfahren zu erweitern. Im weiteren wird untersucht, inwieweit die Verzögerungen bzw. die Durchlaufzeiten im Sende- und Empfangspfad des Modems so bestimmt werden können, dass eine Synchronisierung zwischen den beweglichen elektrischen Verbrauchern möglich ist. Dabei werden die Durchlaufzeiten innerhalb der OFDM-basierten Datenkommunikation der Teilnehmer durch parallele Abarbeitungsstrukturen ermittelt.

Im letzten Abschnitt von Kapitel 4 wird der Einfluss der Gangabweichung von Oszillatoren untersucht und beurteilt. Im Anhang in Abschnitt B sind die Wirkungsprinzipien von Oszillatoren und die Eigenschaften von Schwingquarzen zum grundlegenden Verständnis dargestellt. Auf die unterschiedlichen Quarzschnitte von Schwingquarzen, ihre Temperaturabhängigkeiten, ihre Alterungseffekte sowie auf die Definition der Kurzzeitstabilität und die Allan-Varianz wird eingegangen. Die Allan-Varianz beschreibt dabei ein Maß für die Stabilität von Oszillatoren.

Im Abschnitt 4.5 wird dann konkret untersucht, welche Ganggenauigkeiten bei verschiedenen Oszillatorklassen erreicht werden und inwieweit sich durch den Einsatz von höherwertigen Oszillatoren die Taktfrequenz für die Synchronisierungsvorgänge bei der Übertragung von Daten zwischen den einzelnen Kommunikationsteilnehmern reduzieren lässt. In einem praktischen Versuchsaufbau werden die Ganggenauigkeiten von Oszillatoren unterschiedlicher Oszillatorklassen durch die Zwei-OszillatorenMethode qualitativ bewertet.

Im folgendem Abschnitt sollen zunächst die grundsätzlichen Anforderungen an $\mathrm{Au}$ tomatisierungssysteme bei der Übertragung von Prozessdaten betrachtet werden. Dabei spielen die Begriffe Synchronität und Gleichzeitigkeit eine zentrale Rolle.

\subsection{Synchronität und Gleichzeitigkeit}

Die klassische SPS-Technik (Speicherprogrammierbare Steuerung) findet in der heutigen Zeit in vielen unterschiedlichen Applikationen in der Fabrik- und Prozessautomation Verwendung. Dabei definieren der Umfang und die Geschwindigkeit des Prozesses die notwendige Reaktionsfähigkeit der Steuerung. Die wesentlichen Kriterien bei der Auswahl der Steuerung für die entsprechende Anlage oder Maschine 
sind die Anzahl der vorhandenen I/O-Punkte (engl.: Input/Output ${ }^{1}$ ), andere analoge oder nicht binäre zu verarbeitende Prozessgrößen sowie die Verriegelungstiefe und die Verflechtung der Prozessgrößen zueinander.

Anhand der vorliegenden Randbedingungen ist ein Prozessor bzw. eine CPU-Baugruppe (engl.: Central Prozessing Unit) zu wählen, die die Leistungsmerkmale hinsichtlich Prozessorleistung und Größe des Programmspeichers erfüllt. Typische Programmabarbeitungszeiten bzw. die Zykluszeit für das Einlesen der Eingänge bis zum Schreiben der Ausgänge liegen meist im Bereich von 10 ...20 ms.

Sollen hochdynamische und präzise Bewegungen über Automatisierungssysteme oder dezentrale Antriebslösungen aufeinander abgestimmt werden, sind nicht nur eine kurze Zykluszeit, sondern auch eine hohe Gleichzeitigkeit und eine hohe Synchronität (kleiner Jitter) beim Übertragen der Prozessdaten gefordert. Typische Anwendungen sind z.B. Gleichlaufantriebe oder elektronische Kurvenscheiben [52], [101]. Abbildung 4.1 verdeutlicht den Zusammenhang von Gleichzeitigkeit und Synchronität (vgl. [89]). Auch wenn Synchronität zwischen zwei Teilnehmern gegeben ist, kann trotzdem die Gleichzeitigkeit bzw. der Startpunkt, der ein Ereignis auslöst, davon abweichen.

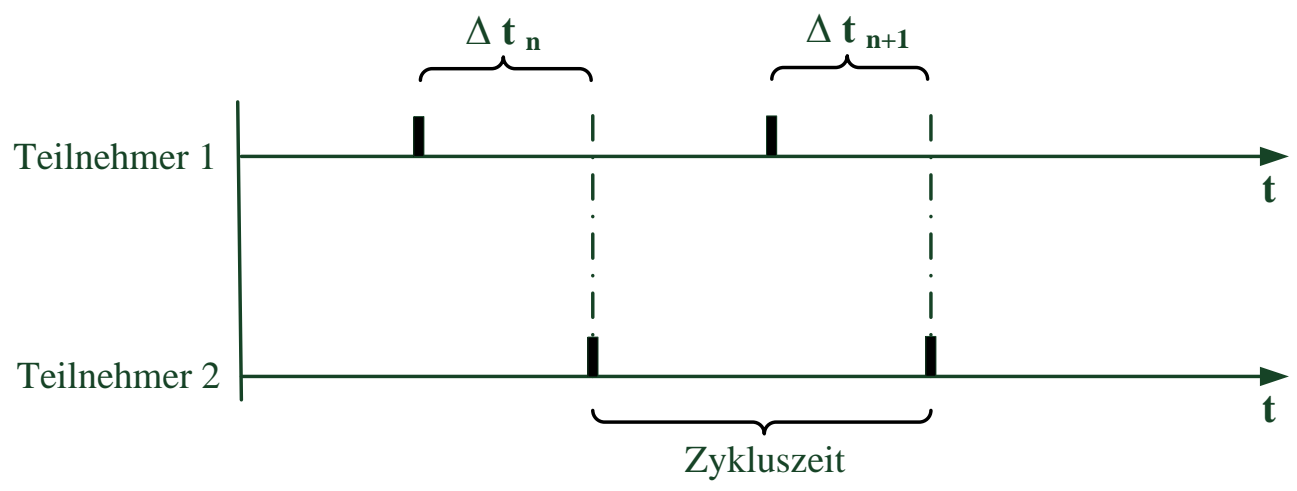

$$
\begin{aligned}
\text { Gleichzeitigkeit } & =\left|\Delta t_{n}\right| . \\
\text { Synchronität (Jitter) } & =\left|\Delta t_{n+1}-\Delta t_{n}\right| .
\end{aligned}
$$

Abbildung 4.1.: Gleichzeitigkeit und Synchronität [89].

Um Synchronisierungsvorgänge in Automatisierungssystemen zu realisieren, können unterschiedliche Ansätze verfolgt werden. Eine einfache und klassische Variante,

\footnotetext{
${ }^{1}$ binäre Eingangs- und Ausgangssignale zu bzw. von einer Steuerung
} 
Teilnehmer in einem Netzwerk zu synchronisieren, ist Synchronisationssignale oder Synchronisations-Telegramme in einem festen Zeitraster mit möglichst geringer Abweichung im Netzwerk zu versenden. Das Synchronisations-Telegramm kann als Uni-, Multi- oder Broadcast-Telegramm aufgebaut sein. Oft basiert die Kommunikation dabei auf einem Zeitschlitzverfahren auf Basis einer Master-Slave-Architektur. Jeder Empfänger synchronisiert seine Prozessaufgaben, in der Regel auf den aktuellen Empfangszeitpunkt. Steht kein spezielles Synchronisations-Telegramm zur Verfügung, kann der Synchronisationszeitpunkt auch aus dem abgeleiteten Signal aus dem zyklischen Datentelegramm bzw. dem Protokoll bestimmt werden.

Um eine möglichst hohe Gleichzeitigkeit in einem verteilten System zu gewährleisten, sollten alle Signallaufzeiten zwischen den Teilnehmern bekannt sein. Bearbeitungszeiten und Verzögerungszeiten, die beim Durchlauf durch die Busknoten oder anderer Komponenten entstehen, müssen gleichermaßen berücksichtigt werden. In einem solchem Fall können alle Teilnehmer in einem verteilten System auf einer gemeinsamen Zeitbasis arbeiten. Das Prinzip machen sich auch einige Feldbussysteme zunutze [69], [105], [171]. Auf Basis verteilter Uhren gemäß IEEE-1588 (engl.: Institute of Electrical and Electronics Engineers) und durch Synchronisierung mittels entsprechender Telegramme wird eine gemeinsame präzise Zeitbasis generiert. Bei der Methode auf Basis verteilter Uhren ist es auch denkbar und möglich, Prozessdaten mit einem entsprechenden Vorlauf zu übertragen, d.h. zum Synchronisationszeitpunkt stehen die Daten für die Verarbeitung beim Teilnehmer bereits zur Verfügung.

\subsubsection{IEEE-1588 - Precision Time Protocol}

IEEE-1588 „Standard for a Precision Clock Synchronization Protocol for Networked Measurement and Control Systems" oder auch kurz PTP (engl.: Precision Time Protocol) definiert ein Protokoll zur präzisen Synchronisation lokaler Echtzeituhren in verteilten Systemen (vgl. [53]).

Ein PTP-System besteht aus einer unterschiedlichen Anzahl von Netzwerkknoten, die über ein paketvermittelndes Netzwerk miteinander verbunden sind. Jeder Knoten bildet dabei auch eine dezentrale Uhr. Das Protokoll eignet sich sehr gut für die Integration in Ethernetnetzwerken oder generell in paketvermittelnden Protokollen, die Multicast-Adressen unterstützen. Dabei ist es möglich, die verteilten Uhren in den Knoten im Mikrosekundenbereich durch ein Zeitstempelverfahren auf eine Masteruhr zu synchronisieren.

Bei PTP in Hardware-Ausführung sind Synchronisationsgenauigkeiten im Bereich von Nanosekunden möglich. Im einfachsten Fall besteht das Netzwerk aus einer Master Clock und mehreren Slave Clocks. Wird das System durch weitere Knoten erweitert oder der aktuelle Knoten mit der Master Clock vom System getrennt, wird über 
den sogenannten BMC-Algorithmus (engl.: Best Master Clock) die genaueste Uhr bestimmt und eine neue Master Clock dem Netzwerk zugewiesen.

Um zwei oder mehrere verteilte Uhren aufeinander zu synchronisieren, sind zwei Größen zu betrachten bzw. zu bewerten. Zum einen die relative Zeitdifferenz zwischen der Masteruhr und der einzelnen Slaveuhr selbst, im englischen Sprachgebrauch als Offset bezeichnet, und zum anderen die Verzögerungszeit, die beim Übertragen des Synchronisationssignales bzw. des Zeitstempels entsteht. Die Verzögerungszeit, englisch Delay, wird verursacht durch die reine Laufzeit des Signals auf dem Übertragungskanal. Auch Signalverzögerungen in den Netzwerkkomponenten, wie z.B. in Repeater, Switches oder Router, tragen dazu bei. Im Protokoll gemäß IEEE-1588 sind insgesamt vier Telegrammarten definiert.

Telegrammarten:

- Synchronisationstelegramm (Sync)

- Nachfolgetelegramm (Follow-Up)

- Verzögerungszeitanfrage (Delay-Request)

- Antwort auf die Verzögerungszeitanfrage (Delay-Response).

Abbildung 4.2 verdeutlicht den generellen Ablauf des Synchronisationsmechanismus. Der Master sendet periodisch per Multicast seinen aktuellen Zeitstempel mittels Sync-Telegramm an alle Slave (Zeitpunkt $t_{1}$ ). Der übertragene Zeitstempel weicht allerdings vom tatsächlichen Sendezeitpunkt der Masteruhr ab, da Laufzeiten und Verzögerungszeiten in der Masteruhr bis zum Versenden der Nachricht nicht berücksichtigt werden konnten. Aus dem Grund wird die Verzögerungszeit im Master (Zeitstempel im Sync-Telegramm hinterlegen bis zum exakten Zeitpunkt des Versendens des Sync-Telegramms) gemessen und in einem Nachfolgetelegramm (Follow-Up) nachgesendet.

Durch den hinterlegten Zeitstempel im Sync-Telegramm und durch den Korrekturwert im Follow-Up-Telegramm kann somit jede Slaveuhr ihren Offset zur Masteruhr berechnen und korrigieren (Zeitpunkt $t_{2}$ ). Allerdings sind zu dem Zeitpunkt Fehler aus der Übertragungszeitdauer des Netzwerkes noch nicht berücksichtigt. Um die Übertragungszeit zwischen Master und Slave zu bestimmen, sendet der Slave nach dem Erhalt der Sync-Message ein Delay-Request-Telegramm mit seiner exakten Sendezeit (Zeitpunkt $t_{3}$ ).

Die Sendezeit wird gleichzeitig auch im Slave zwischengespeichert. Der Master speichert die genaue Empfangszeit des Delay-Request-Telegramms (Zeitpunkt $t_{4}$ ) und schickt den Wert im Delay-Response-Telegramm an die zugehörige Slaveuhr zurück. 

Master-
Slave-
Clock
Clock

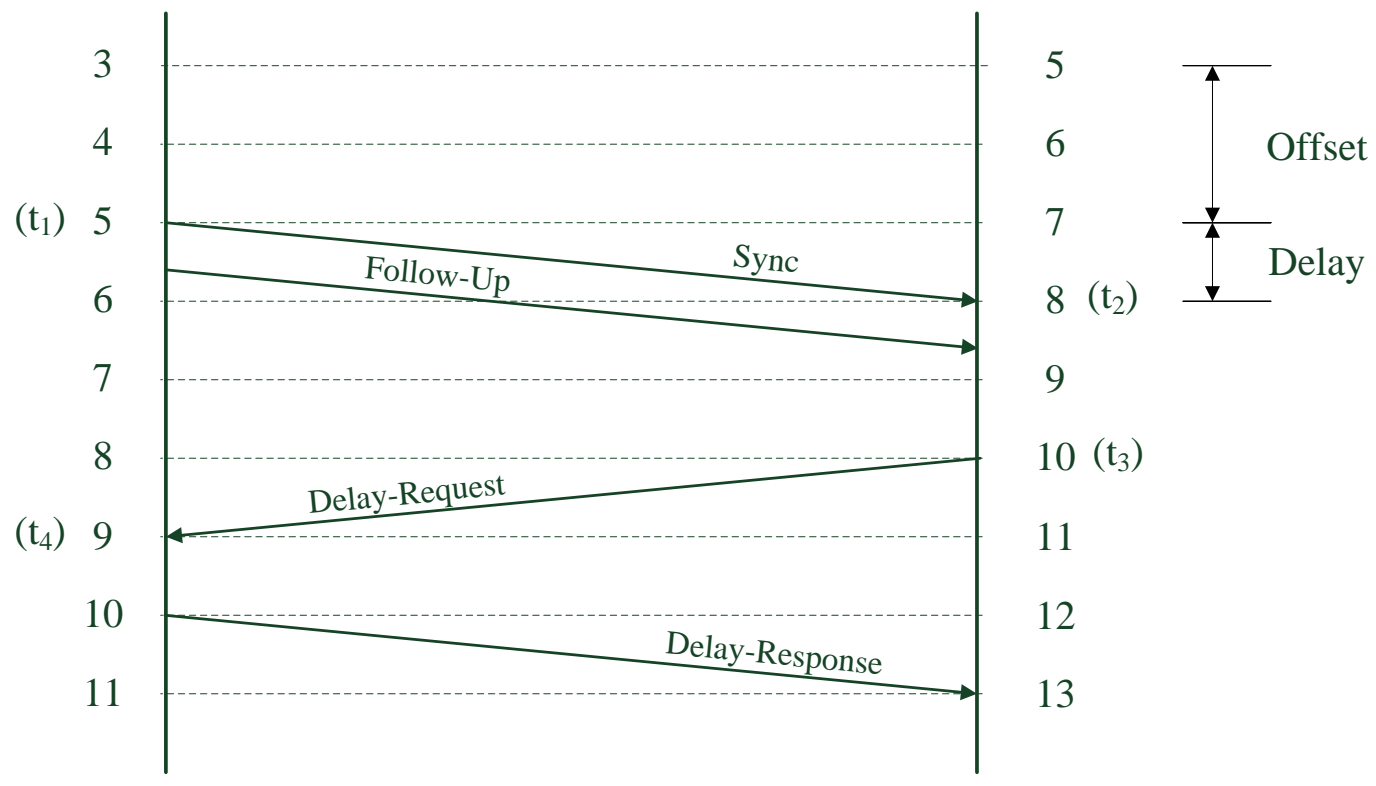

Abbildung 4.2.: IEEE-1588 Synchronisationstelegramme [53].

Entsprechend Gl. (4.3) und Gl. (4.4) können durch die bestimmten Sende- und Empfangszeitpunkte $\left(t_{1}, t_{2}, t_{3}, t_{4}\right)$ Offset und Delay zur exakten Uhrensynchronisation zwischen Master- und Slaveuhr berechnet werden. Gl. (4.5) und Gl. (4.6) folgen durch einfache Addition bzw. Subtraktion der Gl. (4.3) und Gl. (4.4). Anzumerken ist bei dem Verfahren, dass bei der Berechnung der Übertragungszeiten von gleichen Laufzeitverhältnissen des Übertragungskanals bei der Signalübertragung zwischen Master und Slave und zwischen Slave und Master auszugehen ist.

$$
\begin{aligned}
& t_{2}=t_{1}+\text { Delay }+ \text { Offset. } \\
& t_{4}=t_{3}+\text { Delay }- \text { Offset. }
\end{aligned}
$$

Mit $\Delta t_{1}=t_{2}-t_{1}$ und $\Delta t_{2}=t_{4}-t_{3}$ folgt:

$$
\begin{aligned}
\text { Delay } & =\frac{\Delta t_{1}+\Delta t_{2}}{2} . \\
\text { Offset } & =\frac{\Delta t_{1}-\Delta t_{2}}{2} .
\end{aligned}
$$




\subsubsection{Moderne Feldbussysteme}

Feldbussysteme finden heute Verwendung in fast allen Bereichen der Fertigungs-, Prozess- und Gebäudeautomatisierung [105], [135], [185], [187]. Vor Einführung der Feldbussysteme in den 1980er Jahren war es üblich, Sensoren und Aktoren von Maschinen oder Maschinenteilen durch eine aufwendige Verdrahtung an eine zentrale Steuerung über I/O-Module und Klemmen im Schaltschrank anzuschließen. Der Schaltraum befand sich in der Regel in einem, wenn notwendig, klimatisierten Schaltraum. Sensor- und Aktoranschlussleitungen wurden zunächst auf einen Klemmkasten, der direkt am Maschinenteil montiert war, angeschlossen. Anschließend wurden Verbindungsleitungen vom Klemmenkasten zum Schaltschrank verlegt.

Das führte vor allem bei großen Anlagen mit einem hohen Automatisierungsgrad zu einem erheblichen Installationsaufwand. Die Montage von Kabelpritschen über Flur, Einlassen von Kabelrinnen oder Kabelrohren in den Hallenboden waren planungsund kostenintensiv und verzögerten den Aufbau der Maschinen und der Anlagenteile. Auch das Verlegen der Kabel selbst über lange Strecken zum Schaltschrank war aufwendig und durch die gebündelte Verlegung der Kabel neben- und übereinander störanfällig. Um die Störsicherheit zu verbessern, wurden abgeschirmte Leitungen verwendet. Sensor- und Aktorleitungen wurden nach Möglichkeit getrennt oder mit seitlichem Abstand zueinander verlegt.

Bei Einführung der Feldbussysteme lag der Fokus vor allem darin, die parallele Leitungsverdrahtung der Sensoren, d.h. die digitale Eingangsebene, durch eine einzige Busleitung zu ersetzen. Auf spezielle Leitungen, wie sie z.B. für die Übertragung bei Analogsignalen benötigt wurden, konnte durch die Digitalisierung bzw. durch die Analog-Digital-Wandlung (A/D) in den Busmodulen vor Ort verzichtet werden gleichzeitig wurde dadurch die Störsicherheit verbessert. Heute sind und werden auch immer mehr Aktoren, wie Frequenzumrichter, auch in größeren Leistungsbereichen, Ventile usw. dezentralisiert. Die Verkabelung zwischen Schaltschrank und dezentraler Peripherie reduziert sich letztlich auf die Verlegung der Busleitung und die eines Leistungskabels zur Energieversorgung der Aktoren.

Die Anforderungen an ein modernes Feldbussystem sind vielfältig. Neben dem beschriebenen geringeren Installationsaufwand soll das System leicht verständlich, einfach und flexibel konfigurierbar sein. Wartungsaufwand und Ersatzteilhaltung sollten gering sein, die Fehlerbehebungszeiten möglichst klein. Dabei sollen kurze Zykluszeiten durch hohe Übertragungsgeschwindigkeiten realisiert werden. Bei dynamischen Anwendungen werden zusätzlich Forderungen hinsichtlich Gleichzeitigkeit und Synchronität, d.h. geringem Jitter, an das Feldbussystem gestellt.

Moderne Bussysteme sollen auch immer mehr die Funktionalität unterschiedlicher spezialisierter Bussysteme, wie z.B. I/O-, Antriebs- und Kommunikationsbusse, ver- 
einen. Dadurch ist eine durchgehende Kommunikation innerhalb der Automatisierungspyramide, ausgehend von der ERP-Ebene (engl.: Enterprise Resource Planning) über die Prozessleitebene bis zur Feldebene im industriellen Umfeld möglich. Am Markt haben sich mittlerweile eine Vielzahl von Feldbussystemen etabliert. Dabei bestimmt in der Regel der Typ der Kopfsteuerung (meist SPS oder Industrie-PC eines Herstellers) bzw. das dort zum Betrieb notwendige Bus-Anschaltmodul, welcher Feldbus zum Einsatz kommt.

Profibus, DeviceNet, Interbus, CANopen, Sercos sind nur einige von vielen Feldbusanbietern [105]. Die zweite Generation der Feldbustechnik, wie EtherCAT, Sercos III, EtherNet/IP, ProfiNet (RT, IRT), Powerlink, Modbus/TCP - auch hier seien nur einige erwähnt - basieren auf der Echtzeit-Ethernet-Technologie. Feldbusse werden seit 1999 in der IEC-Norm (engl.: International Electrotechnical Commission) 61158: „Fieldbus for use in industrial control systems“ und einzelne Feldbusse in der IECNorm 61784-1: „Communication Profile Families (CPF)“ international standardisiert. Ethernet-basierte Feldbusse sind in der Norm IEC 61784-2 aufgeführt - die Protokollspezifikationen in der Norm IEC 61158. Bei den Bussystemen wird unterscheiden zwischen [185]:

- teilnehmerorientierten Bussystemen und

- nachrichtenorientierten Bussystemen.

Teilnehmerorientierte Bussysteme sind z.B. Ethernet und Profibus. CAN-basierte Bussysteme arbeiten nach einem nachrichtenorientierten Prinzip. Eine weitere Unterscheidung kann hinsichtlich des Buszugriffsverfahrens getroffen werden:

- zufälliger Buszugriff

- Master-Slave-Prinzip

- Token-Prinzip.

Beim zufälligen Buszugriffsverfahren (CSMA, engl.: Carrier Sense Multiple Access) prüft jeder Busteilnehmer, ob der Kommunikationskanal frei ist von Übertragungsvorgängen. Schreiben zwei oder mehrere Teilnehmer gleichzeitig auf den Bus, was bedingt durch die Signallaufzeiten auftreten kann, kommt es zur Datenkollision, was aber per Prinzip zugelassen ist. Die Kollision wird erkannt und es erfolgt eine weitere Nachricht zu einem späteren Zeitpunkt.

Bei Verwendung des Master-Slave-Zugriffsverfahrens koordiniert der Master zentral das Buszugriffsrecht. Die Kommunikation erfolgt ausschließlich zwischen Master und den einzelnen Slaves. Der Ablauf bzw. der Zugriff zu den einzelnen Slaves wird in der Regel über ein Zeitschlitzverfahren gesteuert. 
Beim Token-Prinzip erfolgt ein kontrollierter dezentraler Buszugriff. Ein expliziter Master ist nicht vorhanden. Das Buszugriffsrecht, in Form des Tokens, wird zyklisch weitergereicht.

Alle Feldbussysteme erfüllen die Aufgabe, dezentrale Ein- und Ausgangsmodule bzw. deren Daten zyklisch einzulesen und nach der Bearbeitung durch die Steuerung wieder auszugeben. Trotzdem unterscheiden sie sich in wesentlichen Dingen wie der Art des Übertragungsmediums, mögliche maximale Leitungslängen und maximal zu übertragenden Datenraten sowie durch den topologischen Aufbau wie Linie, Stern, Baum, Ring oder deren Mischstrukturen. Auch Übertragungsverfahren, die Anschlusstechnik und die maximale Anzahl der Busteilnehmer variieren stark innerhalb der angebotenen technischen Systeme. Unterscheidungsmerkmale sind auch, inwieweit azyklisch Daten zwischen den Teilnehmern verschickt werden können und ob ein Querverkehr zwischen den Busknoten möglich ist. Ein wichtiger Punkt ist, inwieweit eine exakte zeitliche Synchronisation zwischen den Feldbus-Teilnehmern möglich ist. Bei einigen Echtzeit-Ethernet-Protokollen werden verteilte Uhren dazu verwendet, um die bei der zyklischen Übertragung auftretenden Jitter zu verringern.

\subsection{Schichtenmodell und Modulation}

Soll eine Nachricht von einem Sender zu einem Empfänger übertragen werden, durchlaufen die Nachrichten in der Regel unterschiedliche Schichten innerhalb eines Kommunikationssystems. Um Kommunikationsabläufe zu standardisieren und eine Weiterentwicklung über unterschiedliche Systeme hinweg zu ermöglichen, wurde von der Internationalen Organisation für Normung - kurz ISO $^{2}$ das sogenannte OSISchichtenmodell (engl.: Open Systems Interconnection Model) eingeführt.

Abhängig von der Art der Übertragungsstrecke zwischen Sender und Empfänger muss das Nachrichtensignal an die Strecke angepasst werden. Dem Nachrichtensignal wird dabei ein Trägersignal aufgeprägt. Der Träger kann dabei z.B. sinusförmig sein (Modulation außerhalb vom Basisband) oder pulsförmig (Modulation im Basisband). Wird der sinusförmige Träger mit einem kontinuierlichen Nachrichtensignal moduliert, spricht man von der der klassischen Modulation oder analogen Modulation, ein Standardverfahren in der analogen Rundfunk- und Fernsehübertragung.

\subsubsection{Referenzmodell des Kommunikationssystems}

Das Referenzmodell für den Aufbau eines herstellerunabhängigen Kommunikationssystems kann als OSI-Schichtenmodell dargestellt werden. Es besteht aus sieben

\footnotetext{
${ }^{2}$ aus dem griechischen isos: gleich
} 
Schichten (engl.: layers). Abbildung 4.3 zeigt den Aufbau der einzelnen Schichten. Das aufgezeigte Modell wie auch andere ISO-Standards stellen dabei eine Empfehlung dar.

\begin{tabular}{|c|c|c|c|}
\hline Schicht: & Teilnehmer 1 & Teilnehmer 2 & \multirow{4}{*}{$\begin{array}{l}\text { anwender- } \\
\text { orientierte } \\
\text { Schichten }\end{array}$} \\
\hline 7 & $\begin{array}{l}\text { Anwenderschicht/ } \\
\text { Application Layer }\end{array}$ & Anwenderschicht & \\
\hline 6 & $\begin{array}{l}\text { Darstellungsschicht/ } \\
\text { Presentation Layer }\end{array}$ & Darstellungsschicht & \\
\hline 5 & $\begin{array}{l}\text { Sitzungsschicht/ } \\
\text { Session Layer }\end{array}$ & Sitzungsschicht & \\
\hline 4 & $\begin{array}{l}\text { Transportschicht/ } \\
\text { Transport Layer }\end{array}$ & Transportschicht & \multirow{4}{*}{$\begin{array}{l}\text { transport- } \\
\text { orientierte } \\
\text { Schichten }\end{array}$} \\
\hline 3 & $\begin{array}{l}\text { Vermittlungsschicht/ } \\
\text { Network Layer }\end{array}$ & Vermittlungsschicht & \\
\hline 2 & $\begin{array}{c}\text { Sicherungsschicht/ } \\
\text { Data Link (Mac)-Layer }\end{array}$ & Sicherungsschicht & \\
\hline 1 & $\begin{array}{l}\text { Bitübertragungsschicht/ } \\
\text { Physical (PHY)-Layer }\end{array}$ & Bitübertragungsschicht & \\
\hline
\end{tabular}

Abbildung 4.3.: OSI-Schichtenmodell [55].

Jeder Schicht werden bestimmte Funktionen und Protokolle und jeweils eng begrenzte Aufgaben zugeordnet. Allerdings kommt es in der Praxis oft vor, auch aus historischen Gründen beim Aufbau eines Kommunikationssystems, dass die Grenzen zwischen den unterschiedlichen Schichten miteinander verschmelzen oder gar nicht nach der Definition des Schichtenmodells aufgebaut wurden. Die Schichten 1...4 zählen zu den sogenannten transportorientierten Schichten, die Schichten $5 \ldots 7 \mathrm{zu}$ den anwendungsorientierten Schichten. Schicht 7 bildet die oberste Schicht im OSIModell.

Neben der Dateneingabe und Datenausgabe stellt die Anwenderschicht die Funktionen für die eigentliche Anwendung zur Verfügung. In der Darstellungsschicht werden die systemabhängigen Daten von und zur Anwendungsschicht in ein unabhängiges Standard-Format gewandelt. Weitere Aufgaben sind die Datenkompression und Verschlüsselungstechnik.

Die Sitzungsschicht oder auch Kommunikationsschicht steuert die logische Verbindung zwischen den Teilnehmern, d.h. organisiert und synchronisiert die Verbindung 
und den Datenaustausch. Schicht 4 verbindet die transportorientierten und anwendungsorientierten Schichten, dadurch wird Schicht 5 bis 7 ein einheitlicher Zugriff ermöglicht. Die Datenpakete werden dabei einer konkreten Anwendung zugeordnet. Schicht 3 steuert die Kommunikation, d.h. sorgt für das Schalten der Verbindungen bzw. bei Datenpaketen für die Weitervermittlung der Daten zum nächsten Knoten oder Empfänger. Die Schicht 2 regelt den Zugriff auf das Übertragungsmedium und die physikalische Adressierung der Datenpakete. Der Bitdatenstrom wird in Frames segmentiert. Zur Vermeidung von Übertragungsfehlern werden funktionale Fehlererkennungsmechanismen, z.B. Prüfsummen, eingebunden.

In Schicht 1 wird schließlich die physikalische Übertragung der Information über den Übertragungskanal definiert. Sie bildet die Schnittstelle zum Übertragungsmedium, wobei das Übertragungsmedium selbst kein Bestandteil der Schicht ist. Die Bitfolge wird dabei in ein zum Übertragungsmedium passendes Signal umgewandelt. Das können in der Nachrichtentechnik z.B. elektrische und optische Signale sein oder auch elektromagnetische Wellen bei drahtlosen Netzwerken.

Im Powerline-Modem entsprechend Abschnitt 4.3.1 ist die Bitübertragungsschicht: Schicht 1, PHY-Layer (engl.: PHYsical) und die Sicherungsschicht: Schicht 2, MACLayer (engl.: Media Access Control), innerhalb der FPGA-Umgebung implementiert, die anwendungsorientierten Schichten im Mikroprozessor. Die Bitübertragungsschicht hat die Aufgabe der bitseriellen Übertragung von Datensignalen durch OFDMSymbole. Die Sicherungsschicht übernimmt Aufgaben der Fehlererkennung und Fehlerbehebung bei der Übertragung der Datenpakete.

\subsubsection{Digitale Modulationsverfahren}

Digitale Modulationsverfahren außerhalb des Basisbandes beschreiben die Umformung bzw. die Zuweisung zeit- und wertdiskreter Signale, also digitaler Werte, in ein zeitkontinuierliches Sende- bzw. Trägersignal. Ein oder mehrere Bit werden dem analogen Signalzustand zugeordnet, über den Übertragungskanal übertragen und im Empfänger durch den Demodulator wieder zurück in die ursprüngliche Bitfolge rekonstruiert. Im Bereich der Nachrichtentechnik kann bei der Klassifizierung unterschiedlicher Modulationsverfahren dabei zwischen schmal- und breitbandigen Übertragungsverfahren unterschieden werden.

Bei einer Funkübertragung bzw. bei der kontaktlosen Nachrichtenübertragung über den Linienleiter wird das Nachrichtensignal im Basisbandbereich auf die Trägerfrequenz moduliert. Jedes auf dem Übertragungskanal aufgebrachte modulierte Sendesignal lässt sich dabei in der Form von Gl. (4.7) darstellen [92].

$$
s(t)=a(t) \cos \left(2 \pi f_{T} t+2 \pi f_{I} t+\theta(t)\right) .
$$


In Gl. (4.7) lassen sich drei Parameter verändern, um Informationen auf die Trägerfrequenz $f_{T}$ aufzubringen. Im Einzelnen sind das die Amplitude $a(t)$, die Informationsfrequenz $f_{I}$ und die Phasenlage $\theta(t)$. Eine weitere sehr einfache Möglichkeit, ein binäres Signal zu modulieren, ist die sogenannte Ein/Aus-Umtastung (OOK, engl.: On/Off Keying). Je nach 0- oder 1-Zustand der zu sendenden Bitfolge wird die Trägerfrequenz entsprechend ein- oder ausgeschaltet. Nachteile sind die hohe Störanfälligkeit bei hoher Dämpfung auf der Übertragungsstrecke und die geringe Datenrate.

Wird die Signalamplitude $a(t)$ variiert, spricht man von der Amplitudenumtastung oder ASK (engl.: Amplitude Shift Keying). Bei ASK findet eine Umtastung zwischen zwei (2-ASK oder binärer ASK) oder mehreren Abstufungen des Amplitudensignals statt. Bei Verwendung mehrerer Amplitudenstufen lassen sich pro Amplitudenstufe mehrere Bit zuordnen.

Die Veränderung der Informationsfrequenz $f_{I}$ führt zur Frequenzumtastung bzw. FSK (engl.: Frequency Shift Keying). Bei der binären FSK wird zwischen zwei Sendefrequenzen umgeschaltet, je nach Zustand des binären Nachrichtensignals. Bei MFSK steht $M$ für die Anzahl der verwendeten unterschiedlichen Frequenzen. Wird die Phasenlage $\theta(t)$ des Trägers variiert, spricht man von Phasenumtastung oder PSK (engl.: Phase Shift Keying). QPSK (engl.: Quadrature Phase Shift Keying) definiert z.B. das Umschalten zwischen vier um jeweils um $90^{\circ}$ versetzten Phasenlagen. Das ermöglicht die Übertragung von 2 Bit pro Sendesymbol. Eine Kombination aus ASK und PSK führt zu der Quadraturamplitudenmodulation (QAM), im englisch als QASK (Quadrature Amplitude Shift Keying) bezeichnet. In Abbildung 4.4 ist die Konstellation der Signalzustände für eine 16-QAM abgebildet. Insgesamt können zwei Amplituden vier Phasenwerte und einer Amplitude acht Phasenwerte zugeordnet werden. Die 16 unterschiedlichen Phasenlagen bzw. Amplituden ermöglichen es, jeder Zeigerstellung 4 Bit zuzuordnen.

Der Abstand der einzelnen Signalpunkte in der I-Q-Ebene zueinander hat direkten Einfluss auf den Störabstand bzw. die mögliche Übertragungsqualität des Signales. Bei 16-PSK z.B. liegen alle Signalpunkte auf einem Kreis enger beieinander, bei 16-QASK sind sie in der I-Q-Ebene besser verteilt [156]. Bei einer Einzelträgerübertragung werden die zuvor beschriebenen unterschiedlichen Modulationsverfahren verwendet, um einzelne Signale auf die zugehörige Trägerfrequenz zu modulieren. Multiplexverfahren sind Verfahren, in denen gleichzeitig mehrere Signale über den Übertragungskanal übertragen werden. Werden einzelne modulierte Trägerfrequenzen zu einem breitbandige Signal zusammengefasst, wird eine solche Vorgehensweise als Frequenzmultiplexing bezeichnet. 


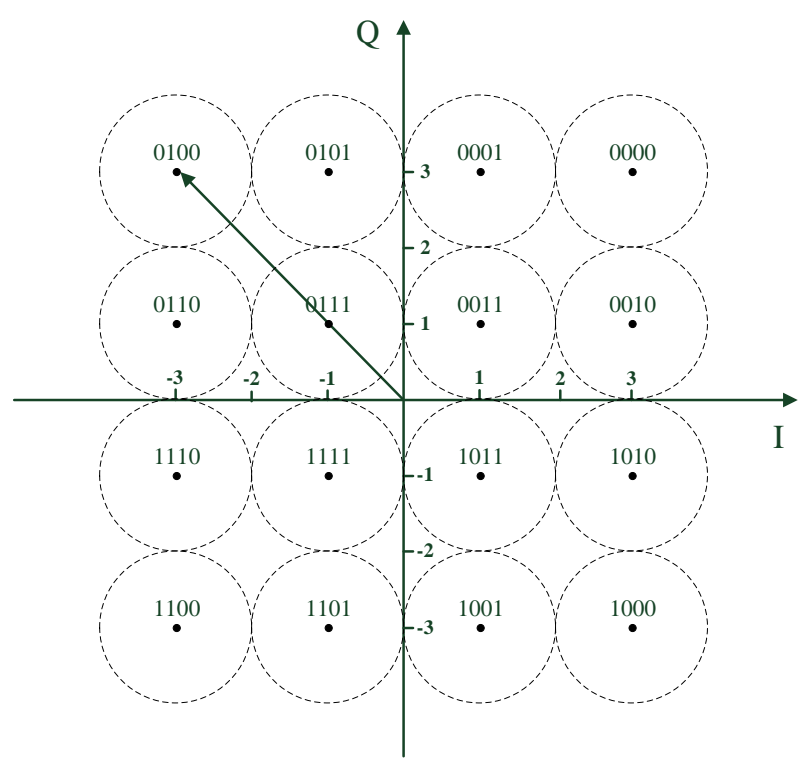

Abbildung 4.4.: Signalraumkonstellation 16-QAM [230].

\subsubsection{Orthogonales Frequenzmultiplexverfahren}

Bei dem in der vorliegenden Arbeit zur Anwendung kommenden orthogonalen Frequenzmultiplexverfahren OFDM handelt es sich um eine Sonderform von FDM (engl.: Frequency-Division Multiplexing), bei der die Trägerfrequenzen der Unterkanäle zueinander eine orthogonale Abhängigkeit bilden. Orthogonalität bedeutet in dem Zusammenhang, dass alle Frequenzen der Unterkanäle $f_{n}$ ganzzahlige Vielfache einer Basis- oder Grundfrequenz $f_{g}$ sind. Die verfügbare Kanalbandbreite $f_{B}$ wird dabei auf $N$ Unterkanäle mit jeweils gleicher Bandbreite aufgeteilt, Gl. (4.8). Die Orthogonalität der Träger bewirkt, dass sich die Trägersignale kaum gegenseitig beeinflussen, da die Amplituden aller anderen Träger bei der Betrachtung der Mittenfrequenz der einzelnen Träger $f_{g}, 2 f_{g}, \ldots$, außer dem gerade betrachteten Träger selbst, alle Null sind.

$$
f_{g}=\frac{f_{B}}{N}, f_{n}=n f_{g}, n=0,1, \ldots, N-1
$$

In Abbildung 4.5 ist die spektrale Anordnung der einzelnen $N$ Unterträger und das resultierende Summenspektrum dargestellt.

Das eigentliche Prinzip bzw. die Möglichkeiten des OFDM-Verfahrens bestehen darin, die hohe serielle Datenrate des ursprünglich zu sendenden Signals in parallele langsamere Teildatenströme aufzuteilen. Durch die Aufteilung der einzelnen Datenströme auf die sich nicht gegenseitig beeinflussenden orthogonalen Unterträger und 


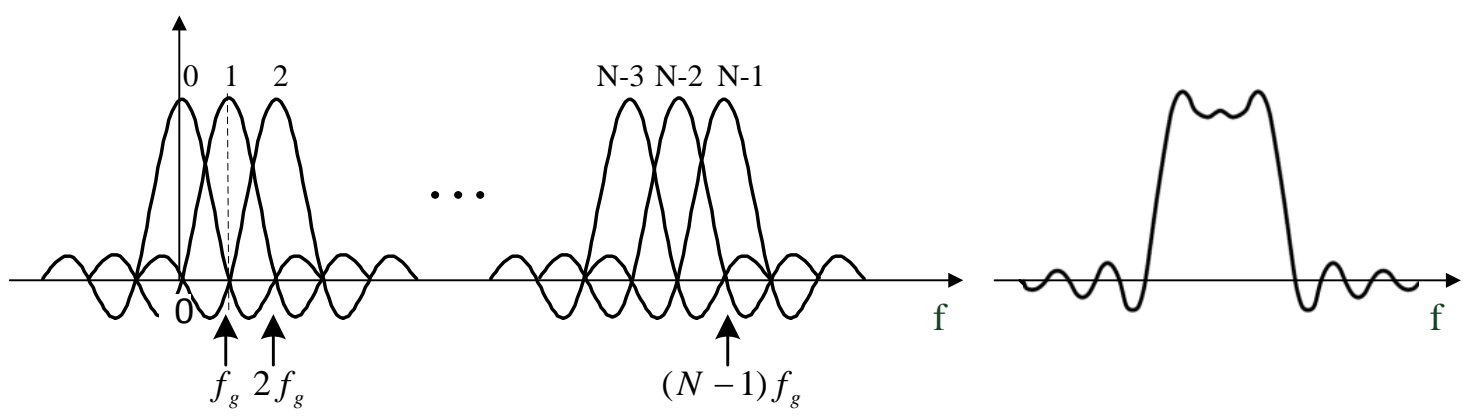

Abbildung 4.5.: Spektrum der Unterträger mit Summenspektrum bei OFDM [80].

der sich daraus resultierenden längeren Symboldauer bei der Datenübertragung hat OFDM Vorteile gegenüber anderen Multiplexverfahren.

Bei stark stör- und echobehafteten Übertragungsstrecken ist das OFDM-Verfahren besonders robust gegen Impulsstörungen. Ein Nachteil von OFDM ist die benötigte hohe Rechenleistung bei der Signalerzeugung durch den Einsatz der IFFT (engl.: Inverse Fast Fourier-Transformation) im Sendebereich und der FFT (engl.: Fast Fourier-Transformation) im Empfangsbereich [141]. Allerdings ist es durch die Verfügbarkeit immer leistungsfähigerer und kostengünstigerer Hardware möglich, z.B. durch Verwendung von digitalen Signal-Prozessoren (DSPs), ASICs (engl.: Application-Specific Integrated Circuit) oder FPGA-basierten Hardwarestrukturen, die sehr rechenintensive Bearbeitung in Echtzeit für die Modulation und Demodulation durchzuführen.

Ein weiterer Vorteil des OFDM-Verfahrens besteht darin, dass durch die schmalbandige Mehrträgerübertragung ein frequenzselektiver Übertragungskanal so in schmalbandige Unterkanäle aufgeteilt werden kann, dass sich näherungsweise nichtselektive Unterkanäle bilden. Treten auf dem Übertragungskanal typische schmalbandige Störungen auf, können entsprechende Trägerfrequenzen ausgeblendet werden. Die Signalübertragung des gestörten Kanals kann von einem anderen weniger gestörten freien Kanal übernommen werden. Der Sachverhalt findet Anwendung für die Umsetzung der Datenübertragung und Datensynchroniserung in Abschnitt 4.3.3.

Das Prinzip des OFDM-Verfahrens ist in der Nachrichtentechnik schon länger bekannt. OFDM basierte Verfahren werden z.B. im neuen digitalen terrestrischen Rundfunkstandard DAB (engl.: Digital Audio Broadcasting), in der digitalen terrestrischen Fernsehtechnik DVB (engl.: Digital Video Broadcasting) oder im Bereich der WLANFunknetze eingesetzt [94]. 


\subsubsection{Aufbau des Datenübertragungssystems}

In Abbildung 4.6 ist die grundsätzliche Funktionsweise eines OFDM-Übertragungssystems beschrieben, wie es im PLC-Modem laut Abschnitt 4.3.1 Verwendung findet. Zum besseren Verständnis der in Abschnitt 4.4 implementierten Softwarestrukturen wird nachfolgend auf den Aufbau des Datenübertragungssystems eingegangen.

Auf der Sendeseite des OFDM-Übertragungssystems (in Abbildung 4.6 links oben) wird die Sequenz der zu übertragenden seriellen Bitfolge $a(n)$ zuerst durch den Multiplexer in $N$ parallel Datenströme $b(n)$ zerlegt. Die OFDM-Symboldauer $T_{S y m}$ verlängert sich dadurch gegenüber einem Einträgersystem mit vergleichbarer Gesamtdatenrate, Gl. (4.9).

$$
T_{S y m}=N T_{E T}, T_{E T}=\frac{1}{f_{E T}} .
$$

$T_{E T}$ steht in Gl. (4.9) für das Symbolintervall eines Einzelträgersystems, die Symbolrate des OFDM-Systems wird dabei um den Faktor N reduziert. Durch die Wahl von $N$ kann das Symbolintervall $T_{\text {Sym }}$ auch so gewählt werden, dass, bezugnehmend auf die Dauer der Kanalimpulsantwort, der Einfluss der Intersymbolinterferenz auch ohne Schutzintervall minimiert werden kann (siehe Abschnitt 4.3.3).

Nach der Aufteilung des Datenstromes durch den Multiplexer folgt für jeden Kanal das Symbol-Mapping, d.h. die Zuordnung zu einem komplexen $M$-wertigen Datensymbol $\mathbf{x}(n)[95]$.

$$
b(n) \in\{0,1\} \rightarrow \mathbf{x}(n) \in\left\{\mathbf{x}_{0}, \mathbf{x}_{1} \ldots \mathbf{x}_{M-1}\right\}, \quad \text { mit } \log _{2} M=q_{n} .
$$

$q_{n}$ beschreibt dabei die Anzahl der Bit für den $n$-ten Datenstrom. Nach dem Mapping werden die Datensymbole $\mathbf{x}(n)$ der IDFT (Inverse Diskrete Fourier-Transformation) unterzogen und auf $N$ orthogonale Unterkanäle moduliert. Die IDFT stellt eine Fourier-Synthese dar, d.h. jedem Bit oder jeder Bitkombination am Eingang des IDFT-Blocks kann eine diskrete Frequenz mit diskreter Amplitude- und Phasenlage zugeordnet werden, es erfolgt somit eine Transformation vom Frequenz- in den Zeitbereich.

Es ist theoretisch auch möglich, einzelne schmalbandige Signalgeneratoren der Trägerfrequenzen $f_{T}(0) \ldots f_{T}(N-1)$ durch die komplexen Datensymbole $\mathbf{x}(n)$ anzusteuern bzw. zu modulieren. Das Ausgangssignal setzt sich dann aus der Summe der Einzelsignale zusammen. Allerdings ist schon alleine der Aufwand für die Realisierung der Hardware sehr groß. Der IDFT-Block ersetzt somit die riesige Anzahl von Multiplizierer und Oszillatoren sehr elegant. Zusätzlich ergibt sich durch die Orthogonalität der Trägerfrequenzen ein vereinfachtes Verfahren. Gl. (4.11) beschreibt den 


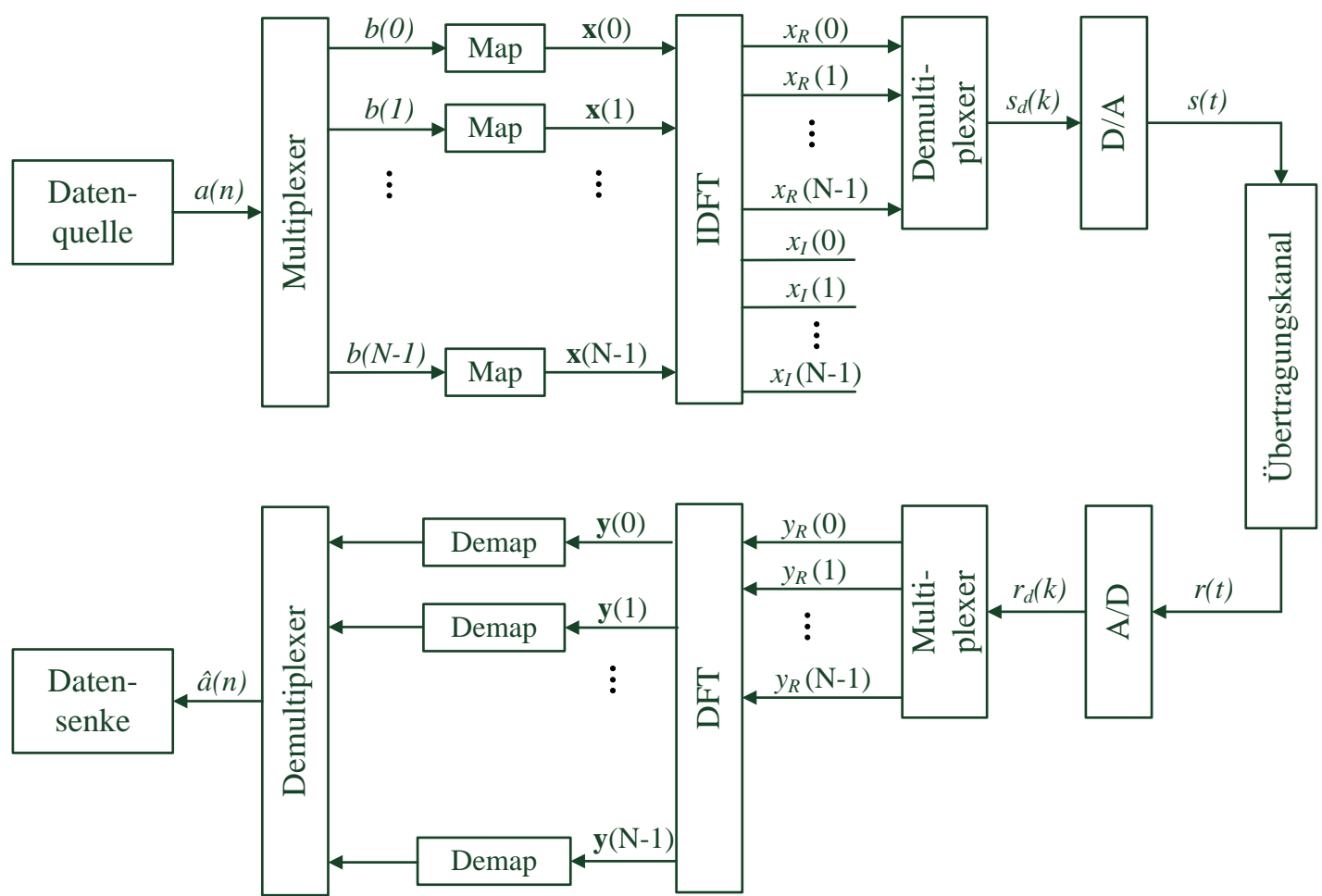

Abbildung 4.6.: Blockschaltbild des gesamten OFDM-Datenübertragungssystems [96].

Algorithmus der IDFT bzw. die Generierung des OFDM-Signales für den zu übertragenden digitalen Datenstrom. Der Wert $T_{S}$ in Gl. (4.11) steht für das Zeitintervall der diskreten Abtastung [80].

$$
\mathbf{x}\left(k T_{S}\right)=\frac{1}{N} \sum_{n=0}^{N-1} \mathbf{x}\left(\frac{n}{N T_{S}}\right) e^{j \frac{2 \pi n}{N} k}, \quad k=0,1, \ldots, N-1
$$

Die IDFT wie auch die DFT (Diskrete Fourier-Transformation) erlauben eine variable Blocklänge am Ausgang des Multiplexers, der Rechenaufwand ist allerdings sehr groß. So erfordert die Auswertung $N^{2}$ komplexe Multiplikationen und $N(N-1)$ komplexe Additionen.

Für die praktische Umsetzung der Fourier-Synthese bzw. DFT finden daher die bereits erwähnte FFT und die IFFT Verwendung. Die FFT ist eine auf Geschwindigkeit optimierte DFT wie auch entsprechend die IFFT. Allerdings benötigen FFT und IFFT immer eine Blocklänge, die sich als 2er Potenz ergibt, was praktisch aber keinen Einfluss hat, da nicht belegte Eingänge notfalls mit dem Wert Null belegt werden können. Zur Reduzierung des Rechenaufwandes sind verschiedene FFT-Algorithmen 
für die Implementierung der DFT bekannt [30]. Eine zeiteffiziente Berechnung ermöglicht der Cooley-Tukey-Algorithmus [41].

Ist die eine Hälfte der Unterkanäle nicht mit Daten moduliert, ist der Ausgangsdatenstrom $\mathbf{x}\left(k T_{S}\right)$ der IDFT analytisch. Das Spektrum von $\mathbf{x}\left(k T_{S}\right)$ für negative Frequenzen verschwindet, Gl. (4.12) [80], [221].

$$
\mathbf{x}\left(\frac{n}{N T_{S}}\right)=0, \text { wenn } n \geq \frac{N}{2}
$$

Der Imaginärteil eines analytischen Signals ist die Hilbert-Transformation des Realteils, d.h. der Imaginärteil von $\mathbf{x}\left(k T_{S}\right)$ wird für die Übertragung selbst nicht benötigt, Gl. (4.13) [95].

$$
\Im\left[\mathbf{x}\left(k T_{S}\right)\right]=\mathcal{H}\left\{\Re\left[\mathbf{x}\left(k T_{S}\right)\right]\right\}
$$

Der Demultiplexer im Sendezweig in Abbildung 4.6 ordnet daher nur den Realteil von $\mathbf{x}\left(k T_{S}\right)$ dem diskreten OFDM-Symbol $s_{d}\left(k T_{S}\right)$ zu. Daraus folgt Gl. (4.14) [80]. In der Gleichung ist $\mathbf{x}\left(n / N T_{S}\right)^{*}$ die konjugiert-komplexe Größe zu $\mathbf{x}\left(n / N T_{S}\right)$.

$$
\begin{aligned}
s_{d}\left(k T_{S}\right) & =\Re\left[\mathbf{x}\left(k T_{S}\right)\right] \\
& =\frac{1}{N} \sum_{n=0}^{N-1} \Re\left\{\mathbf{x}\left(\frac{n}{N T_{S}}\right) e^{j \frac{2 \pi n}{N} k}\right\} \\
& =\frac{1}{2 N} \sum_{n=0}^{N-1}\left\{\mathbf{x}\left(\frac{n}{N T_{S}}\right) e^{j \frac{2 \pi n}{N} k}+\mathbf{x}\left(\frac{n}{N T_{S}}\right)^{*} e^{j \frac{2 \pi(N-n)}{N} k}\right\}
\end{aligned}
$$

für $\quad k=0,1, \ldots, N-1$.

Im D/A-Wandler wird das diskrete Signal $s_{d}\left(k T_{S}\right)$ schließlich in das zeit- und wertkontinuierliche Sendesignal überführt. Auf der Empfangsseite erfolgt die Abtastung und Quantisierung des analogen Empfangssignals $r(t)$ im A/D-Wandler. Der Multiplexer im Empfänger wandelt den seriellen Datenstrom dann wieder zurück in einen parallelen Datenstrom. Durch Verwendung der DFT, Gl. (4.15) bzw. durch Verwendung der schnelleren FFT kann das Datensignal wieder zurückgewonnen werden [80].

$$
\mathbf{x}\left(\frac{n}{N T_{S}}\right)=\sum_{k=0}^{N-1} x\left(k T_{S}\right) e^{-j \frac{2 \pi k}{N} n}, \quad n=0,1, \ldots, N-1
$$




\subsection{Realisierung der Datenkommunikation}

Die bidirektionale Datenkommunikation zwischen der stationären und beweglichen Seite der induktiven kontaktlosen Energieübertragung im Versuchsaufbau (siehe Kapitel 3) wird hardwareseitig durch die Verwendung des nachfolgend beschriebenen PLC-Modems realisiert. Die Modem auf der stationären Seite und der beweglichen Seite sind baugleich und unterscheiden sich nur durch ihre programmseitige Parametrierung [80]. Jedes Modem ist mit einem separaten Sende- und Empfangskopf bzw. einem separaten Sende- und Empfangszweig zur Datenübertragung ausgestattet.

Sende- und Empfangsübertragerkopf sind in U-förmiger Bauform ausgeführt. Die Übertragerköpfe sind auf der beweglichen Seite mechanisch präzise über einen der Linienleiter (Hin- oder Rückleiter) geführt. Zur besseren magnetischen Kopplung ist der Kopf aus Ferritplättchen aufgebaut. Durch ein aufgewickeltes Spulenpaar wird die induktive Kopplung zum Linienleiter hergestellt. Die Abbildung 5.32 in Kapitel 5 zeigt einen ersten Aufbau für den Test der Datenübertragung über den Linienleiter. Hier sind die Komponenten (Modemplatine 1 [4], Modemplatine 2 [5], Sendeübertragerkopf 1 [6], Empfangsübertragerkopf 1 [7], Sendeübertragerkopf 2 [8], Empfangsübertragerkopf 2 [9]) für den Testaufbau in den Versuchsaufbau der Motorsteuerung integriert. Abbildung 4.7 zeigt den prinzipiellen Aufbau des Systems mit kontaktloser induktiver Energieübertragung und integrierter Datenkommunikation.

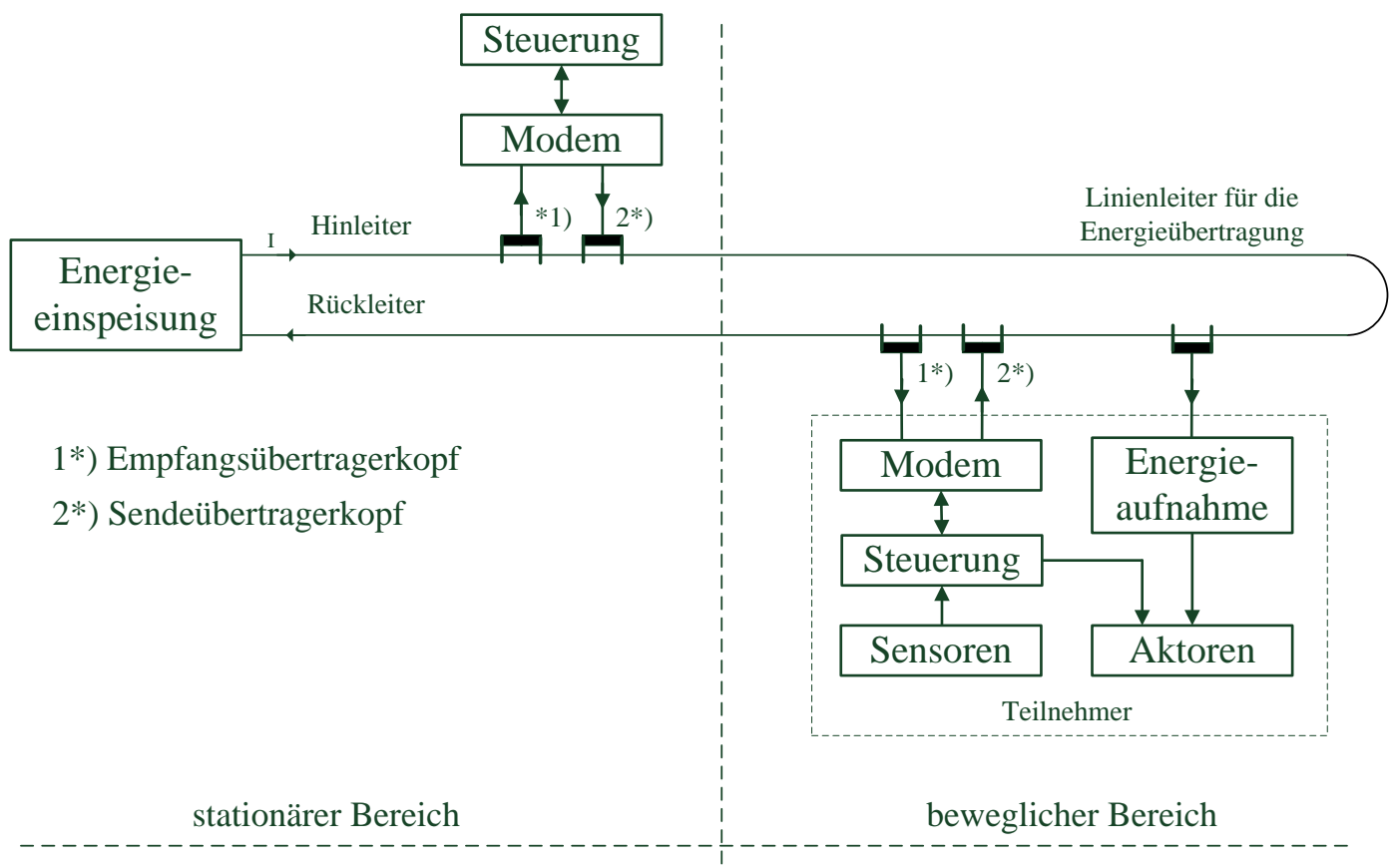

Abbildung 4.7.: Datenkommunikation mit PLC-Modem. 


\subsubsection{Aufbau des Modems}

Die Hauptkomponenten des PLC-Modems sind, wie in Abbildung 4.8 prinzipiell dargestellt, Mikrocontroller $(\mu \mathrm{C})$, FPGA und das analoge Frontend (AFE). Über die RS485-Schnittstelle kann mittels PC, Umrichter oder einem sonstigen Kommunikationsteilnehmer auf die Register- und Datenspeicher des Mikrocontrollers zugegriffen werden.

Die zentrale Aufgabe des Mikrocontrollers ist dabei die Anpassung und Synchronisation der Protokolle zwischen dem Kommunikationsteilnehmer und dem FPGA. Weiterhin werden die Sollwerte für die Sendeamplituden und die Phasenlagen eines jeden Unterträgers für die OFDM-Modulation im Mikrocontroller hinterlegt (vgl. Abschnitt 4.3.3). Somit ist es auch möglich, die Kanaldämpfung optimal auszugleichen. Die Anbindung des Mikrocontrollers an den FPGA wird über eine schnelle HS-SSCSchnittstelle (engl.: High Speed-Synchronous Serial Channel) mit bis zu $20 \mathrm{Mbit} / \mathrm{s}$ realisiert. Dabei fungiert der Mikrocontroller als Master, der FPGA als Slave. Die Synchronisation der Paketübermittlung wird über ein Handshake-Verfahren sichergestellt [54], [183].

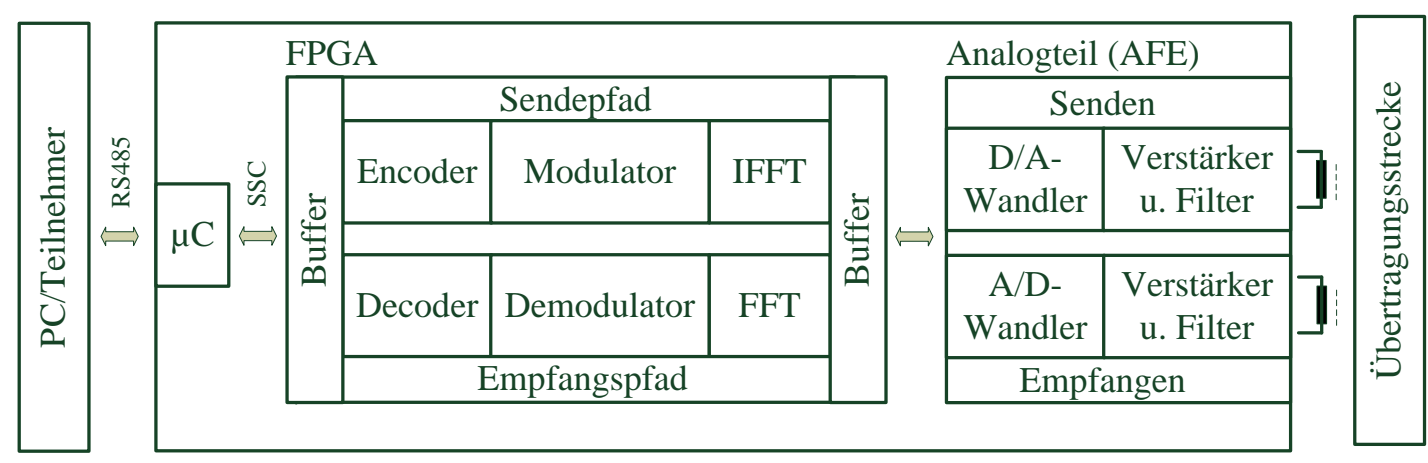

Abbildung 4.8.: Prinzipieller Aufbau des PLC-Modems.

Der verwendete FPGA im Modem vom Typ EPC3C16 - Cyclone III verfügt über 15.408 Logikzellen bzw. Logikelemente (LEs), 346 I/0-Anschlüsse mit 56 Multiplizierer und 56 M9K-Memory Blöcken. Über vier PLLs kann die Taktfrequenz des FPGAs um ein Vielfaches an die Referenzfrequenz des Oszillators angepasst werden [5]. Der FPGA wird dabei in der taktsynchronen Betriebsart (Time-Triggered) betrieben. Gegenüber der taktasynchronen Betriebsweise (Event-Triggered) hat die gewählte Betriebsart zwar eine geringere Recheneffizienz, dafür können aber alle Systemzustände deterministisch festgelegt werden.

Innerhalb des FPGAs sind, wie bereits angesprochen, Sende- und Empfangspfad separat aufgebaut. Die zu übertragenden Daten werden zuerst im Encoderblock mittels eines Viterbi-Algorithmus [92], [95] kodiert und dann zwischengespeichert. Der 
anschließende Modulator arbeitet nach dem DQPSK-Verfahren (engl.: Differential Quaternary Phase Shift Keying). DQPSK ist eine Quadratur-Phasenumtastung, die mit den Phasenwechsel der Trägerfrequenz arbeitet. Dabei können 2 Bit pro Kanal eindeutig zugeordnet werden [70]. Nach der Modulation werden die zu übertragenden Daten einer Inversen Fast Fourier-Transformation unterzogen, was einer Transformation vom Frequenzbereich in den Zeitbereich entspricht - anschließend im Sendespeicher zwischengepuffert. Der Analogteil mit D/A-Wandler, Filter und integriertem Sendeverstärker bildet letztendlich die Schnittstelle im Sendepfad zwischen FPGA und Übertragungskanal. Ein- sowie auch Ausgangsverstärker sind dabei als differenzielle Verstärker aufgebaut, wodurch es möglich ist, Gleichtaktstörungen zu unterdrücken.

Das generierte zeit- und wertkontinuierliche Signal wird dann über den Ausgangsfilter über den Übertragerkopf induktiv auf den Linienleiter eingekoppelt. Die primäre Aufgabe des Filters ist dabei, den induktiven Einfluss des Energiestromes, maximal bis zu $85 \mathrm{~A}$ bei einer Nennfrequenz von $25 \mathrm{kHz}$ mit seinen Oberschwingungen, zu entkoppeln. Der Energiestrom und seine Oberschwingungen sind dabei zwei der größten Störeinflüsse auf dem Datenübertragungskanal selbst.

Im Empfangspfad wird das OFDM-Signal zunächst vom Empfangsübertragerkopf ausgekoppelt und einem analogen passiven Eingangsfilter zugeführt. Das Eingangsfilter hat dabei Hochpasscharakter, um die Einflüsse des $25 \mathrm{kHz}$-Energiestromes und seiner Oberschwingungen auf das OFDM-Signal, das in einem Frequenzband zwischen ca. $500 \mathrm{kHz}$ und $2.5 \mathrm{MHz}$ arbeitet, auszuschließen bzw. zu begrenzen. Das hochpassgefilterte Signal wird dann entsprechend Abbildung 4.8 an den EmpfangsEingangsverstärker zugeführt und vom A/D-Wandler in einen digitalen Datenstrom konvertiert.

Im Empfangspfad des FPGAs findet, entsprechend dem beschriebenen Ablauf im Sendepfad, die Invertierung der Signalverarbeitung statt. Zunächst werden die Daten im Empfangspuffer gespeichert, dann erfolgt die Transformation der Datensignale vom Zeitbereich in den Frequenzbereich mittels FFT mit anschließender Zwischenspeicherung der berechneten Werte für die Demodulation. Nach der Demodulation der Empfangsdaten mit dem DQPSK-Modulationsverfahren und Dekodierung im Encoderblock unter Verwendung der Viterbi-Decoderierung [92], [95] werden die Daten an den Mikrocontroller übergeben und über die SSC-Schnittstelle dem Teilnehmer zur Verfügung gestellt. Abbildung 4.9 zeigt das Layout des verwendeten Modems.

\section{SSC-Schnittstelle}

Über die SSC-Schnittstelle wird die bidirektionale Kommunikation zwischen Mikrocontroller und FPGA realisiert. Der prinzipielle Ablauf ist in Abbildung 4.10 dar- 


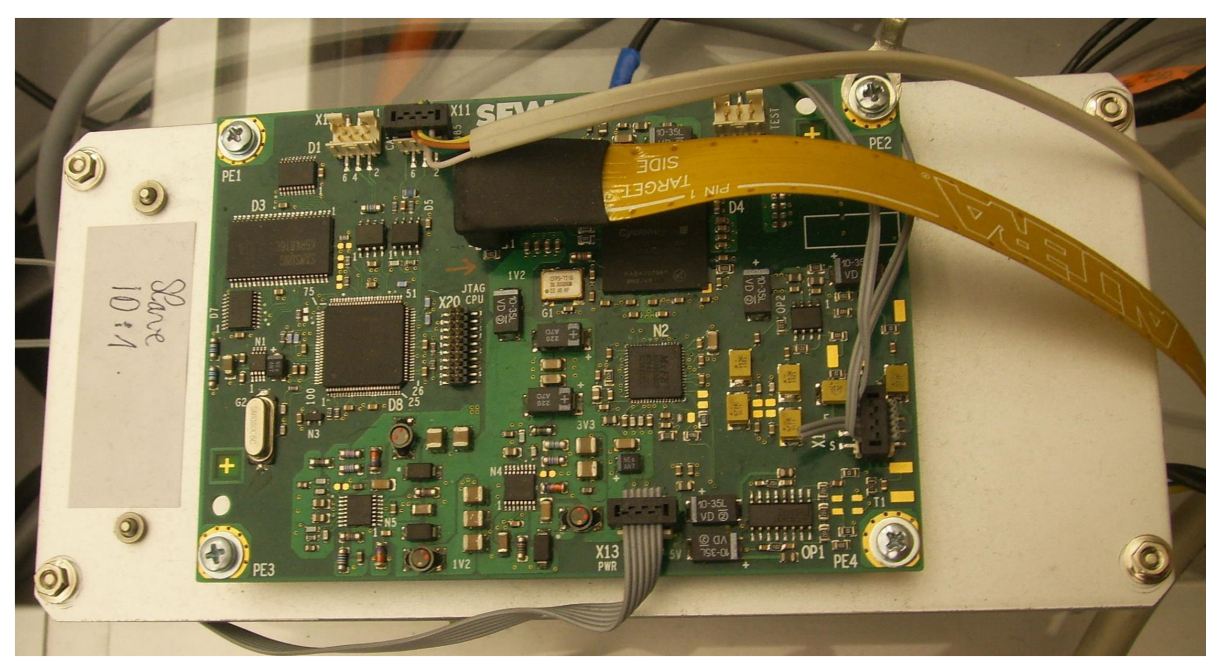

Abbildung 4.9.: Layout des PLC-Modems [80].

gestellt. Der SSC-Takt wird durch den Mikrocontroller generiert. In der SSC-TaktPhase werden die Daten vom FPGA übernommen. Nach der Übergabe der Daten an den FPGA sendet der FPGA ein Request-Signal (Wartesignal) an den Mikrocontroller - während der Wartezeit werden die Daten dann vom FPGA weiterverarbeitet.

Umgekehrt ist es möglich, durch eine Interrupt-Anforderung an den Mikrocontroller Daten vom FPGA an den Mikrocontroller zu senden. Der Aufbau der SSCDatenpakete mit den einzelnen Registern ist in Tabelle 4.1 beschrieben. Ein 16-Bit breites Datenwort beinhaltet die Nutzdaten (Datenregister), ein zweites 16-Bit breites Steuerwort die Konfigurationsdaten bzw. die Konfigurationsbit.
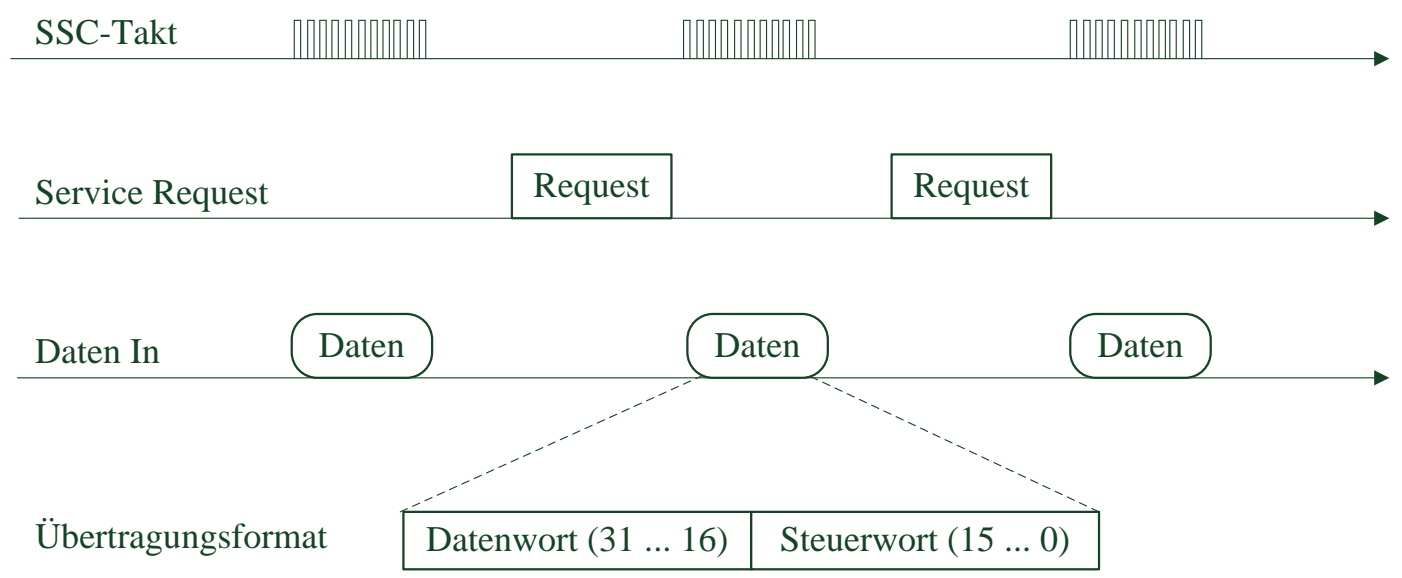

Abbildung 4.10.: SSC-Schnittstelle [80]. 
Tabelle 4.1.: Daten- und Konfigurationsregister [80]

\begin{tabular}{|c|c|c|c|}
\hline Bezeichnung & Typ/Art & Bit & Beschreibung \\
\hline $\mathrm{ADDR} / \mathrm{CDATA}$ & $\begin{array}{l}\text { Adress-/Konfi- } \\
\text { gurationsdaten }\end{array}$ & $0 \ldots 11$ & $\begin{array}{l}\text { Identifiziert die Adresse, falls } \\
\text { das AF-Flag gesetzt ist, an- } \\
\text { sonsten Konfigurationsdaten }\end{array}$ \\
\hline $\mathrm{R} / \mathrm{W}$ & $\begin{array}{l}\text { Schreib-/Lese- } \\
\text { Modus }\end{array}$ & 12 & $\begin{array}{l}\text { Identifiziert einen Lese- oder } \\
\text { Schreibvorgang }\end{array}$ \\
\hline $\mathrm{AF}$ & Adress-Modus & 13 & $\begin{array}{l}\text { Identifiziert eine Adresse, falls } \\
\text { das AF-Flag aktiv ist, ansons- } \\
\text { ten Konfigurationsdaten }\end{array}$ \\
\hline $\mathrm{CF}$ & $\begin{array}{l}\text { Konfigurations- } \\
\text { Modus }\end{array}$ & 14 & $\begin{array}{l}\text { Identifiziert gültige Konfigura- } \\
\text { tionsdaten }\end{array}$ \\
\hline $\mathrm{DF}$ & Daten-Modus & 15 & Identifiziert gültige Daten \\
\hline TX Data & Datenregister & $16 \ldots 31$ & $\begin{array}{l}\text { Nutzdaten für die Übertra- } \\
\text { gung }\end{array}$ \\
\hline
\end{tabular}

\subsubsection{Master-Slave-Betrieb}

Der Kommunikationsablauf zwischen den Teilnehmern ist nach dem Master-SlavePrinzip aufgebaut. Das Master-Modem ist, wie in Abbildung 4.7 dargestellt, stationär, also feststehend montiert. Das Slave-Modem ist auf der beweglichen Seite angeordnet. In der Abbildung 4.7 ist exemplarisch nur ein beweglicher Teilnehmer eingezeichnet. Die Anzahl der beweglichen Teilnehmer kann modular, bei gleichem Aufbau, entsprechend erhöht werden.

Beim Master-Slave-Betrieb verwaltet das Master-Modem die Zugriffsrechte auf den Datenkanal - hier zu allen Teilnehmern auf dem Linienleiter. Eine direkte Kommunikation zwischen den einzelnen Slaves ist dabei nicht im Protokoll vorgesehen. Um ein deterministisches Verhalten der Kommunikation sicherzustellen bzw. eine konstante Datenrate zu jedem Teilnehmer zu gewährleisten, findet das synchrone Zeitmultiplex-Verfahren (STD, engl.: Synchronous Time Division) Verwendung. Beim STD-Verfahren weist der Master einen festen Zeitabschnitt jedem Slave für die Übertragung der Daten zu. Der Slave antwortet unmittelbar nach dem Erhalt der Nachricht innerhalb des definierten Zeitfensters. 


\section{Control-Register}

Beim Datenaustausch über den Linienleiter wird dem Datentelegramm im Steuerkanal ein 16 Bit breites Control-Register mit übertragen. Über die ersten sieben Bit des Registers wird die Telegrammlänge bzw. die Anzahl der zu übertragenden OFDMSymbole übermittelt. Bit 8-13 steuert die Quelle-Ziel-Beziehung zwischen Masterund Slave-Modem, wenn das Master-Bit 14 den Zustand Eins erfüllt. Nach dem Einlesen der Empfangsdaten kann jeder Slave-Teilnehmer durch Vergleich seiner eigenen zugeordneten hinterlegten Slave-ID-Nummer feststellen, ob die Daten für ihn bestimmt sind. Ist das Master-Bit nicht gesetzt, wird in Bit 8-13 der ChannelIndex übergeben. Er gibt an, welche Trägerfrequenzen auf dem Linienleiter für die Übermittlung der Daten vom Slave an den Master verwendet werden. Das ControlRegister ist in Tabelle 4.2 dargestellt.

Tabelle 4.2.: Control-Register im Steuerkanal [80].

\begin{tabular}{ll}
\hline Bit & Beschreibung \\
\hline $0 \ldots 6$ & Telegrammlänge \\
\hline 7 & Reserve \\
\hline $8 \ldots 13$ & Channel-Index/Slave-ID \\
& Master-Bit $=0:$ aktuell gewählte Trägerfrequenzen \\
& Master-Bit $=1:$ aktueller Slave-Teilnehmer \\
\hline 14 & Master-Bit \\
& Bit $=0:$ Daten von Slave an Master \\
& Bit $=1:$ Daten von Master an Slave \\
\hline 15 & Reserve \\
\hline
\end{tabular}

\subsubsection{Parameter des Datenübertragungssystems}

Bei der Datenübertragung kann die Orthogonalität der Träger eines OFDM-Signals durch Intersymbol-Interferenzen (ISI) gestört werden [80]. Intersymbol-Interferenzen sind Störungen durch Symbolübersprechen bei der Übertragung zeitlich aufeinanderfolgender Sendesymbole.

Wird ein Schutzintervall, wie in Abbildung 4.11 dargestellt, eingefügt, können Verzerrungen des Datensignals vermieden werden. Im Schutzintervall selbst werden keine Daten übertragen. Daraus resultiert eine Reduzierung der maximalen möglichen Datenrate aber auch eine Reduzierung der Fehlerrate. Das Schutzintervall wird bestimmt durch die Dauer der Impulsantwort der Übertragungsstrecke, d.h. die Dauer 
des Schutzintervalls muss mindestens der Dauer der Impulsantwort entsprechen.

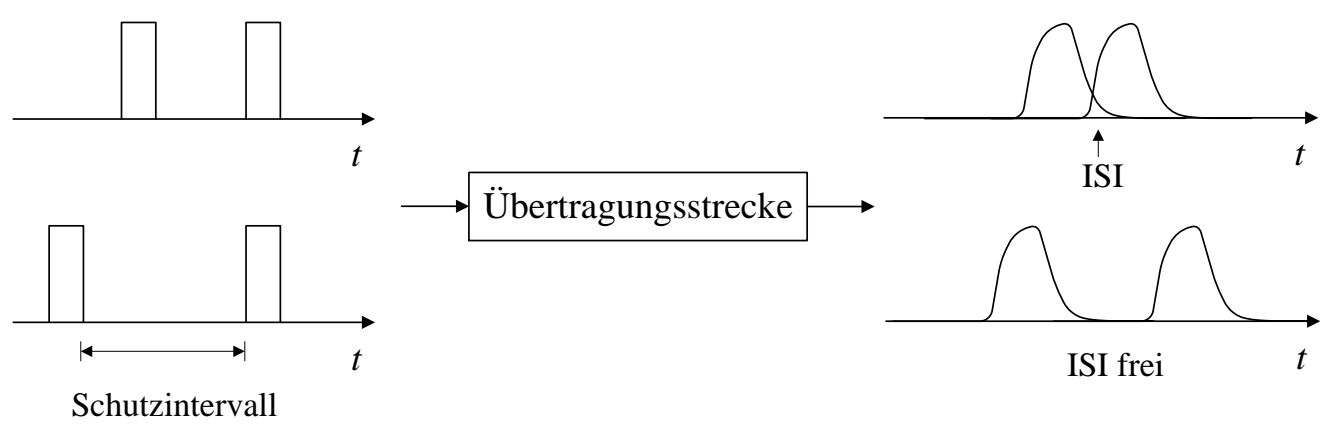

Abbildung 4.11.: Schutzintervall gegen Intersymbol-Interferenz [80].

Die Impulsantwort stellt die Reaktion der Übertragungsstrecke auf einen DiracImpuls als Eingangssignal dar. In der Praxis lässt sich der ideale Dirac-Impuls allerdings nur annähern. Zur Bestimmung der Kanalimpulsantwort wurde in einer Simulation ein Rechteckimpuls der Dauer $T_{\text {Imp }}=30 \mathrm{~ns}$ mit der Amplitude $U_{A m p}=1 \mathrm{~V}$ am Eingang des Übertragungskanals angelegt. Die Übertragungseigenschaften des Kanals entsprechen dabei des in Abschnitt 3.4 beschriebenen Versuchsaufbaus. Die Vorgehensweise für die Bestimmung der Kanalimpulsantwort entspricht dem in [80] vorgestellten Verfahren. In der Simulation konnte für die Kanalimpulsantwort ein Wert von $T_{\text {ImpK }}<4 \mu s$ ermittelt werden.

Bei der praktischen Realisierung des Schutzintervalls wird ein Teil des vorderen OFDM-Symbols kopiert und am Ende des OFDM-Symbols angefügt. Die gesamte Symboldauer $T_{S y m}$ in Gl. (4.16) resultiert dann aus der Summe der effektiven Symboldauer $t_{S y m(e f f)}$ und der Dauer des Schutzintervalls $t_{D S}$.

$$
T_{\text {Sym }}=t_{\text {Sym }(e f f)}+t_{D S} .
$$

Die effektive OFDM-Symboldauer $t_{\text {Sym(eff) }}$ ist mit 15,4 $\mu \mathrm{s}$ gewählt (vgl. Abbildung 4.12). Entsprechend den Orthogonalitätsbedingungen für die Trägerfrequenzen resultiert daraus die Grundschwingung bzw. der Unterträgerabstand von $\Delta f=65 \mathrm{kHz}$. Das Schutzintervall $t_{D S}$ wurde auf einen Wert von 4,6 $\mu$ s festgelegt.

Die gesamte Dauer eines OFDM-Symbols mit Schutzintervall $T_{\text {Sym }}$ beträgt dann $20 \mu \mathrm{s}$, was vorteilhaft ist, da zur Synchronisation die Nulldurchgänge des $25 \mathrm{kHz}$ Energiestromes verwendet werden können. Insgesamt ergeben sich für das OFDMÜbertragungssystem 32 Trägerfrequenzen. Kanal 1: $65 \mathrm{kHz}$, Kanal 2: $130 \mathrm{kHz}, \ldots$ bis Kanal 32: $2080 \mathrm{kHz}$. Für die Datenübertragung selbst werden 23 Unterträger im 


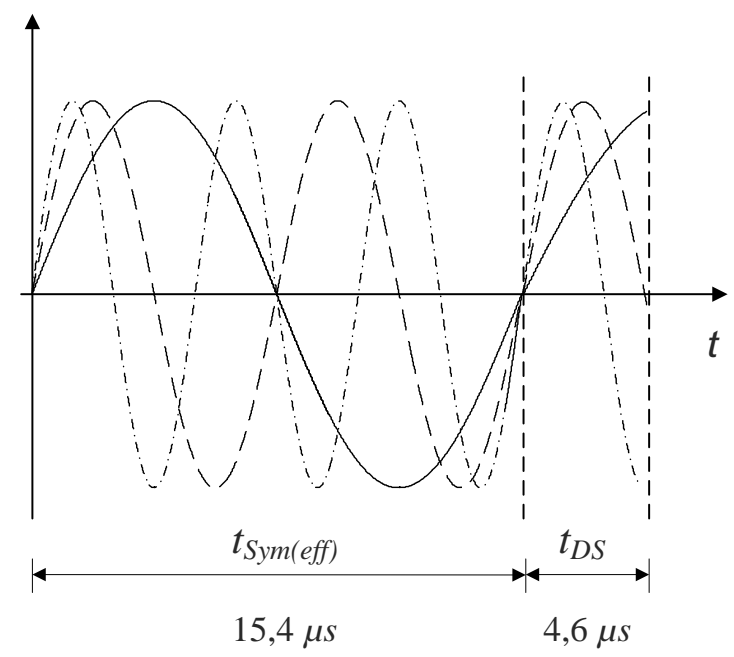

Abbildung 4.12.: OFDM-Symboldauer [80].

Frequenzbereich von $650 \mathrm{kHz} \ldots 2 \mathrm{MHz}$ verwendet, d.h. Kanal 10: $650 \mathrm{kHz} \ldots$ bis Kanal 32: $2080 \mathrm{kHz}$.

Die Trägerfrequenzen sind so gewählt, dass ausgehend vom Übertragungsverhalten des Kanals (Abbildung 3.12) der optimale Frequenzbereich für die Datenübertragung verwendet wird und dass gleichzeitig ausreichend genug Abstand zur $25 \mathrm{kHz}$ Grundfrequenz des Energiestromes besteht bzw. die Abstände der Trägerfrequenzen zu den ersten neun harmonischen Oberschwingungen des Energiestromes berücksichtigt sind.

\subsection{Darstellung des Verfahrens für die Synchronisierung der Teilnehmer}

Der folgende Abschnitt geht der Frage nach, wie bewegliche elektrische Verbraucher, die über die induktive kontaktlose Energieübertragung miteinander kommunizieren, zueinander synchronisiert werden. Durch die Erweiterung des OFDM-Datentelegramms durch einen Zeitstempel wird eine konkrete Zeitbasis für den Datenaustausch generiert. Anschließend werden im FPGA durch Parallelverarbeitung der Daten die Laufzeiten der Signale im Sende- und Empfangspfad des Modems bestimmt. Zuletzt wird die Synchronisationsgenauigkeit zwischen einem stationären Teilnehmer und einem beweglichen Teilnehmer für den konkreten Versuchsaufbau ermittelt. Im Anschluss daran wird auf die Frequenzstabilität von Oszillatoren und den Einfluss der Gangabweichung zwischen den unterschiedlichen Teilnehmern eingegangen. 


\subsubsection{Erweitertes Datentelegramm}

Der Aufbau des erweiterten Datentelegramms mit einem zu ergänzenden Zeitstempel ist in Abbildung 4.13 dargestellt. Zur Synchronisation der Teilnehmer und zur Kenntlichmachung, dass eine neue Nachricht auf dem Übertragungskanal übertragen werden soll, wird zu Beginn der Datenübertragung ein Pilotsignal (Chirp-Signal) über einen Zeitraum von ca. $60 \mu s$ vom jeweiligen Modem gesendet. Beim Pilotsignal handelt es sich konkret um einen positiven Chirp, dessen Frequenz sich zeitlich im Bereich von $500 \mathrm{kHz} \ldots 2 \mathrm{MHZ}$ ändert.

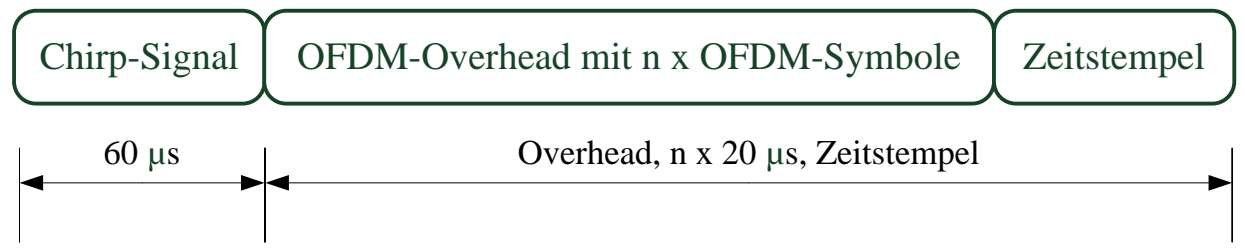

Abbildung 4.13.: Aufbau und Struktur des Datentelegramms.

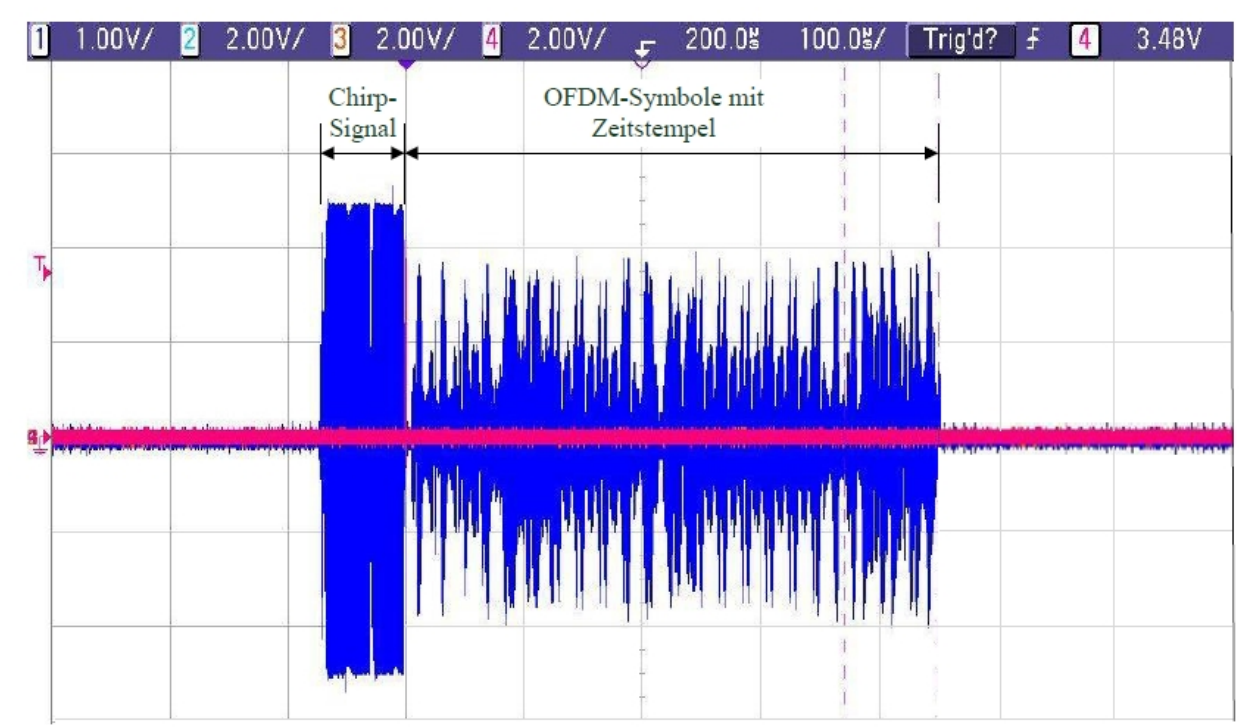

Abbildung 4.14.: Aufzeichnung des Datentelegramms.

Anschließend folgen OFDM-Overhead und die OFDM-Symbole bzw. die Nutzdaten mit eingebundenem Zeitstempel. Jedes OFDM-Symbol belegt eine Dauer von ca. $20 \mu s$. Der eingefügte Zeitstempel belegt insgesamt 4 Byte, was bei Verwendung von 4 Kanälen vier OFDM-Symbolen entspricht. Abbildung 4.14 zeigt beispielhaft das reale aufgezeichnete Signal mit dem Oszilloskop.

Die Realisierung des Zeitstempels bzw. der Uhr im FPGA ist einfach durch das 
Hintereinanderschalten mehrerer Zähler umgesetzt. Der Grundtakt wird durch den auf der Platine vorhandenen externen Oszillator mit seiner Nominalfrequenz von 20 MHZ gebildet. Dessen Takt wird durch eine PLL auf eine Systemfrequenz von 50 MHZ erhöht. Dadurch ergibt sich für den ersten und schnellsten Zähler eine Taktrate von 20 ns mit eine Bitbreite von 6 Bit (dezimale Obergrenze 50). Der erste Zähler inkrementiert im weiteren Verlauf den zweiten Zähler im $\mu s$-Bereich (10 Bit, dezimale Obergrenze 1000), der zweite Zähler entsprechend den $m s$-Zähler (10 Bit, dezimale Obergrenze 1000). Zuletzt inkrementiert der ms-Zähler den sec-Zähler (6 Bit, dezimale Obergrenze 60). Minuten- und Stundenzähler sind gleichfalls angelegt, werden im Versuchsaufbau aber nicht ausgewertet bzw. berücksichtigt, da die Wertebereiche der Zähler nicht im Zeiterfassungsbereich des betrachteten Synchronisationsintervalls liegen.

\subsubsection{Laufzeitermittlung im Sendepfad}

Der Durchlauf der Nutzdaten vom Sendeteilnehmer über die RS485-Schnittstelle zum Mikrocontroller, über die SSC-Schnittstelle zum FPGA, dann die Weiterverarbeitung der Daten im Encoder, Modulator, Unterziehen der Daten der IFFT und letztendlich die Übertragung der Daten über das analoge Frontend zum Empfangsteilnehmer ist sehr zeit- und rechenintensiv (vgl. Abbildung 4.8). Wird ein Zeitstempel oder ein Synchronisationssignal bereits schon beim Sendebeginn generiert, entstehen große Ungenauigkeiten bei der Synchronisation zwischen den Teilnehmern.

Anders als in einem klassischen Mikrocontroller, der nur sequentielle Vorgänge bearbeiten kann, ist es bei einem FPGA möglich, parallele Abarbeitungsstrukturen aufzubauen, die voneinander unabhängig arbeiten. Es ist deshalb naheliegend, die Bearbeitungszeiten im FPGA, die abhängig von der Anzahl der zu übertragenden Prozessdaten unterschiedlich sein können, durch parallel arbeitende Strukturen zu erfassen, um so die Synchronisationsgenauigkeiten zwischen den Teilnehmern zu verbessern. Abbildung 4.15 zeigt die realisierte Struktur, um die Bearbeitungszeiten innerhalb des FPGA zu erfassen bzw. rechnerisch zu kompensieren.

Im ersten Schritt werden die Prozessdaten, im konkreten Fall acht Byte, vom Mikrocontroller über die SSC-Schnittstelle übertragen. Im vorgehenden Sendezyklus $(n-1)$ wurde die Bearbeitungszeit für die Aufbereitung der Daten vom Zeitpunkt: „Daten empfangen“ bis zum Startzeitpunkt: „Datentelegramm senden“ durch einen parallel arbeitenden Laufzeitzähler bestimmt. Die ermittelte Bearbeitungszeit durch den Laufzeitzähler $t_{L}$ wird nun bei der Ausgabe des aktuellen Zeitstempels $t_{z}(n)$ berücksichtigt bzw. eingerechnet. Im allerersten Sendezyklus $(n=1)$ ist der Laufzeitwert allerdings noch nicht bekannt. Die Daten des integrierten Zeitstempels werden dann zusammen mit den regulären Nutzdaten im FPGA kodiert und weiterverarbeitet. 


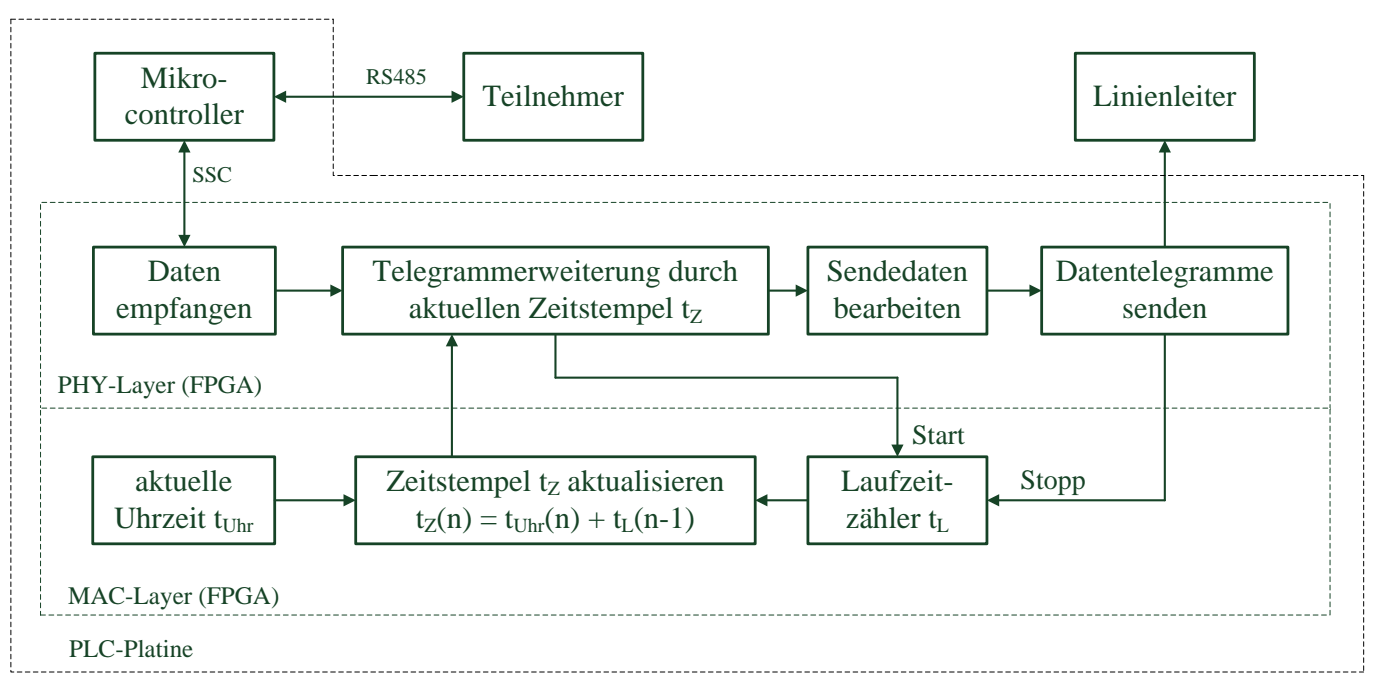

Abbildung 4.15.: Laufzeitermittlung im Sendepfad.

Zur Überprüfung der Funktion und der Genauigkeit der Laufzeitmessung wurden mehrere Laufzeiten bzw. die Bearbeitungszeiten im Sendepfad bei gleichen Ausgangsbedingungen gemessen und dokumentiert. Die Laufzeiten wurden durch parallele Messungen mit dem Debugging Programm SignalTap [140] überprüft und bestätigt. SignalTap ist ein Bestandteil der Entwicklungsumgebung des FPGA-Herstellers. Über die JTAG-Schnittstelle können in Echtzeit die internen Zustände und die zeitlichen Verläufe im FPGA beobachtet werden [5]. Für den Laufzeitwert im Sendepfad ergibt sich ein ermittelter arithmetischer Mittelwert von $70 \mu s$ innerhalb eines Toleranzfensters von $\pm 4 \mu s$.

Nachteilig wirkt sich dabei die unsynchronisierte Datenübertragung zwischen Mikrocontroller (notwendige Interrupt-Anforderung) und FPGA über die SSC-Schnittstelle aus. Optimiert werden kann die Kommunikationsanbindung durch das im Abschnitt 5.3.5 vorgestellte Konzept der Einbindung einer Soft-Core-CPU.

\subsubsection{Laufzeitermittlung im Empfangspfad}

Die Vorgehensweise für die Laufzeitermittlung im Empfangspfad ist die gleiche wie die im Abschnitt 4.4.2 beschriebene Laufzeitermittlung im Sendepfad. Auch hier werden die parallelen Abarbeitungsstrukturen des FPGAs verwendet, um die Bearbeitungszeit der Daten im Empfangspfad zu bestimmen. Abbildung 4.16 zeigt die Übersicht über die implementierten Abläufe. 


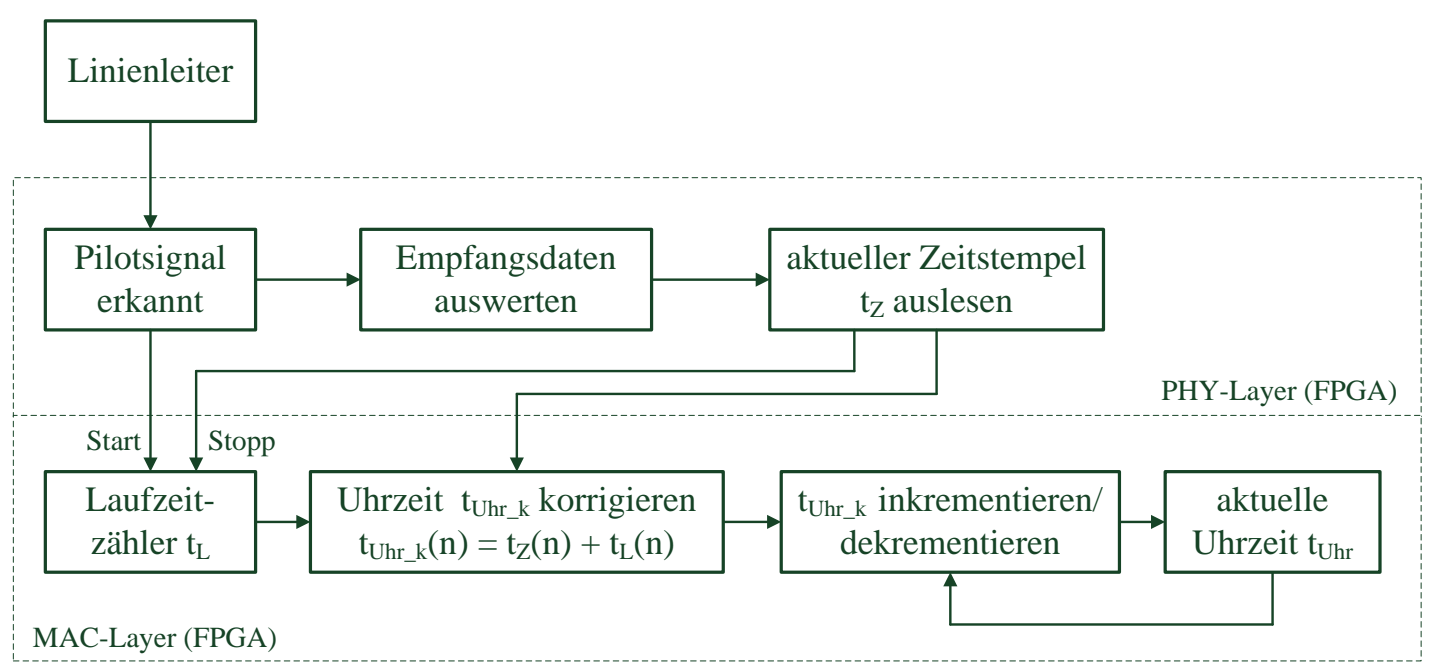

Abbildung 4.16.: Laufzeitermittlung im Empfangspfad.

Wird das Pilotsignal im Empfangspfad erkannt, wird der Laufzeitzähler $t_{L}$ gestartet. Gleichzeitig werden die Daten eingelesen, in einem Empfangspuffer zwischengespeichert und dann verarbeitet (vgl. Abschnitt 4.3.1). Ist nach der Konvertierung der Empfangsdaten der aktuelle Zeitstempel $t_{Z}$ bestimmt, wird zeitgleich der Laufzeitzähler $t_{L}$ gestoppt. In der Uhreneinheit wird anschließend der Wert für die korrigierte Uhrzeit $t_{U h r_{k}}$ aus dem aktuellen Zeitstempel $t_{Z}$ und der ermittelten Bearbeitungszeit $t_{L}$ durch den Laufzeitzähler berechnet und zwischengespeichert. Um Unstetigkeitsstellen bzw. Sprünge bei der Uhrzeitaktualisierung der Slave-Uhr zu vermeiden, wird der ermittelte Uhrzeitwert $t_{U h r_{k}}$ inkrementell durch eine Rückführung von $t_{U h r}$ an die aktuelle Uhrzeit $t_{U h r}$ angepasst.

Wie im letzten Abschnitt für die Laufzeiten im Sendepfad beschrieben, wurden auch die Laufzeiten bzw. die Bearbeitungszeiten im Empfangspfad durch den Laufzeitzähler gemessen und dokumentiert. Die Werte wurden durch parallele Messungen mit der SignalTap-Software bestätigt. Das arithmetische Mittel des ermittelten Laufzeitwertes im Empfangspfad, bei Übertragung von acht Byte Prozessdaten, wurde mit $123 \mu \mathrm{s}$ bestimmt. Für den Wert variiert der Laufzeitwert in einem Toleranzbereich von $\pm 5 \mu s$.

Negativ wirkt sich hier die nicht exakte Erfassung des Chirpsignales aus und die nicht synchronisierte Blockbearbeitung des FFT/IFFT-Blocks bei Erkennung des Chirp-Eingangssignales. 


\subsubsection{Ermittlung der Synchronisationsgenauigkeit}

Um die Synchronisationsgenauigkeit zwischen Masteruhr im Master-Modem und der Uhr in einem Slave-Modem zu bestimmen bzw. abzuschätzen, wurde ein laufzeitkorrigierter Zeitstempel im Master-Modem an den Slave verschickt. Im Slave wurde der Zeitstempel unter Berücksichtigung der Laufzeiten im Sende- und Empfangspfad an den Master zur Berechnung der Synchronisationsgenauigkeit der Uhren zurückgeschickt. Im Master-Modem wird aus dem Wert der aktuellen Masteruhr und des eingelesenen laufzeitkorrigierten Zeitstempels der Slaveuhr der Differenzwert bzw. die Uhrenabweichung berechnet.

Die Korrektur des Zeitstempels im Sendebereich des Masters erfolgt nach der im Abschnitt 4.4.2 Laufzeitermittlung im Sendepfad beschriebenen Methode. Auf der Empfangsseite des Slave-Modems wird die Laufzeitermittlung nach der im Abschnitt 4.4.3 Laufzeitermittlung im Empfangspfad beschriebenen Methode durchgeführt. Für den Sendezweig im Slave-Modem wird der gleiche Algorithmus eingesetzt wie im Sendezweig des Master-Modems. Das gleiche gilt für den Empfangszweig des Master-Modems, der verwendete Algorithmus entspricht dem Empfangszweig im Slave-Modem. Die Abbildung 4.17 zeigt noch einmal übersichtlich den Durchlauf des Zeitstempels durch die verschiedenen Sende- und Empfangsbereiche der Modems.
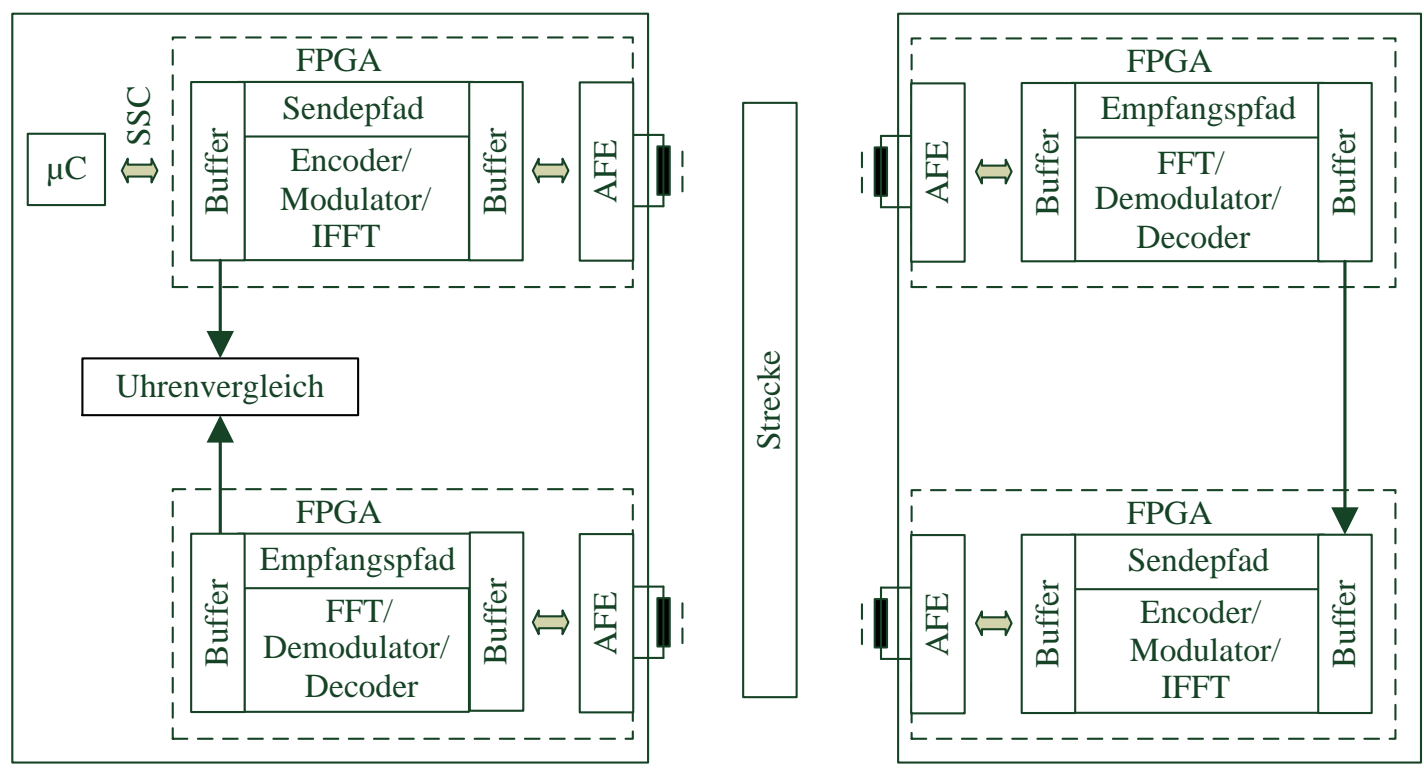

Abbildung 4.17.: Synchronisation zwischen Master- und Slave-Modem.

Die Messungen zur Ermittlung der Synchronisationsgenauigkeit wurden unter realen Bedingungen an der Testanlage (siehe Abschnitt 3.3) durchgeführt, d.h. Betrieb des Drehtisches und Energieversorgung der beweglichen Endgeräte über den Linienleiter. Bei dem Uhrenabgleich zwischen Master- und Slaveuhr wurden die Abweichun- 
gen der Uhren auf dem störungsbehafteten und bandbegrenzten Übertragungskanal innerhalb eines Toleranzbereiches von $\leq 30 \mu s$ ermittelt.

Bei der Ermittlung der Synchronisationsgenauigkeit wurde die eigentliche Laufzeit auf dem Übertragungskanal vernachlässigt bzw. nicht eingerechnet $(<1 \mu s)$. Um den Laufzeitfehler der Übertragungsstrecke zu kompensieren, kann das beschriebene Verfahren gemäß der im Abschnitt 4.1.1 beschriebenen Vorgehensweise erweitert werden. Dadurch können auch variable oder lange Laufzeiten, z.B. bei unterschiedlichen Entfernungen zwischen Master-Modem und mehreren Slave-Modems, auf dem Übertragungskanal berücksichtigt werden. Unterschiedliche Verarbeitungszeiten im FPGA bei Veränderung der Anzahl der zu übertragenden Prozessdaten sind im Konzept berücksichtigt.

Größere Synchronisationsintervalle reduzieren das Datenaufkommen pro Teilnehmer innerhalb eines betrachteten Zeitabschnittes, d.h. auch im Umkehrschluss, dass die Anzahl der Teilnehmer am Bus erhöht werden kann. Berücksichtigt werden muss dabei die Reaktionszeit im Fehlerfall eines Busteilnehmers (z.B. Umrichterstörungen, Schleppfehler, ..). Das gilt vor allem für Teilnehmer, die über eine gemeinsame Bewegungstrajektorie stark miteinander synchronisiert sind und deren Synchronisationszeitpunkte (z.B. Startpunkt der Bewegung oder Rampenverlauf) mit Hilfe von Zeitstempeln synchronisiert werden.

Der Vorteil eines Zeitstempelverfahrens gegenüber den Verfahren zur Synchronisation bei klassischen Bussystemen (vgl. Abschnitt 4.1.2) ergibt sich vor allem dadurch, dass keine permanente bzw. zyklische Synchronisierung zwischen den Teilnehmern erfolgen muss. Der Zeitstempel wird den Slaveteilnehmern eindeutig zugeordnet, hier ist auch eine asynchrone Datenübertragung im Sinne von variablen Synchronisationsintervallen bzw. Zeitfenstern möglich. Der Zeitstempel selbst kann dabei als Broadcast-Paket gleichzeitig an alle Teilnehmer versendet werden. Nach einer Anfangssynchronisierung an alle Teilnehmer (Übermittlung des aktuellen Uhrenstandes) muss im weiteren Verlauf der Datenübertragung nicht mehr zwingend der komplette Zeitstempel übertragen werden, sondern nur noch die maximale Abweichung der Uhr innerhalb des definierten Zeitintervalls der Synchronisation (z.B. nur Übergabe des $\mu s$-Zählers).

Zusätzlich kann zur Kontrolle und Zuordnung des Zeitstempels in der Slaveuhr, neben der obligatorischen Plausibilitätskontrolle des Zeitstempels selbst, auch noch die Übergabe einer fortlaufenden Sendezyklusnummer im Datentelegramm integriert werden.

Weitere Maßnahmen zur Verbesserung der Synchronität stellen die genannten Software- und Hardware-Optimierungen für die Ermittlung der Laufzeiten im Sende- und Empfangspfad entsprechend den Abschnitten 4.4.2 und 4.4.3 dar. Im Empfangspfad 
sollte es möglich sein, den exakten Erkennungszeitpunkt des empfangenen Chirpsignales noch genauer zu bestimmen und die Blockbearbeitung des FFT/IFFT-Blocks darauf zu synchronisieren. Im Sendepfad ist es möglich, durch die Realisierung einer synchronisierten Datenübertragung zwischen Mikrocontroller und FPGA über die SSC-Schnittstelle, weitere undefinierte Laufzeiten zu kompensieren.

Optimiert werden kann die Kommunikationsanbindung ferner durch das im Abschnitt 5.3.5 vorgestellte Konzept der Einbindung einer Soft-Core-CPU. Im realisierten Konzept kann durch die in sich geschlossene Lösung und Integration der Soft-Core-CPU die SSC-Schnittstelle entfallen.

In Kapitel 2 in Abbildung 2.2 wurde das Grundkonzept des intelligenten Aktors mit seinem strukturellem Aufbau vorgestellt. Dabei entsprechen die Abbildungen 4.7 und 4.8 den mit $K$ für Kommunikation bezeichneten Funktionsblöcken. Die in der Abbildung 2.2 mit $S$ für Synchronisation bezeichneten Funktionsblöcke (rot schraffierter Bereich) entsprechen den Abbildungen 4.15 und 4.16.

\subsubsection{Einfluss der Gangabweichung}

Werden Kommunikationsteilnehmer auf Basis vernetzter Uhren zueinander synchronisiert, ist auch zu berücksichtigen, welchen Einfluss die Gangabweichungen der einzelnen Oszillatoren untereinander auf die Genauigkeit des Synchronisationsvorgangs selbst haben. Die Frequenzstabilität oder auch Frequenztoleranz eines Oszillators wird üblicherweise in $p p m /{ }^{\circ} \mathrm{C}$ angegeben (vgl. Abschnitt 4.5).

Betrachtet man zwei Kommunikationsteilnehmer mit ihren taktgebenden Oszillatoren und unter Umständen verschiedenen Kennwerten für die Frequenztoleranzen der einzelnen Oszillatoren, so berechnet sich die maximale Gangabweichung der Teilnehmer bei einer bestimmten Temperatur und unter Berücksichtigung symmetrischer Frequenztoleranzen gemäß Gl. (4.17).

$$
F_{G \max }=\left(\left|T_{1 F t}\right|+\left|T_{2 F t}\right|\right) \Delta t_{S y n} .
$$

$F_{G m a x}$ beschreibt die maximale Gangabweichung bei zwei Teilnehmern. $T_{1 F t}$ die Frequenztoleranz des Oszillators über der Temperatur von Teilnehmer $1(\mathrm{ppm}), T_{2}$ Ft die Frequenztoleranz des Oszillators über Temperatur in ppm des Teilnehmers 2. $\Delta t_{\text {Syn }}$ beschreibt die Zeitdauer bzw. das Intervall der Synchronisation. Der auf der Modemplatine verwendete externe Oszillator (Abbildung 4.9) mit einem Systemtakt von $20 \mathrm{MHz}$ ist mit einer Frequenztoleranz von $50 \mathrm{ppm}$ innerhalb eines Temperaturbereiches von $-40^{\circ} \mathrm{C} \ldots+85^{\circ} \mathrm{C}$ angegeben (vgl. Abschnitt B.3). 
Für große Synchronisationsintervalle kann es durchaus zweckmäßig sein, Oszillatoren mit einer höheren Frequenzkonstanz zu verwenden. Dadurch wird die Gangabweichung der Oszillatoren untereinander reduziert. Für den konkret aufgebauten Versuchsaufbau beträgt das Synchronisationsintervall $20 \mathrm{~ms}$. Die verwendeten Oszillatoren besitzen eine Frequenzkonstanz von $50 \mathrm{ppm}$, d.h. der daraus resultierende maximale Fehler für die Gangabweichung liegt im Bereich kleiner $2 \mu s$.

Die Verwendung höherwertiger Oszillatoren mit besseren Kennwerten für die Frequenztoleranz ist daher bei langen Synchronisationsintervallen und gleichzeitig geforderter hoher Synchronisationsgenauigkeit verteilter Uhren zu berücksichtigen. Der Einfluss wird im nächsten Abschnitt näher betrachtet und untersucht.

\subsection{Bestimmung der Ganggenauigkeit mit der Zwei-Oszillator-Methode}

In Abschnitt 4.4.4 wurden Kommunikationsteilnehmer in einem Bussystem über lokal implementierte Uhren auf Basis eines Zeitstempelverfahrens synchronisiert. Dabei spielt die Bewertung der Ganggenauigkeit der verwendeten Uhren eine wichtige Rolle. Über die lokal implementierten Uhren in jedem Teilnehmer und Anwendung des Zeitstempelverfahrens ist es möglich, steuerungs- und regelungstechnische Abläufe zu koordinieren. Dabei ist es auch möglich, mehrere Teilnehmer bzw. Aktoren auf Basis hinterlegter Bewegungsprofile miteinander zu synchronisieren. Sinnvoll bzw. notwendig wird eine solche Vorgehensweise auch, wenn die Datenrate der Datenübertragung bedingt durch einen bandbegrenzten oder störbehafteten Übertragungskanal, wie bei dem aufgebauten Funktionsmuster mit induktiver kontaktloser Energieübertragung in Kapitel 3, eingeschränkt ist und gleichzeitig eine hohe Anforderung an die Synchronisation zwischen den Teilnehmern besteht.

Abhängig vom Synchronisationsintervall und der geforderten Synchronisationsgenauigkeit zwischen den Teilnehmern ist die Ganggenauigkeit der verwendeten Oszillatoren zu berücksichtigen. Für das Synchronisationsintervall bzw. den Synchronisationstakt stellen Oszillatoren eine zentrale Komponente dar. Sie fungieren als Taktgeber für die lokalen hochgenauen Uhren und bestimmen, in welchem Zeitraster eine Synchronisation der vernetzten Teilnehmer erfolgen muss, um vorgegebene Genauigkeitsanforderungen hinsichtlich der Ganggenauigkeit der Uhren zueinander gewährleisten zu können. Je besser die Ganggenauigkeit der Oszillatoren ist, desto größer kann das Synchronisationsintervall zwischen den lokalen Uhren gewählt werden.

Abschnitt B gibt einen einführenden Überblick über die Wirkungsweise und Eigenschaften von Oszillatoren. Die Vor- und Nachteile der Verwendung von Oszillatoren 
in technischen Systemen werden aufgezeigt. Konkret wird auf die in der Arbeit untersuchten unterschiedlichen Oszillatorklassen eingegangen. Weiterführende Informationen finden sich darüber hinaus in [77], [179].

Im Folgenden wird gezeigt, welche Eigenschaften und Abhängigkeiten bei den Oszillatoren in den verschiedenen Oszillatorklassen bestehen. In dem Versuchsaufbau wird dann empirisch auf Basis der Zwei-Oszillator-Methode untersucht, welche Genauigkeiten für die Gangabweichungen der verschiedenen Oszillatoren erreicht werden.

\subsubsection{Messaufbau zur Bestimmung der Gangabweichung}

Im folgenden Abschnitt werden unterschiedliche Oszillatoren empirisch auf ihre Ganggenauigkeit bzw. Gangabweichung hin untersucht. Ziel der Untersuchung ist nicht die absolute Bestimmung der Ganggenauigkeit eines einzelnen Oszillators, sondern die Ermittlung der relativen Ungenauigkeit zwischen zwei Oszillatoren. Werden zwei Oszillatoren miteinander verglichen, spricht man von der sogenannten Zwei-OszillatorMethode. Bei der Zwei-Oszillator-Methode dient ein erster Oszillator als Referenzoszillator, der mit einem zweiten Oszillator gleicher Oszillatorklasse verglichen wird.

Die Untersuchung erfolgt im Zeitbereich (vgl. Abschnitt B.2). Die Untersuchung ist möglich durch die Messung der Gangabweichung zweier Oszillatoren innerhalb eines definierten Zeitintervalls. Eine zweite Möglichkeit ist die Bewertung der Frequenzkonstanz durch die Bestimmung der Allan-Varianz für verschiedene Werte für $\tau_{0}$. Verglichen werden sollen hier ein XO-Oszillator, ein TCXO- und ein OCXO-Oszillator. Abbildung 4.18 zeigt den Aufbau für den TCXO- und den OCXO-Oszillator mit einem speziell dafür entworfenen Platinenlayout.
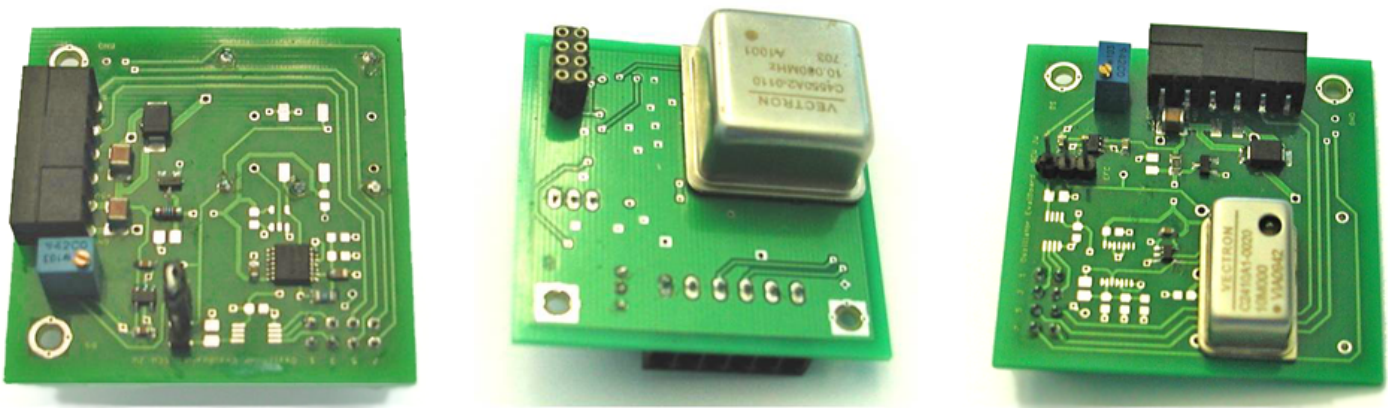

Abbildung 4.18.: links: OCXO-Platine (Oberseite), mitte: OCXO-Platine (Unterseite), rechts: TCXO-Platine.

Die Platinen enthalten alle notwendigen Bauelemente für die Spannungsversorgung, Bauteile für Pegelwandlungen und Pegelanpassungen sowie Möglichkeiten für den Signalabgriff. Ferner sind TCXO- und OCXO-Oszillatoren von Haus aus mit einem 
Justagepin ausgestattet, so dass ein fertigungsbedingter Frequenzoffset nachjustiert werden kann. Für die Bewertung der Ganggenauigkeit des Standard XO-Oszillators wurden für die Messungen die Taktausgänge der SMD-Oszillatoren auf der im Abschnitt 4.3.1 beschriebenen PLC-Modemplatine ausgewertet (vgl. Abbildung 4.9).

Der Takt eines jeden Oszillators wird von einer nachgeschalteten FPGA-basierten Testplatine ausgewertet. In jedem FPGA ist eine lokale Uhr bzw. eine Zähleinheit VHDL-basiert synthetisiert. Die entsprechende Taktung erfolgt durch den Oszillator. Abbildung 4.19 zeigt den prinzipiellen Aufbau.

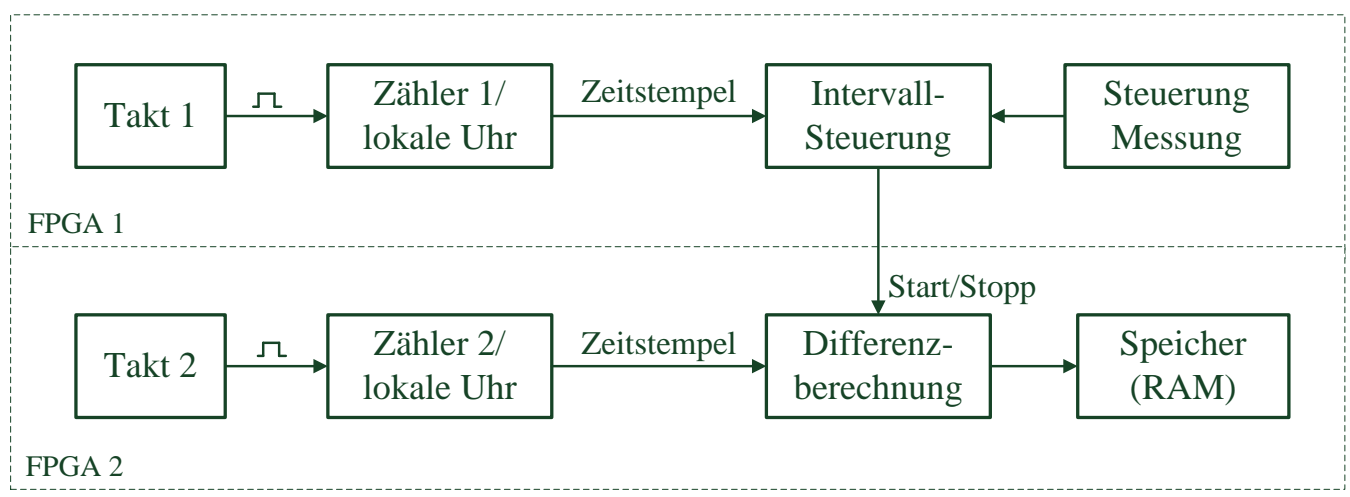

Abbildung 4.19.: Messaufbau zur Bestimmung der Gangabweichung.

Der Zeitstempel der lokalen Uhr setzt sich aus Mikrosekunden, der kleinsten internen Auflösung, weiter aus Millisekunden und Sekunden zusammen. Die Intervallsteuerung im FPGA 1 löst das Start- und Stoppsignal für die Differenzmessung im FPGA 2 aus. Die Kopplung ist mit einfachen binären Signalen (3,3 V TTL Pegel) realisiert. Das Modul Differenzberechnung zeichnet den aktuellen Zählerstand der lokalen Uhr 2 zum Start- und Stoppzeitpunkt auf und berechnet daraus die Abweichung zum Vorgabeintervall. Vorteilhaft bei der Verwendung einer FPGA-Plattform ist die hohe Verarbeitungsgeschwindigkeit im $n s$-Taktbereich des FPGAs sowie die Möglichkeit, parallele Abarbeitungsstrukturen aufzubauen, die sich nicht gegenseitig beeinflussen - im Gegensatz zur sequenziellen Signalverarbeitung bei Verwendung von Mikrocontrollern.

\subsubsection{Darstellung der Ergebnisse der Gangabweichung}

Die Basis der Synchronisationsgenauigkeit zwischen Masteruhr im Master-Modem und der Slaveuhr im Slave-Modem in den in Abschnitt 4.4.4 durchgeführten Messungen beruhen u.a. auch auf der Gangabweichung der Masteruhr und der Slaveuhr selbst. Bei schlechter Frequenzkonstanz der Uhren bzw. der von ihnen getakteten Oszillatoren driften die beiden Uhrenwerte immer stärker auseinander. 
Bei langen Synchronisationsintervallen wird der Effekt noch verstärkt. Neben der Synchronität verschlechtern sich zusätzlich die Gleichzeitigkeitseigenschaften (vgl. Abschnitt 4.1) zwischen Masteruhr und Slaveuhr. Werden die Busteilnehmer auf Basis vernetzter Uhren miteinander synchronisiert, ist zu berücksichtigen, welchen Einfluss die Gangabweichungen der einzelnen Oszillatoren untereinander haben.

Die dargestellten Messergebnisse der Gangabweichung basieren auf der im Abschnitt 4.5.1 beschrieben Zwei-Oszillator-Methode. Ein Oszillator dient als Referenzoszillator, ein zweiter Oszillator gleicher Oszillatorklasse als Vergleichsoszillator. Im folgenden wird für unterschiedliche Oszillatorklassen qualitativ gezeigt, nach welcher Zeitspanne eine kritische Grenze erreicht wird, nach der eine Synchronität der Uhren nicht mehr gegeben ist. Dabei ist die kritische Grenze in der Regel applikationsabhängig zu bewerten. In Abbildung 4.20, 4.21 und 4.22 sind die gemessenen Gangabweichungen der drei untersuchten Oszillatorpaare dargestellt.

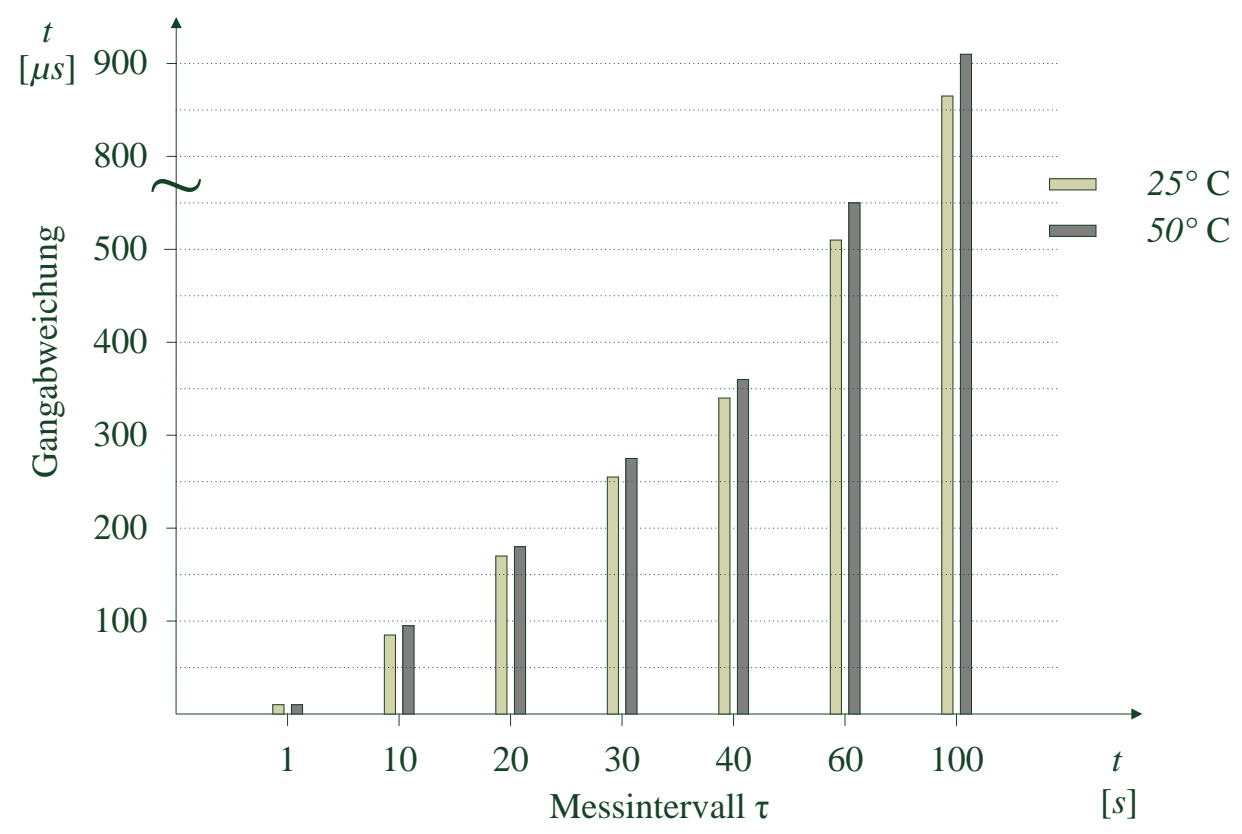

Abbildung 4.20.: Gangabweichung des Standard-Oszillators.

Auf der Ordinate ist die Gangabweichung in $\mu s$ aufgezeichnet, auf der Abszisse sind die unterschiedlichen Zeitintervalle, in dem die Messungen durchgeführt wurden, aufgetragen. Die Messungen sind bei zwei unterschiedlichen Temperaturen durchgeführt. Der linke Balken zeigt die Gangabweichung bei Zimmertemperatur $\left(25^{\circ} \mathrm{C}\right)$, der rechte Balken kennzeichnet die Messwerte für $50^{\circ} \mathrm{C}$ bei konstanter Ofentemperatur.

Der XO-Oszillator in Abbildung 4.20 zeigt als einziger Oszillator, im Vergleich zum TCXO-Ozillator in Abildung 4.21 und dem OCXO-Oszillator in Abbildung 4.22, bei Raumtemperatur bessere Werte für die Gangabweichung als bei $50{ }^{\circ} \mathrm{C}$. Ab dem Wert 
größer $1 s$ wird die Gangabweichung beim XO signifikant schlechter. TCXO- und OCXO-Oszillatoren zeigen erwartungsgemäß die weit besseren Werte. Auch das Verhalten bei der höheren Temperatur ist wie bereits angesprochen merklich besser.

Hier macht sich beim TCXO (vgl. Abbildung 4.21) der erhöhte schaltungstechnische Aufwand für die Temperaturkompensation bemerkbar sowie die Auslegung der Betriebstemperatur des Oszillators auf höhere Umgebungstemperaturen.

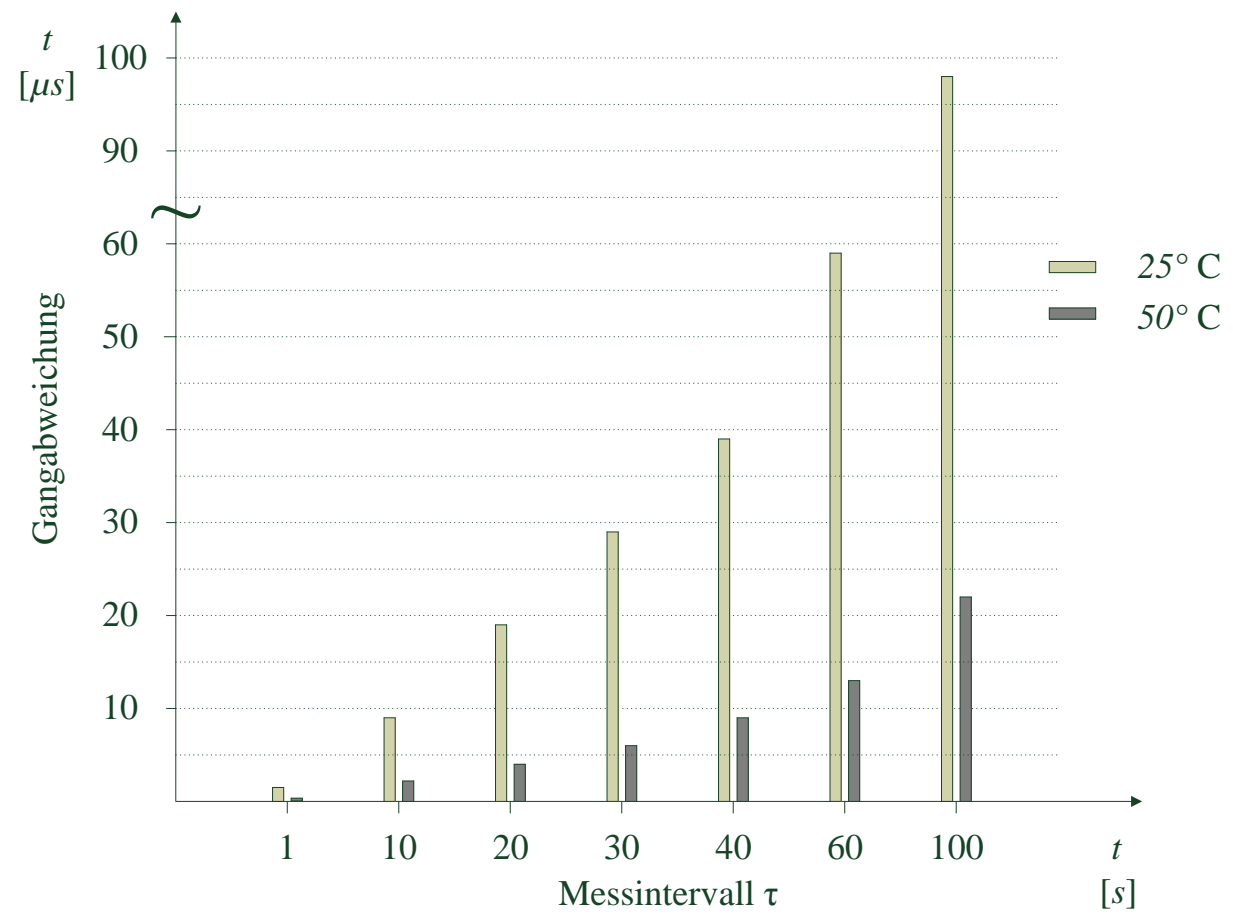

Abbildung 4.21.: Gangabweichung des TCXO-Oszillators.

Der OCXO-Oszillator in Abbildung 4.22 zeigt die besten Werte. Für ihn gilt ähnliches wie für den TCXO. Aufwändiger ist hier die integrierte Temperaturregelung des Oszillators sowie sein höherwertiger SC-Schnitt. Ein gutes Ergebnis liefert der OCXO-Oszillator für das Messintervall von $100 s$ und einer Umgebungstemperatur von $50^{\circ} \mathrm{C}$. Hier liegt die Gangabweichung bemerkenswert noch knapp unter $5 \mu s$.

\subsubsection{Bestimmung der Allan-Varianz}

Neben der im Abschnitt 4.5.2 beschriebenen ersten Methode die Gangabweichung eines Oszillators im Zeitbereich zu bestimmen, eignet sich als zweite Methode für die Bewertung der Frequenzkonstanz das Kriterium der Allan-Varianz. Die Allan-Varianz kennzeichnet in der Literatur ein Maß für die Stabilität von Oszillatoren und Uhren bzw. bewertet die Frequenzkonstanz innerhalb eines betrachteten Zeitraumes. 


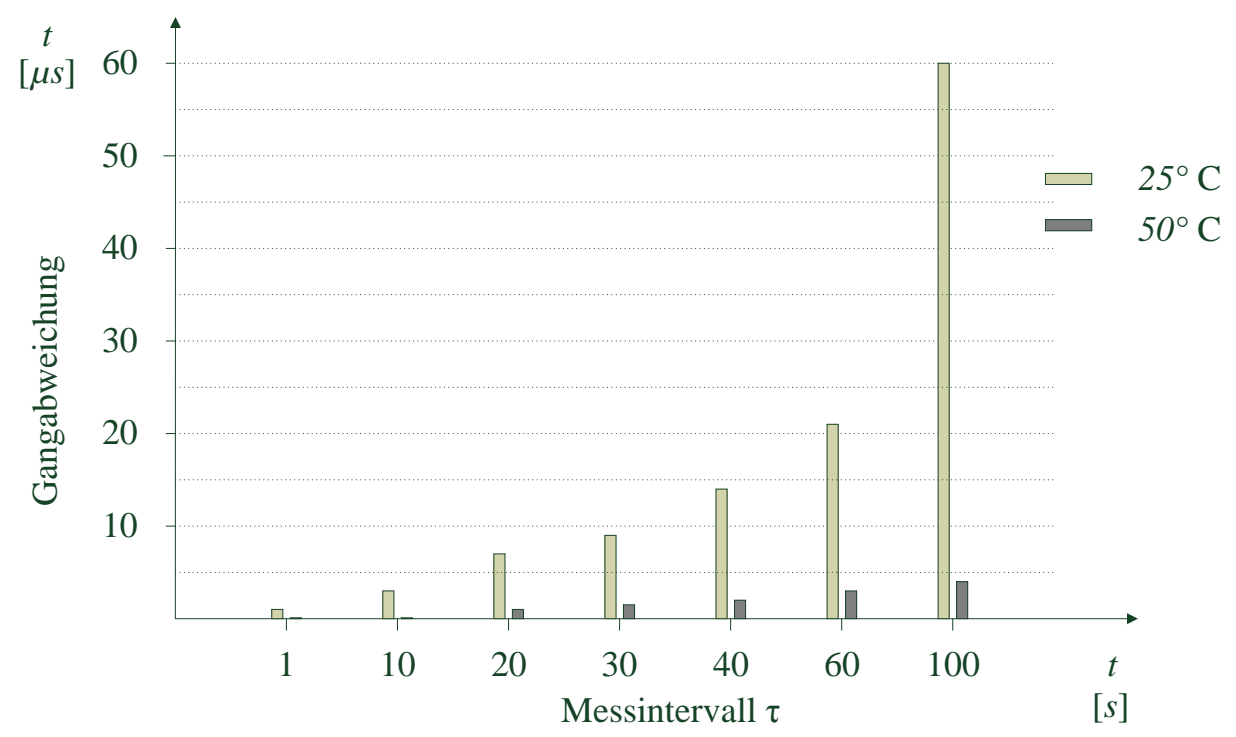

Abbildung 4.22.: Gangabweichung des OCXO-Oszillators.

Die Allan-Varianz ist eine Funktion des aufgenommenen Messintervalls als auch ihrer zugeordneten Verteilung (vgl. Abschnitt B.2), d.h. die Beschreibung erfolgt wie bei der ersten Methode in Abschnitt 4.5.2 im Zeitbereich. Die Darstellung der Varianz erfolgt in der Regel als Graph. Um reproduzierbare Ergebnisse zu gewinnen, sind mehrere Messungen innerhalb eines Zeitintervalls notwendig. Tabelle 4.3 zeigt die Anzahl der durchgeführten Messungen für jeden Intervallbereich von $\tau_{0}$.

Tabelle 4.3.: Messintervalle zur Bestimmung der Allan-Varianz

\begin{tabular}{llllllllllll}
\hline Messung & 1 & 2 & 3 & 4 & 5 & 6 & 7 & 8 & 9 & 10 & 11 \\
\hline Anzahl & 20 & 20 & 20 & 20 & 20 & 10 & 10 & 10 & 10 & 10 & 10 \\
\hline$\tau_{0}(s)$ & $10^{-4}$ & $10^{-3}$ & $10^{-2}$ & $10^{-1}$ & 1 & 10 & 20 & 30 & 40 & 60 & 100 \\
\hline
\end{tabular}

Die Messintervalle werden dabei sequenziell durchlaufen. Nach der gewünschten Anzahl wird automatisch auf das nächst größere Messintervall geschaltet. Nach jedem Ende eines Intervalls wird entsprechend von beiden Uhren der Zeitstempel ausgewertet. Das kleinste Zeitintervall für $\tau_{0}$ beginnt bei $100 \mu \mathrm{s}$ und endet bei $\tau_{0}=100 \mathrm{~s}$. Abbildung 4.23 zeigt die Ergebnisse der Messungen zur Bestimmung der Allan-Varianz für die verschiedenen Oszillatorklassen.

Die Werte für die unterschiedlichen Messintervalle sind entlang der Abszisse aufgetragen. Die Ordinate zeigt die Werte der zugehörigen berechneten Varianzen. Die Werte sind bei Zimmertemperatur $\left(25^{\circ} \mathrm{C}\right)$ bestimmt. Wie zu erwarten, zeigt der XOOszillator die schlechteste Frequenzkonstanz. Der TCXO-Oszillator zeigt ein besseres 


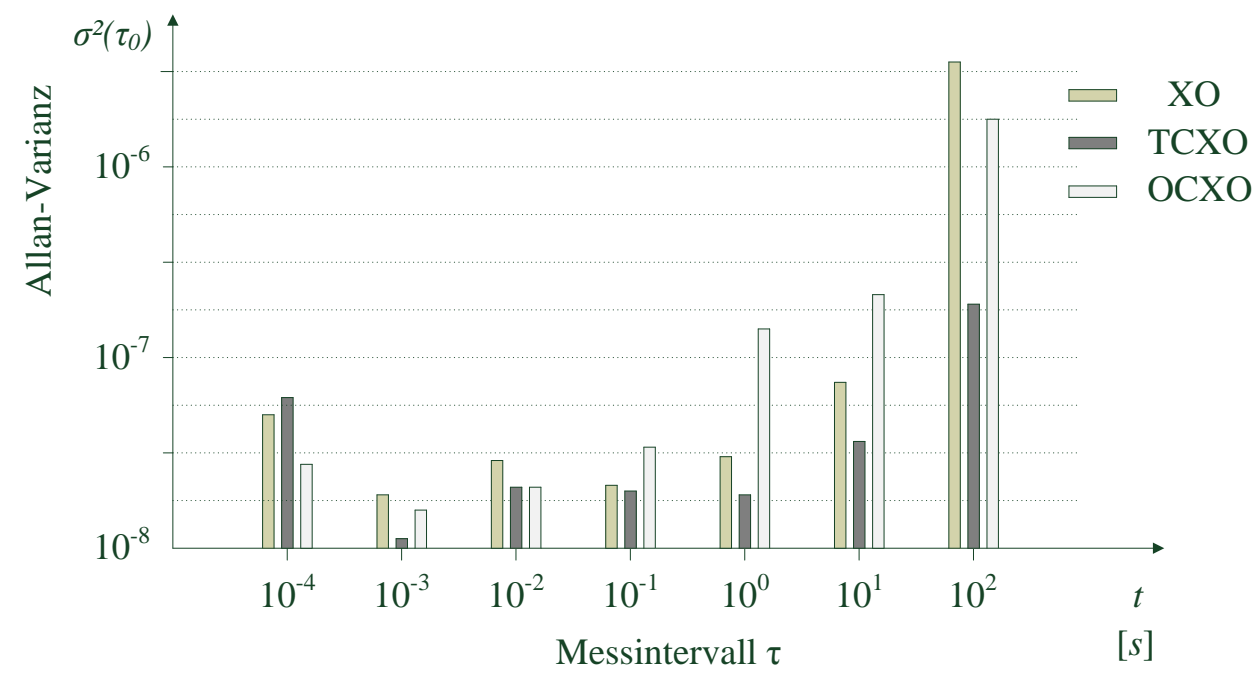

Abbildung 4.23.: Übersicht der Allan-Varianz aller drei Oszillatoren.

Verhalten, gerade ab dem Zeitintervall $>1 s$. Ab diesem Intervall driftet die Frequenz des einfachen XO-Oszillators stark ab.

Insgesamt zeigt sich der grundlegende theoretische „Badewannenverlauf“ des AllanVarianz-Graphen, wie in Abschnitt B.2 beschrieben, bestätigt. Die Ermittlung der Allan-Varianz für den OCXO-Oszillator ist allerdings nicht zufriedenstellend und spiegelt nicht seine hohe Frequenzkonstanz wider. Eine mögliche Erklärung ist die Verwendung eines Standard NAND-Gatters mit nicht konstanten Umschaltzeitpunkten bei der Signalpegel-Wandlung der Ausgangsfrequenz des OCXO-Oszillators. Die nicht konstanten Umschaltzeiten wirken in dem Fall wie eine zusätzliche Rauschquelle. Alternativ ist hier auch der Einsatz eines Komparators denkbar. Weitere Untersuchungen dahingehend wurden nicht durchgeführt.

\subsection{Zusammenfassung der Ergebnisse}

Am Beispiel des Versuchsaufbaus eines Funktionsmusters einer Etikettiermaschine wurde in Kapitel 4 gezeigt, dass durch die Wahl des OFDM-Übertragungsverfahrens und Auswahl geeigneter Frequenzkanäle eine Datenkommunikation zwischen stationärer Seite und den beweglichen elektrischen Verbrauchern über den induktiven kontaktlosen Energieübertragungskanal möglich ist.

Zu Beginn von Kapitel 4 wird auf die wichtigen Begriffe Synchronität und Gleichzeitigkeit in Automatisierungssystemen eingegangen. Das Prinzip auf Basis verteilter 
Uhren gemäß IEEE-1588 und die Merkmale eines modernen Feldbussystems werden erläutert. Das Referenzmodell des verwendeten Kommunikationssystems und der Aufbau des Datenübertragungssystems mit den zur Anwendung gekommenen digitalen Modulationsverfahren wurden dargestellt. Der Aufbau des PLC-Modems, die Einordnung in das OSI-Schichtenmodell sowie die notwendigen Funktionserweiterungen des Modems mit den wichtigsten erweiterten Registerfunktionen für die Datenübertragung wurden beschrieben. Der zentrale Bestandteil des Kommunikationsablaufes zwischen den stationären und beweglichen Teilnehmern wurde über die implementierte Master-Slave-Schnittstelle realisiert. Dabei wurden die ermittelten Parameter des Datenübertragungssystems bestimmt und in das Protokoll bzw. in den Kommunikationsablauf integriert. Die Anbindung zum Mikrocontroller wurde über die beschriebene SSC-Schnittstelle umgesetzt.

Im Abschnitt 4.4 wird unter realen Betriebsbedingungen des Funktionsmusters (vgl. Kapitel 3) nachgewiesen, dass sich durch eine Erweiterung des Datentelegramms und Integration des Zeitstempelverfahrens auch Kommunikationsteilnehmer miteinander effektiv synchronisieren lassen, die über den stark störungsbehafteten Übertragungskanal miteinander verbunden sind (vgl. Abschnitt 4.4.4). Dabei wurden die Durchlaufzeiten der Signale innerhalb der OFDM-basierten Datenkommunikation im Sende- und Empfangspfad des Modems durch die Nutzung paralleler Abarbeitungsalgorithmen im FPGA bestimmt. Es wurde gezeigt, wie sich die einzelnen Funktionsblöcke für die Kommunikation und die Synchronisation in das Gesamtkonzept des intelligenten Aktors in Kapitel 2 in Abbildung 2.2 einfügen.

Die Ganggenauigkeit von Oszillatoren spielt bei der Synchronisierung der Kommunikationsteilnehmer über Zeitstempel und für größere Synchronisationsintervalle eine wichtige Rolle. Daher wurde im weiteren Verlauf von Abschnitt 4.5 die Ganggenauigkeit unterschiedlicher Oszillatoren aus unterschiedlichen Oszillatorklassen untersucht. Auf Basis der Zwei-Oszillator-Methode konnte an einem praktischen Versuchsaufbau gezeigt werden, welche Ganggenauigkeiten bei verschiedenen Oszillatorklassen in Abhängigkeit unterschiedlicher Umgebungstemperatureinflüsse erreicht werden. Die Bewertung der Frequenzkonstanz bzw. die Allan-Varianz der unterschiedlichen Oszillatoren wurde bestimmt und der typische „Badewannenverlauf" nachgewiesen (vgl. Abbildung 4.23). Durch die Wahl höherwertiger Oszillatoren ist es möglich, die Synchronitätsgenauigkeit lokal implementierter Uhren markant zu verbessern bzw. das Synchronisationsintervall zwischen verschiedenen Teilnehmern merklich zu vergrößern.

Im nächsten Kapitel wird ein Konzept vorgestellt, wie ein kompakter intelligenter Aktor hardware- und softwaretechnisch aufgebaut werden kann, wobei die zu erarbeitenden Abläufe und Funktionsblöcke des Kapitels 5 in das Gesamtkonzept des intelligenten Aktors in Abbildung 2.2 eingebunden werden. 


\section{Neue Hardware- und Regelungsplattform für intelligente Aktoren}

Das in Kapitel 3 in Abbildung 3.11 aufgebaute dezentrale Antriebsmodul für bewegliche elektrische Verbraucher ist modular aufgebaut und setzt sich aus den einzelnen beschriebenen Komponenten wie Display, Antriebsumrichter, Steuerung, AnpassSteller, Verdrahtungsplatine und den Kommunikationsmodulen (PLC-Modem bzw. WLAN-Client) zusammen. Die dargestellte Variante stellt einen aktuellen Stand der Technik dar [203]. Weitere Hersteller von dezentraler Antriebstechnik sind z.B. [121], [145]. Insgesamt ist das verwendete Antriebsmodul allerdings zu groß und entspricht nicht den Anforderungen eines kompakten Aktors.

Nachfolgend wird ein neues Konzept eines kompakten intelligenten Aktors vorgestellt. Dabei wird der Ansatz verfolgt, die Komponenten des Kommunikationsmoduls (vgl. Kapitel 4) mit der Steuerung und dem Steuerteil des Antriebsumrichters zusammenzufassen und als intelligenten Aktor abzubilden. Eine mögliche Anwendung ist ein solcher intelligenter Aktor für die im Abschnitt 3.2 beschriebene Etikettieranlage.

Im Verlauf des Kapitels wird gezeigt, dass die vorhandene FPGA-Struktur im PLCModem (vgl. Kapitel 4) genutzt und erweitert werden kann, um ein kompaktes und skalierbares Motorsteuerkonzept für den Betrieb einer permanentmagneterregten Synchronmaschine (PMSM) innerhalb einer SoPC-basierten Plattform aufzubauen.

Zunächst werden im ersten Abschnitt von Kapitel 5 die Eigenschaften und die Unterschiede von Mikrocontrollern und FPGAs und ihrer Verwendung erläutert. Die Steuerplatine für die Motorregelung mit der dezentralen Leistungselektronik und der Aufbau und die Wirkungsweise der PMSM werden beschrieben. Alle wesentlichen mathematischen Zusammenhänge, die für die Umsetzung der FOC im FPGA notwendig sind, wie die Berechnungen für die Koordinatentransformationen, die Umsetzung der Strom- und Drehzahlregelung sowie die Realisierung der Raumzeiger- und Pulsweitenmodulation werden formuliert und dargestellt. 
In Abschnitt 5.3.5 wird dann gezeigt, wie die Umsetzung der FOC innerhalb einer SoPC-basierten Embedded System Plattform für die Ansteuerung einer PMSM realisiert ist.

Eine wichtige Untersuchung ist dabei die Phasenstrommessung des Drehstrommotors. Hier wird gezeigt, dass es durch den Einsatz moderner Strommessverfahren, wie Sigma-Delta-Wandler ( $\Sigma \Delta$-Wandler), möglich ist, die Strommesswerte für unterschiedliche Genauigkeitsanforderungen und Reaktionszeiten zu bestimmen.

\subsection{Mikrocontroller versus programmierbarer Hardware}

Die heutige schnelllebige Entwicklung im Bereich der Elektronik für neue Produkte und Gerätegenerationen stellt an die Entwickler immer größere Herausforderungen. Der Produktlebenszyklus, die Entwicklungskosten und die Entwicklungszeiträume, auch im Sinne von Time-to-Market $\left(\mathrm{TTM}^{1}\right)$, spielen eine entscheidende Rolle. Die Produkte sollen dabei innovativ, kostengünstig, kompakt und robust sein, das Entwicklungsrisiko so gering wie möglich.

Bei bestehenden Produkten spielt die Problematik der Abkündigung von aktuell verwendeten Bauteilen eine große Rolle. Aber auch bei veränderten Anforderungen oder Weiterentwicklungen einer Baugruppe ist ein Redesign der Baugruppe erforderlich. Die Flexibilität bzw. die Anpassungsfähigkeit der verwendeten Bauteile und Baugruppen spielt dabei eine wichtige und zentrale Rolle, um zeitsparend mit möglichst wenig Bauteilen die Baugruppe anzupassen, um so die gewünschten Anforderungen sicherzustellen.

In vielen klassischen elektronischen Anwendungen bildet ein Standard Mikroprozessor bzw. Mikrocontroller die Basis des Layouts. Der Mikrocontroller wird entsprechend der technischen Notwendigkeit ausgewählt. Viele Hersteller bieten für bestimmte Anforderungen - bei hohen Abnahmestückzahlen - zugeschnittene preiswerte Lösungen am Markt an. Schnittstellen für die notwendigen externen Anschlüsse an die Peripherie sind in der Regel bei solchen Controllern bereits im Design integriert.

Im Bereich der Antriebstechnik und im Motion-Control Umfeld werden am Markt eine Vielzahl von Controllerfamilien angeboten [211], [215]. Dabei kommen überwiegend Mikrocontroller oder auch digitale Signal-Controller (DSC), die DSP-Funktionalität im Controller integrieren, zum Einsatz. Die skalierbaren Controller sind hinsichtlich der benötigten Motion-Control-Anwendungen optimiert, um die erforderlichen Steuer-und Regelungsfunktionen zu erfüllen.

\footnotetext{
${ }^{1}$ gemeint: Vorlaufzeit, Produkteinführungszeit
} 
Die Kriterien für die Auswahl solcher Mikrocontroller sind u.a. die Taktfrequenz und die Auswahl geeigneter Speicher, wie z.B. eines FLASH-Speichers ${ }^{2}$ oder eines SRAM-Speichers (engl.: Static Random Access Memory). Weitere Auswahlkriterien sind die Anzahl der Timer, die Aufösung von A/D- und D/A-Wandler, die Anzahl der I/O-Punkte oder ob eine integrierte Pulsweitenmodulation verfügbar ist. Zusätzliche wichtige Merkmale können der Energiebedarf und die Betriebstemperatur des Bauteils sein und ob eine Anbindung an externe Schnittstellen wie CAN, SPI (engl.: Serial Peripheral Interface) oder $\mathrm{I}^{2} \mathrm{C}$ (engl.: Inter-Integrated Circuit) ermöglicht wird. Für die Programmierung der Motorregelung und für die Ansteuerung der Peripherie werden vom Hersteller oft Softwarebibliotheken zur Verfügung gestellt, teilweise auch Tools zur Steuerung und Überwachung des Motorbetriebes für den Testbetrieb während der Entwicklungsphase.

Die Entwicklung auf Basis eines klassischen Platinenlayouts birgt ein geringes Entwicklungsrisiko bei relativ kurzer Entwicklungszeit. Das Layout des Mikrocontrollers und seine bereitgestellten vorgeprüften Funktionalitäten und Schnittstellen sind einfach und schnell in das zu entwickelnde Schaltungslayout übertragbar. Der Nachteil ist, dass solche Standardkomponenten nur einen eingeschränkten Performance-Level abdecken - was meist auch aus Kostengründen gewünscht ist. Die Schnittstellen zur Peripherie sind wie bereits angesprochen integriert und auf die jeweiligen Erfordernisse zugeschnitten, dadurch sind sie aber auch herstellerabhängig.

Eine Alternative zum Mikrocontroller bietet der FPGA. Ein FPGA bietet dank seiner programmierbaren Hardware bzw. seinen frei programmierbaren Logikelementen vielfältige Möglichkeiten. Neben der großen Zahl von gleichartigen Logikgattern finden sich in heutigen modernen FPGAs auch parametrierbare Module bzw. Elemente wie z.B. Speicherbausteine, Multiplizierer oder PLLs, die sich im FPGA-Design schnell und bequem integrieren lassen. Zusätzliche Module oder eigentlich genauer gesagt "Theoretische Modelle“ können ergänzt werden, was zu komplexen digitalen Systemen führt. Die Module können grundsätzlich in sogenannte IP-Soft-Cores (eng.: Intellectual Property) und IP-Hard-Cores unterschieden werden [139].

Hard-Cores sind im Chip des FPGA-Hersteller als fertige Schaltung eingebunden. Gegenüber Soft-Cores sind Hard-Cores auf den FPGA speziell zugeschnitten, daher meist schneller in der Abarbeitung der Signalverarbeitung und belegen dabei gleichzeitig weniger Chipfläche. Soft-Cores werden im freiprogrammierbaren Teil eines FPGAs eingebunden, sie liegen in Form einer Netzliste oder als Quellcode vor. In der Literatur wird unter dem Begriff IP-Soft-Core oder auch nur Soft-Core eine für die jeweilige FPGA-Architektur zugeschnittene Prozessorkernstruktur verstanden.

Eingebundene Soft-Cores zur Anbindung der Peripherie oder anderer spezieller Funktionen - welche externe Hardware ersetzen können - werden allgemein als IP-Blöcke

${ }^{2}$ nichtflüchtiger digitaler Speicherbaustein 
bezeichnet, obwohl streng genommen, betreffend der physikalischen Umsetzung, kein Unterschied zur Implementierung zu einem Prozessorkern besteht.

Mittels FPGA, Soft-Core und den gewünschten peripheren IP-Blöcken, auch als IPCores bezeichnet, kann somit ein an die Applikation angepasstes Mikroprozessorsystem generiert werden. Die Vorteile eines solchen Systems sind, dass das Design für die Anforderung insgesamt sehr flexibel gestaltet werden kann. Spezielle IP-Blöcke können bisherige externe vorgeschaltete Chips in Hardware abbilden, was den Aufbau sehr kompakt und transparent macht.

Für die Umsetzung der Modelle im FPGA-Designs haben sich am Markt für die Simulation und Synthese die Hardwarebeschreibungssprachen VHDL [170] und Verilog [62] etabliert. Die zur Verfügung stehenden Modelle können so effizient und zeitsparend in das Design eingebunden werden. Rechenkapazität und Speichergröße lassen sich im FPGA anpassen. Nachträgliche Änderungen im Hardwaredesign sind schnell integrierbar und umsetzbar.

\subsection{Wahl einer geeigneten Referenzplattform}

Für die in Abschnitt 5.3 beschriebene umgesetzte FPGA-basierte Motorsteuerung wurde die nachfolgend beschriebene Referenzplattform gewählt. Bei der Referenzplattform handelt es sich um eine „Motion-Control“-Plattform der Fa. EBV Elektronik, die zusammen mit verschiedenen Halbleiterherstellern und der FH-Köln entwickelt wurde. Die genaue Bezeichnung der Plattform lautet FalconEye FPGA V1.4 [49]. Die Plattform beinhaltet den kompletten Leistungsteil eines Umrichters mit den notwendigen Funktionen und Peripheriebausteinen zur Ansteuerung eines bürstenlosen Wechselstrommotors (BLAC, engl.: BrushLess Alternating Current). In Zusammenhang mit der in Abschnitt 5.2.2 vorgestellten Steuerplatine ist der Betrieb einer permanentmagneterregten Synchronmaschine in der Betriebsart FOC möglich.

\subsubsection{Beschreibung der verwendeten Leistungsplatine}

Abbildung 5.1 zeigt das Blockdiagramm des modular aufgebauten Umrichters. Die Hauptmodule sind der Eingangsspannungsfilter, das PFC-Modul (engl.: Power Factor Correction) für die Korrektur des Leistungsfaktors, die IGBT-Endstufe, der Bremschopper für die Ansteuerung des Bremswiderstandes und die Schaltnetzteile.

Eingangsseitig kann das Leistungsmodul am einphasigen Stromnetz $\left(230 V_{A C}\right)$ betrieben werden. Die Zwischenkreisspannung wird auf $400 V_{D C}$ über das PFC-Modul geregelt. Durch die Korrektur des Leistungsfaktors werden Oberschwingungen reduziert, das Versorgungsnetz entlastet und man erhält einen nahezu sinusförmigen 


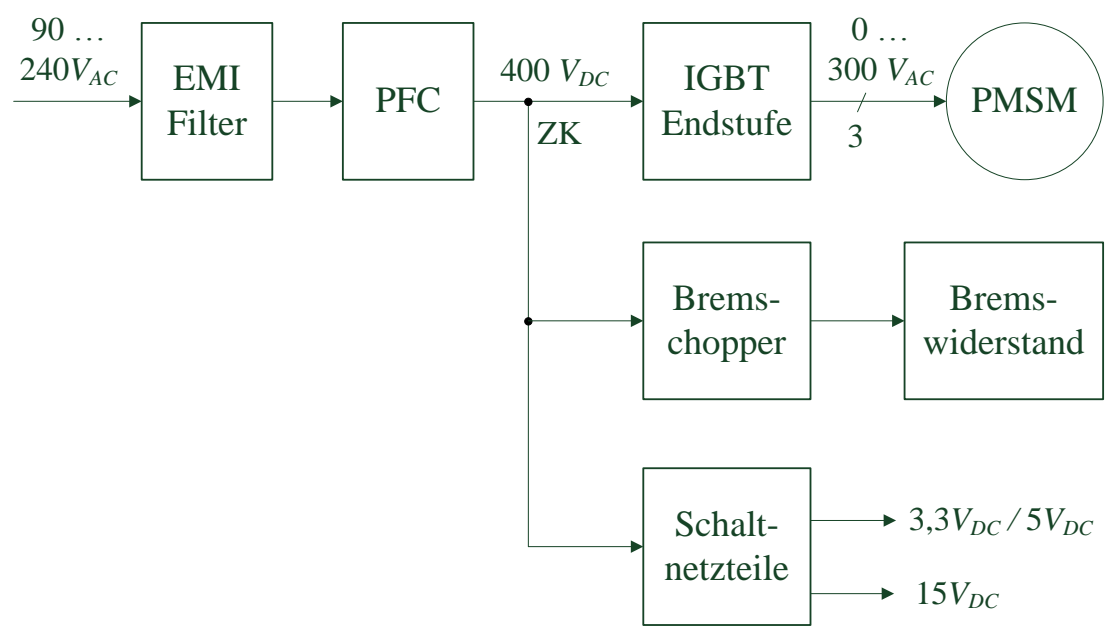

Abbildung 5.1.: Blockdiagramm für den Leistungsteil des Umrichters [49].

Netzstrom. Die Ausgangsspannung für die Motoransteuerung beträgt $300 V_{A C}$. Die Plattform ist auf eine Leistung von ca. $500 \mathrm{~W}$ ausgelegt.

Die Größe der Leistungsplatine beträgt in der Breite und Länge ca. $100 \mathrm{~mm}$ x $100 \mathrm{~mm}$. Durch die Verwendung hochintegrierter Leistungs- und Steuerelektronik ist ein kompakter Aufbau auf einer Leiterplatte möglich. Abbildung 5.2 zeigt den Aufbau der einzelnen Komponenten.

\section{PFC mit EMV-Filter}

Abbildung 5.3 zeigt den Schaltungsaufbau des Entstörfilters (EMI-Filter) und die Beschaltung des PFC-Chips (NCP1653, [147]) für die aktive Korrektur des Leistungsfaktors. Die CCM-PFC-Schaltung (engl.: Continous Conduction Mode) arbeitet nach dem Konstantspannungs-Verfahren mit einer festen Schaltfrequenz von $100 \mathrm{kHz}$. Bedingt durch die hohe Schaltfrequenz können die Induktivitäten zur Stromglättung kleiner und kostengünstiger dimensioniert werden. Ein Unter- und Überspannungsschutz ist bereits integriert.

Durch die Wahl des Widerstandes $R_{F B}$ können die gewünschte Zwischenkreisspannung und durch Wahl des Widerstandes $R_{\text {Sense }}$ der maximale Strom bzw. die maximale Leistung eingestellt werden. Für die äußere Beschaltung des PFC-Chips sind ein schneller $600 \mathrm{~V}$-Leistungsschalter (M1) und eine schnelle PFC-Diode (D1) [49] mit kurzer Sperr-Erholungszeit notwendig.

\section{Schaltnetzteil}

Beim Einschalten wird zunächst der Zwischenkreis des Umrichters über den Gleichrichter und den NTC (engl.: Negative Temperature Coefficient Thermistor) des PFCModuls geladen. Beim Überschreiten der Zwischenkreisspannung von etwa $80 \mathrm{~V}$ be- 


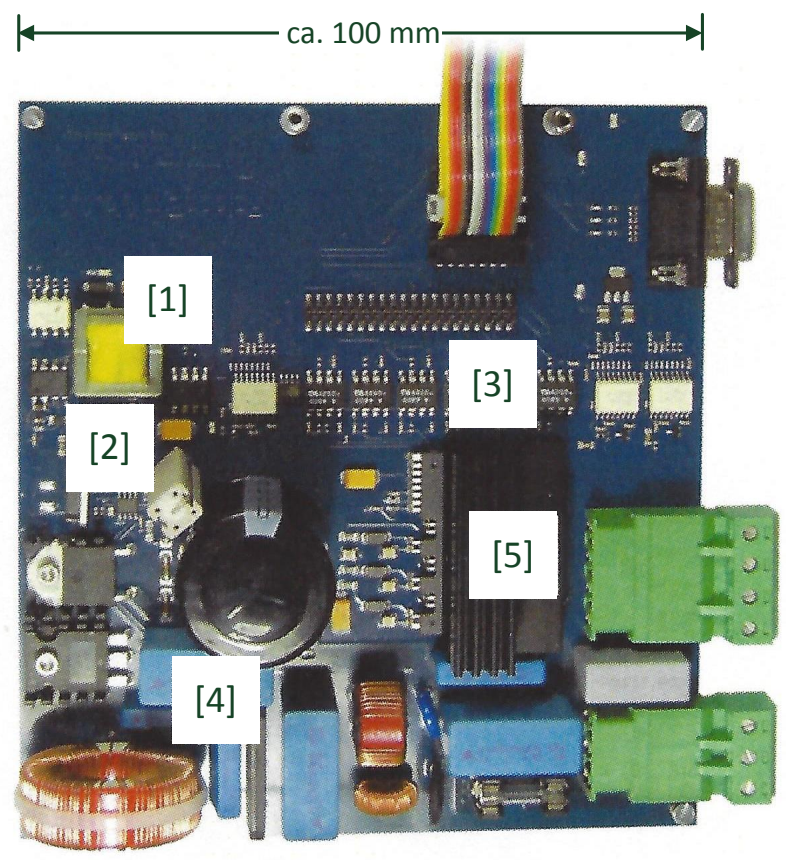

Abbildung 5.2.: Leistungsteil des Umrichters [49].

[1] Spannungsversorgung für die Logik mit Schaltnetzteilen, [2] Bremschopper, [3] Optokoppler und $\Sigma \Delta$-Modulatoren für die Strommessung, [4] PFC - Leistungsfaktor-Korrektur-Modul mit EMVFilter, [5] IGBT-Endstufe.

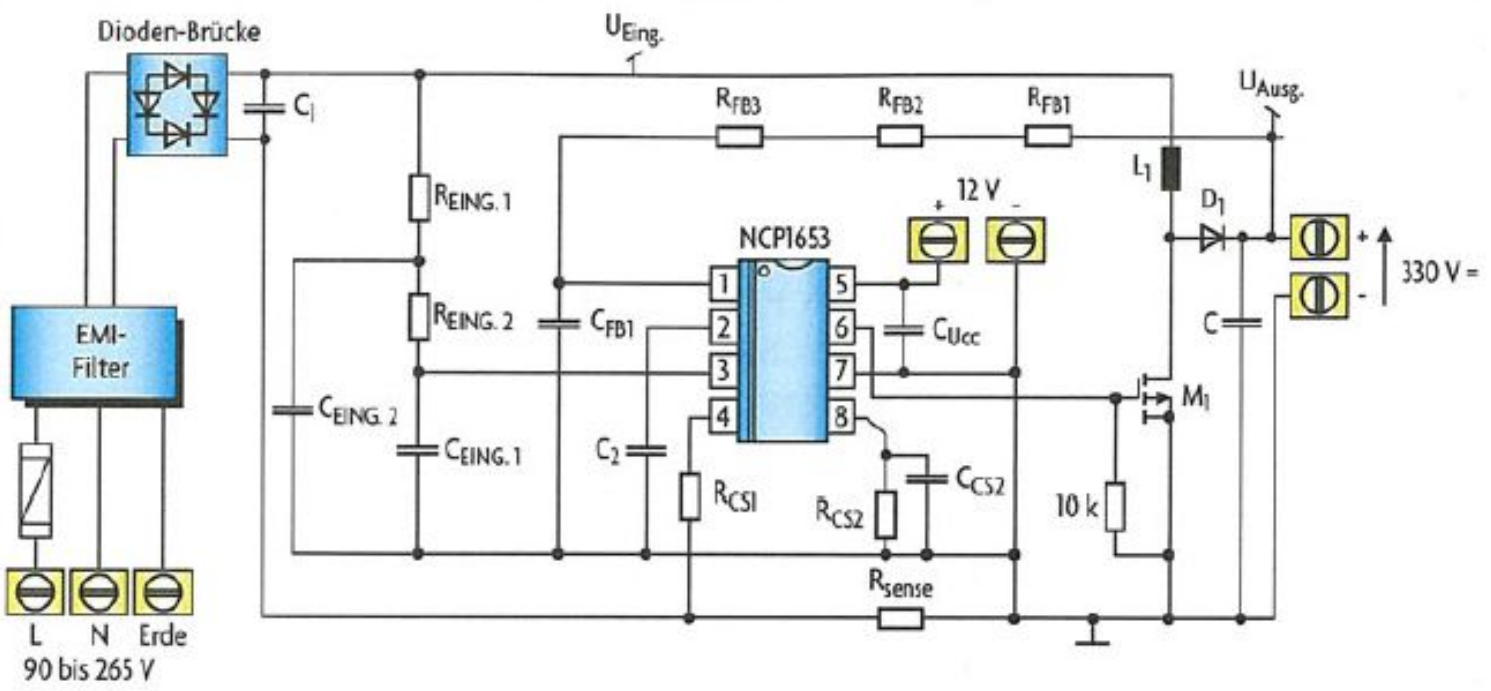

Abbildung 5.3.: PFC-Modul mit EMV-Filter [49]. 
ginnt das Schaltnetzteil (Abbildung 5.4) an zu schwingen. Am Ausgang des Netzteiles generiert der IC des Schaltnetzteiles [58] eine geregelte Spannung von $5 \mathrm{~V}$ für die Logikversorgung der Platine. Für die Versorgung der Gate-Ansteuerung wird eine ungeregelte Spannung von $15 \mathrm{~V}$ zur Verfügung gestellt. Beide Spannungen sind, wie Abbildung 5.4 zeigt, nach außen hin galvanisch getrennt.

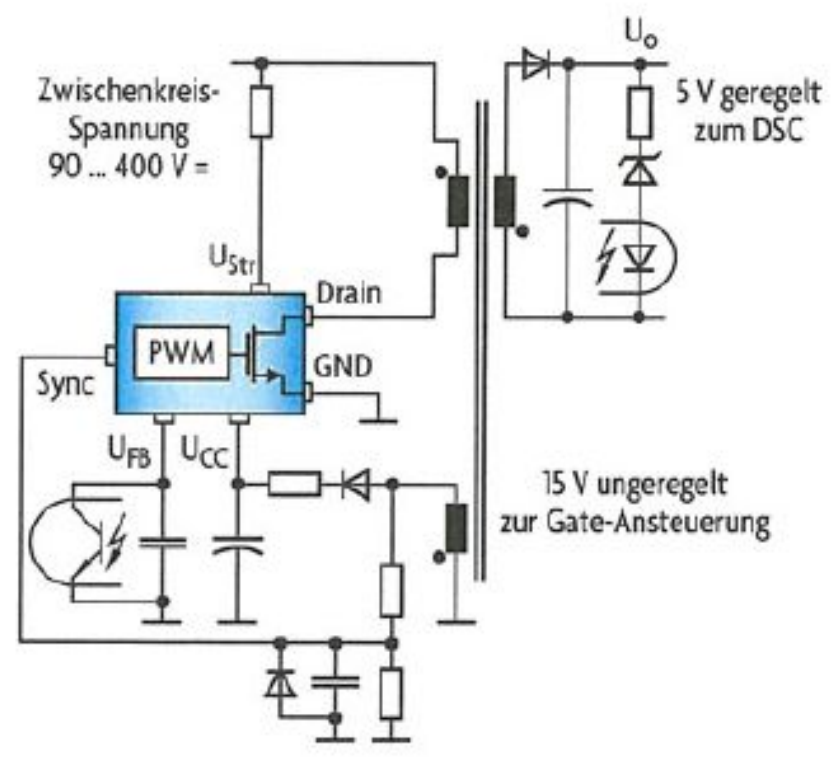

Abbildung 5.4.: Aufbau Schaltnetzteil [49].

\section{IGBT-Endstufe}

Die Ansteuerung des Motors erfolgt über ein „Smart Power"-Modul [59] wie in Abbildung 5.5 dargestellt. Bei dem Kompaktmodul handelt es sich um einen kompletten dreiphasigen IGBT-Inverter mit integrierter Gate-Ansteuerung und Schutzbeschaltung. Die optimierten Leistungsschalter zeichnen sich durch einen guten Wirkungsgrad bzw. durch eine geringe Verlustleistung aus. IGBT-Endstufen in der Leistungsklasse werden in hohen Stückzahlen hergestellt. Anwendungsgebiete sind z.B. die Ansteuerung kleinerer Pumpen in Haushaltsgeräten, wie in Spülmaschinen oder die elektronische Regelung drehzahlgeregelter Lüfter in Klimaanlagen. Auch industrielle Anwendungen sind möglich. Die drei Phasenströme können an Messwiderständen der herausgeführten Emittern der IGBT-Halbbrücken bestimmt werden. Zwei der drei Phasenströme ( $u$ - und $w$-Wicklung) werden abgegriffen, abgetastet und durch je einen $\Sigma \Delta$-Modulator 2. Ordnung [17] in Bitstream-Signale umgewandelt, welche direkt von der Steuerplatine ausgewertet werden.

Für die Gate-Spannungsversorgung der drei „High Side“-Transistoren wird die vorhandene $15 \mathrm{~V}$-Versorgungsspannung verwendet. Dabei wird bei geschlossener unterer IGBT-Halbbrücke über die Bootstrap-Diode ein Stützkondensator aufgeladen. Um 
die Steuerelektronik und die Leistungselektronik galvanisch zu trennen, sind für die Ansteuerung der Gatesignale sechs Optokoppler [16] auf der Leistungsplatine untergebracht. Eingangsseitig können so die 3,3 V-Steuerleitungen von der Steuerplatine bzw. vom FPGA-Board (Abschnitt 5.2.2) direkt verbunden werden. Auf der Ausgangsseite der Optokoppler werden über die $15 \mathrm{~V}$-Versorgungsspannung, welche auf dem Potentialniveau des Zwischenkreises liegt, die Gatesignale weiter auf das Leistungs-Modul geschaltet. Das Modul (max. $15 \mathrm{~A} / 600 \mathrm{~V}$ ) arbeitet dabei mit einer Zwischenkreisspannung zwischen $300 \mathrm{~V}$ und $400 \mathrm{~V}$.

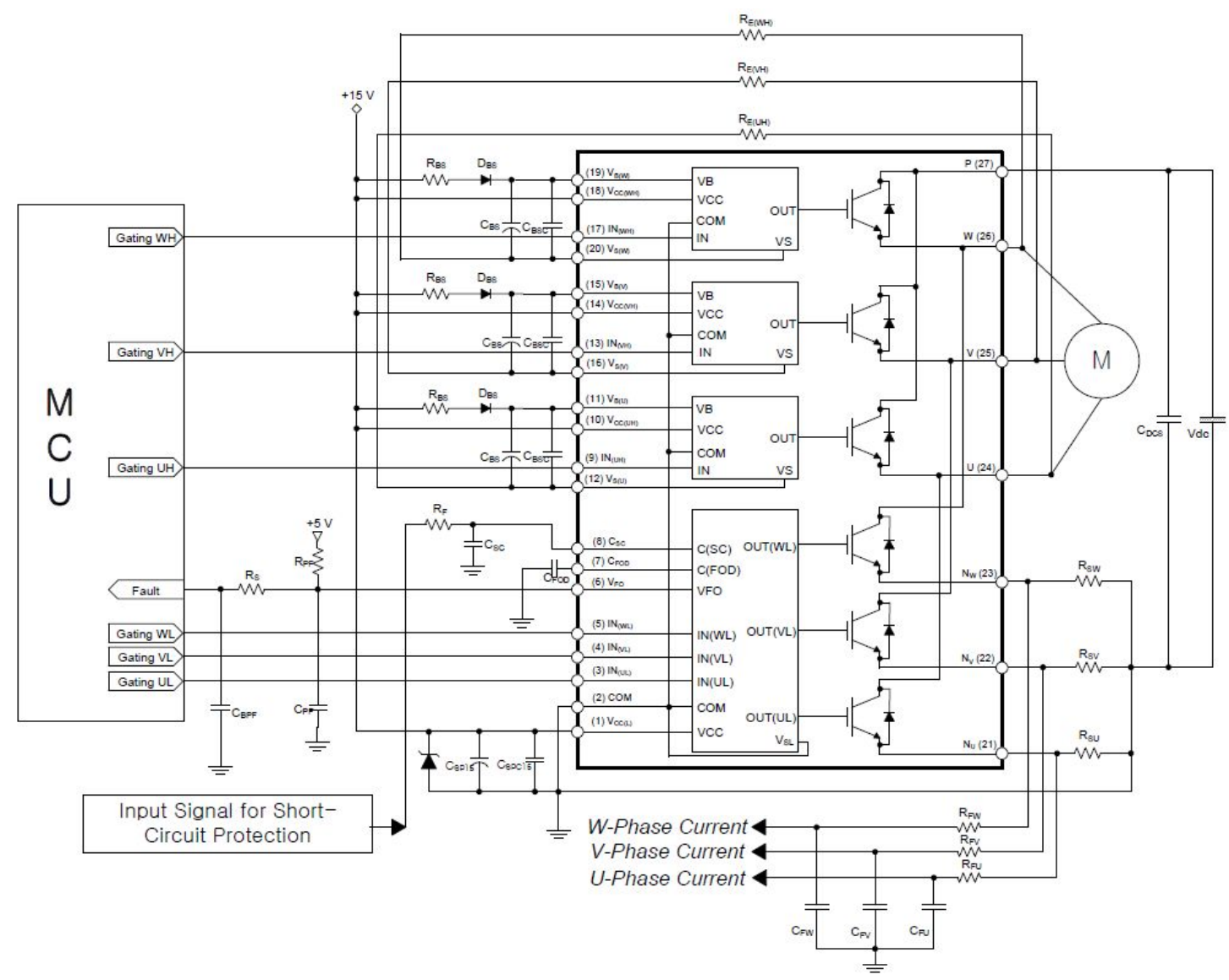

Abbildung 5.5.: Ansteuerung der IGBT-Endstufe [49].

\section{Bremschopper}

Um den Motor im Vierquadrantenbetrieb betreiben zu können, ist auf der Leistungsplatine ein Bremschopper integriert. Die gespeicherte Rotationsenergie $E_{\text {Rot }} \mathrm{im}$ Antrieb berechnet sich aus dem Trägheitsmoment des Antriebstranges $J$ und der aktuellen Drehzahl bzw. Winkelgeschwindigkeit $\omega$ des Motors, Gl. (5.1). 


$$
E_{R o t}=\frac{1}{2} J \omega^{2}
$$

Die im Zwischenkreis gespeicherte Energie im Umrichter $E_{Z K}$ bestimmt sich aus der Kapazität $C$ des Zwischenkreiskondensators und der aktuellen Zwischenkreisspannung $U_{Z K}$, Gl. (5.2).

$$
E_{Z K}=\frac{1}{2} C U_{Z K}^{2}
$$

Wird der Motor über die Leistungselektronik dynamisch gebremst, steigt die Spannung im Zwischenkreis je nach Anwendungsfall mehr oder weniger stark an. Besteht keine Rückspeisemöglichkeit ins Stromnetz, muss bei Überschreiten der Zwischenkreisspannung über einen kritischen Spannungswert die elektrische Energie im Zwischenkreis über einen Ballast-Widerstand in thermische Energie umgewandelt werden.

Der Wert der Zwischenkreisspannung wird über einen $\Sigma \Delta$-Modulator abgegriffen und über ein Bitstream-Signal direkt an die Steuerplatine übertragen. Als Schalter für das Zuschalten des Bremswiderstandes fungiert ein IGBT, der über einen Gate-Treiber angesteuert wird. Das aktive Ansteuersignal des Gate-Treibers liefert wieder die Steuerplatine. Der gewählte Ballastwiderstand beträgt $100 \Omega$. Die kurzzeitige Leistung $P$ berechnet sich dann nach Gl. (5.3). Die Dauerleistung ist auf $10 \mathrm{~W}$ ausgelegt.

$$
P_{\text {max }}=\frac{U^{2} Z K}{R}=\frac{(400 \mathrm{~V})^{2}}{100 \Omega}=1,6 \mathrm{~kW} .
$$

\subsubsection{Beschreibung der verwendeten Steuerplatine}

Als Steuerplatine für die Ansteuerung des Leistungsteils dient das Development Board DBC3C40-V1.1 der Firma EBV Elektronik [48]. Das Board ist optimiert für vielfältige Anwendungen im industriellen Umfeld. Zahlreiche Bausteine, Schnittstellen und Funktionen sind auf der Platine untergebracht. Der Kern der Platine bildet der EP3C40F484C7N, ein Cyclone III FPGA der Fa. Altera, der insgesamt 39.600 logische Elemente, 141 Kbyte RAM, 126 Multiplizierer (18 - 18 Bit) und 4 PLLs zur Verfügung stellt [5]. Mit den vier PLLs lassen sich synchrone und wahlweise phasenverschobene Frequenzen erzeugen. Das Board wird dabei über einen externen Quarz-Oszillator mit einem Systemtakt von max. $50 \mathrm{MHz}$ getaktet. Abbildung 5.6 gibt einen Überblick über den Aufbau des Boards. Das Maße der Platine betragen in der Breite und Länge ca. $100 \mathrm{~mm}$ x $100 \mathrm{~mm}$. 


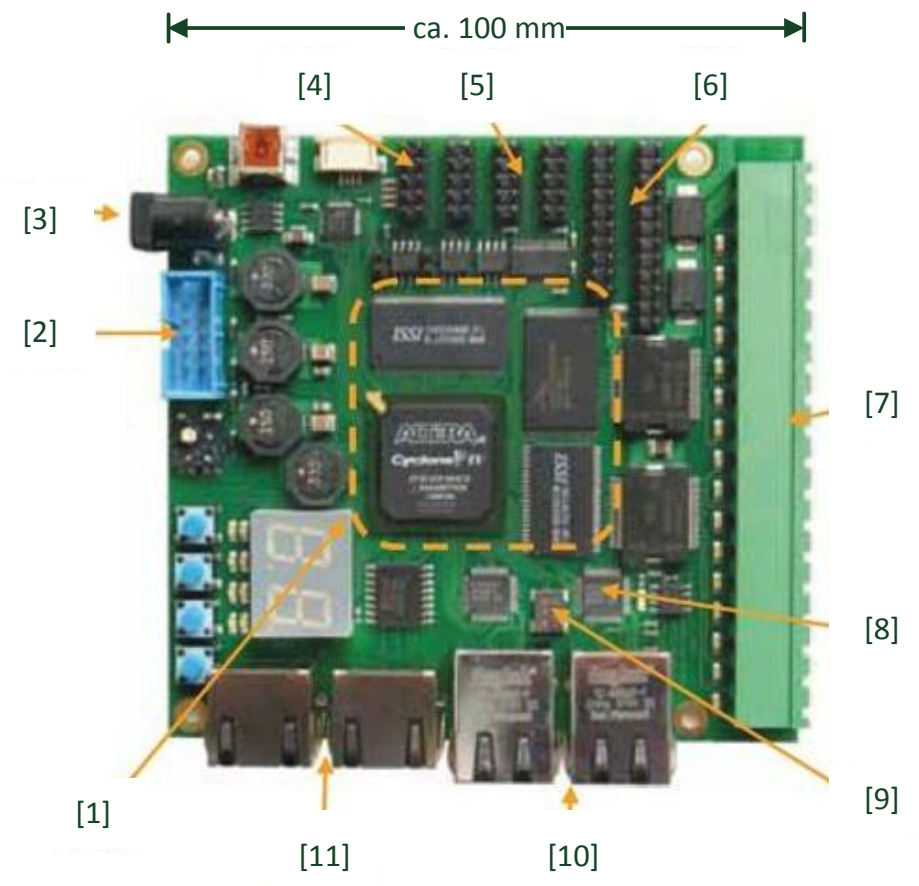

Abbildung 5.6.: Steuerteil Umrichter [48].

[1] EP3C40F484C7-FPGA mit SRAM, Flash und SDRAM, [2] Programmieranschluss, JTAG-Schnittstelle, [3] Stromversorgung, Steckeranschluss für externes Netzteil, [4] CAN-Kommunikation, [5] UART und RS485 Anschluss, [6] 3,3 V I/O-Pins, [7] $24 \mathrm{~V} \mathrm{I/O-}$ Klemmleiste mit LED, [8] Ethernet PHY, [9] externer $50 \mathrm{MHz}$ Quarz-Oszillator, [10] 2 x RJ45 Ethernet-Anschluss, [11] 2 x RJ45 LVDS-Anschluss.

\section{Externer Speicher}

Das Board bietet 1 Mbyte SRAM (512 K $\cdot 16$ Bit), 8 Mbyte Flash $(4 M \cdot 16$ Bit) und 16 Mbyte SDRAM (4 M.32 Bit). Der Flash-Speicher dient vor allem als ROM für den Soft-Core-Prozessor (NIOS II). Die Breite des Datenbusses beträgt 16-Bit. Ein 32Bit-Zugriff wird dabei in zwei 16-Bit-Zugriffe aufgeteilt. Zur Speicherung der FPGAKonfiguration steht ein serieller 64 Mbit Flash-Chip zur Verfügung [10]. Ein Teil des internen Speichers des FPGAs kann nach der Initialisierung aus dem Konfigurationsbaustein als ROM verwendet werden. Weiterhin befindet sich auf dem Board ein serielles EEPROM, das für den Versuchsaufbau aber nicht benötigt wird. 


\section{Kommunikation}

Für Kommunikationsaufgaben steht u.a. ein UART Transceiver [133] für die RS232Schnittstelle zur Verfügung. Weiterhin sind vier RS485 Transceiver [214], zwei CAN Transceiver [213] und ein USB 2.0 OTG Interface (engl.: On-the-go) vorhanden. Die Ethernet-Schnittstelle auf dem Board unterstützt zwei 10/100 Mbit PHYs [212]. Zwei weitere LVDS Ports (engl.: Low Voltage Differential Signaling) bieten zusätzlich die Möglichkeit, eine schnelle Datenübertragung zu realisieren.

\section{Ein- und Ausgänge}

Zur Anbindung an eine externe Steuerung können 16 binäre $24 V_{D C}$ Signale an der Klemmenleiste abgegriffen werden und individuell als Eingangs- oder Ausgangssignal parametriert werden. Weitere 32 I/O Pins sind direkt mit dem FPGA verbunden (3,3 V-Pegel). Die Signale werden für die Ansteuerung der Leistungsplatine des Umrichters verwendet. Dabei wird über ein Flachbandkabel eine direkte Verbindung über die Stiftleiste, die auf der Platine aufgelötet ist, hergestellt.

\section{Benutzerschnittstellen}

$\mathrm{Zu}$ Testzwecken stellt das Development Board vier Bedientaster, acht LEDs und eine 7-Segment Anzeige auf der Platine bereit. Zum Downloaden von Programmen, zum Testen und Debuggen steht eine JTAG-Schnittstelle [48] zur Verfügung.

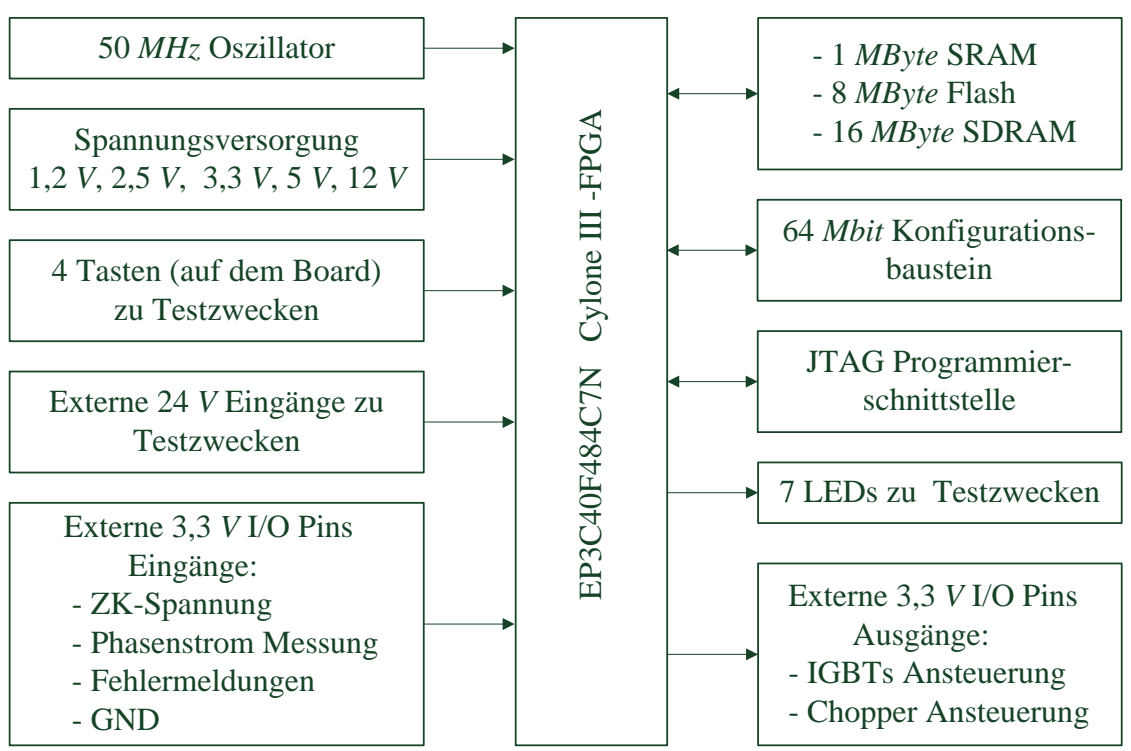

Abbildung 5.7.: Blockdiagramm DBC3C40 Development Board [48].

Ferner besteht die Möglichkeit, ein LVDS basiertes TFT-Interface (engl: Thin-Film Transistor) anzuschließen. Ein Navigation Key kann bei Anschluss eines TFT Dis- 
plays für Visualisierungsaufgaben genutzt werden. Abbildung 5.7 zeigt das Blockdiagramm des Boards mit den für den Versuchsaufbau verwendeten Bausteinen und Komponenten.

\subsection{Darstellung der neuen Hardware- und Regelungsplattform}

Die nachfolgenden Abschnitte beschreiben den Aufbau und die Entwicklung einer FPGA-basierten Motorsteuerung zur Ansteuerung einer PMSM, wie sie in typischen Servo-Anwendungen zum Einsatz kommt. Zuerst wird kurz auf den Aufbau und die Ansteuerung der PMSM eingegangen. Im Anschluss sind alle grundlegenden Bestandteile und wesentliche mathematische Zusammenhänge beschrieben, die für die Umsetzung einer feldorientierten Regelung auf der FPGA-Plattform notwendig sind.

\subsubsection{Permanentmagneterregte Synchronmaschine}

Permanentmagneterregte Synchronmaschinen (PMSM) sind weltweit im industriellen Umfeld verbreitet, besonders im unteren Leistungsbereich der Servoanwendungen. Die PMSM unterscheidet sich im Vergleich zu einer klassischen Synchronmaschine (SM) im wesentlichen durch den unterschiedlichen Rotoraufbau. Während eine herkömmliche SM auf eine Erregerwicklung im Rotor angewiesen ist, erfolgt die Erregung bei der PMSM durch Permanentmagnete (PM). Für niedrige Drehzahlen werden die Magnete entweder auf den Rotor geklebt (Surface PM, SPM) oder in den Rotor eingelassen (Surface inset PM, SIPM). Bei höheren Drehzahlen werden die Magnete mechanisch fixiert und mit einer Umhüllung gesichert [181].

Durch die Verwendung der PM und den Wegfall der Wicklung wird der Rotoraufbau leichter und kompakter. Bedingt durch das geringere Rotorträgheitsmoment entsteht dabei ein sehr gutes dynamisches Verhalten des Antriebes. Insgesamt führt das zu einer kleineren Bauform und einer höheren Leistungsdichte als bei vergleichbaren Asynchronmaschinen (ASM). Im Rotor entstehen durch die Verwendung der PM keine Stromwärmeverluste, eine Versorgung der Erregerwicklung über die Schleifringe bzw. über den Kommutator entfällt zusätzlich. Auch die Kühlung der Maschine (Stator) vereinfacht sich.

Nachteilig sind unter Umständen die höheren Kosten. Die verwendeten Magnetmaterialen zeichnen sich durch eine hohe Remanenzinduktion $\left(B_{r}=0,8 \ldots 1,3 T\right)$ aus. Materialien wie Neodymium-Eisen-Bor (NdFeB) oder Samarium-Kobalt (SmCo)Magneten aus der Gruppe der Seltenen-Erden sind teuer und unterlagen in den letzten Jahren starken Preisschwankungen am Markt. Als einen weiteren Nachteil 
kann ferner die schlechte Veränderbarkeit des Erregerfeldes genannt werden. Da viele Anwendungen der PMSM durch die oben genannten Vorteile im klassischen Servobereich zum Einsatz kommen, ist der Umstand in der Regel aber von untergeordneter Bedeutung.

\section{Blockkommutierung und Sinuskommutierung}

Bei der Ansteuerung von PMSM unterscheidet man im wesentlichem zwischen zwei unterschiedlichen Verfahren. Bei der blockkommutierten Maschine, auch als Elektronikmotor, elektronisch kommutierter Motor, BLDC (engl.: Brushless Direct Current) oder auch permanenterregter bürstenloser DC-Motor bezeichnet, wird immer gleichzeitig in zwei Phasen ein blockförmiger Strom eingeprägt. In Abhängigkeit von der Rotorposition (einfacher Rotorgeber aus Hallsensoren [159], [181]) wird zwischen den Phasen umgeschaltet, was auch bauartbedingt durch die Anordnung von konzentrierten Wicklungen im Stator eine rechteckförmige Verteilung der Luftspaltinduktion zur Folge hat. Bedingt durch die Abweichungen der Ströme bzw. der indizierten Spannung vom idealen rechteck- bzw. trapezförmigen Verlauf treten Drehmomentschwankungen auf, was die Gleichlauffähigkeit der Maschine einschränkt [42].

Bei der sinuskommutierten Maschine werden gleichzeitig alle drei Phasen der Motorwicklung bestromt. Statorstrom, Polradspannung und Flussdichte zeigen einen sinusförmigen Verlauf. Das bewirkt ein umlaufendes Magnetfeld entlang des Luftspalts wie bei einer klassischen Drehfeldmaschine. Die Bildung einer sinusförmigen Induktionsverteilung im Luftspalt wird bauartbedingt unterstützt durch sinusförmig verteilte Wicklungen im Stator oder durch eine entsprechende Anordnung der Magnete.

Sinuskommutierte PMSM zeichnen sich durch Abgabe eines konstanten Drehmomentes mit niedrigem Oberwellengehalt aus und erlauben auch die Anwendung der feldorientierten Regelung. Für solche Anwendungen ist der Motor mit einem höheraufösenden Gebersystem ausgestattet, wie Resolver oder einem digitalen Geber.

\section{Spannungsgleichungen der permanentmagneterregten Synchronmaschine}

Der prinzipielle Aufbau einer zweipoligen PMSM ist in Abbildung $5.8 \mathrm{zu}$ sehen. Die drei konzentrisch angebrachten Spulen des Stators sind jeweils um $120^{\circ}$ versetzt die Permanentmagnete sind entlang des Rotors angebracht bzw. aufgeklebt.

Durch die Steuerung der Phasenströme $i_{a}, i_{b}, i_{c}$ entsteht entlang des Luftspaltes ein resultierendes rotierendes magnetisches Feld, das den Rotor antreibt. Steht das Statorfeld genau $90^{\circ}$ zum Feld des Permanentmagneten kann das maximale Drehmoment übertragen werden (ziehende und abstoßende Wirkung der beiden Magnetfelder 


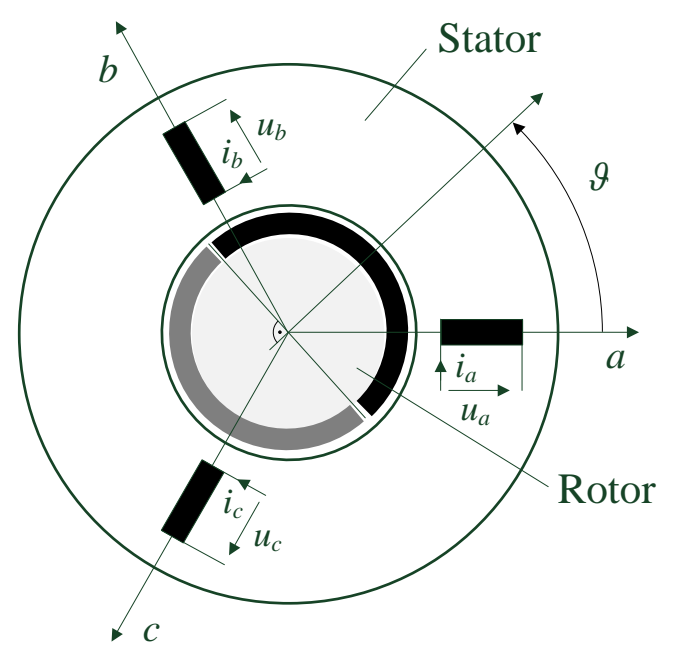

Abbildung 5.8.: Modell der PMSM mit Oberflächenmagneten [190].

zueinander). Die Spannung an jeder Spule ergibt sich aus dem Ohmschen Spannungsabfall an der Spule sowie der induzierten Spannung aus der Induktivität $L_{s}$ des Spulenstromes. Der Betrag des Rotorflusses $\psi_{P M}$ des Permanentmagneten ist dabei konstant, der Einfluss der induzierten Spannung an jeder Statorspule ist abhängig von der Rotorposition, Gl. (5.4) und Gl. (5.5) [186], [190].

$$
\begin{aligned}
u & =R_{s} i+\frac{d}{d t} \psi \\
\psi & =L_{s} i+\psi_{P M} \cos \vartheta .
\end{aligned}
$$

Für den eingeschwungenen Zustand, ( $\omega=d \vartheta / d t=$ konstant) und unter Berücksichtigung der geometrischen Anordnung der Spulen zueinander (hier 120²), können die Spannungsgleichungen für jede Phase angegeben werden, Gln. (5.6), (5.7), (5.8). Zweckmäßigerweise wird die erste Spule auf die $0^{\circ}$ Position festgelegt bzw. auf die die $a$-Achse bezogen [181], [190].

$$
\begin{aligned}
& u_{a}=R_{s} i_{a}+L_{s} \frac{d}{d t} i_{a}-\psi_{P M} \omega \cos \vartheta \\
& u_{b}=R_{s} i_{b}+L_{s} \frac{d}{d t} i_{b}-\psi_{P M} \omega \cos \left(\vartheta+\frac{2 \pi}{3}\right) \\
& u_{c}=R_{s} i_{c}+L_{s} \frac{d}{d t} i_{c}-\psi_{P M} \omega \cos \left(\vartheta+\frac{4 \pi}{3}\right) .
\end{aligned}
$$




\subsubsection{Koordinatentransformationen}

Die Darstellung der Gesamtdurchflutung bzw. magnetischen Flussdichten von dreiphasigen Wicklungssystemen im Stator von Drehfeldmaschinen wird üblicherweise durch Raumzeiger beschrieben [108], [191]. Raumzeiger lassen sich generell in ihrer Grundform auf die Behandlung von drei-Größen-Systemen zuschneiden, in denen die Summe-Null-Bedingung der einzelnen Komponenten $k a+k b+k c=0$ immer erfüllt ist.

Da bei der in Stern oder in Dreieck geschalteten Drehfeldmaschine allgemein von den drei Größen nur zwei Größen (Ströme, Spannungen, magnetische Flüsse) linear unabhängig sind, lässt sich daraus immer die fehlende dritte Größe berechnen. Dafür kann eine Transformation eingeführt werden, welche das Dreiphasensystem in ein komplexes Zweiphasensystem mit nur zwei linear unabhängigen Größen überführt. Die realen drei Wicklungen werden damit durch zwei Wicklungen beschrieben, die senkrecht zueinander angeordnet sind. Die Transformationsvorschrift wird in der Literatur als Raumzeigertransformation bezeichnet [191], [208].

\section{Transformation von einem Drei- in ein Zweiphasensystem}

Aus der Addition der drei Stromkomponenten $i_{a}, i_{b}, i_{c}$ entsprechend ihrer Richtung in der komplexen Ebene resultiert der komplexe Statorstromraumzeiger $\mathbf{i}_{s}$ im statorfesten $\alpha$ - $\beta$-Koordinatensystem. Dabei wird die $\beta$-Achse als imaginäre Achse aufgefasst (vgl. Abbildung 5.9). Die $\alpha$-Achse wird in Richtung der $a$-Achse gelegt bzw. definiert, was die Beschreibung zusätzlich vereinfacht. Der komplexe Statorraumzeiger $\mathbf{i}_{s}$ berechnet sich dann gemäß Gl. (5.9) [181].
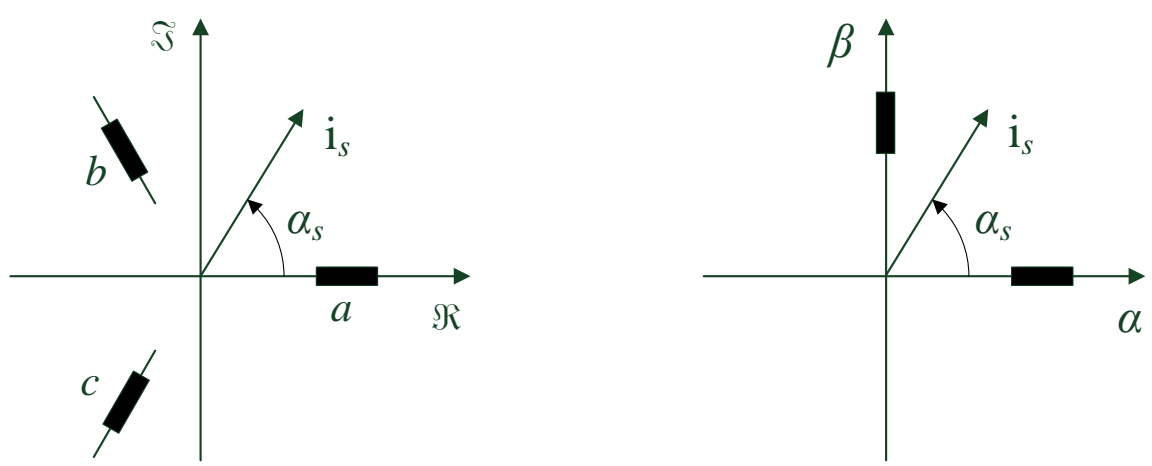

Abbildung 5.9.: Übergang vom Dreiphasensystem in das $\alpha, \beta$-Koordinatensystem [181]. 


$$
\begin{aligned}
\mathbf{i}_{s} & =i_{a}+\mathbf{a} i_{b}+\mathbf{a}^{2} i_{c} . \\
\mathbf{a} & =e^{j 120^{\circ}}=-\frac{1}{2}+j \frac{\sqrt{3}}{2} . \\
\mathbf{a}^{2} & =e^{j 240^{\circ}}=-\frac{1}{2}-j \frac{\sqrt{3}}{2} .
\end{aligned}
$$

Die komplexe Konstante a wird als Drehzeiger bezeichnet. Sie rechnet die Ströme im 3-Phasensystem entsprechend dem geometrischen Versatz der Spulen von $120^{\circ} \mathrm{bzw}$. $240^{\circ}$ in das 2-Phasensystem um. Entsprechendes gilt im Dreiphasensystem für die Spannung, den magnetischen Fluß und die Flußdichte. Multipliziert man Gl. (5.9) aus und trennt in Real- und Imaginärteil erhält man die Raumzeigerkomponenten $i_{\alpha}$ und $i_{\beta}$ des komplexen Zeigers $\mathbf{i}_{s}$. In Matrizenschreibweise folgt daraus Gl. (5.12) [181].

$$
\left[\begin{array}{c}
i_{\alpha} \\
i_{\beta}
\end{array}\right]=\frac{2}{3}\left[\begin{array}{ccc}
1 & -\frac{1}{2} & -\frac{1}{2} \\
0 & \frac{\sqrt{3}}{2} & -\frac{\sqrt{3}}{2}
\end{array}\right]\left[\begin{array}{c}
i_{a} \\
i_{b} \\
i_{c}
\end{array}\right]
$$

Die Matrizendarstellung in Gl. (5.12) beschreibt die räumliche Abhängigkeit des Raumzeigers $\mathbf{i}_{s}$ von den zeitlich veränderten Magnetfeldern in den Spulen $i_{a}, i_{b}, i_{c}$. Wenn durch die drei Spulen ein Wechselstrom fließt mit gleicher Amplitude, gleicher Frequenz und Phasenlage, d.h. $i_{a}(t)=i_{b}(t)=i_{c}(t)$, heben sich die $\alpha$-Komponenten und die $\beta$-Komponenten laut Gleichungssystem auf, d.h. die entstehenden Magnetfelder bzw. Durchflutungen neutralisieren sich gegenseitig. Der Sachverhalt ist durch die Symmetrie der drei angeordneten Statorspulen begründet.

In der Literatur ist es üblich, die Amplitude des Raumzeigers den Amplituden der Phasengrößen anzupassen. Als Faktor wird deswegen ein Skalierungsfaktor mit dem Wert 2/3 in Gl. (5.12) eingeführt. Der Raumzeiger $\mathbf{i}_{s}$ in Gl. (5.12) hat somit die gleiche Amplitude und die gleiche Frequenz wie die Phasengrößen und stimmt mit der Phasenlage von Phase $a$ überein. Die Gl. (5.12) vereinfacht sich, wenn entsprechend der Summe-Null-Bedingung $i_{a}+i_{b}+i_{c}=0$ der Strom $i_{c}$ durch: $i_{c}=-i_{a}-i_{b}$ ausgedrückt wird. Die Matrizenschreibweise der Gl. (5.12) wird dann gemäß Gl. (5.13) beschrieben. 


$$
\left[\begin{array}{c}
i_{\alpha} \\
i_{\beta}
\end{array}\right]=\left[\begin{array}{cc}
1 & 0 \\
\frac{1}{\sqrt{3}} & \frac{2}{\sqrt{3}}
\end{array}\right]\left[\begin{array}{c}
i_{a} \\
i_{b}
\end{array}\right] .
$$

In Gl. (5.13) wird deutlich, dass unter Berücksichtung der Summe-Null-Bedingung für die Bestimmung der $\alpha$ - und $\beta$-Komponente nur zwei Phasenströme benötigt werden. Für die praktische Umsetzung der Motorstrommessung sind deshalb nur zwei Stromwandler notwendig. Bei der Umsetzung der Motorregelung in Abschnitt 5.3.5 werden für die Strommessung des Motors entsprechend zwei der drei Phasenströme ( $u$ - und $w$-Wicklung) abgegriffen (vgl. Abbildung 5.33). Für die Auswertung der Phasenströme in der $u$ - und $w$-Wicklung ergibt sich ausgehend von Glg. 5.12 und unter Berücksichtigung der Summe-Null-Bedingung mit: $i_{b}=-i_{a}-i_{c}$ die Gl. (5.14).

$$
\left[\begin{array}{c}
i_{\alpha} \\
i_{\beta}
\end{array}\right]=\left[\begin{array}{cc}
1 & 0 \\
-\frac{1}{\sqrt{3}} & -\frac{2}{\sqrt{3}}
\end{array}\right]\left[\begin{array}{l}
i_{a} \\
i_{c}
\end{array}\right] .
$$

Die Transformation vom $a, b, c$-Koordinatensystem in das $\alpha, \beta$-Koordinatensystem wird in der Literatur als Clarke-Transformation ${ }^{3}$ bezeichnet. Die invertierte Matrix ergibt sich gemäß Gl. (5.15) [181].

$$
\left[\begin{array}{c}
i_{a} \\
i_{b} \\
i_{c}
\end{array}\right]=\left[\begin{array}{cc}
1 & 0 \\
-\frac{1}{2} & \frac{\sqrt{3}}{2} \\
-\frac{1}{2} & -\frac{\sqrt{3}}{2}
\end{array}\right]\left[\begin{array}{l}
i_{\alpha} \\
i_{\beta}
\end{array}\right] .
$$

\section{Transformation in das $d, q$-Koordinatensystem}

Eine weitere wichtige Transformationsvorschrift ist die sogenannte $d, q$-Transformation, auch als Park-Transformation ${ }^{4}$ bezeichnet. Sie beschreibt ursprünglich die Überführung dreiphasiger Größen mit den Achsen $a, b, c$ in ein umlaufendes zweiachsiges Koordinatensystem.

Die Betrachtung der beschriebenen Größen bezieht sich jetzt nicht mehr auf den „feststehenden" Stator, sondern auf den Rotationsvorgang selbst. Im $d, q$-System dreht sich der "Betrachter" mit dem Rotorsystem mit, was einer Überführung von Wechselgrößen in Gleichgrößen entspricht. Regelungstechnisch lassen sich die Gleichgrößen, z.B. bei Anwendung der feldorientierten Regelung, einfacher berechnen. Unter

\footnotetext{
${ }^{3}$ benannt nach Edith Clarke

${ }^{4}$ nach Robert H. Park, erstmals 1929 beschrieben
} 
Verwendung der im letzten Abschnitt beschriebenen $\alpha, \beta$-Koordinatenwandlung reduziert sich die Koordinatenumrechnung auf die Überführung eines stationären Zweiphasensystems in ein umlaufendes Zweiphasensystem (Gleichgrößensystem). Abbildung 5.10 beschreibt den Übergang des Koordinatensystems.

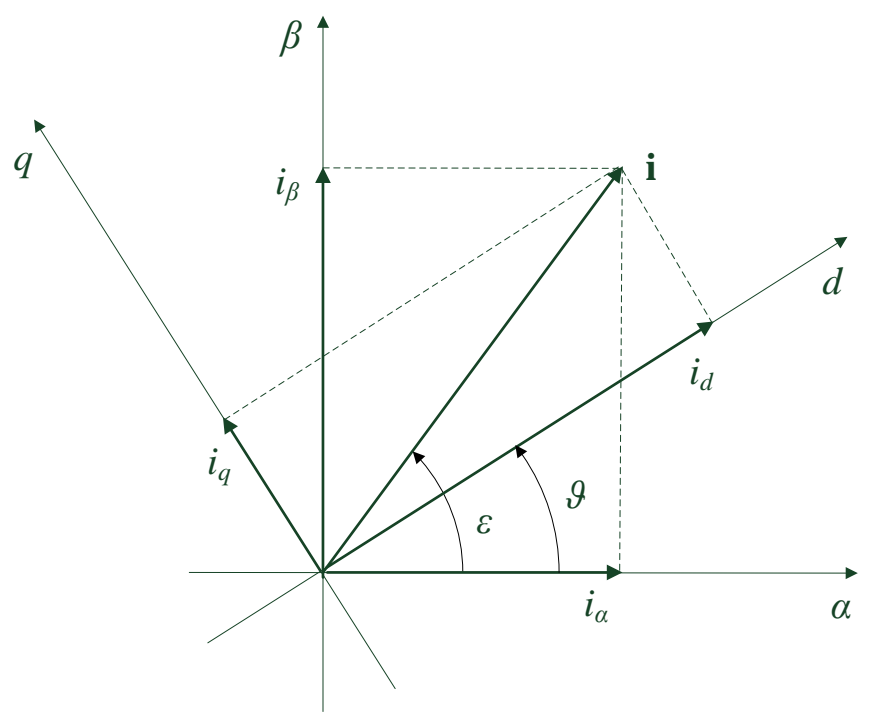

Abbildung 5.10.: Zusammenhang zwischen $\alpha, \beta$ - und $d, q$-Koordinatensystem [181].

In Abbildung 5.10 gilt für die einzelnen Komponenten $i_{\alpha}, i_{\beta}, i_{d}, i_{q}$ der Zusammenhang zum umlaufenden Zeiger i gemäß Gl. (5.16) und Gl. (5.17) [181].

$$
\begin{aligned}
& i_{\alpha}+j i_{\beta}=\mathbf{i} e^{j \epsilon} . \\
& i_{d}+j i_{q}=\mathbf{i} e^{j(\epsilon-\vartheta)} .
\end{aligned}
$$

Setzt man Gl. (5.16) in Gl. (5.17) ein, folgt daraus Gl. (5.18).

$$
i_{d}+j i_{q}=\left(i_{\alpha}+j i_{\beta}\right) e^{-j \vartheta}
$$

Nach Ausmultiplizieren und Sortieren der Gl. (5.18) folgt durch Koeffizientenvergleich der reellen und komplexen Komponenten Gl. (5.19) und Gl. (5.20). 


$$
\begin{aligned}
& i_{d}=i_{\alpha} \cos \vartheta+i_{\beta} \sin \vartheta \\
& i_{q}=-i_{\alpha} \sin \vartheta+i_{\beta} \cos \vartheta .
\end{aligned}
$$

In Matrixschreibweise:

$$
\left[\begin{array}{c}
i_{d} \\
i_{q}
\end{array}\right]=\left[\begin{array}{cc}
\cos \vartheta & \sin \vartheta \\
-\sin \vartheta & \cos \vartheta
\end{array}\right]\left[\begin{array}{c}
i_{\alpha} \\
i_{\beta}
\end{array}\right] .
$$

Die inverse Matrix lautet dann:

$$
\left[\begin{array}{c}
i_{\alpha} \\
i_{\beta}
\end{array}\right]=\left[\begin{array}{cc}
\cos \vartheta & -\sin \vartheta \\
\sin \vartheta & \cos \vartheta
\end{array}\right]\left[\begin{array}{c}
i_{d} \\
i_{q}
\end{array}\right]
$$

\subsubsection{Feldorientierte Regelung}

Neben dem Verfahren der U/F-Regelung und der direkten Drehmomentregelung (DTC, engl.: Direct Torque Control) bildet vor allem die feldorientierte Regelung oder Vector Control) ein Standard-Regelverfahren für dynamische und hochdynamische Drehstromantriebe [157], [181], [190]. Das Blockschaltbild der FOC für die Drehzahl- und Positionsregelung einer PMSM ist in Abbildung 5.11 dargestellt.

Ziel des Regelungsverfahrens ist die entkoppelte Regelung von Fluss und Drehmoment. Durch die Vorgehensweise erreicht man ein vergleichbares Verhalten wie bei einer Gleichstromnebenschlußmaschine [63]. Der drehmomentbildende Strom $i_{q}$ entspricht dabei dem Ankerstrom, der feldbildende Strom $i_{d}$ dem Erregerstrom der Gleichstrommaschine. Das erzeugte innere Drehmoment des Motors ist bei dem Regelverfahren entsprechend der Gleichstrommaschine direkt proportional zum Strom $i_{q}$. Die flussbildende Stromkomponente $i_{d}$ leistet keinen Beitrag zur Drehmomentbildung und wird üblicherweise bei der PMSM zu Null geregelt, da ja der magnetische Fluss permanent durch die Dauermagnete gegeben ist. Durch die Veränderung von $i_{d}$ besteht allerdings auch die Möglichkeit der Feldschwächung.

Im Unterschied zur Gleichstrommaschine sind die Komponenten der Raumzeiger für Strom und Spannung $i_{q}, i_{d}, u_{q}$ und $u_{d}$ allerdings nicht raumfest sondern drehen sich mit dem Rotor mit. Die gemessenen Ströme in der Statorwicklung müssen deshalb mit den im Abschnitt 5.3.2 beschriebenen Transformationsgleichungen, Gl. (5.13) und Gl. 


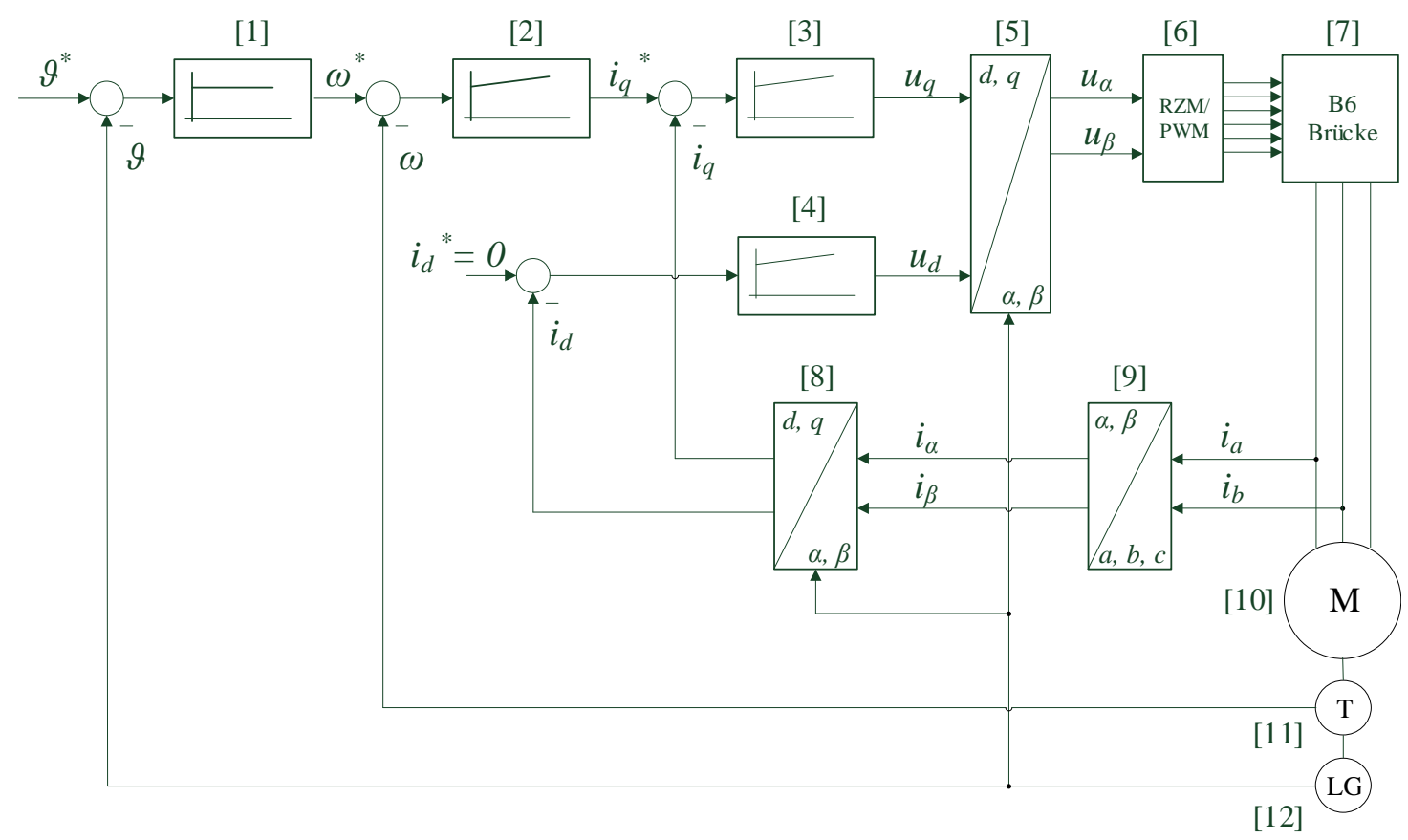

Abbildung 5.11.: Feldorientierte Regelung der PMSM [181].

[1] P-Lageregler, [2] PI- Drehzahlregler, [3] PI-Stromregler q-Achse, [4] PI-Stromregler $d$-Achse, [5] inverse Park-Transformation, [6] Raumzeigermodulation (RZM) mit Pulsweitenmodulation (PWM), [7] Treiberbaustein zur Ansteuerung der Leistungshalbleiter, [8] Park-Transformation, [9] Clarke-Transformation, [10] Motor (PMSM), [11] Tacho zur Drehzahlerfassung, [12] Lagegeber zur Rotorwinkelerfassung.

(5.21) (Clarke- und Parktransformation) in das rotierende $d, q$-Koordinatensystem umgerechnet werden.

In Gl. (5.23) sind die zwei Umrechnungsschritte beispielhaft zusammengefasst [181]. Dank schneller Prozessoren und schneller Stromwandler ist die Umrechnung „online" mit den heute verfügbaren elektronischen Bauteilen effektiv und kostengünstig realisierbar.

$$
\left[\begin{array}{c}
i_{d} \\
i_{q}
\end{array}\right]=\frac{2}{3}\left[\begin{array}{ccc}
\cos \vartheta & \cos \left(\vartheta-\frac{2 \pi}{3}\right) & \cos \left(\vartheta+\frac{2 \pi}{3}\right) \\
-\sin \vartheta & -\sin \left(\vartheta-\frac{2 \pi}{3}\right) & -\sin \left(\vartheta+\frac{2 \pi}{3}\right)
\end{array}\right]\left[\begin{array}{c}
i_{a} \\
i_{b} \\
i_{c}
\end{array}\right]
$$


Der Aufbau der Regelung gemäß Abbildung 5.11 entspricht der einer bewährten Kaskadenregelung. Die einzelnen Regelkreise (Lage-, Drehzahl- und Stromregelkreis) ergeben eine übersichtliche Struktur und sind einzeln einstellbar. Der äußerste Kreis bildet die Lageregelung, die durch einen einfachen P-Regler realisiert ist. Das Ausgangssignal des Drehzahlreglers liefert den Sollwert für den Stromregler der drehmomentbestimmenden Stromkomponente $i_{q}$. Die beiden PI-Stromregler bilden den innersten Kreis der Kaskadenregelung.

Als Stellgrößen am Ausgang der Stromregler erhält man die Komponenten der Motorspannung $u_{d}$ und $u_{q}$. Der rotierende Spannungsraumzeiger im $d, q$-Koordinatensystem wird dann zuerst mittels der inversen Parktransformation, Gl. (5.22), in das $\alpha, \beta$ Koordinatensystem überführt. Die Werte der Komponenten $u_{\alpha}$ und $u_{\beta}$ bilden die Stellwerte für die nachgeschaltete Raumzeigermodulation (RZM) und Pulsweitenmodulation (PWM). Aus den berechneten Werten der Ergebnisse werden die Steuersignale für den Wechselrichter gebildet. Abbildung 5.12 gibt einen Überblick über die in der Regelung verwendeten Transformationen [181].

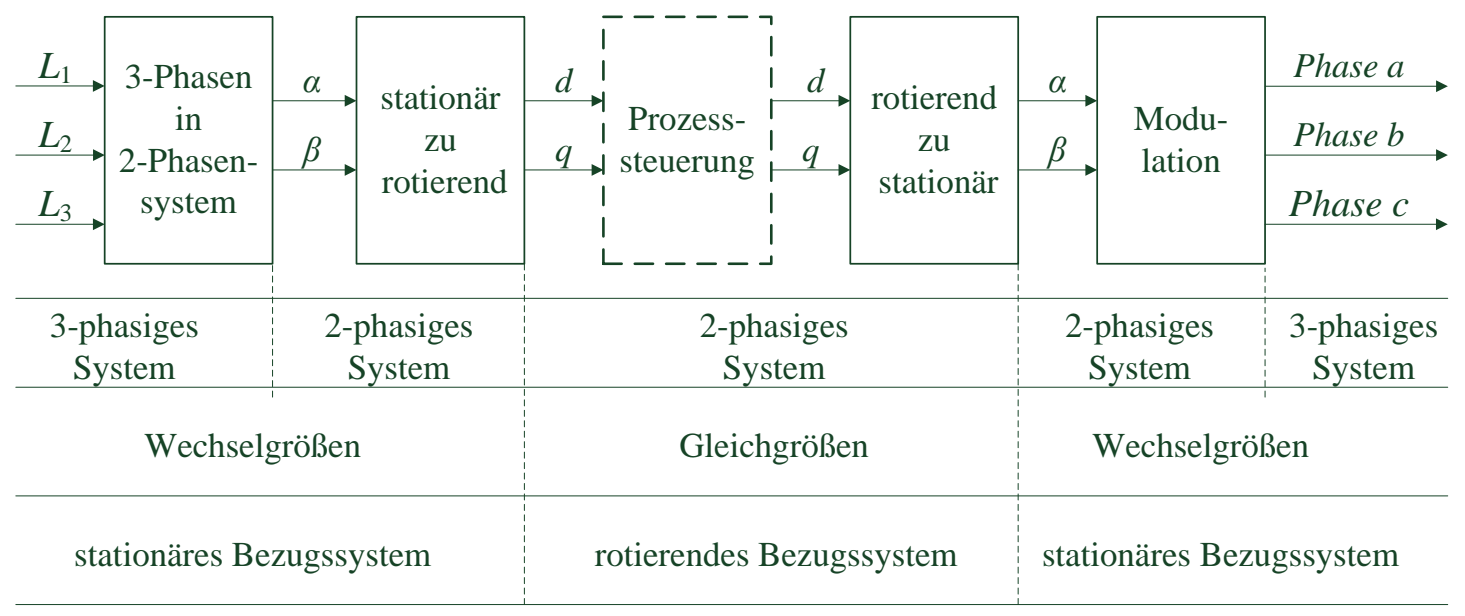

Abbildung 5.12.: Übersicht Transformationen [181].

\section{Stromreglung mit Entkopplung}

Im Modell der PMSM im Rotorkoordinatensystem (vgl. Abbildung 6.5, Gln. (6.48) und (6.49)) wird deutlich, dass die beiden Achsen des Motormodells fest miteinander gekoppelt sind und sich dadurch gegenseitig beeinflussen. Der Strom $i_{q}$ beeinflusst $u_{d}$, aber auch umgekehrt beeinflusst $i_{d}$ die Spannungskomponente $u_{q}$. Eine weitere Störgröße bildet die drehzahlabhängige Polradspannung $u_{p}=\omega \psi_{P M}$. Eine Entflechtung kann durch eine Kreuzentkopplung erreicht werden, indem die Werte aus den Messgrößen bestimmt werden und zur Entkopplung den Stellgrößen der Stromregler 
überlagert werden. Man kann die gegenseitigen Beeinflussungen allerdings auch als generelle Störgrößen auffassen, die dann über die PI-Regler ausgeregelt werden.

Die Gln. (5.24) und (5.25) beschreiben die Spannungsgleichungen der PMSM in Rotorkoordinaten [181] (vgl. Abschnitt 6.2.2). Ist es möglich, Fluss und Drehmoment im Stromregler unabhängig voneinander zu regeln, ergibt sich regelungstechnisch ein einfacheres Regelungsverfahren. Ziel des Stromreglers sollte es deshalb sein, eine ideale Entkopplung der feldbildenden Stromkomponente $i_{d}$ und der momentenbildenden Stromkomponente $i_{q}$ zu erreichen [144], [186]. Eine Entkopplung ist möglich, wenn das Entkopplungsnetzwerk dabei das inverse Übertragungsverhalten zur PMSM aufweist [190]. Für die Entkopplung können die Gln. (5.24) und (5.25) in lineare und verkoppelte Komponenten aufgeteilt werden [181].

$$
\begin{aligned}
& u_{d}=R_{s} i_{d}+L_{d} \frac{d}{d t} i_{d}-L_{q} \omega i_{q}=u_{d(\text { lin })}-u_{d(\text { kopp })} . \\
& u_{q}=R_{s} i_{q}+L_{q} \frac{d}{d t} i_{q}+L_{d} \omega i_{d}+\psi_{P M} \omega=u_{q(\text { lin })}+u_{q(\text { kopp })} .
\end{aligned}
$$

Für die linearen Spannungskomponenten aus den Gln. (5.24) und (5.25) am Ausgang des Stromreglers gelten entsprechend die Gln. (5.26) und (5.27).

$$
\begin{aligned}
& u_{d(\text { lin })}=R_{s} i_{d}+\frac{d}{d t} \psi_{d} . \\
& u_{q(\text { lin })}=R_{s} i_{q}+\frac{d}{d t} \psi_{q} .
\end{aligned}
$$

Für die verkoppelten Spannungskomponenten aus der Gl. (5.24) und Gl. (5.25) am Ausgang des Entkopplungsnetzwerkes gelten entsprechend Gl. (5.28) und Gl. $(5.29)$.

$$
\begin{aligned}
& u_{d(\text { kopp })}=L_{q} \omega i_{q} . \\
& u_{q(\text { kopp })}=\omega\left(L_{d} i_{d}+\psi_{P M}\right) .
\end{aligned}
$$

Abbildung 5.13 zeigt die Zweigrößenregelung des Stromreglers, d.h. die Regelung der Gleichgrößen $i_{d}$ und $i_{q}$ jetzt ergänzt mit dem entsprechenden Entkopplungsnetzwerk. Durch die Entkopplung wird das nichtlineare Motormodell quasi in ein lineares Modell überführt. 


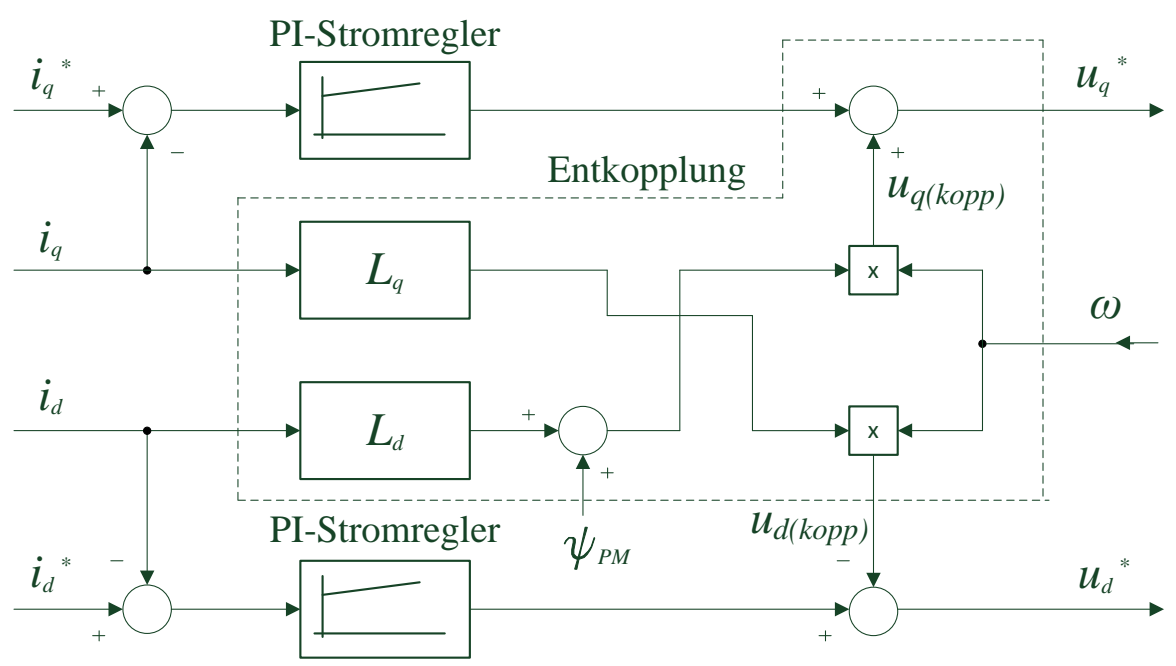

Abbildung 5.13.: Blockschaltbild der Stromregelung mit Entkopplung [181].

\section{Stromregelkreis der Motorregelung}

Der Stromregelkreis bestimmt als innerster Kreis maßgeblich die Dynamik des Antriebstranges. Durch die Anwendung der FOC ist es möglich, die beiden Stromkomponenten in der $d$ - und $q$-Ebene separat zu regeln, d.h. die Stromregelung erfolgt im rotororientierten Koordinatensystem. Da im Rotorkoordinatensystem wie erläutert nur Gleichgrößen auftreten, ist die Verwendung zweier einfacher PI-Regler möglich.

Für eine permanenterregte Drehfeldmaschine ohne Reluktanzeinflüsse $\left(L_{d} \approx L_{q}\right)$ ergeben sich für die Reglersynthese im $d$ - und $q$-Zweig keine markanten Unterschiede. Weiter gilt die Proportionalitäts-Betrachtung zwischen Strom $i_{q}$ und Motormoment. In der kaskadenförmigen Struktur der Drehzahlregelung bildet das Ausgangssignal des Drehzahlreglers das Eingangssignal des $i_{q}$-Stromreglers. Der $i_{q}$-Regler stellt den momentenbildenden Strom zur Verfügung, der Sollwert für den Regler im $d$-Zweig der Stromregelung wird mit $i_{d}{ }^{*}=0$ vorgegeben. Ziel der Stromregelung ist es, die Abweichungen vom Drehzahlsollwert möglichst schnell auszuregeln. Abbildung 5.14 zeigt den vereinfachten Aufbau des unterlagerten Stromregelkreises im $q$-Zweig.

Im Vorwärtszweig sind die Übertragungsfunktionen des PI-Reglers, der Stelleinrichtung und des Motors eingezeichnet. Die Stelleinrichtung lässt sich näherungsweise durch ein $\mathrm{PT}_{1}$-Glied mit der Zeitkonstante $T_{S t}$ beschreiben. Verzögerungszeiten entstehen generell in der Stelleinrichtung bzw. im Umrichter u.a. durch die Pulsweitenmodulation und der Bearbeitungszeiten der Prozessoren, wie sie heute üblicherweise bei der Umsetzung in eine zeitdiskrete Reglerrealisierung auftreten. Da die Zeitkonstanten aber deutlich kleiner sind als die im System auftretenden elektrischen Zeitkonstanten der Maschine ist eine quasi-kontinuierliche Betrachtungsweise 


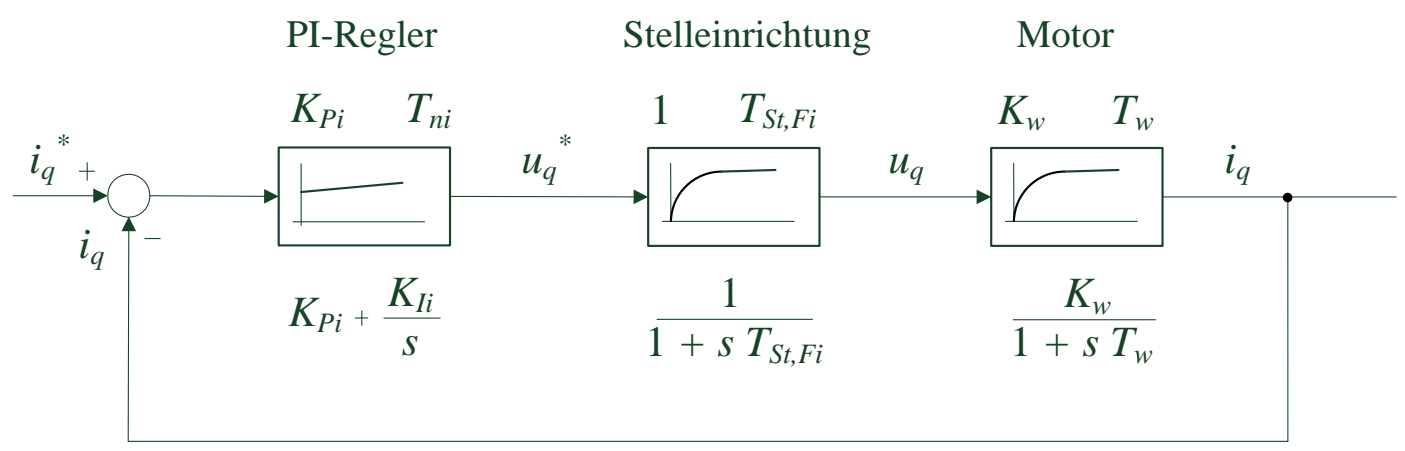

Abbildung 5.14.: Vereinfachter Stromregelkreis mit Ersatzzeitkonstante [181].

im s-Bereich möglich. Alternativ besteht die Möglichkeit, eine summarische Totzeit einzuführen [181].

Die normalerweise im Rückführzweig in Abbildung 5.14 befindliche Messeinrichtung für die Strommessung bzw. der Stromfilter wurde bereits in den Vorwärtszweig umgerechnet. Der Stromfilter unterdrückt das Rauschen im Istwert-Signal der Strommessung bzw. eliminiert Oberschwingungen, kann aber das Signal auch merklich verzögern. Der Stromfilter wird in der Regel als $\mathrm{PT}_{1}$-Glied bzw. als Tiefpass dargestellt. Die kleine Zeitkonstante des Stromfilters $T_{F i}$ und die Zeitkonstante des Stellgliedes $T_{S t}$ wurden in Abbildung 5.14 durch die Ersatzzeitkonstante $T_{S t, F i}$ zusammengefasst, Gl. (5.30).

$$
T_{S t, F i}=T_{S t}+T_{F i}
$$

Das letzte Übertragungsglied im Vorwärtszweig ist die Regelstrecke bzw. die Motorwicklung. Die Regelstrecke kann gleichfalls näherungsweise als Verzögerungsglied 1. Ordnung bzw. als $\mathrm{PT}_{1}$-Glied modelliert werden. Die Zeitkonstante $T_{w}=L_{s} / R_{s}$ berechnet sich aus dem Statorwiderstand $R_{s}$ und der Statorinduktivität $L_{s}$. Sie bildet die größte Zeitkonstante im Stromregelkreis.

Die drehzahlabhängige induzierte Gegenspannung $U_{i}$ wurde in Abbildung 5.14 nicht berücksichtigt. Sie kann als vor der Strecke eingreifende Störung betrachtet werden. Im Vergleich zur Dynamik der Stromänderung ändert sich die Drehzahl des Motors in der Regel nur sehr wenig, ein anderes Verhalten besteht bei hochdynamischen Anwendungen und gegebenen geringen Massenträgheitsverhältnissen. Der in Abbildung 5.14 dargestellte PI-Regler mit den in Reihe geschalteten zwei $\mathrm{PT}_{1}$-Gliedern führt mit $K_{I i}=K_{P i} / T_{n i}$ zur Gl. (5.31) des offenen Regelkreises.

$$
G_{o i}(s)=K_{P i} \frac{1+s T_{n i}}{s T_{n i}} \frac{1}{1+s T_{S t, F i}} \frac{K_{w}}{1+s T_{w}}
$$


Die Strecke selbst besitzt kein I-Anteil. Entsprechend der Auslegung nach dem Betragsoptimum wird die größte Zeitkonstante des offenen Kreises mit der Nachstellzeit $T_{n i}$ des Reglers kompensiert. Für die Parameter des PI-Reglers ergeben sich folgende Einstellwerte [190], Gln. (5.32),(5.33).

$$
\begin{aligned}
T_{n i} & =T_{w} . \\
K_{P i} & =\frac{T_{w}}{2 K_{w} T_{S t, F i}} .
\end{aligned}
$$

Für den Wert der Reglerverstärkung $K_{P i}$ und der Nachstellzeit $T_{n i}$ wird für den geschlossenen Regelkreis ein Wert für die Dämpfung von $d=1 / \sqrt{2} \approx 0,7$ erreicht [181], [186]. Da im Allgemeinen nicht alle Komponenten und Einflüsse der Regelstrecke bekannt sind, ist die genaue Festlegung der Regelparameter selbstverständlich nur annähernd möglich.

\section{Drehzahlregelkreis der Motorregelung}

Abbildung 5.15 zeigt den Aufbau des Drehzahlregelkreises. Die Regelstrecke enthält den unterlagerten Wirkstromregelkreis mit Stellvorrichtung und Motor und als integralen Bestandteil den angekoppelten mechanischen Aufbau der Arbeitsmaschine. $K_{T}$ steht für die Drehmomentkonstante, $J$ für die Massenträgheit des Antriebsstranges. Das Lastdrehmoment ist als eine von außen eingreifende Störgröße in der Abbildung 5.15 nicht eingezeichnet.

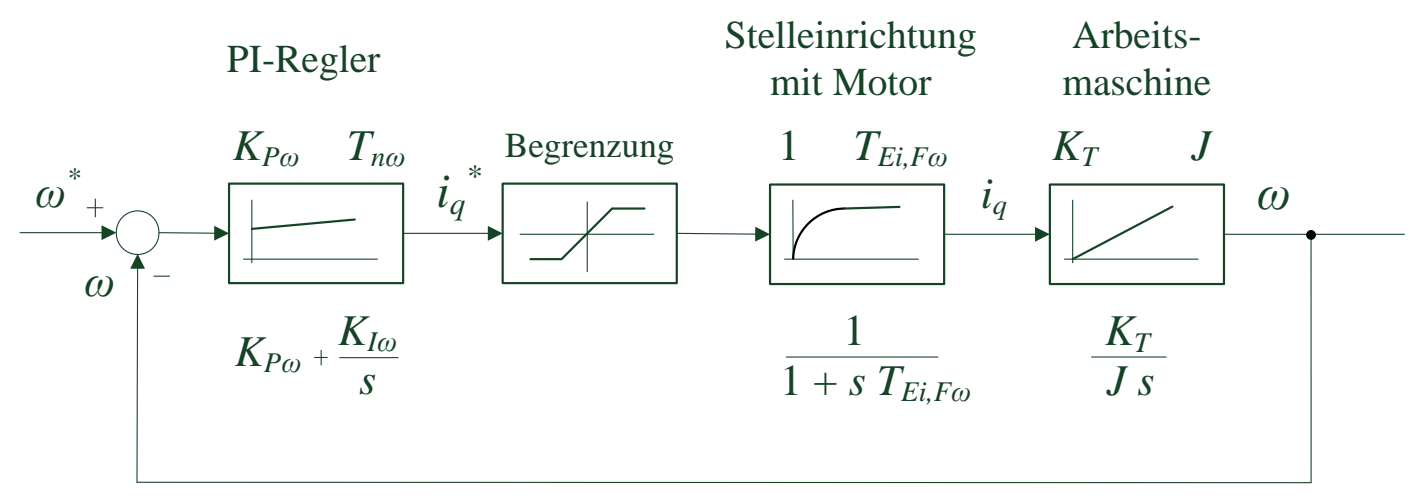

Abbildung 5.15.: Vereinfachter Drehzahlregelkreis mit Ersatzzeitkonstante [181].

Die Aufgabe des Drehzahlreglers ist es, durch die Vorgabe des Drehmomentsollwertes die Strecke zu regeln. Ziel des unterlagerten Stromregelkreises ist es, durch die 
schnelle Einprägung des Stromes in der Motorwicklung das geforderte Motordrehmoment bereitzustellen. Der geschlossene Stromregelkreis kann näherungsweise durch ein $\mathrm{PT}_{1}$-Glied mit der Verzögerungszeitkonstante bzw. Ersatzzeitkonstante $T_{E i}$ dargestellt werden. Durch die Ordnungsreduktion des Stromregelkreises vereinfacht sich der Reglerentwurf des Drehzahlregelkreises. Die Übertragungsfunktion der Messvorrichtung zur Drehzahlbestimmung im Rückwärtszweig des Drehzahlregelkreises ( $\mathrm{PT}_{1}$-Verhalten) wurde bereits in Abbildung 5.15 in den Vorwärtszweig umgerechnet.

Bei der Verwendung moderner Mikroprozessoren wird die Geschwindigkeit zwischen zwei Abtastpunkten ermittelt. Durch die Differenziation kann immer nur die mittlere Geschwindigkeit innerhalb des Abtastintervalls bestimmt werden. Die begrenzte Auflösung des Gebersystems und der zeitliche Fehler bei der Bestimmung des exakten Abtastintervalls führen u.a. zu Rauschen und Ungenauigkeiten im Drehzahlsignal. Die Messvorrichtung bzw. der Tiefpassfilter reduzieren das Rauschsignal, beeinflussen aber unter Umständen die Dynamik der Regelung negativ. Die beiden kleinen Zeitkonstanten $T_{F \omega}$ des Tiefpassfilters des Drehzahlgebers und die Ersatzzeitkonstante des Stromregelkreises $T_{E i}$ werden aus Vereinfachungsgründen durch die Ersatzzeitkonstante $T_{E i, F \omega}$ zusammengefasst, die Zeitkonstanten sind durch den Regler nicht kompensierbar, Gl. (5.34).

$$
T_{E i, F \omega}=T_{E i}+T_{F \omega} .
$$

Der Drehzahlregler ist Bestandteil der Kaskadenstruktur der Regelung und wird typischerweise als PI-Regler ausgelegt. Dadurch ist gewährleistet, dass das Lastmoment keine bleibenden Regelabweichungen verursacht. Der Entwurf der Regelung erfolgt in der Regel nach dem symmetrischen Optimum [63]. Für die Übertragungsfunktion des offenen Kreises ergibt sich gemäß Abbildung 5.15, mit $K_{I \omega}=K_{P \omega} / T_{n \omega}$, Gl. (5.35).

$$
G_{o \omega}(s)=K_{P \omega} \frac{1+s T_{n \omega}}{s T_{n \omega}} \frac{1}{1+s T_{E i, F \omega}} \frac{K_{T}}{s J} .
$$

Für die Parameter des PI-Reglers ergeben sich bei Anwendung nach den Vorgaben des symmetrischen Optimum die Einstellwerte für die Nachstellzeit $T_{n \omega}$, Gl. (5.36) und für die Reglerverstärkung $K_{P \omega}$, Gl. (5.37), [190].

$$
\begin{aligned}
T_{n \omega} & =4 T_{E i, F \omega} . \\
K_{P \omega} & =\frac{J}{2 K_{T} T_{E i, F \omega}} .
\end{aligned}
$$


Die ermittelten Einstellwerte in den Gln. (5.36), (5.37) stellen wie bei den Vorgaben des Stromreglers Näherungswerte dar, die für die konkrete Applikation überprüft werden müssen. Die Reglerverstärkung am PI-Regler kann bekanntermaßen nur bis zur Stabilitätsgrenze der Regelung angehoben werden. Bei Überschreiten der Grenze beginnt der Drehzahlregelkreis zu schwingen. Wesentlich sind dabei die bereits beschriebenen Faktoren wie die Dynamik des unterlagerten Stromreglers, die Rechenzeit des verwendeten Prozessors, die Abtastfrequenz des Drehzahlreglers und die schnelle und korrekte Erfassung der Drehzahl. Die Aufgabe des Integrators des Drehzahlreglers ist, eine mögliche bleibende Regeldifferenz auszuregeln, er hat keinen wesentlichen Einfluss auf die Dynamik des Drehzahlregelkreises.

\section{Drehzahlregelkreis mit Beschleunigungs-Vorsteuerung}

Zur Verbesserung des Regelkreis-Führungsverhaltens wird in der Praxis oft eine Beschleunigungs-Vorsteuerung in den Drehzahlregelkreis integriert [63], [127], [190]. Abbildung 5.16 zeigt die Erweiterung der Struktur parallel zum klassischen PIRegler.

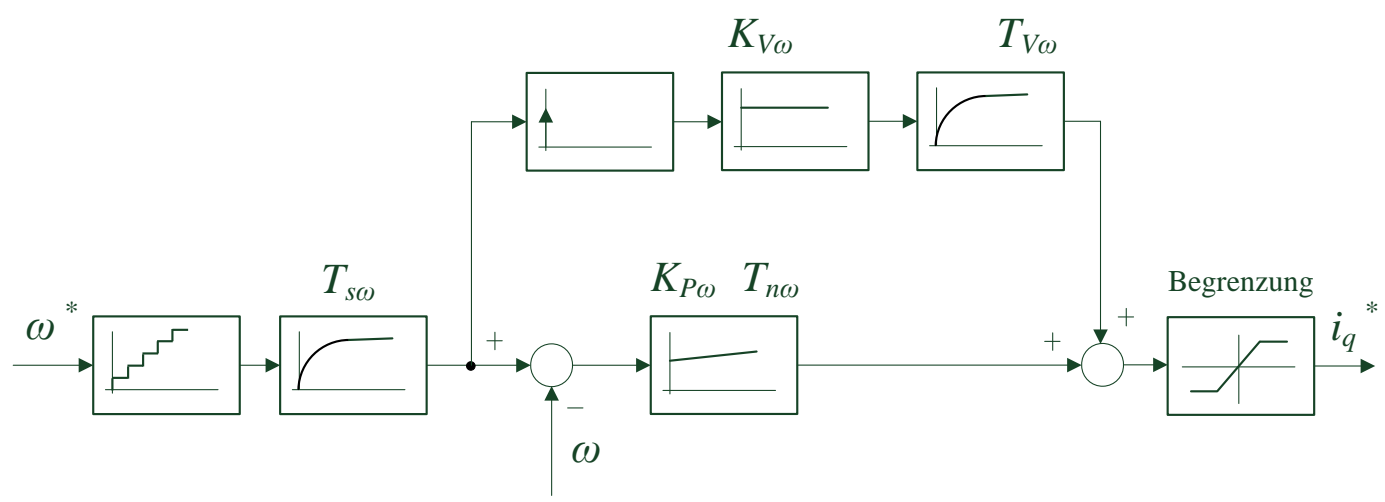

Abbildung 5.16.: Drehzahlregelkreis mit Beschleunigungs-Vorsteuerung [127].

Bei großen Änderungen der Führungsgröße wird zusätzlich ein Impuls über das Differenzierglied auf den Stromregler geschaltet, d.h. der Antrieb kann bereits ein Drehmoment an der Motorwelle aufbauen, bevor eine Regeldifferenz durch den Drehzahlregler erkannt wird. Die Zeitkonstante des Regelkreises wird nicht beeinflusst - aber auch nicht die Dynamik der Störgrößenaufschaltung. Ggf. müssen noch zusätzliche Filter integriert werden, um das Rauschen des Drehzahlsignals zu unterdrücken bzw. um den Drehmoment-Ripple klein zu halten, allerdings haben die Maßnahmen wieder eine verzögerte Wirkung auf die Dynamik der Regelung. 


\section{Regler Windup}

Für die überlagerte Drehzahlregelung des Motors wird, wie bereits in Abbildung 5.11 dargestellt, ein PI-Regler verwendet. Jeder reale Regler unterliegt dabei einer Stellgrößenbegrenzung. Aufgeschaltete große Führungsgrößen oder Störgrößen können dazu führen, dass das Stellsignal die Aussteuergrenze erreicht - das gilt sowohl für eine Überschreitung als auch für eine Unterschreitung der Begrenzung.

Ist die Stellgrößenbegrenzung aktiv, kann es bei einem Regler mit I-Anteil zu Problemen führen. Durch die vorhandene Begrenzung der Stellgröße kann die Regelgröße in der Regel ihren Wert nur langsam ändern, dabei steht die Regeldifferenz weiterhin am Eingang des PI-Reglers an, was dazu führt, dass der I-Anteil des Reglers aufintegriert bzw. weiter ansteigt. Ein geschlossener Regelkreis ist quasi nicht mehr gegeben, das Regelsystem arbeitet im Open Loop.

Überschreitet die Regelgröße den vorgegebenen Sollwert, d.h. am Eingang des PIReglers wechselt das Vorzeichen, muss der I-Anteil des Reglers seinen angewachsenen integrierten Anteil zuerst wieder abbauen. Ein solches Verhalten wird als WindupEffekt bezeichnet [63], [190], [149]. Die Regelung neigt in dem Fall zu einem starken Überschwingverhalten, insgesamt zu einem schwach gedämpften Regelverhalten. Abbildung 5.17 zeigt das Blockschaltbild des PI-Reglers mit erweiterter Anti-Windup Struktur.

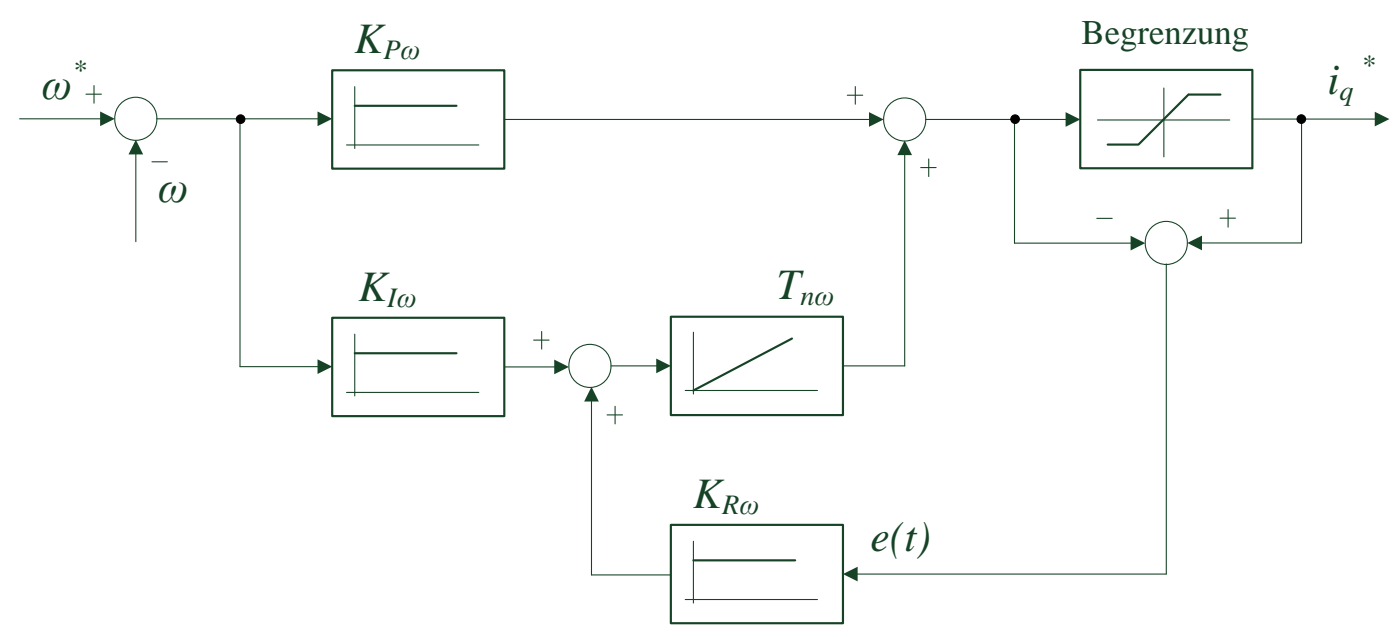

Abbildung 5.17.: PI-Regler mit Anti-Windup Struktur [190].

Das Differenzsignal $e(t)$ wird vor und nach der Begrenzung ausgewertet und auf den I-Anteil des PI-Reglers zurückgeführt. Mit dem Wert $K_{R \omega}$ kann die Verstärkung des zurückgeführten Signals eingestellt werden. Befindet sich das Sollwertsignal am 
Ausgang des PI-Reglers noch nicht im Aussteuerbereich, wird der I-Anteil des PIReglers nicht beeinflusst, d.h. $e(t)=0$. Wird die Aussteuergrenze z.B. überschritten, wird der I-Anteil im Regler reduziert.

Eine andere Möglichkeit, den Windup-Effekt einzugrenzen, besteht darin, das Eingangssignal des PI-Reglers (vor der $K_{I}$ Verstärkung) bei Erreichen der Stellgrößenbegrenzung auf den Wert Null zu setzen.

\subsubsection{Darstellung der Raumzeigermodulation}

Die Raumzeigermodulation (RZM) (engl.: Space Vector Modulation, SVM) bezeichnet ein Verfahren für die Ansteuerung von Drehstrommaschinen oder anderen rotierenden elektrischen Maschinen auf Basis der Pulsweitenmodulation [190]. Die Modulation ermöglicht die Nachbildung eines Mehrphasensystems auf elektronischem Wege.

Der Leistungsteil eines Frequenzumrichters oder Servoverstärkers zur Ansteuerung von Drehstrommotoren basiert in der Regel auf dem Prinzip des Spannungszwischenkreis-Verstärkers. Abbildung 5.18 zeigt den klassischen Aufbau der Schaltung.

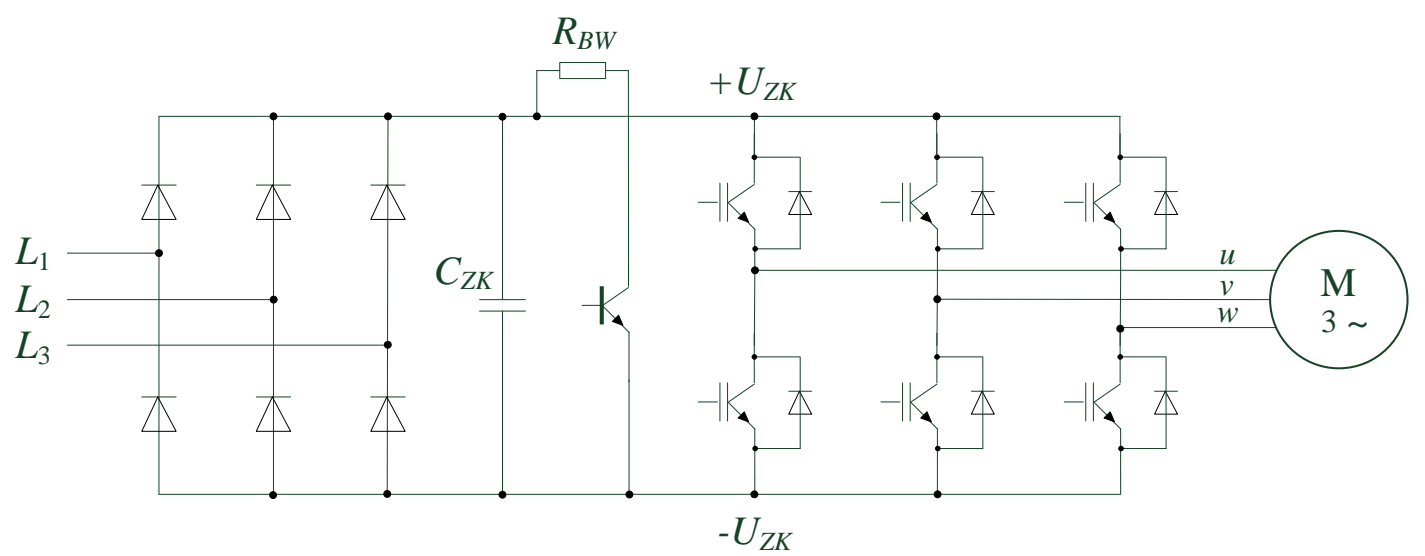

Abbildung 5.18.: Aufbau eines Spannungszwischenkreis-Umrichters [189].

Eine Brückenschaltung, hier im Bild eine B6-Diodenbrücke [31], richtet die dreiphasige sinusförmige (Netz)-Eingangswechselspannung im ersten Schritt in eine Gleichspannung um, die den Gleichspannungs-Zwischenkreis versorgt. Der nachgeschaltete Zwischenkreiskondensator $C_{Z K}$ hat neben der Glättungsfunktion der Zwischenkreisspannung vor allem die Aufgabe, die Energie kurzzeitig im Zwischenkreis zu puffern bzw. zu speichern. Im Fachjargon spricht man bei geringen Kapazitäten von einem „dünnen“ Zwischenkreis, bei hohen Kapazitäten von einem „dicken“ Zwischenkreis [196]. 
Somit dient der Zwischenkreis als Kopplungselement zwischen dem eingangsseitigen Gleichrichter und dem ausgangseitigen Wechselrichter. Weiterhin hat der Kondensator die Aufgabe, im generatorischen Betrieb des Motors die freiwerdende kinetische Energie in elektrische Energie zurückzuwandeln und zwischenzuspeichern. Steigt die Spannung im Zwischenkreis über einen kritischen Wert, wird die überschüssige Energie über den Bremswiderstand $R_{B W}$ in Wärme umgewandelt. Alternativ besteht die Möglichkeit, bei der Verwendung von mehreren Umrichtern die Zwischenkreise der Umrichter miteinander zu koppeln oder bei entsprechender Ausstattung der Eingangsbeschaltung die Möglichkeit der Netzrückspeisung.

Der nachgeschaltete Wechselrichter mit dreiphasigem Ausgang wird über die Zwischenkreisspannung versorgt, Bei kleinen und mittleren zu schaltenden Leistungen werden als Halbleiterschalter (Ventile) in der Regel IGBTs verwendet, für größere Leistungen GTO-Thyristoren (engl.: Gate Turn-Off Thyristor). Die Ventile werden von der Ansteuerung so getaktet, dass an den Klemmen jeder Motorwicklung eine pulsweitenmodulierte Spannung ansteht, dabei sind Betrag, Frequenz und Phasenwinkel der Spannungen einstellbar. Antiparallel zu jedem Ventil ist eine Freilaufdiode geschaltet, die den Wechselrichter u.a. vor auftretenden SelbstinduktionsSpannungen schützen, die während der Umschaltphasen der Ventile auftreten.

Da die Eingangsspannung für die B6-Wechselrichterbrücke eine weitgehend konstante Gleichspannung darstellt, wird der Wechselrichter auch als spannungseinprägender Wechselrichter oder U-Wechselrichter [190] bezeichnet. Abbildung 5.19 zeigt den prinzipiellen Aufbau des U-Wechselrichters.

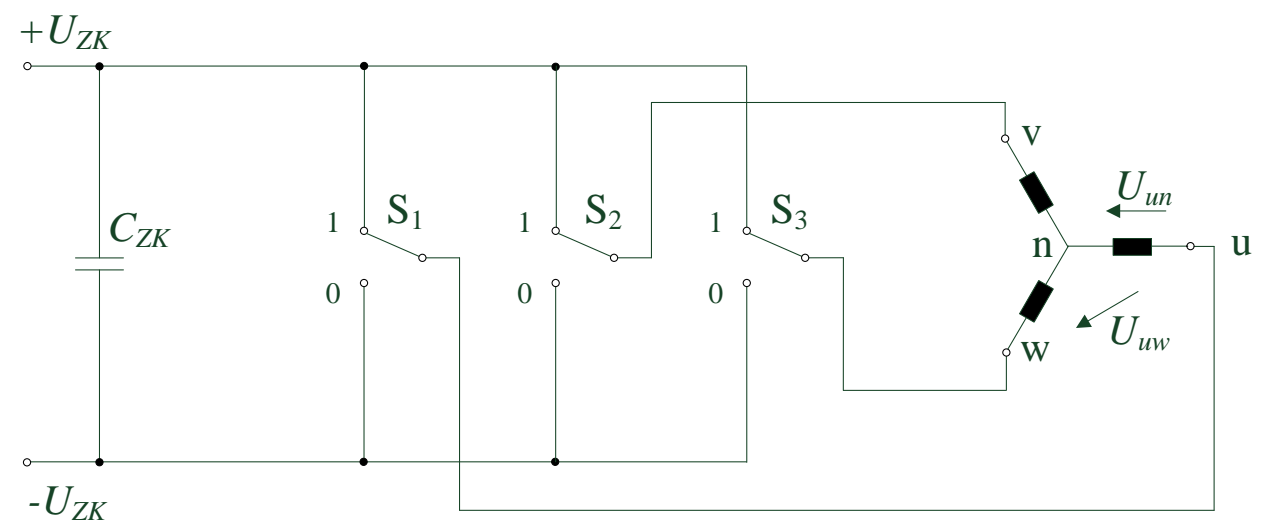

Abbildung 5.19.: Prinzipschaltbild des U-Wechselrichters [91].

Für die weitere Betrachtung soll gelten, dass die Spannungsbildung für den Motor so realisiert werden kann, als ob ideale Spannungsventile existieren. Im Ersatzschaltbild in Abbildung 5.19 wurden die Ventile ferner in jedem Zweig durch Wechselschalter ersetzt. Aus den drei Zweigen in Abbildung 5.19 mit je einem Wechselschalter lassen sich insgesamt acht mögliche logische Zustände $\left(2^{3}=8\right)$ ableiten. Somit können acht 
Raumzeiger gebildet werden. Die Phasenlagen der sechs Raumzeiger $\mathbf{u}_{1}, \mathbf{u}_{2}, \mathbf{u}_{3}, \mathbf{u}_{4}, \mathbf{u}_{5}$ und $\mathbf{u}_{6}$ sind jeweils um $60^{\circ}$ zueinander versetzt. Die Beträge der Phasenspannungen können die Werte $\pm 1 / 3 U_{Z K}$ bzw. $\pm 2 / 3 U_{Z K}$ (Tabelle 5.1) annehmen.

Tabelle 5.1.: Spannungsraumzeiger des Wechselrichters [91].

\begin{tabular}{|c|c|c|c|c|c|c|c|}
\hline & \multicolumn{2}{|c|}{ verkettete Spannungen } & \multicolumn{3}{|c|}{ Phasenspannung } & Raumzeiger \\
\hline $\mathrm{S}_{1}, \mathrm{~S}_{2}, \mathrm{~S}_{3}$ & $U_{u v}$ & $U_{v w}$ & $U_{w u}$ & $U_{u n}$ & $U_{v n}$ & $U_{w n}$ & $\mathbf{u}$ \\
\hline 000 & 0 & 0 & 0 & 0 & 0 & 0 & $\mathbf{u}_{0}=0$ \\
\hline 100 & $U_{Z K}$ & 0 & $-U_{Z K}$ & $\frac{2}{3} U_{Z K}$ & $-\frac{1}{3} U_{Z K}$ & $-\frac{1}{3} U_{Z K}$ & $\mathbf{u}_{1}=\frac{2}{3} U_{Z K} e^{j 0}$ \\
\hline 110 & 0 & $U_{Z K}$ & $-U_{Z K}$ & $\frac{1}{3} U_{Z K}$ & $\frac{1}{3} U_{Z K}$ & $-\frac{2}{3} U_{Z K}$ & $\mathbf{u}_{2}=\frac{2}{3} U_{Z K} e^{j \frac{\pi}{3}}$ \\
\hline 010 & $-U_{Z K}$ & $U_{Z K}$ & 0 & $-\frac{1}{3} U_{Z K}$ & $\frac{2}{3} U_{Z K}$ & $-\frac{1}{3} U_{Z K}$ & $\mathbf{u}_{3}=\frac{2}{3} U_{Z K} e^{j \frac{2 \pi}{3}}$ \\
\hline 011 & $-U_{Z K}$ & 0 & $U_{Z K}$ & $-\frac{2}{3} U_{Z K}$ & $\frac{1}{3} U_{Z K}$ & $\frac{1}{3} U_{Z K}$ & $\mathbf{u}_{4}=\frac{2}{3} U_{Z K} e^{j \pi}$ \\
\hline 101 & 0 & $-U_{Z K}$ & $U_{Z K}$ & $-\frac{1}{3} U_{Z K}$ & $-\frac{1}{3} U_{Z K}$ & $\frac{2}{3} U_{Z K}$ & $\mathbf{u}_{5}=\frac{2}{3} U_{Z K} e^{j \frac{4 \pi}{3}}$ \\
\hline 111 & 0 & 0 & 0 & 0 & 0 & 0 & $\mathbf{u}_{7}=0$ \\
\hline
\end{tabular}

Die verbleibenden zwei Raumzeiger bilden Nullvektoren. Die Schalterkombination $S_{1}=S_{2}=S_{3}=0$ erzeugt den Nullvektor $\mathbf{u}_{0}$ (alle Schalter liegen auf dem Potenzial $-U_{Z K}$ ), die Schalterkombination $S_{1}=S_{2}=S_{3}=1$ erzeugt den Nullvektor $\mathbf{u}_{7}$ (alle Schalter liegen auf dem Potenzial $\left.+U_{Z K}\right)$.

Abbildung 5.20 zeigt nochmals übersichtlich die Lage der möglichen Raumzeiger im $\alpha, \beta$-Koordinatensystem bzw. zum Wicklungssystem $u, v, w$ gemäß Tabelle 5.1 für die gewählten Schalterstellungen. Der Vektorraum wird dabei in vier Quadranten $Q_{1}, \cdots Q_{4}$ und 6 Sektoren aufgeteilt [144].

Für die spätere Regelung des Motors ist es wichtig, nicht nur die gezeigten acht Standardvektoren in Abbildung $5.20 \mathrm{zu}$ erzeugen, sondern einen beliebigen Statorspannungsvektor innerhalb des Vektorraumes. Da eine kontinuierliche verstellbare Stellgröße für die Amplitude und Phasenlage des umlaufenden Raumzeigers $\mathbf{u}_{s}$ durch das Stellglied nicht erzeugt werden kann, wird der Raumzeiger $\mathbf{u}_{s}$ durch Pulsen des 


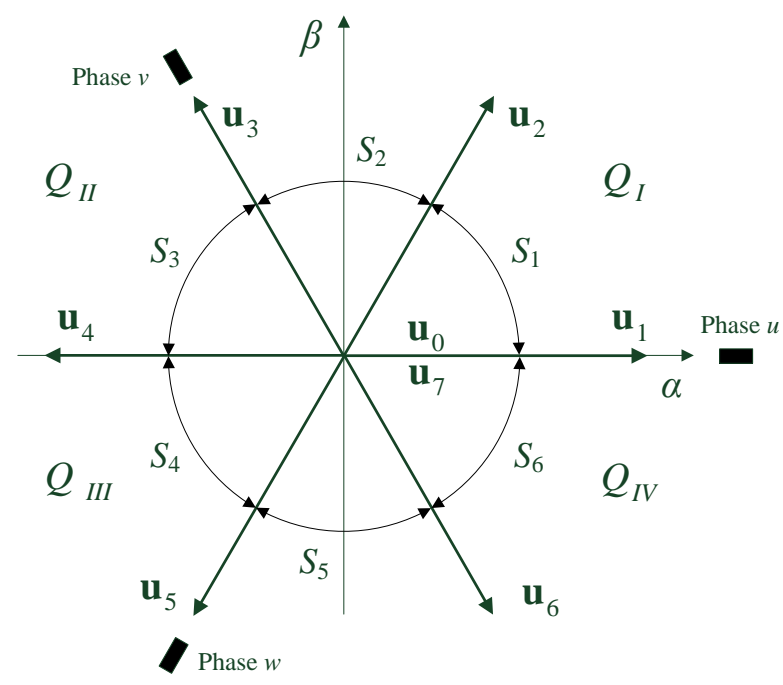

Abbildung 5.20.: Raumzeigerdarstellung der Ausgangsspannungen [91].

Wechselrichters, d.h. durch sinnvolles Umschalten zwischen den möglichen Standardvektoren gebildet - der gewünschte kontinuierliche Verlauf wird somit durch eine Pulsweitenmodulation angenähert.

Abbildung 5.21 zeigt das Prinzip der Vorgehensweise für einen Spannungsraumzeiger im Sektor 1. Soll ein Sollraumzeiger der Statorspannung $\mathbf{u}_{s}$ erzeugt werden, der

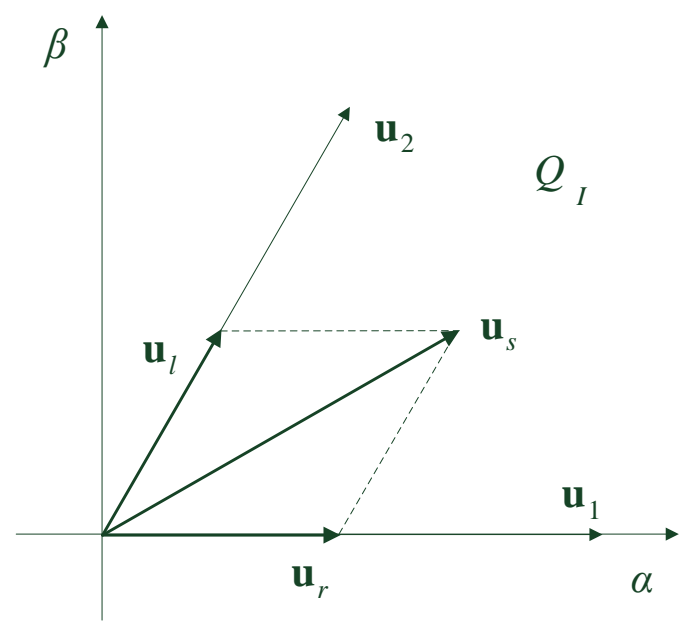

Abbildung 5.21.: Randvektoren des Spannungsraumzeigers [91].

sich innerhalb des ersten Sektors befindet, werden die Standardvektoren $\mathbf{u}_{1}, \mathbf{u}_{2}, \mathbf{u}_{7}$ und $\mathbf{u}_{0}$ so nacheinander angesteuert, dass sich der gewünschte Raumzeiger $\mathbf{u}_{s}$ bildet. Dabei ergibt sich $\mathbf{u}_{s}$ letztlich aus einer "quasi" vektoriellen Addition des rechten 
Randvektors $\mathbf{u}_{r}$ in Richtung des Standardvektors $\mathbf{u}_{1}$ und des linken Randvektors $\mathbf{u}_{l}$ in Richtung des Standardvektors $\mathbf{u}_{2}$. Der Ablauf gilt entsprechend auch für die anderen Sektoren, wenn ein Sollraumzeiger innerhalb eines Sektors erzeugt werden soll. Durch die Vorgehensweise ist es möglich, im ganzen Vektorraum einen Sollraumzeiger der Statorspannung $\mathbf{u}_{s}$ zu erzeugen. Bei der Vorgabe der Schaltfolge der Randvektoren muss berücksichtigt werden, dass die Schaltverluste des Wechselrichters möglichst gering sein sollen. Abbildung 5.22 zeigt den Ablauf für die Ansteuerung der Randvektoren für die Pulsperiode $T_{p}$ entsprechend Abbildung 5.21 für $\mathbf{u}_{s}$ im ersten Sektor.

\begin{tabular}{|c|c|c|c|c|c|c|c|c|}
\hline $\mathrm{S}_{1}, \mathrm{~S}_{2}, \mathrm{~S}_{3}$ & 000 & 100 & 110 & 111 & 110 & 100 & 000 & 111 \\
\hline \multicolumn{9}{|l|}{$u$} \\
\hline \multicolumn{9}{|l|}{$v$} \\
\hline \multicolumn{9}{|l|}{$w$} \\
\hline & $\mathbf{u}_{0}$ & $\mathbf{u}_{1}$ & $\mathbf{u}_{2}$ & $\mathbf{u}_{7}$ & $\mathbf{u}_{2}$ & $\mathbf{u}_{1}$ & $\mathbf{u}_{0}$ & $\mathbf{u}_{7}$ \\
\hline & $T_{0}$ & $T_{r}$ & $T_{l}$ & $T_{7}$ & $T_{l}$ & $T_{r}$ & $T_{0}$ & $T_{7}$ \\
\hline
\end{tabular}

Abbildung 5.22.: Bildung des Spannungsvektors [91].

In der ersten halben Pulsperiode $T_{p}{ }^{*}=T_{p} / 2$ wird $\mathbf{u}_{s}$ durch die Schaltzustände $\mathbf{u}_{0}$ $\Rightarrow \mathbf{u}_{1} \Rightarrow \mathbf{u}_{2} \Rightarrow \mathbf{u}_{7}$ zum ersten Mal generiert, in der zweiten halben Pulsperiode durch die Schaltzustände $\mathbf{u}_{7} \Rightarrow \mathbf{u}_{2} \Rightarrow \mathbf{u}_{1} \Rightarrow \mathbf{u}_{0}$. In Abhängigkeit von der konkreten Implementierung der Modulation sind auch andere Varianten für die Realisierung der Vektoren innerhalb der realen Pulsperiode $T_{p}$ möglich [144]. Wie die obere Zeile $\left(\mathrm{S}_{1}, \mathrm{~S}_{2}, \mathrm{~S}_{3}\right)$ und der Verlauf der Schaltsignale $(u, v, w)$ in Abbildung 5.22 zeigt, wird jeder Wechselrichterzweig innerhalb der Periode $T_{p}{ }^{*}$ nur einmal umgeschaltet, die Schaltverluste der Ventile sind deshalb minimal.

Da der Betrag und die Phasenlage des zu realisierenden Soll-Statorspannungsvektors $\mathbf{u}_{s}$ vom Stromregler vorgegeben werden, die Standardvektoren bzw. die Randvektoren aus Tabelle 5.1 bekannt sind, bleibt nur noch die Bestimmung der Schaltzeiten $T_{r}$ und $T_{l}$, Gln. (5.38) und (5.39), [144].

Die Vorgaben der Beträge des rechten Randvektors $\mathbf{u}_{r}$ und des linken Randvektors $\mathbf{u}_{l}$ liefert wie angesprochen der Stromregler. Für die verbleibende Zeitspanne $T_{p}{ }^{*}$ $\left(T_{r}+T_{l}\right)$, wie in Abbildung 5.22 veranschaulicht, wird der Nullvektor $\mathbf{u}_{0}$ oder $\mathbf{u}_{7}$ ausgegeben. Für die Bestimmung von $\mathbf{u}_{s}$ im ersten Sektor folgen somit Gl. (5.41), 
alternativ Gl. (5.42).

$$
\begin{aligned}
T_{r} & =\frac{\left|\mathbf{u}_{r}\right|}{\left|\mathbf{u}_{s}\right|_{\max }} T_{p}{ }^{*} . \\
T_{l} & =\frac{\left|\mathbf{u}_{l}\right|}{\left|\mathbf{u}_{s}\right|_{\max }} T_{p}{ }^{*} . \\
\left|\mathbf{u}_{s}\right|_{\max } & =\left|\mathbf{u}_{1}\right|=\cdots=\left|\mathbf{u}_{6}\right|=\frac{2}{3} U_{Z K} . \\
\mathbf{u}_{s}=\mathbf{u}_{r}+\mathbf{u}_{l}+\mathbf{u}_{0}= & \frac{T_{r}}{T_{p}{ }^{*}} \mathbf{u}_{1}+\frac{T_{l}}{T_{p}{ }^{*}} \mathbf{u}_{2}+\frac{T_{p}{ }^{*}-\left(T_{r}+T_{l}\right)}{T_{p}{ }^{*}} \mathbf{u}_{0} . \\
\mathbf{u}_{s}=\mathbf{u}_{r}+\mathbf{u}_{l}+\mathbf{u}_{7} & =\frac{T_{r}}{T_{p}{ }^{*}} \mathbf{u}_{1}+\frac{T_{l}}{T_{p}{ }^{*}} \mathbf{u}_{2}+\frac{T_{p}{ }^{*}-\left(T_{r}+T_{l}\right)}{T_{p}{ }^{*}} \mathbf{u}_{7} .
\end{aligned}
$$

Die Werte der Schaltzeiten $T_{r}$ und $T_{l}$ können entweder aus den gleichförmigen Komponenten $u_{d}, u_{q}$ im Rotorkoordinatensystem oder aus den sinusförmigen Komponenten $u_{\alpha}, u \beta$ im Statorkoordinatensystem gewonnen werden.

Tabelle 5.2 zeigt die Berechnung von $\mathbf{u}_{r}$ und $\mathbf{u}_{l}$ aus den statorfesten Komponenten von $u_{\alpha}$ und $u_{\beta}$ für die einzelnen Sektoren nach der Koordinatentransformation aus dem $d, q$-Koordinatensystem.

Die zu bestimmenden Werte von $\mathbf{u}_{r}$ und $\mathbf{u}_{l}$ entsprechend Tabelle 5.2 lassen sich vereinfachend durch die vier Terme $a_{1}, a_{2}, a_{3}$ und $a_{4}$ darstellen und berechnen, Gln. (5.43), (5.44), (5.45), (5.46).

$$
\begin{aligned}
& a_{1}=\left|u_{\alpha}\right|+\frac{1}{\sqrt{3}}\left|u_{\beta}\right| . \\
& a_{2}=\left|u_{\alpha}\right|-\frac{1}{\sqrt{3}}\left|u_{\beta}\right| . \\
& a_{3}=-\left|u_{\alpha}\right|+\frac{1}{\sqrt{3}}\left|u_{\beta}\right| . \\
& a_{4}=\quad \frac{2}{\sqrt{3}}\left|u_{\beta}\right| .
\end{aligned}
$$

Um die Phasenlage von $\mathbf{u}_{s}$ zu bestimmen, können in einem ersten Schritt die Vorzeichen von $u_{\alpha}$ und $u_{\beta}$ ausgewertet werden. Daraus lässt sich der Quadrant bestimmen, in dem sich der Spannungsvektor befindet (Abbildung 5.20). 
Tabelle 5.2.: Randkomponenten des Spannungsvektors [181].

\begin{tabular}{|c|c|c|c|}
\hline \multicolumn{2}{|c|}{} & $\left|\mathbf{u}_{r}\right|$ & $\left|\mathbf{u}_{l}\right|$ \\
\hline$S_{1}$ & $Q_{I}$ & $\left|u_{\alpha}\right|-\frac{1}{\sqrt{3}}\left|u_{\beta}\right|$ & $\frac{2}{\sqrt{3}}\left|u_{\beta}\right|$ \\
\hline$S_{2}$ & $Q_{I}$ & $\left|u_{\alpha}\right|+\frac{1}{\sqrt{3}}\left|u_{\beta}\right|$ & $-\left|u_{\alpha}\right|+\frac{1}{\sqrt{3}}\left|u_{\beta}\right|$ \\
\hline$S_{2}$ & $Q_{I I}$ & $-\left|u_{\alpha}\right|+\frac{1}{\sqrt{3}}\left|u_{\beta}\right|$ & $\left|u_{\alpha}\right|+\frac{1}{\sqrt{3}}\left|u_{\beta}\right|$ \\
\hline$S_{3}$ & $Q_{I I}$ & $\frac{2}{\sqrt{3}}\left|u_{\beta}\right|$ & $\left|u_{\alpha}\right|-\frac{1}{\sqrt{3}}\left|u_{\beta}\right|$ \\
\hline$S_{4}$ & $Q_{I I I}$ & $\left|u_{\alpha}\right|-\frac{1}{\sqrt{3}}\left|u_{\beta}\right|$ & $\frac{2}{\sqrt{3}}\left|U_{\beta}\right|$ \\
\hline$S_{5}$ & $Q_{I I I}$ & $\left|u_{\alpha}\right|+\frac{1}{\sqrt{3}}\left|u_{\beta}\right|$ & $-\left|u_{\alpha}\right|+\frac{1}{\sqrt{3}}\left|u_{\beta}\right|$ \\
\hline$S_{5}$ & $Q_{I V}$ & $-\left|u_{\alpha}\right|+\frac{1}{\sqrt{3}}\left|u_{\beta}\right|$ & $\left|u_{\alpha}\right|+\frac{1}{\sqrt{3}}\left|u_{\beta}\right|$ \\
\hline$S_{6}$ & $Q_{I V}$ & $\frac{2}{\sqrt{3}}\left|u_{\beta}\right|$ & $\left|u_{\alpha}\right|-\frac{1}{\sqrt{3}}\left|u_{\beta}\right|$ \\
\hline
\end{tabular}

\subsubsection{Aufbau und Realisierung einer neuen Regelungsplattform innerhalb einer Embedded-System Umgebung}

Der wohl wichtigste Unterschied zwischen einem FPGA und einem Mikrocontroller ist die Möglichkeit des FPGAs, Daten parallel zu verarbeiten - Mikrocontroller arbeiten bekannterweise stets sequenziell. Beim FPGA ist es möglich, durch den Aufbau von parallel arbeitenden Prozessen flexiblere und leistungsfähigere Strukturen aufzubauen. Allerdings ist der Umgang mit der Systemumgebung und der Synthetisierung des FPGAs in der Regel komplexer als beim Mikrocontroller. Auf den grundsätzlichen Aufbau und die Beschreibung der Grundelemente eines FPGAs soll an der Stelle nicht näher eingegangen werden. Umfangreiche Fachliteratur findet sich in [136], [169], [170].

Die nachfolgenden Abschnitte beschreiben die Umsetzung der FOC innerhalb einer SoPC-basierten Embedded System Plattform. Das Motorsteuerkonzept wurde auf der im Anhang 5.2 beschriebenen Referenzplattform umgesetzt. Zuerst soll allerdings auf die Realisierung der Motorstrommessung eingegangen werden. Eine exakte, schnelle und störungsfreie Strommessung ist ein elementarer Bestandteil für die Umsetzung der FOC. 


\section{Sigma-Delta-Wandler}

Wichtig für den dynamischen Betrieb des Drehstrommotors in der FOC ist eine schnelle und präzise Messung der Motorströme. Generell werden im Markt unterschiedliche Sensoren für die Strommessung angeboten. Dabei wird unterschieden in kapazitive, induktive und optische Übertragungsverfahren. Klassisch werden zur Bestimmung der Motorströme Shunts [152] oder Hall-Sensoren [165] eingesetzt. Die gemessenen analogen Signalspannungen werden dann über A/D-Wandler eingelesen. Die A/D-Wandler sind in der Regel bereits im modifizierten Mikrocontroller der Hersteller integriert. Bei einer solchen technischen Ausführung wird vor dem A/DWandler ein Tiefpassfilter geschaltet, welcher vor allem die durch die hohe Flankenanstiegsgeschwindigkeit der Ausgangsspannung eingekoppelten Störsignale reduziert.

Sigma-Delta-Wandler ( $\Sigma \Delta$-Wandler), in der Literatur auch als Delta-Sigma-Wandler bezeichnet, werden gleichfalls in einer Vielzahl von Anwendungen zur Analog-DigitalWandlung elektrischer Signale eingesetzt. $\Sigma \Delta$-Wandler unterscheiden sich dabei vom Aufbau her grundsätzlich zu anderen klassischen Wandlern, die z.B. nach dem Parallel-, Wäge- oder Zählverfahren arbeiten [217]. Wichtige Merkmale des $\Sigma \Delta$-Wandlers sind das Oversampling (Überabtastung), Noise Shaping (Rauschformung oder auch Störspektrumsformung) und die zugehörige digitale Filterung [40].

Während des Abtastvorganges wird ein wert- und zeitkontinuierliches (analoges) Signal in ein wert- und zeitdiskretes (digitales) Signal überführt. Dabei entsteht durch die begrenzte Auflösung des Wandlers eine Verzerrung des Signals. Betrachtet man einen klassischen idealen A/D-Wandler mit $n$-Bit Auflösung ist der sogenannte Quantisierungsfehler [73], eine zufällige Größe im Bereich von max. $\pm 1 / 2$ LSB (engl.: Least Significant Bit), analytisch berechenbar. Dabei geht man von der Annahme aus, dass das Eingangssignal stationär und mittelwertfrei ist. Geht man weiter davon aus, dass der Fehler stetig gleichverteilt und unkorreliert zum Eingangssignal ist, kann der Quantisierungsfehler dann als stochastischer Prozess dargestellt werden dem sogenannten Quantisierungsrauschen.

Die Bewertung des Quantisierungsrauschens ist durch die Angabe des Signal-RauschVerhältnisses (SNR, engl.: Signal-to-Noise Ratio) möglich. Das SNR beschreibt dabei das Verhältnis der mittleren Leistung des Nutzsignals zur mittleren Rauschleistung des Störsignals. Für einen idealen $n$-Bit A/D-Wandler berechnet sich das SNR gemäß Gl. (5.47) [73].

$$
S N R=(6,02 n+1,76) d B .
$$

Um ein ausreichend großes Signal-Rausch-Verhältnis zu erreichen, muss die Anzahl der Bit bzw. die Auflösung des A/D-Wandlers entsprechend erhöht werden. Jedes 
zusätzliche Bit verbessert das SNR und somit die Genauigkeit der Signalreproduktion um $6 d B$. Für die Abtastfrequenz $f_{S}$ gilt dabei, dass sie dem Nyquist-Kriterium genügen muss [92], d.h. $f_{S}$ muss mindestens doppelt so groß sein wie die höchste vorkommende Frequenz im Eingangssignal. Jede weitere Erhöhung der Abtastfrequenz $f_{S}$ führt zu einer Überabtastung (OSR, engl.: Oversampling rate) des Signals. Die Überabtastrate wird gemäß Gl. (5.48) beschrieben [73]. $B$ steht für die Bandbreite des Eingangssignals.

$$
O S R=\frac{f_{S}}{2 B}
$$

Positiv wirkt sich aus, dass sich durch die Erhöhung der Abtastfrequenz die Amplitude des Rauschanteiles verkleinert. Das SNR bleibt zwar gleich, die Rauschenergie verteilt sich aber auf einen größeren Frequenzbereich.

\section{Sigma-Delta-Modulator}

$\Sigma \Delta$-Wandler sind so aufgebaut, dass sie den beschriebenen Effekt der Rauschformung nutzen [73]. In Abbildung 5.23 ist das prinzipielle Blockschaltbild eines $\Sigma \Delta$ Modulators 1. Ordnung dargestellt. Wird die Schaltung am Ausgang noch um ein Filternetzwerk und einen Dezimierer erweitert, entsteht durch die gesamte Anordnung ein $\Sigma \Delta$-Wandler.

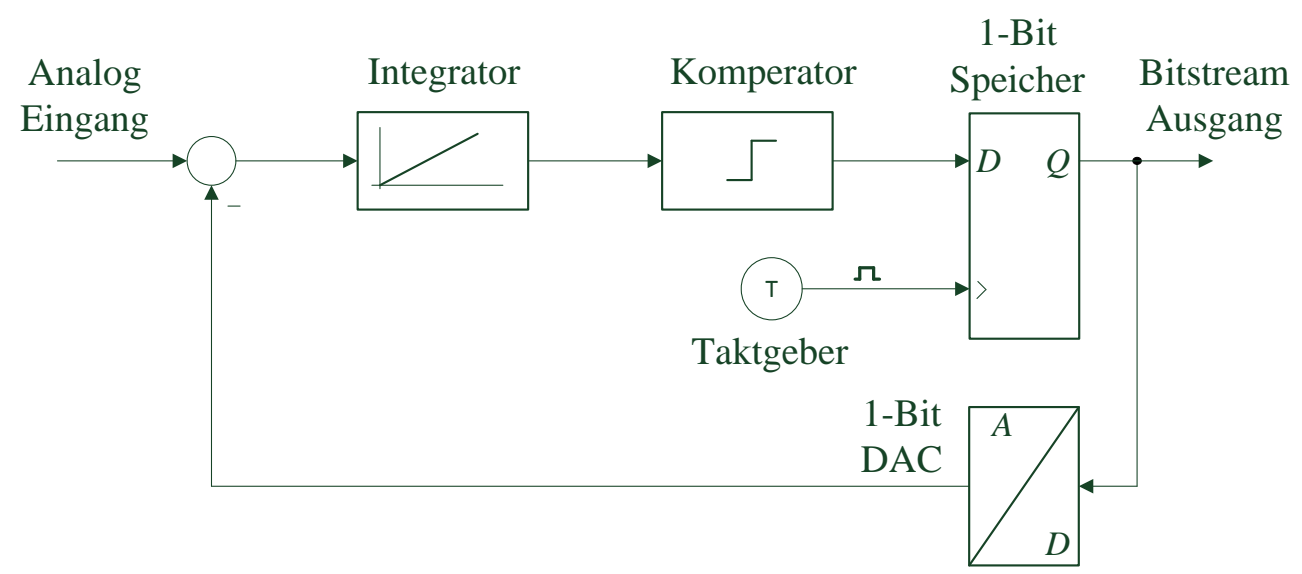

Abbildung 5.23.: Blockschaltbild eines $\Sigma \Delta$-Modulator 1. Ordnung [21].

Der Kern des Modulators besteht aus dem analogen Integrator, einem Komparator (1-Bit A/D-Wandler bzw. 1-Bit-Quantisierer) und einem 1-Bit-Speicher, der mit einer deutlich höheren Abtastrate (OSR) getaktet wird, als es die Signalbandbreite des Eingangssignals aufgrund des Abtasttheorems mindestens erfordert. 
Im Rückkopplungszweig wird das gepulste Signal, das am Ausgang ansteht, über einen 1-Bit D/A-Wandler wieder zurück auf den Differenzeingang geschaltet. Der D/A-Wandler funktioniert in dem Zusammenhang wie ein Schalter. Abhängig vom binären Ausgangszustand schaltet der D/A-Wandler eine positive oder negative Referenzspannung auf den Differenzeingang.

Das Differenzsignal aus Eingangssignal und rückgekoppeltem Ausgangsignal steht nun am Integratoreingang an. Der Komparator entscheidet in Abhängigkeit des Ausgangssignals des Integrators, ob das Signal größer oder kleiner als die am Komparator eingestellte beliebige Schwellwertgrenze ist.

Der 1-Bit-Speicher schaltet das binäre Signal vom Komparator bei jedem Takt weiter zum Bitstream Ausgang. Bei jedem Durchschreiten der Komparatorschwelle in positiver Richtung wird dabei der 1-Bit-Speicher auf den Wert 1 gesetzt - beim negativen Durchlauf der Komparatorschwelle auf den Wert 0. Dabei ist es auch die Aufgabe des D/A-Wandlers, das Ausgangssignal des Integrators nahe an der Referenzschwelle des Komparators zu halten.

Durch die hohe Überabtastung zwischen zwei Abtastzeitpunkten treten nur geringe Signalveränderungen auf, wodurch die Verwendung eines einfachen 1-Bit A/DWandlers möglich ist. Verändert sich das Eingangssignal stark, erzeugt der Komparator eine steigende Anzahl von 0- und 1-Zuständen im Bitstream. Der Mittelwert des erzeugten Bitstreams entspricht dabei dem Wert des Eingangssignales.

Im Aussteuerbereich wird die positive bzw. negative Referenzspannung durch die hohe Signaleingangsspannung kompensiert, d.h. der Integratorausgang ändert sich nur langsam, was dazu führt, dass sich in einem solchem Fall das Bitstream-Muster weniger häufig ändert.

Der eigentliche Vorteil des Modulators besteht darin, dass durch die hohe Überabtastung und durch das Summieren der im Signal enthaltenen Störspannungen (Fehlersignal) der Integrator als Tiefpass für das Eingangssignal und als Hochpass für das Quantisierungsrauschen wirkt. Der Anteil des Quantisierungsrauschens, der durch die Überabtastung weit oberhalb der Signalbandbreite $f_{B}$ verschoben wurde (Noise Shaping), kann mit einem nachfolgenden digitalen Tiefpassfilter einfacher ausgefiltert werden, der Effektivwert des Rauschens verkleinert sich dadurch.

$\Sigma \Delta$-Modulatoren 1. Ordnung erreichen eine $9 d B$ SNR-Verbesserung pro Verdopplung des Oversamplings. Allerdings erzeugen Modulatoren 1. Ordnung ausgeprägte Frequenzen im Spektrum, die unerwünscht sind [21]. Werden mehrere Summier- und Integrationsstufen im Modulator in Reihe geschaltet, resultieren daraus $\Sigma \Delta$-Modulatoren höherer Ordnung. So bringt ein Modulator 2. Ordnung bereits eine SNR-Verbesserung von ca. $15 \mathrm{~dB}$ bei Verdopplung der Oversampling-Rate (vgl. Abbildung 5.24). Die Rauschübertragungsfunktion $Q(f)$ eines $\Sigma \Delta$-Modulators $K_{m}$-ter Ordnung lässt sich nach Gl. (5.49) beschreiben [146]. 


$$
Q(f)=\frac{U_{L S B}}{\sqrt{12 f_{S}}}\left(2 \sin \left(\pi \frac{f}{f_{S}}\right)\right)^{K_{m}}
$$

Die Rauschspannung im Bereich der Bandbreite $B$ ergibt sich dann aus der Gl. (5.49) und Gl. (5.50).

$$
\begin{aligned}
U_{r m s} & =\sqrt{2 \int_{0}^{B} U^{2}(f) d f} . \\
U_{Q, r m s} & =\sqrt{2 \int_{0}^{B} \frac{U_{L S B}^{2}}{12 f_{S}}\left(2 \sin \left(\pi \frac{f}{f_{S}}\right)\right)^{2 K_{m}} d f} .
\end{aligned}
$$

Nach der Auflösung des Integrals in Gl. (5.51) kann für verschiedene $\Sigma \Delta$-Modulatoren höherer Ordnung, in Verbindung mit einem idealen Tiefpassfilter, die Funktion für das ideale SNR angeben werden, Gl. (5.52) [146].

$$
S N R_{I D E A L}=\left[6,02 n+1,76-20 \log \left[\frac{\pi^{K_{m}}}{\sqrt{2 K_{m}+1}}\right]+\left(20 K_{m}+10\right) \log O S R\right] d B .
$$

Abbildung 5.24 zeigt den Einfluss der Überabtastung (OSR) bei verschiedenen $\Sigma \Delta$ Modulatoren 1. bis 3. Ordnung auf das zu erreichende SNR in Verbindung mit einem idealen Tiefpassfilter.

Beträgt das SNR bei Verwendung eines $\Sigma \Delta$-Modulators 2. Ordnung und einer Überabtastung von $\mathrm{M}=32,70 \mathrm{~dB}$, liegt der SNR-Gewinn bei Verwendung eines $\Sigma \Delta$ Modulatoren 3. Ordnung, bei gleicher Überabtastung, bereits bei ca. $92 d B$. Je nach Bandbreite des Eingangssignals kann durch die Wahl der Taktrate und durch die Wahl der Ordnung des Modulators die Qualität des Ausgangssignals für den idealen Wandler ermittelt werden.

\section{Digitaler Dezimierungsfilter}

Der komplette Aufbau des $\Sigma \Delta$-Wandlers ist in Abbildung 5.25 dargestellt. Er besteht aus dem bereits beschriebenen $\Sigma \Delta$-Modulator und dem Filteranteil, der im FPGA realisiert ist. Der hochfrequente Bitstream am Ausgang des $\Sigma \Delta$-Modulators, der im Megahertz-Bereich überabgetastet wurde, ist für die Verarbeitungsgeschwindigkeit des FPGAs unkritisch bzw. die Umsetzung der digitalen Filterfunktion lässt sich im 


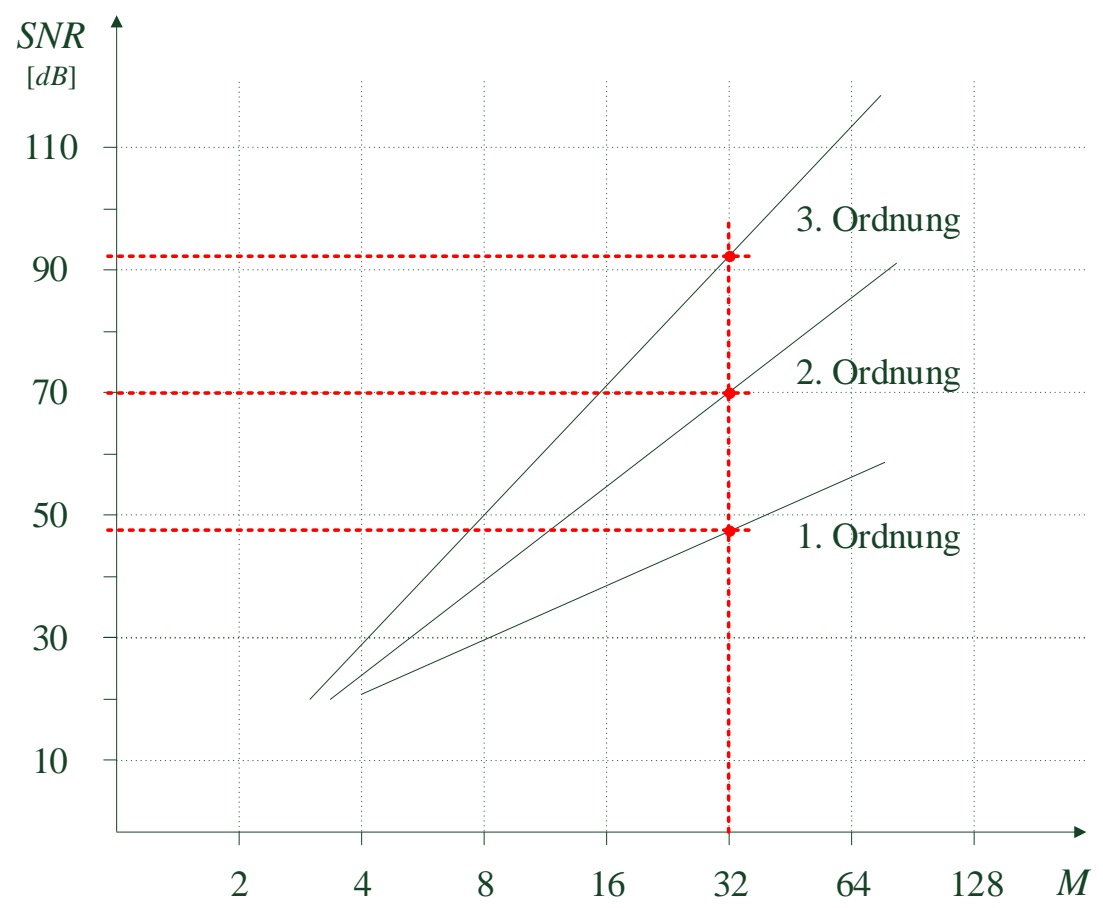

Abbildung 5.24.: Abhängigkeiten des $\Sigma \Delta$-Modulator von OSR und Ordnungsgrad (Darstellung der Ergebnisse aus Gl. (5.52)).

FPGA einfach realisieren. Das Filter selbst besteht aus zwei Teilen, dem eigentlichen Tiefpassfilter und einem Dezimierer.

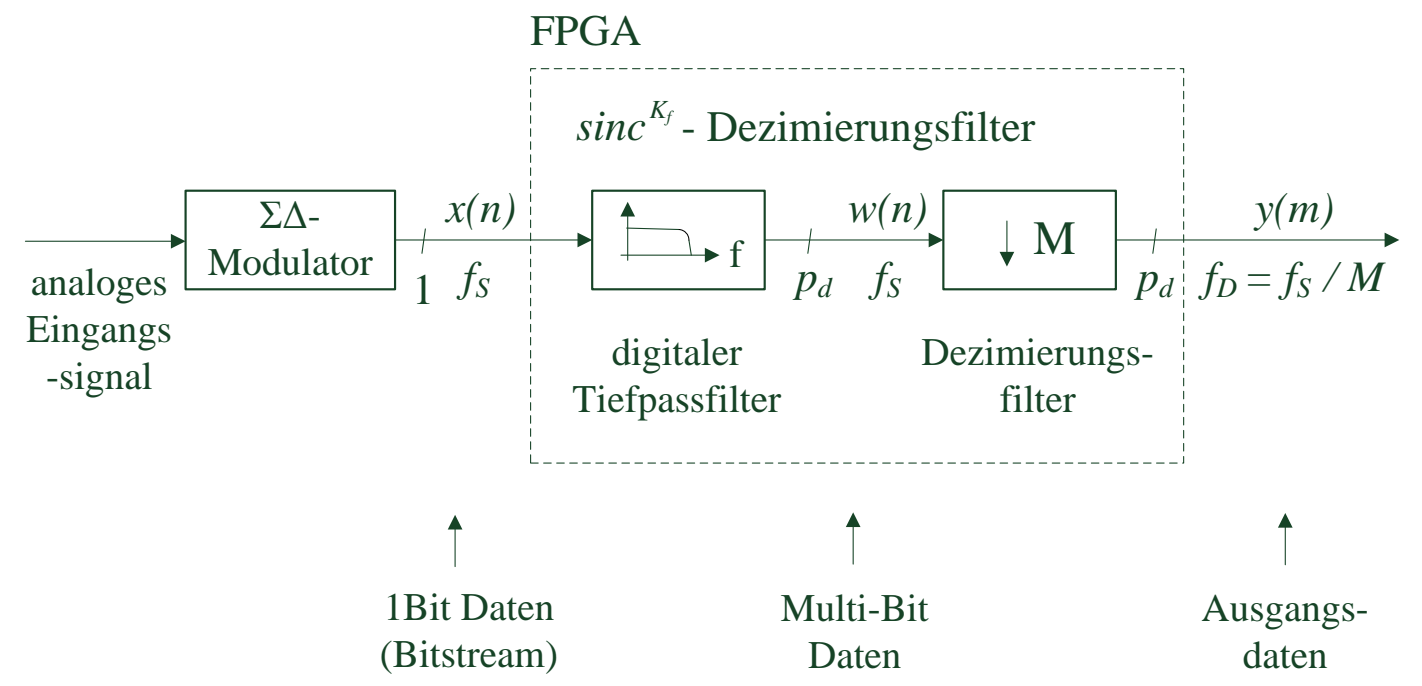

Abbildung 5.25.: $\Sigma \Delta$-Wandler: Modulator mit Digitalteil [146].

Die Funktion des Tiefpassfilters ist es, die hochfrequenten Anteile aus dem Quan- 
tisierungsrauschen bzw. des eigentlichen Wandlungsrauschens zu unterdrücken und aus dem 1-Bit Datenstrom am Eingang des Filters ein $p_{d}$-Bit breites Datenwort mit hohem SNR zu generieren. Die Bitbreite $p_{d}$ des Datenwortes berechnet sich gemäß Gl. (5.53) [146].

$$
p_{d}=K_{f} \log _{2} M
$$

Dabei ist die Bitbreite eine Funktion des Dezimierungsfaktors $M$ und der Ordnung des Filters $K_{f}$. Am Ausgang des digitalen Tiefpassfilters steht dann das rekonstruierte aber noch überabgetastete Eingangssignal zur Verfügung. Nach dem NyquistKriterium sind alle Informationen des abgetasteten Eingangssignals rekonstruierbar, wenn die Abtastfrequenz $f_{S}$ mindestens die doppelte Bandbreite des Eingangssignals besitzt. Durch das Dezimierungsfilter kann somit die hohe Datenrate aus der Überabtastung auf eine gewünschte niedrigere weiterzuverarbeitende Datenrate reduziert werden, ohne dass ein Informationsverlust entsteht. Für die praktische Realisierung folgt daraus, dass zur Reduzierung der Datenrate nur jeder $M$-te Ausgabewert des digitalen Filters ausgelesen bzw. weiterverarbeitet wird - wobei für den Dezimierungsfaktor $M$ gilt: $M \leq O S R$ [146].

Eine Möglichkeit, das Filter im FPGA zu implementieren, ist die Bildung des gleitenden Mittelwerts über dem Ausgangssignal des Modulators. Gl. (5.54) beschreibt die Übertragungsfunktion im $z$-Bereich für die Mittelwertbildung $K_{f}$-ter Ordnung. $M$ steht für den Dezimierungsfaktor, bezogen auf $M$ Abtastwerte [146].

$$
H(z)=\left[\frac{1}{M} \frac{\left(1-z^{-M}\right)}{\left(1-z^{-1}\right)}\right]^{K_{f}}
$$

Das erreichbare SNR ist in erster Linie von der Ordnung des $\Sigma \Delta$-Wandlers abhängig (vgl. Abbildung 5.24). Damit sich der Signal-Rausch-Abstand im Digitalteil nicht verschlechtert, muss die Ordnung des Filters $K_{f}=K_{m}+1$ aufweisen [35], [146]. $K_{m}$ bezeichnet die Ordnung des $\Sigma \Delta$-Modulators.

Eine hardwarenahe Implementierung des Filters lässt sich im FPGA einfach realisieren. Digitale Multiplizierer sind nicht notwendig. Für die Umsetzung werden nur Integrations- und Differentiationsbausteine benötigt. Abbildung 5.26 zeigt die Umsetzung des Filters 3. Ordnung.

Wird in Gl. (5.54) z durch $e^{-j}$ substituiert, folgt Gl. (5.55) [146].

$$
\left|H\left(e^{j w}\right)\right|=\left(\frac{1}{M} \frac{\sin (\omega M / 2)}{\sin (\omega / 2)}\right)^{K_{f}} \text { mit } \omega=2 \pi \frac{f}{f_{S}}
$$



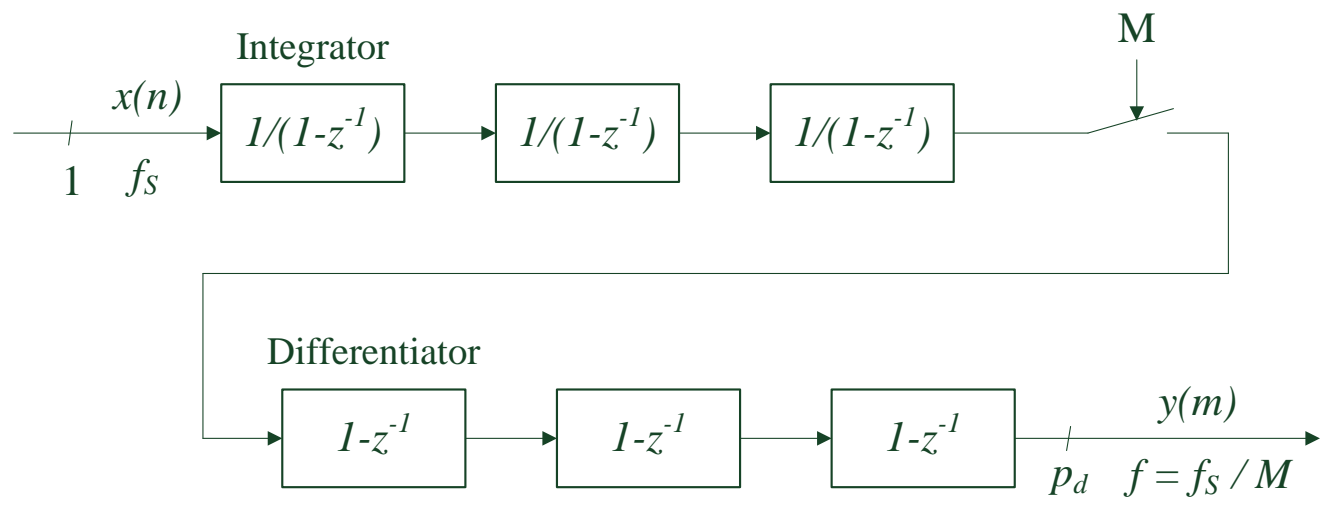

Abbildung 5.26.: Digitales Filter 3. Ordnung [146].

Abbildung 5.27 zeigt den Verlauf für den Betrag des Frequenzganges eines Filters 3. Ordnung gemäß Gl. (5.55) mit dem Dezimierungsfaktor $M=16$. Die Filterfunktion besitzt ,spektrale Nullstellen" an den Vielfachen der Dezimiererausgangsfrequenz $f_{D}$. Die Dezimiererausgangsfrequenz berechnet sich gemäß Gl. (5.56) [146]. $f_{S}$ bezeichnet wieder die Abtastfrequenz, $M$ den Dezimierungsfaktor.

$$
f_{D}=\frac{f_{S}}{M}
$$

Die Abbildung 5.27 zeigt den typischen Verlauf eines Notch-Filters - er wird auch als Kerbfilter bezeichnet. Notch-Filter sind Bandsperren, sie zeichnen sich aus durch eine hohe Dämpfung innerhalb eines sehr schmales Frequenzbereiches. Notch-Filter eignen sich für Anwendungen, bei denen bekannte Störungen bzw. zu unterdrückende Frequenzen bekannt sind.

Die Position der Bandsperre ist im konkreten Fall direkt abhängig von der AusgangsDatenrate, so können z.B. Einflüsse der Netzfrequenz unterdrückt werden. Über die gewählte Datenrate im Dezimierer $f_{D}$ kann die Notch-Eigenschaft des Filters somit berücksichtigt werden [146].

\section{SoPC-basiertes Embedded System}

Wie eingangs in Abschnitt 5.1 bereits beschrieben, stellen Soft-Cores veränderbare bzw. parametrierbare Modelle eines Mikroprozessors innerhalb eines FPGA-Designs dar. Sie werden in der Regel als (V)HDL Code vom Hersteller angeboten und bilden meist eine 32 Bit RISC Harvard Prozessorstruktur (engl.: Reduced Instruction Set Compute) ab. Anbieter solcher Soft-Core-Prozessorkerne sind z.B. die Firmen Xilinx 


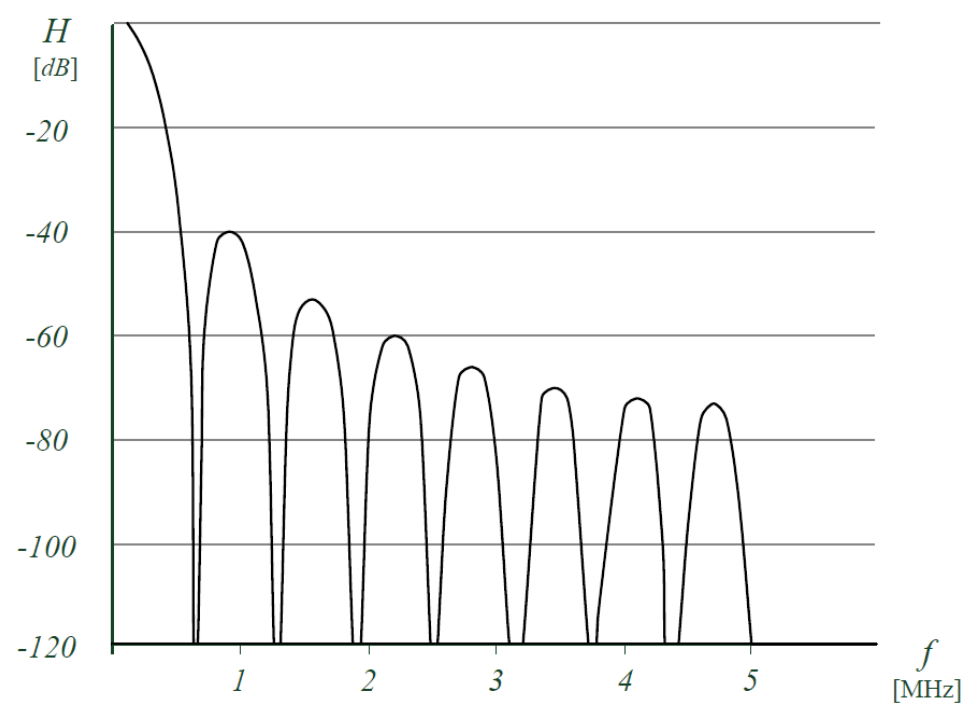

Abbildung 5.27.: Betrag der Filterfunktion 3. Ordnung, $M=16$ [146].

(MicroBlaze [234]), IBM (PowerPC 440 [81]), MIPS (Mips32 [137]) und Altera (NI$O S[8])$. Die aufgeführten Modelle sind in ihrer Struktur vergleichbar, unterscheiden sich aber in ihrer Erweiterbar- und Anpassbarkeit.

Abbildung 5.28 zeigt die prinzipielle Struktur des Aufbaus eines FPGAs mit SoftCore und IP-Blöcken. Ein solches System wird auch als SoPC (engl.: System on a Programmable Chip) oder SoPC basiertes Embedded System bezeichnet. Ein SoPC verweist darauf, dass die umgesetzten Funktionen und Algorithmen in einem wieder programmierbaren Logikbaustein realisiert sind. Durch die Integration des Soft-Cores im FPGA kann somit nicht nur die Hardware des FPGAs angepasst werden, sondern auch ein hinterlegtes Software-Programm im Mikroprozessor.

Der Soft-Core selbst besteht aus einer ALU (engl.: Arithmetic Logic Unit), einer FPU (engl.: Floating Point Unit), dem Interruptcontroller (IRQ) und verschiedenen Daten- und Befehlscaches sowie verschiedenen Speicherregistern. Die Komponenten werden in der Regel über ein On-Chip Bussystems miteinander verbunden.

Bei der konkreten Auswahl eines geeigneten Soft-Cores sind verschiedene Faktoren zu berücksichtigen, wie z.B. die maximale Größe des Befehls- und Datencaches, die Anzahl der GP-Register (engl.: General Purpose) oder die Möglichkeit, den Befehlssatz zu erweitern. Auch sollte geprüft werden, ob ein Co-Prozessor unterstützt wird. Die Entwicklungsumgebung sollte benutzerfreundlich sein, die Entwicklungswerkzeuge leistungsfähig und praktikabel. Die Soft-Core Hersteller wie auch Drittanbieter [175], [223] bieten fertige IP-Core Lösungen für die Peripherie- und Mikroprozessoren/DSPEinheiten am Markt an. Hier stellt sich die Frage der Kompatibilität zum vorhan- 


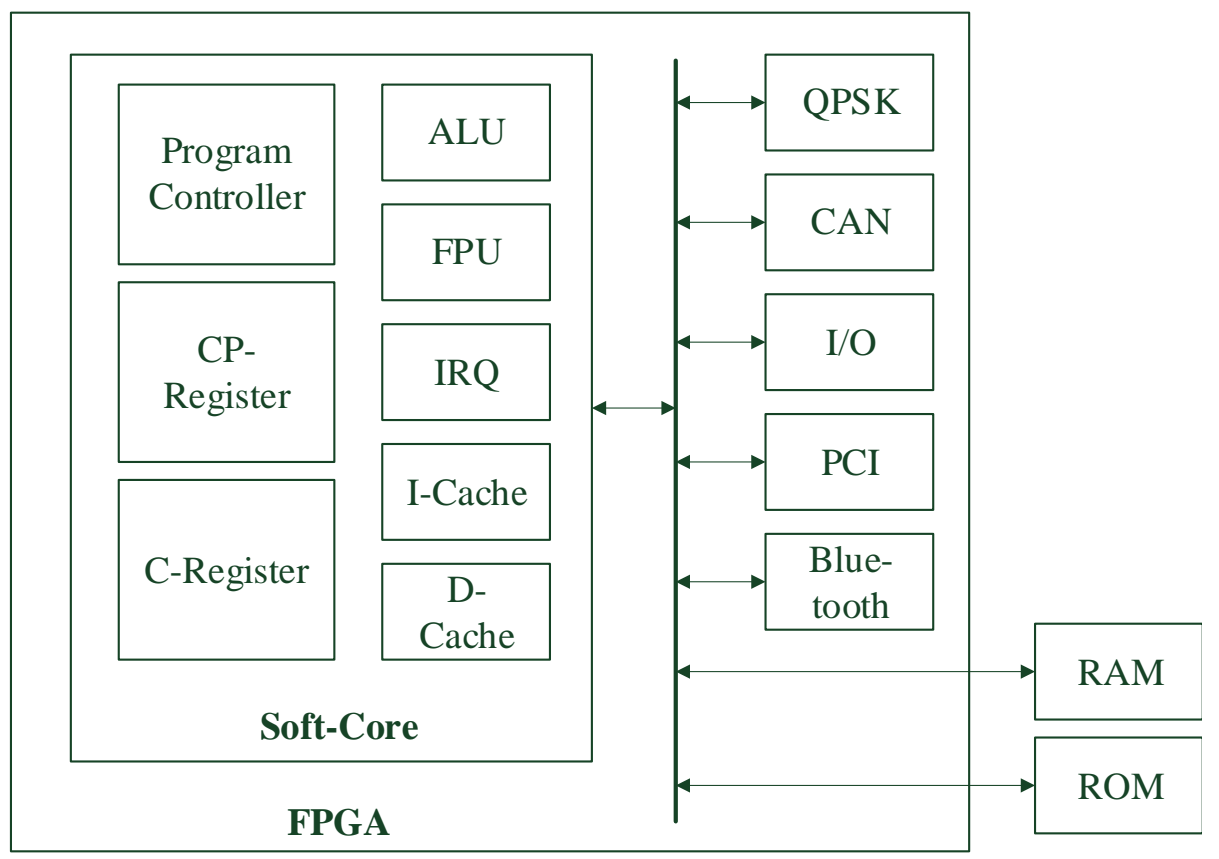

Abbildung 5.28.: 32 Bit RISCS HDL-Struktur auf einem SoPC [6].

denen bzw. zu dem erstellenden Modell und zum verwendeten Systembus. Neben der eigenen Entwicklung von IP-Cores besteht auch die Möglichkeit, auf sogenannte Opencore-Modelle [148] zuzugreifen - vergleichbar mit der bekannten OpensourceSoftware.

Bei den internen Bussystemen sind vier Architekturen [158] verbreitet: Fa. IBM ( $C O-$ RECONNECT [82]), Fa. ARM (AMBA [13]), Fa. Silicore Corporation (WISHBONE [76]) und das Bussystem der Fa. Altera (ASF, engl.: Avalon Switch Fabric [9]). Je nach Hersteller werden unterschiedliche Busse mit abgestufter Performance bzw. Geschwindigkeit angeboten oder nur ein Bus für verschiedene Geschwindigkeiten. Weitere Unterscheidungsmerkmale sind bei einigen Herstellern, dass die Datenbusbreite konfigurierbar ist, bei anderen ist sie auf 32 Bit begrenzt. Die meisten Bussysteme bieten Multi-Master-Multi-Slave Betrieb, wobei nur beim ASF-Bus ein simultaner Multi-Master Betrieb (Simultaneous Multi-Mastering) möglich ist, d.h. es können gleichzeitig Operationen von mehreren Mastern auf dem Bus erfolgen.

\section{Soft-Core}

Für die Umsetzung der FPGA-basierten Regelung wurde als Plattform die Architektur der Fa. Altera gewählt. Die Plattform bietet die Möglichkeit eines skalierbaren hardwaretechnischen Aufbaus und stellt leistungsfähige Entwicklungswerkzeuge zur 
Verfügung. Auch die Möglichkeit, durch die Verwendung des ASF-Busses einen simultanen Multi-Master Betrieb zu realisieren, steigert die Leistungsfähigkeit und Möglichkeiten bei der Umsetzung der Anwendung. Als FPGA-Plattform wurde ein Cyclone III gewählt [5], er bietet durch die Anzahl seiner Logikelemente (vgl. Abschnitt 5.2.2) eine ausreichende Performance.

Der im Cyclone III verwendete Soft-Core-Mikroprozesssor trägt die Bezeichnung $\mathrm{Ni}$ os II und wird vom gleichen Hersteller wie der FPGA als kommerzielles Produkt vertrieben [8]. Das im Nios II hinterlegte Anwenderprogramm koordiniert dabei im wesentlichen die Steuer- und Regelungsfunktionen mit den Aufrufen der IP-Blöcke für die Motorregelung. Der Nios II Kern selbst ist als 32 RISC-Prozessor aufgebaut.

Um ein komplettes, auf programmierbarer Logik basierendes System im FPGA aufzubauen, stellt die Fa. Altera innerhalb einer Entwicklungsumgebung verschiedene Tools zur Verfügung. Die Abbildung 5.29 gibt einen Überblick über die in der Arbeit verwendeten Entwicklungswerkzeuge. Quartus II bezeichnet die Entwicklungsumge-

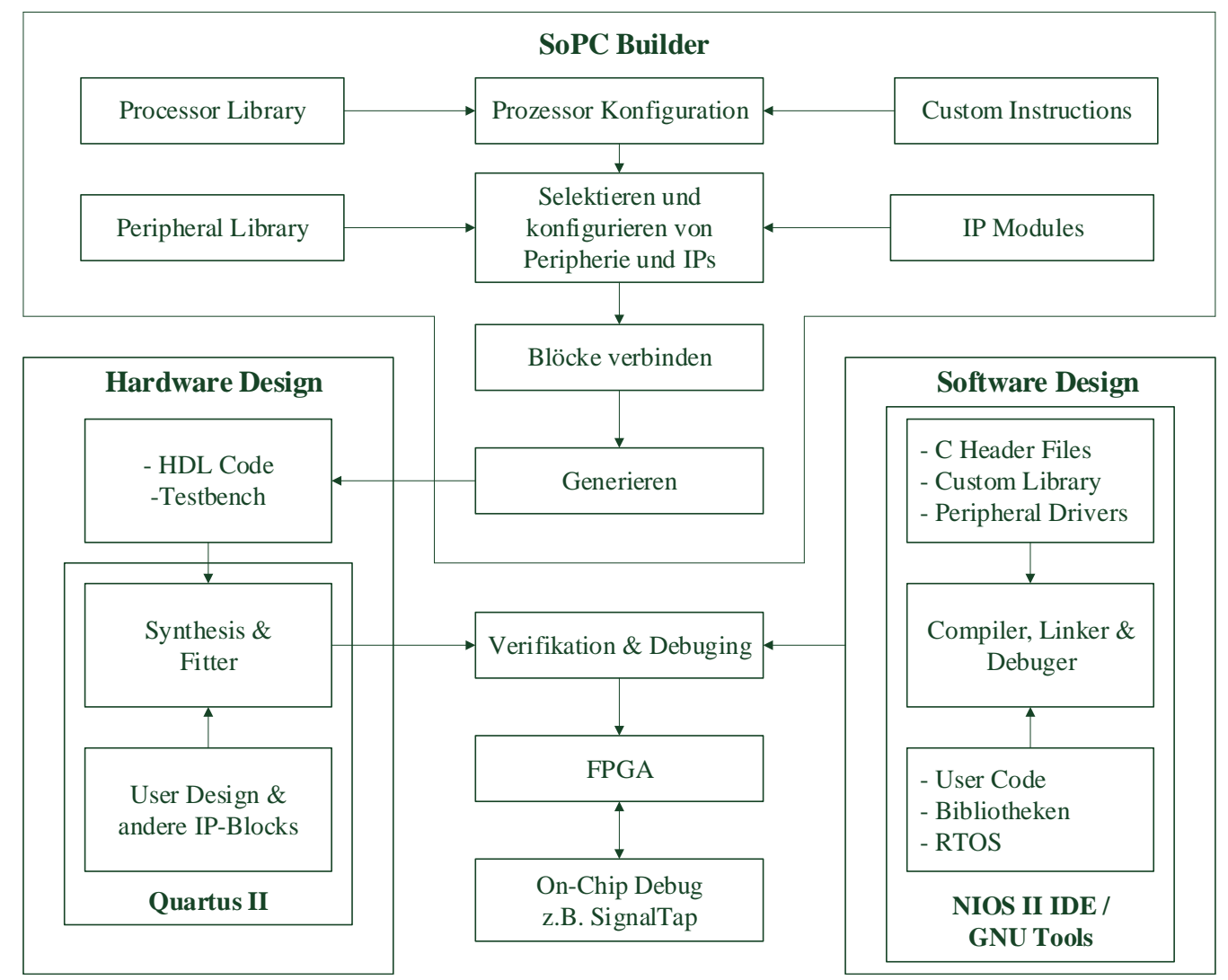

Abbildung 5.29.: Entwicklungsumgebung des SoPC-Systems [6].

bung für das Hardware-Design sowohl für FPGAs wie auch für CPLDs (engl.: Complex Programmable Logic Device). Die Software umfasst neben den Synthese-Funk- 
tionen und der Projektverwaltung auch ein Simulationstool. Neben dem internen Standard-Simulator besteht auch die Möglichkeit, die ModelSim-Altera Version [12] als zusätzliches Hardware-Simulations Werkzeug zu verwenden. SignalTap bezeichnet den leistungsfähigen internen Logic Analyzer. Der Logic Analyzer wird aus Logikelementen aufgebaut, die bei Testzwecken im FPGA synthetisiert werden. Der Logic Analyzer erlaubt, ähnlich einem Oszilloskop, die Signalverläufe im FPGA darzustellen. Dadurch ist eine Analyse und Verifizierung des generierten Codes möglich.

Ein weiteres Entwicklertool ist der SoPC-Builder. Das Entwicklertool ist das Basiswerkzeug zur Implementierung kompletter Mikroprozessorsysteme und IPs. Über die SoPC-Funktionalität kann der Soft-Core-Prozessor auf Basis vorhandener Komponenten konfiguriert und verwaltet werden. Über die ASF-Funktionalität werden die unterschiedlichen Funktionsblöcke des Design automatisch miteinander verbunden. Manuelle Verbindungen zwischen den IP-Blöcken oder die Verifizierung der Register bzw. Speicher entfallen, was den Entwicklungsprozess vereinfacht.

Die Nios II IDE (engl.: Integrated Design Environment) Entwicklungsumgebung stellt das Entwicklungswerkzeug für die komplette Software-Entwicklung des Nios II Prozessors zur Verfügung. Das Tool beinhaltet die Projektverwaltung, die CodeEntwicklung, einen Befehlsatz-Simulator und die Debugging Möglichkeit über die JTAG-basierte Schnittstelle (engl.: Joint Test Action Group). Als Betriebssystem wird das Echtzeit-Betriebssystem Micro/OS II verwendet.

Altera liefert mit dem Nios II ein parametriertes Modell, dessen Quellcode nicht offen gelegt ist. Zur leistungsabhängigen Skalierung für die Anwendung werden die drei Prozessorkerne: Economy, Standard und Fast zur Auswahl angeboten [8]. Über den SoPC-Builder kann der Entwickler eine Variante entsprechend der notwendigen Performance auswählen. Weiterhin lassen sich z.B noch die Anzahl der GP-Register oder die Größe des Instructions- und Datencaches einstellen. Alle drei Varianten unterstützen den gleichen Befehlssatz, d.h. es werden die gleichen Funktionen aufgerufen. Allerdings werden die Befehle unterschiedlich abgearbeitet. Benötigt z.B. die FastVersion beim Aufruf eines Hardware-Multiplizierer einen Taktzyklus, werden bei der Standard-Version schon drei Taktzyklen benötigt.

Bei der Economy-Version wird der Rechenalgorithmus in Software emuliert. Erkauft wird der Performance-Gewinn durch die Verwendung einer höheren Anzahl von Logikelementen. In dieser Arbeit wurde als Prozessorkern des Nios II die Fast-Variante gewählt. Die Variante zeigte sich bei den Versuchen als ausreichend leistungsfähig - auch hinsichtlich der benötigten logischen Elementen in der Arbeit (vgl. nachfolgende Beschreibungen) und die von der FPGA-Hardware zur Verfügung gestellten Ressourcen (vgl. Abschnitt 5.2.2). 


\section{SoPC-Designschritte}

Um das Design eines SoPC mit FPGA und integriertem Soft-Core zu erstellen, sind verschiedene Entwicklungsschritte notwendig. Abbildung 5.30 verdeutlicht die Design-Schritte für die Hardware- und Softwareentwicklung für die Umsetzung der FPGA-basierten Plattform. Das entwickelte Modell setzt sich zusammen aus dem gewählten konfigurierbaren Prozessorkern und die für die Peripherie zusätzlich notwendigen Bausteine. Der SoPC-Builder stellt für die Zusammenstellung der Bausteine eine Benutzeroberfläche zur Verfügung, die das Zielsystem graphisch beschreibt.

Nach der Wahl des für die Applikation geeigneten Prozessorkerns (Economy-, Standard- oder Fast-Variante) und den Peripherie-Bausteinen werden die gewählten Komponenten im SoPC-Builder synthetisiert bzw. durch VHDL- oder Verilog-Dateien exportiert. Vor der Synthetisierung werden der Prozessorkern und die eingebundenen Bausteine untereinander über die ASF-Busfunktionalität [9] miteinander verbunden. Die wesentlichen verwendeten Bausteine neben dem Prozessorkern in der Arbeit sind:

- interne Speicherkomponenten (Memory Interfaces wie RAM, ROM, SDRAM, Flash)

- JTAG Debug Modul (Auswahl der Breakpoints und der Data Trigger)

- externe Schnittstellen (vgl. Abbildung 5.7)

- Custom Instruction (vgl. nachfolgende Erläuterungen)

- IP-Bausteine für die Umsetzung der Regelung (vgl. Abbildung 5.33, eingezeichnete Bausteine im Feld „VHDL-Code“).

Um die Komponenten über den ASF-Bus miteinander zu verbinden, beinhaltet der ASF-Bus alle Kontroll- und Datenleitungen. Es besteht die Möglichkeit, beliebige simultane Verbindung zwischen den Blöcken zu generieren (Multi-Master-Multi-Slave Betrieb). Auch unterschiedliche Busbreiten können miteinander verbunden werden.

Dabei werden nicht alle Bausteine an den gemeinsamen Bus angeschlossen, sondern es werden nur die gewünschten Master-Slave Kombinationen mit dem SoPC-Builder ausgewählt, die für die Kommunikation der Bausteine untereinander benötigt werden. Der Avalon Bus definiert dabei nicht nur die Verbindungen der Datenleitungen und Kontrollleitungen sondern auch die notwendige Multiplexer- und Entscheiderlogik.

Durch die direkten Verbindungen der Blöcke untereinander wird, wie bereits angemerkt ein, Multi-Master-Multi-Slave Betrieb ermöglicht [4]. Erfolgt ein gleichzeitiger Zugriff auf einen gleichen Baustein von mehreren Mastern, regelt ein Entscheider auf der Slave Seite prioritätsabhängig den Zugriff. 

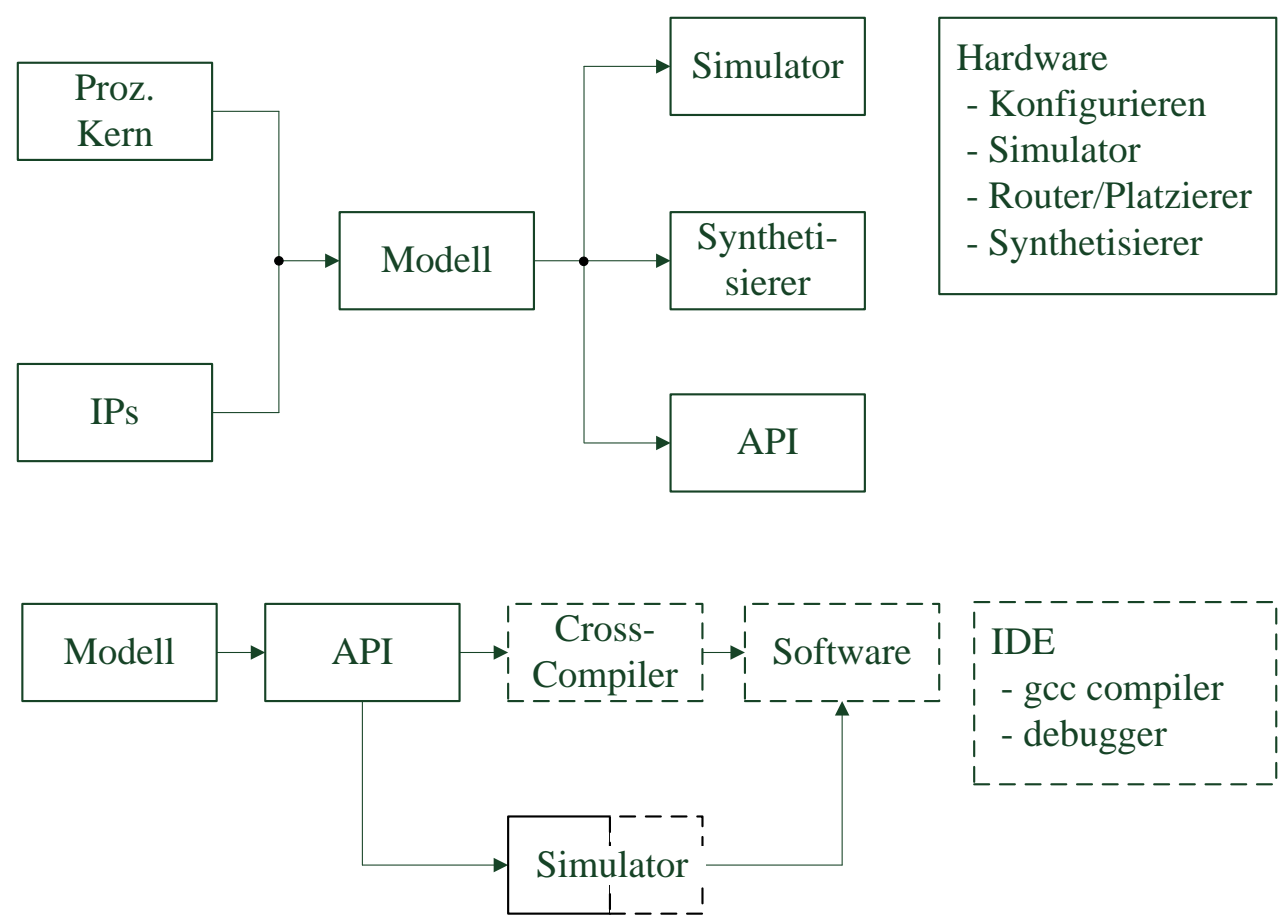

Abbildung 5.30.: Designschritte zum Aufbau des SoPC-Systems [6].

Mit dem Quartus II Simulator kann das erzeugte Modell auf mögliche Fehler untersucht werden. Nach der Synthetisierung wird die generierte Netzliste des Modells auf den FPGA geladen. Als weiterer Schritt wird eine API (engl.: Application Program Interface) erzeugt. Die Interface-Schnittstelle ermöglicht dem Softwareentwickler den Zugriff auf alle SoPC-Bausteine. Der Cross-Compiler erzeugt schließlich aus dem Modell bzw. aufbauend auf der API den systemspezifischen Code.

\section{Performance-Steigerungen}

Die Soft-Core-CPU Nios II bietet verschiedene Möglichkeiten, die Rechenleistung zu steigern bzw. die Performance an die jeweilige Anwendung anzupassen. Mittels sogenannter Custom Instructions (CI) [7] besteht die Möglichkeit, die ALUFunktionalität zu erweitern. Insgesamt stehen bis zu 256 anwenderspezifische Befehlssätze zur Verfügung. Zeitkritische Software-Algorithmen können durch Algorithmen in VHDL abgebildet werden, was den Softwarecode reduziert und die Berechnungsgeschwindigkeiten insgesamt erhöht. Umfangreiche oder komplexe Sequenzen von Standard-Instruktionen können dabei in einer einzigen Custom Instruction zusammengefasst bzw. implementiert werden.

Custom Instructions werden im SoPC-Builder eingebunden (Abbildung 5.29) und im 
Anwenderprogramm aufgerufen. In Abbildung 5.31 wird aus den beiden Eingängen im Register $A$ und Register $B$ direkt das Ergebnis im Register Result berechnet. Ein mehrmaliges Aufrufen der ALU ist nicht notwendig. Zu berücksichtigen ist, auch wenn die Abarbeitung in Hardware sehr schnell ist, dass das Anwenderprogramm nach dem Funktionsaufruf solange „wartet“, bis das Ergebnis von der ALU zurückgeliefert wird.

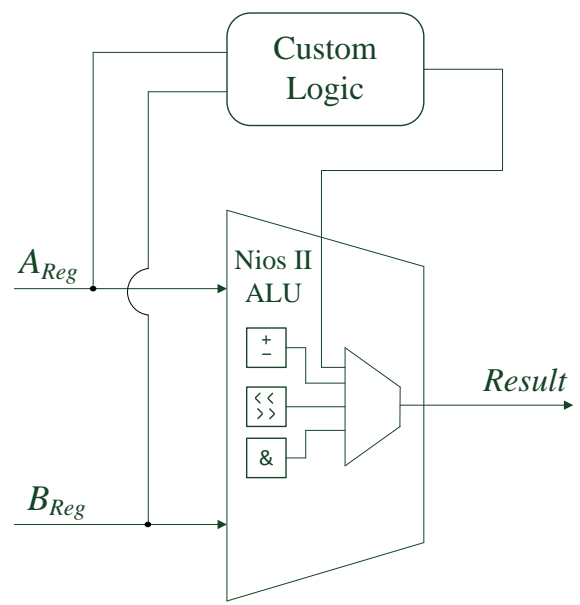

Abbildung 5.31.: Custom Instruction [7].

Eine weitere Möglichkeit, die Leistungsfähigkeit eines SoPC-Systems skalierbar zu steigern, ist die Einbindung von Co-Prozessoren und Multi-Prozessor-Systemen. Der Co-Prozessor wird oft so im SoPC-System integriert, dass er als Standard IP Block unabhängig von der Soft-Core-CPU eigene Logikfunktionen abbildet und die SoftCore-CPU so von rechenintensiven Aufgaben entlastet. Typische Anwendungsbeispiele sind FFT-Algorithmen oder Filter Berechnungen wie z.B. bei FIR (engl.: Finite Impulse Response) oder IIR (engl.: Infinite Impulse Response)-Filtern. Der ASF-Bus erlaubt durch die Simultaneous Multi-Mastering-Funktion einen parallelen zeitgleichen Zugriff auf die Peripherie, ohne dass das Bussystem zwischen Soft-Core-CPU und anderen kommunizierenden IP-Blöcken belastet wird.

Multi-Prozessor-Systeme ermöglichen darüber hinaus die Möglichkeit, Aufgaben auf mehrere Prozessorkerne aufzuteilen. Denkbar ist die Aufteilung in Bereiche, die steuerund regelungstechnische Aufgaben übernehmen oder Prozessoren, die die Ein- und Ausgabeverwaltung koordinieren - falls das gewünscht und sinnvoll ist. Als Ergebnis hinsichtlich der Umsetzung der notwendigen Soft-Core-Blöcke für die konkrete Applikation in der Arbeit konnte gezeigt werden, dass die Verwendung eines einfachen Prozessor-Systems, auch hinsichtlich einer skalierbaren und übersichtlichen Systemstruktur, ausreicht. 


\section{Motorsteuerungskonzept}

Für den Aufbau und die Realisierung der FPGA-basierten Motorsteuerung dient die im Abschnitt 5.2.1 beschriebene Referenzplattform als Leistungsteil und das im Abschnitt 5.2.2 beschriebene Development Board als Steuerplattform. Die Abbildung 5.32 zeigt den Versuchsaufbau der Anordnung. Die dargestellten Komponenten [4] bis [9] dienen als Testaufbau für die Untersuchung der Kommunikation über den induktiven Energieübertragungskanal (siehe Kapitel 4).

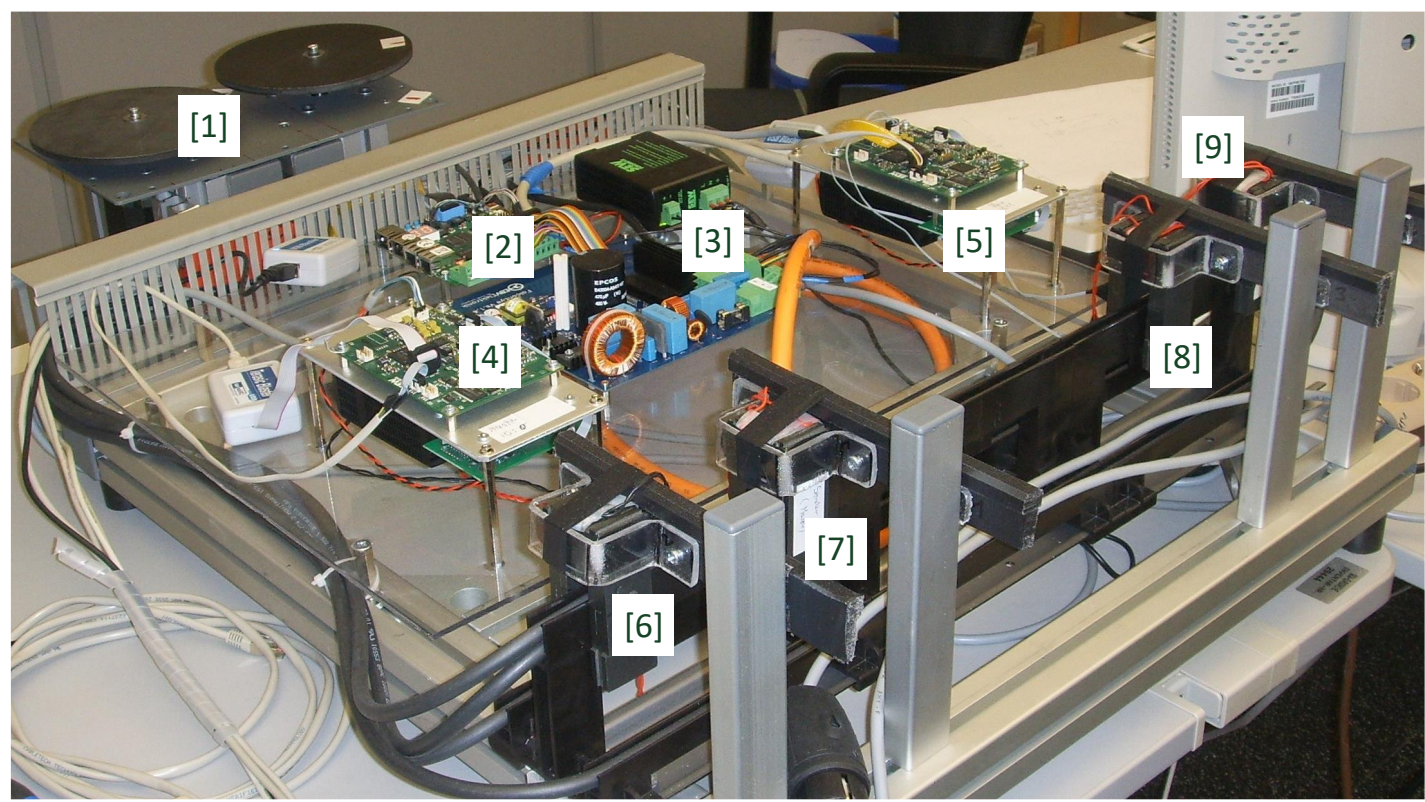

Abbildung 5.32.: Aufbau und Komponenten der Motorsteuerung. [1] Motor (PMSM), [2] Steuerteil des Umrichters, [3] Leistungsteil des Umrichters, [4] Modemplatine 1 Senden/Empfangen, [5] Modemplatine 2 Senden/Empfangen, [6] Sendeübertragerkopf Modemplatine 1, [7] Empfangsübertragerkopf Modemplatine 1, [8] Sendeübertragerkopf Modemplatine 2, [9] Empfangsübertragerkopf Modemplatine 2.

Die Leistungs- und Steuerplatine beinhalten alle elektronischen Bauteile, die für den Betrieb eines Drehstrommotors in FOC notwendig sind. Die Abbildung 5.33 zeigt übersichtlich die Schnittstellen zwischen beiden Hardware-Plattformen mit den einzelnen verwendeten Funktionsblöcken. Außer der Lagerückführung des Rotors sind alle Signale, die für die Kommunikation zwischen beiden Plattformen benötigt werden, binäre Signale. Die Signalpegel der Kopplungssignale betragen 3, $3 \mathrm{~V}$ und werden direkt vom FPGA ausgegeben bzw. eingelesen. Die sechs Steuersignale für die IGBTs sind auf der Leistungsplatine über Optokoppler galvanisch getrennt.

Das zentrale Bauteil auf der Steuerplatine bildet der EP3C40-FPGA aus der Cyclone- 


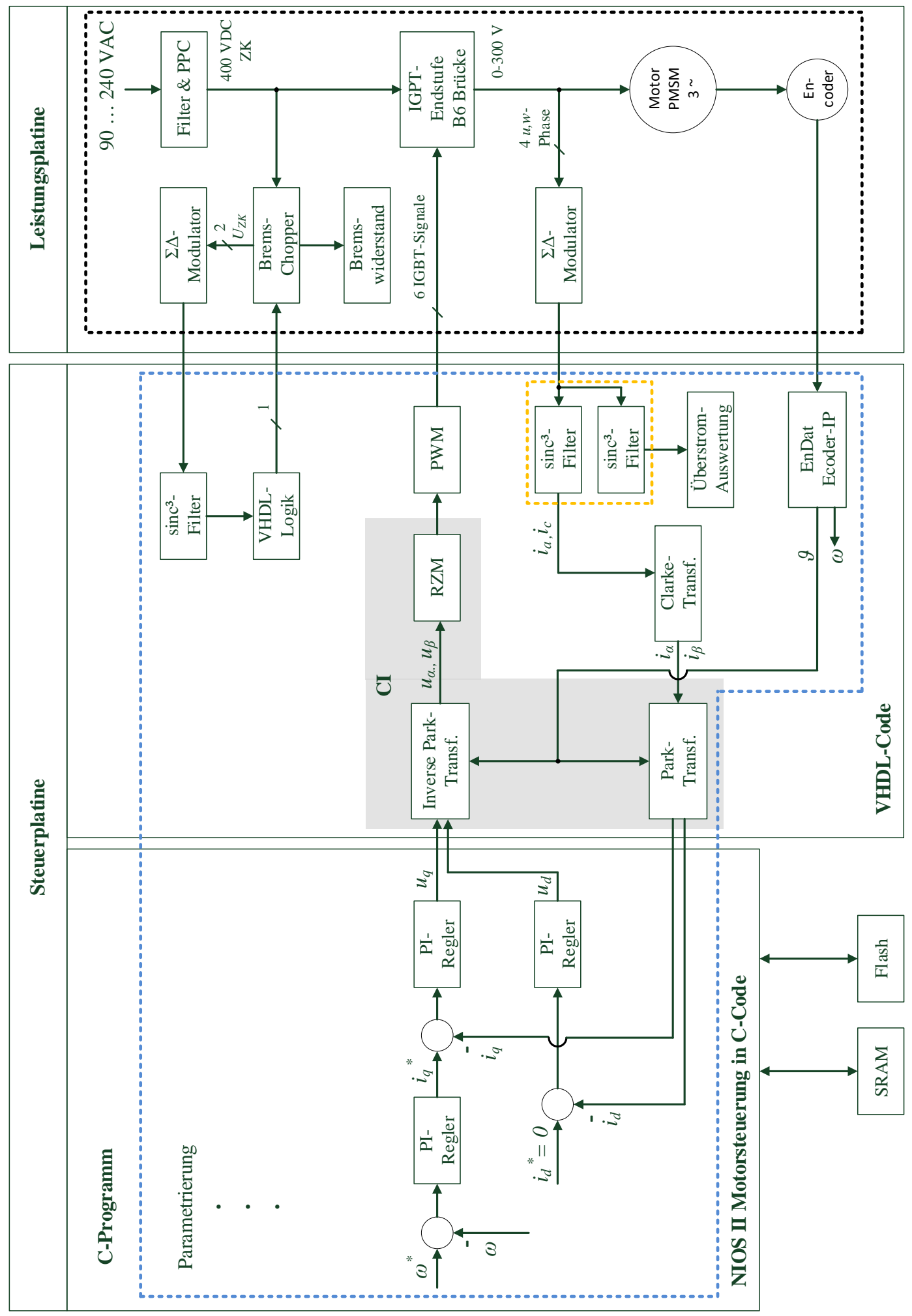

Abbildung 5.33.: Blockschaltbild der Motorsteuerung. 
III Familie der Fa. Altera. Mit solchen leistungsfähigen FPGAs ist die Realisierung der Steuerung für einen Servoantrieb mit einem Chip realisierbar. Der Aufbau innerhalb des FPGAs zeichnet sich aus durch die Integration mehrerer IP-Blöcke für verschiedene Funktionen und Aufgaben, die über den ASF-Bus mit dem konfigurierbaren Soft-Core-Mikrocontroller Nios II verbunden sind.

Der ASF-Bus ermöglicht Multi-Master-Multi-Slave-Betrieb mit Simultaneous MultiMastering Funktion (siehe Abschnitt 5.3.5). Der Soft-Core-Kern wird dabei mit $100 \mathrm{MHz}$ getaktet - die Peripherie, Flash und das externe RAM mit einer Frequenz von $50 \mathrm{MHz}$. Bei der schnellsten Variante der Soft-Core-CPU, die auch im Versuchsaufbau verwendet wurde, beträgt die Rechenleistung ca. 1,1 MIPS (engl.: Million Instructions Per Second) pro $\mathrm{MHz}$ und ist somit vergleichbar mit klassischen Mikrocontrollern [5], [109]. Dabei haben Befehls- und Daten-Cache Zugriff auf den schnellen On-Chip-Speicher.

Die Vorteile eines FPGAs in Verbindung mit der Soft-Core-CPU gegenüber einem klassischen Mikrocontroller sind einerseits die Möglichkeit, die Konfiguration der Peripherie selbst anzupassen, aber auch auf der anderen Seite, flexibel auf Veränderungen der Hardwareanforderungen zu reagieren. Der Befehlssatz kann durch benutzerdefinierte Anweisungen (CI, engl.: Custom Instruction) universell angepasst werden. Rechenzeitintensive Operationen können so vollständig in VHDL bzw. in Hardware abgebildet werden. Zusätzliche funktionelle Erweiterungen sind möglich, die ein klassischer Mikrocontroller nicht bieten kann. Auch ergeben sich weniger Abhängigkeiten hinsichtlich des gegebenen Funktionsumfanges des Mikroprozessor-Herstellers.

Durch die schnellen Ausführungen der rechenintensiven Berechnungen in Hardware werden Anfragen für Rechenergebnisse während des Programmdurchlaufes direkt abgesetzt und unmittelbar darauf die Ergebnisse wieder abgefragt. Dadurch sind im Vergleich zu einem klassischen Mikrocontroller auch weniger Interrupt-Eingriffe während des Programmdurchlaufes notwendig. Aufwändige Registersicherungen, wie z.B. von Stapel- und Programmzähler, müssen nicht durchgeführt werden - es ergibt sich eine übersichtliche Programmstruktur.

Erkauft werden die genannten Vorteile des FPGAs durch einen immer noch aktuell höheren Einzelstückpreis auch bei größeren Abnahmemengen im Vergleich zum angepassten Mikrocontroller und durch die Notwendigkeit, dass bei der Softwareentwicklung neben der gebräuchlichen Beherrschung des C-Codes auch Kenntnisse und Kompetenzen seitens der VHDL-Synthetisierung gegeben sein müssen.

Die durchgeführten Untersuchungen am Versuchsaufbau (vgl. Abbildung 5.32) zeigen, dass es unter den aufgeführten Bedingungen durch die Verwendung des FPGAs im Bereich der Motion-Control Anwendung möglich ist, den Programmdurchlauf für die Berechnung eines Drehzahlregelkreises mit unterlagerter Stromregelung eines Drehstrommotors (BLCD) in der feldorientierten Regelung dynamisch in ca. $5 \mu s \mathrm{zu}$ 
realisieren. Die Bearbeitungszeiten wurden durch parallele Messungen mit dem internen Logic Analyzer bzw. Debugging Programm SignalTap und einem Oszilloskop bestimmt.

Bei klassischen Mikrocontrollern orientieren sich die Bearbeitungszeiten bzw. die Taktzeiten sehr stark an den konkreten Anforderungen des Anwendungsbereiches. Am Markt werden dafür Mikroprozessoren mit Taktraten ab ca. 40 MHZ bis in den dreistelligen Megahertz-Bereich angeboten [211], [215]. Dabei sind solche Prozessoren in der Regel mit hinterlegten Funktionen für die Ansteuerung der Pulsweitenmodulation (PWM) und zusätzlichen integrierten Leistungsmerkmalen, auch zur Anbindung an die Peripherie, ausgestattet (vgl. Abschnitt 5.1).

Für Motion-Control Anwendungen im mittleren Leistungsbereich zwischen ca. $1 \mathrm{~kW}$ ... $100 \mathrm{~kW}$ sind PWM-Frequenzen von $4 \mathrm{kHz}, 8 \mathrm{kHz}$ und $16 \mathrm{kHz}$ üblich. Bei einer PWM-Frequenz von z.B. $16 \mathrm{kHz}$ beträgt die Stromregelzeit entsprechend 62,5 $\mu \mathrm{s}$. Für kleinere Leistungen unter $1 \mathrm{~kW}$ kommen immer mehr schnellschaltende MOSFETS (engl.: Metal Oxide Semiconductor Field-Effect Transistor) mit hohen Sperrspannungen zum Einsatz, die mit PWM-Frequenzen von weit über $20 \mathrm{kHz}$ betrieben werden.

Für die in Abbildung 5.33 dargestellte Konfiguration sind für die Umsetzung der FOC ca. 6000 Logikelemente im FPGA notwendig (vgl. Abschnitt 5.2.2). Die beiden PIRegler für die Stromregelung des $d$ - und $q$-Anteiles sowie der Drehzahlregler sind in der Umsetzung des Regelungskonzeptes in der Programmiersprache $C$ programmiert (linker Bildbereich in Abbildung 5.33 „NIOS II Motorsteuerung in C-Code“). Nach der Compilierung werden die Programmteile durch die Soft-Core-CPU abgearbeitet. Die rechenintensiven Bestimmungen der Stellgrößenbegrenzungen für die PI-Regler werden im C-Programm durch CI-Aufrufe unterstützt.

Für die Umsetzung der Funktionsblöcke auf der SoPC-basierten Systemplattform mit Soft-Core-CPU besteht generell die Möglichkeit, die Blöcke in C-Code, als CI oder als IP-Block in VHDL umzusetzen. Allerdings ergibt sich aus den Anforderungen hinsichtlich der zulässigen Bearbeitungszeiten und den benötigten Rechenleistungen von bestimmten Funktionsblöcken eine bindende Zuordnung. Besteht eine Alternative, die Funktionsblöcke in C-Code oder in VHDL bzw. als CI zu realisieren, ist ein Kompromiss zwischen benötigter Prozessor-Bearbeitungszeit und notwendigen Logikelementen zu finden. Die Höhe der verfügbaren Logikelemente wird dabei durch den gewählten FPGA-Typ festgelegt. Die Synthetisierung in VHDL ist dabei sehr schnell, aber weniger flexibel wie im C-Programm. Durch die Einbindung von CIs werden übersichtliche und flexible Systemstrukturen erreicht.

Die zeit- und rechenintensiven Algorithmen, wie die PWM für die Ansteuerung der 6 IGBTs, die Logik und Auswertung für die Bestimmung der Motorströme und der Bereich für die ZK-Regelung sind im realisierten Versuchsaufbau als eigenständige 
IP-Blöcke hinsichtlich der Anforderungen der FOR eingebunden. Auch die ClarkeTransformation wurde als IP-Modul umgesetzt (mittlerer Bereich in Abbildung 5.33 „VHDL-Code“). Für die Rückführung der Lageinformation des Rotors ist auf dem Motor eine schnelle EnDat-Encoder-Schnittstelle [71] verfügbar. Die Werte der digitalen hochaufösenden Lageinformation werden durch einen EnDat VHDL-Block ausgewertet und über den Avalon-Bus abgefragt.

Hier bietet der FPGA durch seine dynamische Taktzeit von 20 ns ideale Einsatzgebiete. Modulerweiterungen sind ohne Probleme möglich. Spezielle periphere Modulblöcke wie z.B. für die PWM-Ansteuerung, wie sie in klassischen Mikrocontrollern vorkommen bzw. notwendig sind, werden nicht benötigt. Durch die saubere Trennung der Funktionsabläufe und der exakten Definition der Schnittstellen zwischen den einzelnen Modulen zeigt sich eine solche Herangehensweise betreffend der Aufteilung in unterschiedlichen Funktionsgruppen als sinnvoll und praktikabel. Die IP-Blöcke für die PWM und der EnDat-IP Block wurden dabei in der Umsetzung als fertige Bausteine übernommen (vgl. [49]) und hinsichtlich der Schnittstellen an die bestehende Struktur angepasst.

VHDL-Module können generell als IP-Blöcke oder als CIs in die Regelungsstruktur eingebunden werden. Im Konzept des kompakten Aktors ist die Raumzeigermodulation, welche aus dem Spannungsraumzeiger in Ständerkoordinaten die Eingangswerte bzw. die PWM-Schwellwerte berechnet, als CI realisiert. Gleiches gilt für die Park-Hin- und Rücktransformation. Die Vorgehensweise hat dann Vorteile, wenn z.B. durch die Regelungsplattform zusätzliche Antriebe angesteuert werden. In einem solchen Anwendungsfall sind weniger Logikelemente im FPGA notwendig, da die Modulblöcke nur einmal instanziiert werden müssen. Durch die Instanziierung ist es innerhalb der Softwareumgebung möglich, die Bausteine mehrfach zu nutzen bzw. aufzurufen. Der Ansatz und die Struktur entspricht dem Aufbau eines kompakten modularen Aktors, der für verschiedene Aufgaben und Anwendungen Verwendung findet. Solche Strukturen wurden auch in [109] betrachtet und vorgestellt.

Für die Strommessung der Motorphasen $u$ und $w$ und für die Bestimmung der Zwischenkreisspannung sind auf der Leistungsplatine drei $\Sigma \Delta$-Stromwandler integriert, die den hochfrequenten Bitstrom bzw. Bitstream zum FPGA übertragen (vgl. Abschnitt 5.3.5). Der Bitstream wird vom FPGA direkt eingelesen und verarbeitet. Dabei wird der Bitstream zwischen Leistungsteil und Steuerplatine auf der Leistungsplatine einfach mittels Optokoppler galvanisch getrennt, was einen weiteren wichtigen Vorteil der $\Sigma \Delta$-Technologie darstellt. Bei industriellen Umrichtern sind getrennte Potentialverbindungen oftmals erwünscht oder eine Forderung - auch hinsichtlich der Störsicherheit und Zuverlässigkeit.

Wird im Fall der Zwischenkreisspannung des Umrichters der Wert der zulässigen maximalen Spannung überschritten, wird vom FPGA-Board ein Gate-Treiber auf der 
Leistungsplatine angesteuert (vgl. Abschnitt 5.2.1). Der Gate-Treiber-Baustein steuert wiederum einen Transistor an (Bremschopper in Abbildung 5.33), der bei einer zu hohen Zwischenkreisspannung einen Bremswiderstand zuschaltet (Bremschopper in Abbildung 5.33). Die überschüssige Energie im Zwischenkreis wird dadurch in Wärme umgewandelt. Durch die im FPGA realisierte schnelle hardwareseitige Überwachungsschaltung in Kombination mit der schnellen Strommessung im Zwischenkreis können die Schaltgrenzen für die Zwischenkreis-Spannungsregelung einfach eingestellt und überwacht werden. Eine Überladung des Zwischenkreiskondensators, die zwangsweise zur Zerstörung des Bauteils führt, wird verhindert.

Im konkreten Aufbau für die Strommessung der Motorsteuerung stehen $\Sigma \Delta$-Modulatoren 2. Ordnung mit einer Abtastfrequenz von $10 \mathrm{M} \mathrm{Hz}$ auf der Platine des Leistungsteils zur Verfügung (siehe Abschnitt 5.2.1). Der einfache und robuste Aufbau auf der Hardwareseite mit nur wenigen benötigten analogen Bauteilen erfordert auf der Auswerteseite allerdings eine aufwendigere digitale Filterung. Dabei hat die Filterung die Aufgabe, den hochfrequenten Rauschanteil im Bitstream-Signal zu unterdrücken. Im FPGA sind solche Filter als sinc ${ }^{k}$-Filter, wie in Abschnitt 5.3.5 bereits beschrieben, in VHDL einfach synthetisierbar [235].

Der Faktor $k$ steht dabei für die Ordnung des Filters. Das überabgetastete Messsignal wird zuerst $k$-mal integriert, dann mit einer einstellbaren niedrigeren Frequenz abgetastet und im letzten Schritt $k$-mal differenziert. Damit sich der Signal-RauschAbstand im Digitalteil nicht verschlechtert, wird typischerweise die Ordnung des Filters eine Ordnungsstufe höher gewählt als die Ordnung des Modulators. Bei dem hier verwendeten Modulator 2. Ordnung wurde daher zweckmäßigerweise ein Filter 3. Ordnung realisiert und integriert.

Tabelle 5.3 zeigt die Abhängigkeiten der Bandbreite $f_{B}$ und die regelungstechnisch wirksame Verzögerungszeit $T_{\Sigma}$ in Abhängigkeit vom Oversampling-Faktor $M$ für einen Filter 3. Ordnung und eine Modulator-Taktfrequenz von $f_{\Sigma \Delta}=10 \mathrm{MHz}$ [235]. Die Werte in der Tabelle 5.3 berechnen sich gemäß Gl. (5.57) und Gl. (5.58).

Ein hoher Oversampling-Faktor $M$ verbessert die Rauschunterdrückung (vgl. Abbildung 5.24) und die Genauigkeit bzw. die Auflösung des Signals. Bei niedriger Eckfrequenz steigt aber auch die notwendige Auswertezeit und damit die effektive Totzeit des Systems.

Für jede Phasenstrommessung sind im FPGA zwei $\operatorname{sinc}{ }^{3}$-Filter mit unterschiedlichen Filterzeitkonstanten realisiert. Durch die Parallelverarbeitung im VHDL-Block ist es möglich, zwei Signalpfade gleichzeitig auszuwerten (vgl. Abbildung 5.33, gelb schraffierter Bereich). Eine geringere Genauigkeit, $M=8 \ldots 16$, bei kurzen Reaktionszeiten ist ausreichend für eine schnelle Überstrom-Überwachung der Leistungshalbleiter. Für die Stromreglung der FOC wird eine höhere Genauigkeit, $M=64 \ldots 128$, 
bei längerer Bearbeitungszeit benötigt. Klassische analoge Komparatoren zur Überstromerkennung und analoge Filter zur Störunterdrückung sind für die Strommessung im FPGA nicht mehr notwendig.

Tabelle 5.3.: Werte des $\operatorname{sinc}^{3}$-Filters in Abhängigkeit von $M$ und $f_{\Sigma \Delta}$ [146], [235].

\begin{tabular}{lll}
\hline $\mathrm{M}$ & Bandbreite $f_{B}[k H z]$ & Verzögerungszeit $T_{\Sigma}[\mu s]$ \\
\hline 16 & 164 & 2,4 \\
\hline 64 & 41 & 9,6 \\
\hline 128 & 21 & 19,2 \\
\hline
\end{tabular}

$$
\begin{aligned}
\text { mit } f_{B} & =\frac{f_{\Sigma \Delta}}{3,8 M} \text { und } \\
T_{\Sigma} & =\frac{1,5 M}{f_{\Sigma \Delta}} .
\end{aligned}
$$

In der Abbildung 5.34 ist beispielhaft der Betrieb der Motorsteuerung auf Basis einer FPGA-basierten Positionsregelung für die PMSM im Leerlauf der Maschine abgebildet. Im obersten Bild in Abbildung 5.34 wird die Sollposition über eine Sollwerttrajektorie vorgegeben. Insgesamt dreht der Antrieb 30 Umdrehungen und erreicht dabei kurzzeitig eine Geschwindigkeit von $600 \mathrm{U} / \mathrm{min}$ (mittleres Bild). Das untere Bild zeigt die Istposition der Drehbewegung.

Die aktuellen Soll- und Istwerte der Regelung wurden beim Betrieb des Motors im internen Register des FPGA aufgezeichnet und über die serielle Schnittstelle des FPGA-Boards anschließend ausgelesen und aufgezeichnet. Für die Einstellung der Regelparameter des Strom- und Drehzahlregelkreises wurden die in Abschnitt 5.3.3 beschriebenen Grundeinstellwerte verwendet und anschließend im Probebetrieb optimiert. Der Antrieb zeigt einen zufriedenstellenden und stabilen Verlauf. 5.3.3

\subsection{Zusammenfassung der Ergebnisse}

Mit einer SoPC-basierten Systemplattform wurde eine variable und anpassbare elektronische Systemstruktur für eine Motorsteuerung vorgestellt und aufgebaut. Sie bietet eine Alternative zu einem klassischen Mikrocontrollersystem. 

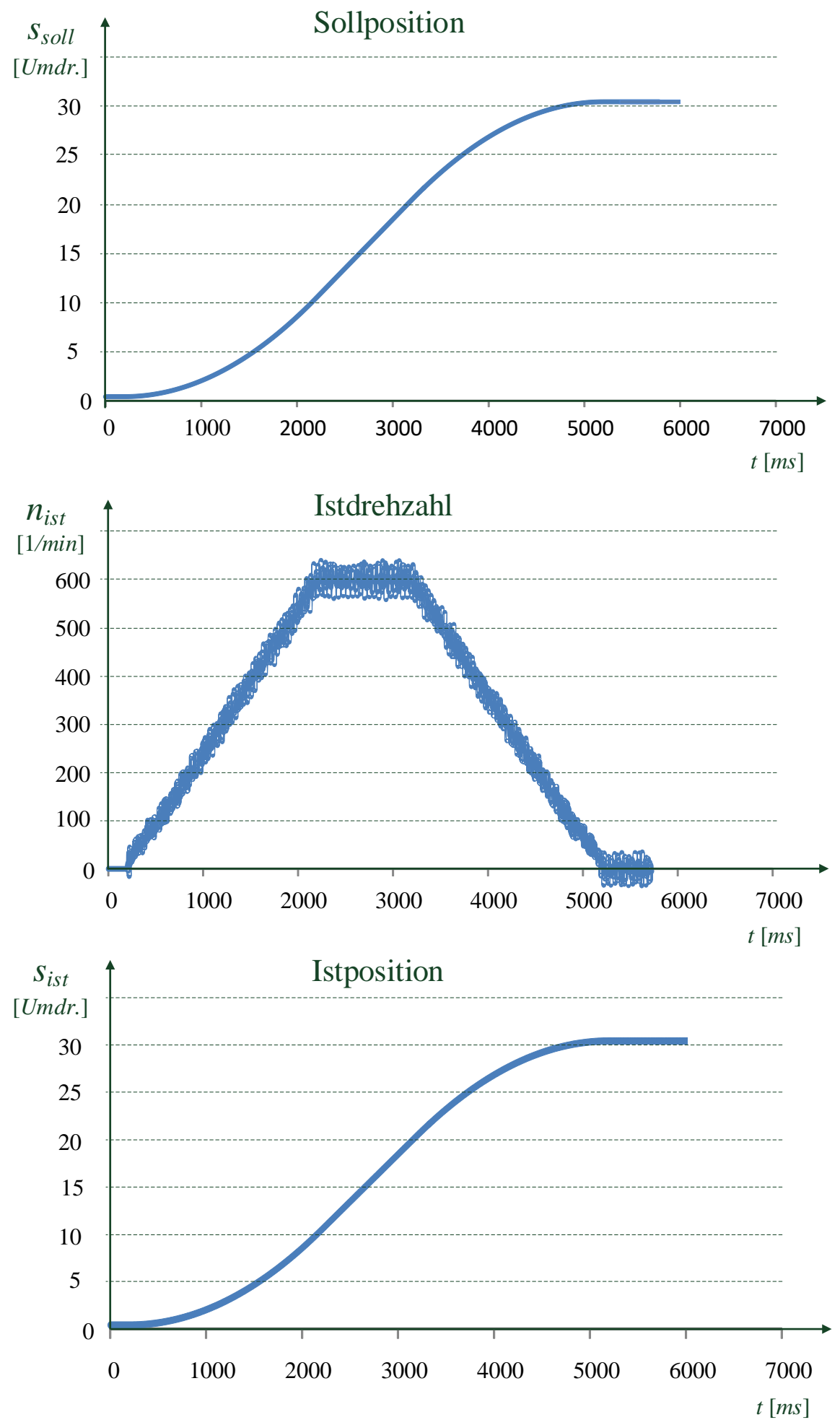

Abbildung 5.34.: FPGA-basierte Positionsregelung. 
Zu Beginn von Kapitel 5 werden die Eigenschaften und die Unterschiede von Mikrocontrollern und FPGAs aufgezeigt. Die Bauteile und Komponenten der Steuerplatine und der dezentralen Leistungselektronik sowie die Schnittstelle zwischen beiden Baugruppen wurden beschrieben. Der Aufbau und die Wirkungsweise der PMSM, die mathematische Zusammenhänge für die Umsetzung der FOC und die Realisierung der Strom- und Drehzahlregelung wurden erläutert und dargestellt. Die Grundlagen für die Auslegungen und Einstellungen des Drehzahlreglers und der Stromregler im $d$ - und $q$-Zweig der Stromregelung wurden erarbeitet.

In der Realisierung der Motorsteuerung sind der Drehzahlregler und die Stromregler programmtechnisch im Bereich der Soft-Core-CPU in der Programmiersprache $C$ umgesetzt. Bei der Umsetzung der Regelungs- und Funktionsblöcke zeigte sich, dass sich die Online-Berechnungen für die Koordinatentransformationen wie ParkTransformation, Inverse Park-Transformation sowie Clarke-Transformation effizient in VHDL-Code bzw. als Custom Instruction einbinden lassen. Auch die Umsetzung der Raumzeiger- und Pulsweitenmodulation sowie die Ansteuerung des BremsChoppers sind in VHDL-Code für Echtzeitanforderungen synthetisierbar.

Durch die Verwendung von modernen $\Sigma \Delta$-Modulatoren und einem angepassten digitalen Dezimierungsfilter lässt sich die wichtige Phasenstrommessung von Drehstrommotoren in Kombination mit FPGA-basierter Technik gegenüber klassischen Strommessverfahren deutlich vereinfachen und verbessern. Darüber hinaus ist es möglich, die Phasenstrommessung von Drehstrommotoren für unterschiedliche Genauigkeitsanforderungen und Reaktionszeiten einfach zu bestimmen.

Weiter konnte gezeigt werden, dass es durch die Verwendung FPGA-basierter Technik möglich ist, eine sehr kompakte modulare Motorsteuerung innerhalb des Konzeptes eines intelligenten Aktors im Bereich der beweglichen Verbraucher aufzubauen. Die Einbindung zusätzlicher Soft- und IP-Cores ermöglicht innerhalb des SoPC-basierten Embedded Systems den Aufbau einer skalierbaren Systemplattform, dabei können zusätzliche Aktoren oder andere ergänzende Eigenschaften und Funktionen mit eingebunden werden.

Der im Blockschaltbild der Motorsteuerung (Abbildung 5.33) abgebildete blau schraffierte Bereich der Hardware- und Softwareplattform des intelligenten Aktor entspricht dabei dem blau schraffierten Bereich im vereinfachten vorgestellten Konzept in Kapitel 2, Abbildung 2.2. Die Ansteuerung der dezentralen Leistungselektronik für die PMSM im Blockschaltbild in Abbildung 5.33 (schwarz schraffierte Bereich) entspricht dem schwarzen Bereich im Gesamtkonzept in der in Abbildung 2.2.

Im nächsten Kapitel wird untersucht, welche alternativen Regelungsverfahren es zu der in Kapitel 5 realisierten PI-Regelung noch gibt und wie sie in das Gesamtkonzept eines kompakten intelligenten Aktor eingebunden werden können. 


\section{Entwicklung eines Regelungskonzeptes mit prädiktiven Verhalten}

In diesem Kapitel wird in einer Simulation gezeigt, inwieweit modellprädiktive Regelverfahren (MPC, engl.: Model Predictive Control) im Konzept für bewegliche elektrische Verbraucher bzw. intelligente Aktoren eingebunden werden können. Die Überlegung geht davon aus, dass durch die heute zur Verfügung stehende Rechenleistung, gerade im Bereich der FPGA-Anwendungen, rechenintensive Regelverfahren wie die MPC-Regelung [27], [155] zukünftig auch immer stärker wirtschaftlich umgesetzt werden können. Untersuchungen zur Integration solcher Regelverfahren im Bereich elektrischer Antriebe wurde z.B. in [27], [122] betrachtet.

Im ersten Abschnitt von Kapitel 6 wird auf die grundlegende Funktionsweise von modellprädiktiven Regelverfahren eingegangen. Im weiteren Verlauf werden zwei der bekanntesten modellprädiktiven Basis-Regelverfahren für lineare Prozessmodelle vorgestellt. Das Dynamic Matrix Control Verfahren verwendet ein Gewichtsfolgemodell, das Generalized Predictive Control Verfahren ein zeitdiskretes ÜbertragungsfunktionsModell für die Vorhersage.

In Abschnitt 6.2 wird detailliert die Modellbildung der PMSM im statorfesten und rotorfesten Koordinatensystem beschrieben. Das Modell der Synchronmaschine bildet den Ausgangspunkt für die durchgeführten Simulationen mit dem linearisierten Modell der PMSM im Zustandsraum, welches für den Reglerentwurf des MPC-Reglers benötigt wird.

Anschließend wird dann in Abschnitt 6.3.4 ein Vergleich der Leistungsfähigkeit der MPC-Regelung zu einer klassischen PID-Regelung in einer Simulation dargestellt. In der Simulation wird die Regelung der PMSM hinsichtlich Führungs- und Störverhalten untersucht.

Die vorgestellte Untersuchung in Kapitel 6 legt keinen Anspruch auf Vollständigkeit hinsichtlich der Einsatzmöglichkeit modellprädiktiver Regelungen. Vielmehr soll gezeigt werden, wie MPC-Regelungen im industriellen Umfeld auch für elektrisch dynamische Antriebe eingesetzt werden können. Zunächst wird im nachfolgenden 
Abschnitt die grundlegende Funktionsweise von modellprädiktiven Regelverfahren beschrieben.

\subsection{Darstellung modellprädiktiver Regelverfahren}

Modellprädiktive Regelverfahren, auch als modellbasierte prädiktive Regelungen bezeichnet, sind Regelungsalgorithmen, die mit Hilfe eines internen Prozessmodells zukünftige Regeldifferenzen abschätzen und daraus einen optimalen Stellgrößenverlauf berechnen. Dabei werden Regelziele durch die Definition einer Gütefunktion festgelegt. Anders als bei klassischen Regelungsverfahren wird das Modell, welches das dynamische Verhalten des Prozesses beschreibt, nicht nur in der Entwurfsphase herangezogen, sondern es findet auch Verwendung im laufenden Betrieb der Regelung. Eine Einführung und eine Übersicht zum Thema findet sich in [45], weitere Literatur in [23], [27], [110], [113], [122].

Erste Ideen für MPC-Regelungen wurden bereits in den 60er-Jahren formuliert [36]. In den 70er-Jahren fanden erste prädiktive Regelungsverfahren Verwendung in der petrochemischen Industrie. Zur Regelung der langsamen chemischen und thermischen Prozesse waren geringere Abtastraten ausreichend und ermöglichten so den Einsatz der relativ rechenaufwändigen prädiktiven Regelung.

Nach dem erfolgreichen Einsatz in der Industrie wurde das Thema der prädiktiven Regelung Mitte der 80er-Jahre auch im universitären Bereich aufgegriffen. Das Regelungsverfahren hat sich mittlerweile in den verfahrenstechnischen Bereichen vor allem auch deshalb durchgesetzt, da hier viele Prozesse einen Mehrgrößencharakter besitzen. Mit MPC-Algorithmen können solche Prozesse effektiv geregelt werden. Abhängigkeiten in den Prozessen, in denen sich gleichzeitig mehrere Regelgrößen wie z.B. Temperatur, Druck, Volumenstände gegenseitig beeinflussen, sind mit klassischen Regelkonzepten schwer abbildbar. Darüber hinaus ist es der MPC-Regelung möglich, Nebenbedingungen für die Stell- und Regelgrößen im Regelungsalgorithmus zu berücksichtigen.

Oftmals fungiert die MPC-Regelung in den verfahrenstechnischen Prozessen als übergeordnete Basisregelung, welche die stark verkoppelten sich langsam ändernden Prozessgrößen regelt bzw. koordiniert und die dann daraus ermittelten Sollwerte der einzeln zu regelnden Prozessgrößen an unterlagerte einschleifige PID-Regelungen übergibt [168]. Die einzelnen PID-Kreise können dann unabhängig voneinander entworfen und eingestellt werden. Heute gelten MPC-Regelungen in der verfahrenstechnischen Industrie als de-facto-Standard für high-performance-Regelungen [167]. Ergänzende Informationen zur industriellen Anwendung von modellprädiktiven Regelungen finden sich in [67], [162], [163]. 
Durch die Verwendung von leistungsfähigen Mikroprozessoren und FPGAs und die dadurch hohe verfügbare Rechenleistung treten auch Anwendungen im Bereich der elektrischen Antriebe und anderen schnellen Applikationen stärker in den Fokus der Forschungsgemeinschaft.

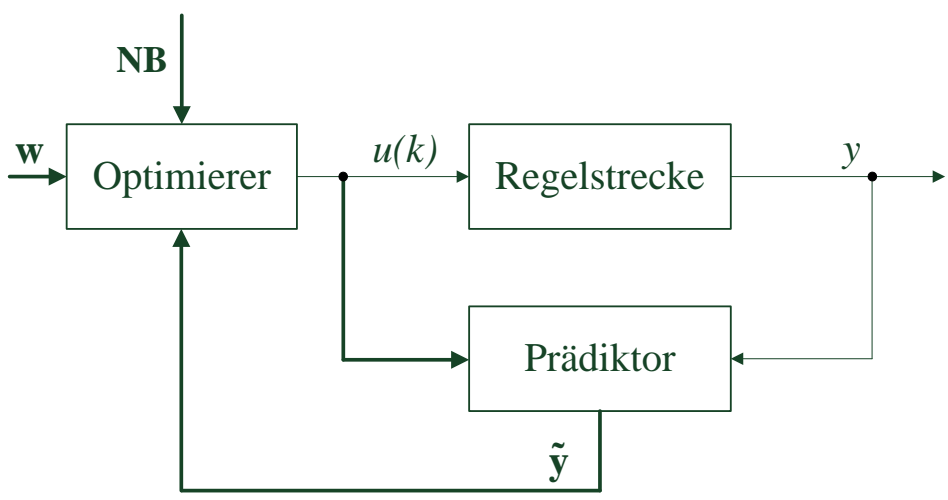

Abbildung 6.1.: Struktur einer prädiktiven Regelung [167].

Abbildung 6.1 zeigt die prinzipielle Struktur einer prädiktiven Regelung. Ein Optimierer und ein Prädiktor bilden die wesentlichen Bestandteile des MPC-Verfahrens. Die Aufgabe des Prädiktors ist es, auf der Grundlage eines geeigneten Prozessmodells, das zukünftige Prozessverhalten vorherzusagen. Ausgangsgröße des Prädiktors ist das prädizierte Ausgangssignal $\tilde{\mathbf{y}}$, das vom Prädiktor geschätzt wird. Bei der Schätzung werden auch zukünftige Stellgrößen $u(k) \ldots u\left(k+N_{u}\right)$ und der aktuelle Prozesszustand $y(k)$ mit einbezogen. Durch die Rückführung des prädizierten Ausgangssignals $\tilde{\mathbf{y}}$ wird der Regelkreis quasi geschlossen. Eine direkte Rückkopplung wie in klassischen Regelungssystemen besteht allerdings nicht. Der Sachverhalt stellt ein wesentliches Erkennungsmerkmal der prädiktiven Regelung dar.

Der Optimierer berechnet aus dem konstanten Sollwert $w$ bzw. wenn vorhanden aus dem Verlauf der hinterlegten zukünftigen Sollwerttrajektorie w, den Nebenbedingungen (NB) und des prädizierten Ausgangssignals $\tilde{\mathbf{y}}$ die optimale Stellgrößenfolge. Allerdings wird nur das erste Element der Stellgrößenfolge, $u(k)$, am Ausgang des Optimierers als Stellgrößenvorgabe auf die Regelstrecke geschaltet.

Abbildung 6.2 zeigt die prinzipielle Funktionsweise der MPC-Regelung. Wichtige beschreibende Größen der Regelung sind Steuerhorizont und Prädiktionshorizont. Die Beschreibung bezieht sich auf eine prädiktive Regelung eines linearen Prozessmodells und eines Single-Input Single-Output Systems (SISO), d.h. wie in der Abbildung 6.2 ersichtlich, nur mit einer Regelgröße und einer Steuergröße. Eine Darstellung für Mehrgrößensysteme findet sich in [23]. Literatur zum Thema in [34], [45], [68], [124], [128], [177]. 

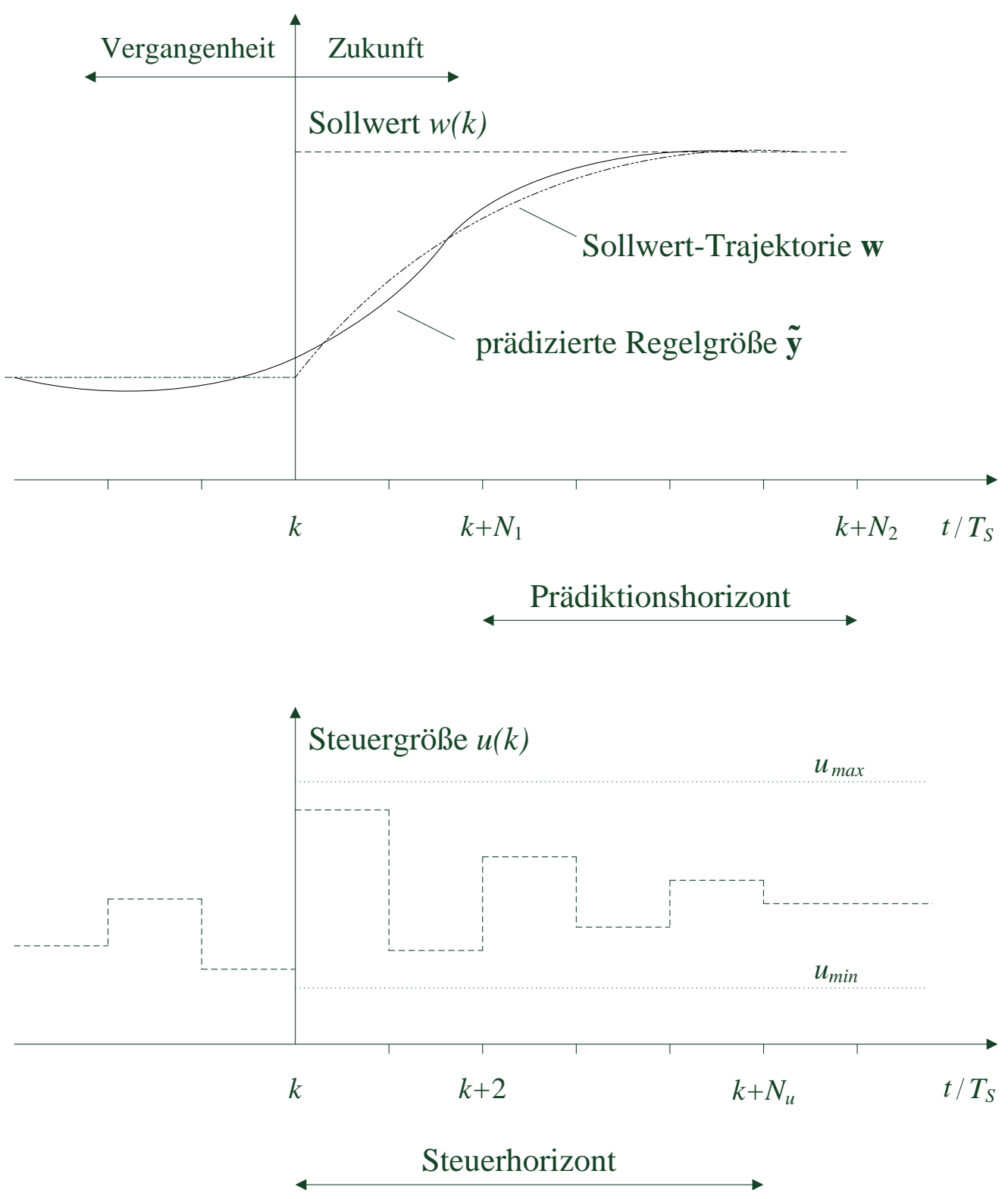

Abbildung 6.2.: Prinzipielle Funktionsweise der prädiktiven Regelung [167].

Der Sollwert $w$ der Regelgröße kann, wie bereits erwähnt, als konstante Größe vorgegeben werden oder er folgt einer bekannten bzw. wie in Abbildung 6.2 gezeigten hinterlegten Sollwerttrajektorie. MPC-Verfahren arbeiten üblicherweise zeitdiskret, die Abtastzeit $T_{S}$ wird dabei als konstant angenommen, $k$ bezeichnet den aktuellen Zeitpunkt. Die prädizierte Regelgröße $\tilde{\mathbf{y}}$ wird vom Prädiktor auf Basis des hinterlegten Prozessmodells, dem Verlauf der Sollwerttrajektorie w und in Abhängigkeit der Stellgrößenänderungen $\Delta u(k)$ bis $\Delta u\left(k+N_{u}\right)$ im Prädiktionshorizont $\left(k+N_{1}\right)$ bis 
$\left(k+N_{2}\right)$ bewertet. $N_{1}$ beschreibt die untere Grenze des Prädiktionshorizonts. Für $N_{1}=0$ beginnt der Prädiktionshorizont mit dem aktuellen Zeitpunkt $k$, er endet nach $\left(k+N_{2}\right)$ Zeitschritten.

$N_{u}$ beschreibt den Steuerhorizont, der auch oft in der Literatur als Stellhorizont bezeichnet wird. Bis zum Zeitpunkt $\left(k+N_{u}\right)$ sind, wie im untereren Bildteil in Abbildung $6.2 \mathrm{zu}$ sehen ist, Stellgrößenänderungen zulässig, danach wird die Stellgröße als konstant angenommen. Dabei gilt $\left(k+N_{u}\right) \leq\left(k+N_{2}\right)$, d.h. der Stellhorizont $N_{u}$ ist immer kleiner oder gleich dem oberen Prädiktionshorizont.

Die bis zum Zeitpunkt $\left(k+N_{u}\right)$ berechneten Stellgrößenänderungen werden allerdings nicht alle auf die Regelstrecke geschaltet. Da das interne Modell der Strecke Ungenauigkeiten aufweist, von außen einwirkende Störungen nicht oder nur unvollständig erfasst werden können, wird, wie bereits in Abbildung 6.1 erläutert, immer nur das erste berechnete Element der Stellgrößenfolge $u(k)$ des Vektors $\mathbf{u}$ auf den Prozess geschaltet. Im nächsten Zyklus wird die Optimierung wiederholt. Durch die erneute Berechnung in jedem Zyklus bewegt sich der Prädiktionszeitraum auf der Zeitachse mit, was in der Literatur auch als gleitender Horizont oder als zurückweichender Horizont (RHC, engl.: Receding Horizon Control) bezeichnet wird.

Bei der Beschreibung des Optimierungskriteriums gibt es verschiedene Möglichkeiten und Ansätze. Gl. (6.1) beschreibt eine quadratische Gütefunktion für den SISOProzess gemäß Abbildung 6.2. In der Gleichung werden die „Kosten“ der Regeldifferenz und des Stelleingriffes bewertet, auf deren Minimierung das prädiktive Regelgesetz basiert. Auch Grenzwerte der Stell- und Regelgröße und andere Nebenbedingungen können als zusätzliche Kriterien eingebracht werden. Dabei ist der Begriff Kosten im Sinne einer betriebswirtschaftlichen Betrachtung zu verstehen. Der Regelungsprozess wird als Optimierungsproblem aufgefasst, das zu lösen ist [167].

$$
\begin{aligned}
J(\Delta \mathbf{u}) & =\sum_{j=N_{1}}^{N_{2}}[w(k+j)-\tilde{y}(k+j)]^{2}+\lambda \sum_{j=0}^{N_{u}} \Delta u^{2}(k+j) \\
& =(\mathbf{w}-\tilde{\mathbf{y}})^{T}(\mathbf{w}-\tilde{\mathbf{y}})+\lambda \Delta \mathbf{u}^{T} \Delta \mathbf{u}
\end{aligned}
$$

mit:

$$
\begin{aligned}
\tilde{\mathbf{y}} & =\left[\tilde{y}\left(k+N_{1} \mid k\right), \tilde{y}\left(k+N_{1}+1 \mid k\right), \ldots, \tilde{y}\left(k+N_{2} \mid k\right)\right]^{T} \\
\mathbf{w} & =\left[w\left(k+N_{1}\right), w\left(k+N_{1}+1\right), \ldots, w\left(k+N_{2}\right)\right]^{T} \\
\Delta \mathbf{u} & =\left[\Delta u(k), \Delta u(k+1), \ldots, \Delta u\left(k+N_{u}\right)\right]^{T}
\end{aligned}
$$


In der Gl. (6.1) beschreibt der Term $[w(k+j)-\tilde{y}(k+j)]$ in diskreter Form die entstehende geschätzte Regeldifferenz auf Basis des zukünftigen Sollwertverlaufes $w(k+j)$ und der prädizierten Regelgröße $\tilde{y}(k+j)$.

Die Bewertung erfolgt im Prädiktionszeitraum $N_{1}$ bis $N_{2}$, d.h. in einem in der Zukunft liegenden Zeitraum. $\Delta u$ beschreibt die Änderung der Stellgröße zwischen zwei Abtastzeitpunkten bis zum Zeitpunkt $N_{u}$. Als zusätzliche Gewichtung kann in der Gl. (6.1) auch die Stellgröße $u$ selbst aufgeführt sein. Laut [110] und [229] führt die Berücksichtigung der Stellgröße allerdings im Allgemeinen zu einer bleibenden Regeldifferenz im stationären Zustand. Der Wert der Stellgrößenänderung $\Delta u$ beeinflusst die stationäre Genauigkeit dagegen nicht. Das Regelungsziel einer Minimierung der Regeldifferenz bei gleichzeitiger Minimierung der Steuergröße stellen in Gl. (6.1) allerdings gegensätzliche Forderungen dar. Entsprechend dem geforderten Regelverhalten können die einzelnen Kostenanteile daher unterschiedlich stark bewertet werden, um die entsprechende Regelziele zu erreichen. In Gl. (6.1) kann die Gewichtung der Stellgrößenänderung mit dem Wert $\lambda$ vorgegeben werden.

Abhängig vom Prozess kann die Forderung nach einem guten Führungsverhalten oder Störverhalten bestehen, es kann aber auch das Ziel sein, Stellenergie einzusparen oder den Stellaufwand zu reduzieren. Die zu optimierende Gütefunktion berücksichtigt alle Regeldifferenzen innerhalb des festgelegten Prädiktionszeitraumes und ermittelt daraus den Vektor $\Delta \mathbf{u}$, der bis zum Zeitschritt $\left(k+N_{u}\right)$ alle Stellgrößenänderungen enthält. Allerdings ist es nicht nötig $\left(k+N_{2}\right)$ Stellgrößenänderungen zu berechnen, da nur die aktuelle Stellgrößenänderung ausgegeben wird. Die Einschränkung der Freiheitsgrade führt zwar zu einer suboptimalen Lösung, der geringe Qualitätsverlust in der Regelgüte kann aber bei einem hinreichend langen Steuerhorizont $\left(N_{u}=\right.$ $3 \ldots 7)$ in praktischen Anwendungen meist in Kauf genommen werden [167].

Das Prinzip der modellprädiktiven Regelverfahren ist sowohl auf lineare als auch auf nichtlineare Prozesse anwendbar. Stellgrößenbeschränkungen, Gln. (6.6), (6.7) und Zustandsbegrenzungen, Gl. (6.8), messbare oder vorhersehbare Störgrößen können im Regelungskonzept berücksichtigt werden.

$$
\begin{aligned}
u_{\min } & \leq u(k) \leq u_{\max } \\
\Delta u_{\min } & \leq \Delta u(k) \leq \Delta u_{\max } \\
\mathbf{x}_{\min } & \leq \mathbf{x}(k) \leq \mathbf{x}_{\max }
\end{aligned}
$$

In der expliziten Berücksichtigung von Begrenzungen bei der Stellsignalgenerierung hat die MPC im Kreis der Regelungsmethoden nahezu ein Alleinstellungsmerkmal 
und erlaubt eine flexible Definition von Regelungszielen [113]. Werden Beschränkungen eingebunden, entsteht ein Optimierungsproblem unter Nebenbedingungen, das nicht mehr analytisch zu lösen ist. Eingangsbeschränkungen begrenzen den Definitionsbereich der Gütefunktion, während Zustandsbegrenzungen den Wertebereich der Prädiktion einschränken [167], [194].

\subsubsection{Dynamic Matrix Control}

Das Dynamic Matrix Control (DMC) Verfahren findet häufig in der verfahrenstechnischen Industrie seine Anwendung. Das Prädiktionsmodell der Ausgangsgröße basiert auf einem zeitdiskreten stabilen Übergangsfunktionsmodell gemäß Gl. (6.9) [167].

$$
\begin{aligned}
\tilde{y}(k+j \mid k) & =\sum_{i=1}^{j} h(i) \Delta u(k+j-i \mid k) \\
& +\sum_{i=j+1}^{m-1} h(i) \Delta u(k+j-i) \\
& +h(m) u(k+j-m) \\
& +d(k+j \mid k) .
\end{aligned}
$$

Der erste Term in Gl. (6.9) beschreibt den Einfluss aktueller und zukünftiger Steuergrößenänderungen, der zweite und dritte Term den Einfluss vergangener Steuergrößenänderungen und die Anfangsbedingung. Der letzte Summand beschreibt die in der Zukunft liegenden Störgrößen $d(k+j \mid k)$. $h(i)$ sind die Gewichte des zeitdiskreten Übergangsfunktionsmodells. In Gl. (6.9) sind die zurückliegenden Steuergrößenänderungen zum Zeitpunkt $k$ bekannt. Die zukünftigen Störsignale werden gemäß Gl. (6.10) prädiziert.

$$
d(k+j \mid k)=y(k)-\sum_{i=1}^{m-1} h(i) \Delta u(k-i)-h(m) u(k+j-m) .
$$

In jeden Abtastschritt wird der aktuelle Fehler für $\epsilon(k)$, Gl. (6.11) zwischen Modellund Prozessgröße, der sich aus den gegebenen Modellungenauigkeiten und Störsignalen ergibt, neu berechnet. 


$$
\epsilon(k)=y(k)-\sum_{i=1}^{m-1} h(i) \Delta u(k-i) .
$$

Für zukünftige Störgrößen wird dabei die Annahme getroffen, dass alle auftretenden Störgrößen gleich der aktuellen Störgröße sind, wie sie sich aus dem Prädiktionsfehler für $\epsilon(k)$ ergeben, d.h. $\epsilon(k+j \mid k)=\epsilon(k)$.

Die prädizierte Ausgangsgröße bzw. der Ausgangsvektor $\tilde{\mathbf{y}}$ ergibt sich für den gesamten Prädiktionshorizont von 1 bis $N_{2}$ und unter Einbeziehen des Steuergrößenvektors $\Delta u$ im Bereich von 1 bis $N_{u}$ in vektorieller Schreibweise gemäß Gl. (6.12).

$$
\tilde{\mathbf{y}}=\mathbf{f}_{D}+\mathbf{H} \Delta \mathbf{u}
$$

Den Namen Dynamic Matrix Control verdankt das Verfahren der dynamischen Matrix H, Gl. (6.13), die den Einfluss der zukünftig zu bestimmenden Steuergrößenänderungen auf die prädizierte Regelgröße $\tilde{\mathbf{y}}$ beschreibt.

$$
\mathbf{H}=\left[\begin{array}{cccc}
h(1) & 0 & \cdots & 0 \\
h(2) & h(1) & \ddots & \vdots \\
\vdots & \vdots & \ddots & 0 \\
h\left(N_{u}\right) & h\left(N_{u}-1\right) & \ddots & h(1) \\
h\left(N_{2}\right) & h\left(N_{2}-1\right) & \cdots & h\left(N_{2}-N_{u}+1\right)
\end{array}\right]
$$

Der freie Anteil $\mathbf{f}_{D}$ in Gl. (6.12) beinhaltet dabei den Einfluss der Prädiktion aus vergangenen Stellgrößenänderungen und dem ermittelten Fehler $\epsilon(k)$, Gl. (6.14). Aufgrund der Beschreibung des Prozesses durch Ein-/Ausgangsmodelle bzw. einzelner Übergangsfunktionen können mit dem DMC-Verfahren nur Regelungen für stabile Prozesse realisiert werden.

$$
\mathbf{f}_{D}=\left[\begin{array}{c}
f(1) \\
\vdots \\
f\left(N_{2}\right)
\end{array}\right] \text { mit: } f(j)=\sum_{i=j+1}^{m-1} h(i) \Delta u(k+j-i)+\epsilon(k)
$$


Die Eigenschaften der Regelung wurden z.B. in [126] untersucht.

\subsubsection{Generalized Predictive Control}

Generalized Predictive Control (GPC) bezeichnet ein prädiktives Regelverfahren auf Basis eines Übertragungsfunktionsmodells mit zusätzlichem Rauschanteil. Der Algorithmus wurde bereits in den 80er-Jahren, erstmals von Clarke [37], [38] für SISOProzesse vorgestellt. Das Regelverfahren nutzt ein CARIMA-Modell (engl.: Controlled Auto-Regressive Integrated Moving-Average), Gl. (6.15), zur Prozessabbildung. A, $B$, und $Z$ stellen Polynome des Verschiebeoperators $z^{-1}$ dar.

$$
\begin{aligned}
y(k) & =\frac{B\left(z^{-1}\right)}{A\left(z^{-1}\right)} u(k)+\frac{Z\left(z^{-1}\right)}{\Delta A\left(z^{-1}\right)} \xi(k) \\
A\left(z^{-1}\right) & =1+a_{1} z^{-1}+\cdots+a_{n a} z^{-n a} \\
B\left(z^{-1}\right) & =b_{0}+b_{1} z^{-1}+\cdots+b_{n b} z^{-n b} \\
Z\left(z^{-1}\right) & =z_{0}+z_{1} z^{-1}+\cdots+z_{n z} z^{-n z} .
\end{aligned}
$$

$\xi$ bezeichnet ein zeitdiskretes weißes Rauschsignal ${ }^{1}$ und $\Delta=1-z^{-1}$ den differenzierenden zeitdiskreten Operator. Das Störgrößenmodell in Gl. (6.15) (zweiter Summand) wird auch als ARIMA-Modell (engl.: Auto-Regressive Integrating MovingAverage) bezeichnet. Durch die spezielle Wahl des Rauschsignalfilters sind alle wichtigen Störfälle wie der autoregressive Prozess (AR) für $z_{0} \xi(k)$, der integrierende Prozess (I) für $1 / \Delta \xi(k)$ und der Prozess mit gleitendem Mittel (MA, engl.: MovingAverage) für $Z\left(z^{-1}\right) \xi(k)$ berücksichtigt.

Das Prozessmodell bzw. das Carima-Modell in Gl. (6.15) kann in die Zustandsraumdarstellung gemäß Gl. (6.16) transformiert werden [167]. Die Systembeschreibung im Zustandsraum kann dabei auch auf Mehrgrößensysteme ausgedehnt werden [167].

$$
\begin{aligned}
\mathbf{x}(k+1) & =\mathbf{A} \mathbf{x}(k)+\mathbf{b} \Delta u(k)+\mathbf{z} \xi(k) . \\
y & =\mathbf{c}^{T} \mathbf{x}(k)+v(k)
\end{aligned}
$$

\footnotetext{
${ }^{1}$ statistisch unabhängiger Zufallsprozess mit Mittelwert Null
} 
Um die Eingangsgröße im Zustandsraum darzustellen, wird $u(k)$ differenziert und ein zusätzlicher Zustand $u(k-1)=u(k)-\Delta u(k)$ eingeführt. Neben der gegebenen Rauschgröße $\xi(k)$ wird ein weiteres Rauschsignal $v(k)$ eingeführt. Es ist gleichfalls als weißes Rauschen moduliert und bildet den Einfluss des Messrauschens auf die Ausgangsgröße $y$ ab. Die prädizierten Ausgangsgrößen für $\tilde{y}(k+j)$ ergeben sich dann gemäß Gl. (6.18). Beide Rauschsignale fließen, bedingt durch ihre Erwartungswerte von Null, in die Prädiktion des Ausgangssignals nicht mit ein. Ist eine Messung des Zustandsvektors $\mathbf{x}(k)$ zu jedem Zeitpunkt $k$ der Prädiktion nicht möglich, ist es notwendig den Zustandsvektor mittels eines Zustandsbeobachters zu bestimmen [110].

$$
\begin{aligned}
\tilde{y}(k+j) & =\mathbf{c}^{T} \mathbf{A}^{j} \mathbf{x}(k)+\sum_{i=1}^{j} \mathbf{c}^{T} \mathbf{A}^{i-1} \mathbf{b} \Delta u(k+j-i) \\
& =\mathbf{F}(j) \mathbf{x}(k)+\sum_{i=1}^{j} \mathbf{H}(i) \Delta u(k+j-i) .
\end{aligned}
$$

Werden die Variablen gemäß der Gln. (6.3), (6.5) zusammengefasst, lässt sich die Vorhersage der Ausgangsgröße $\tilde{\mathbf{y}}$ im Prädiktionshorizont $N_{1}$ bis $N_{2}$ kompakt durch Gl. (6.19) ausdrücken.

$$
\tilde{\mathbf{y}}=\mathbf{F} \mathbf{x}(k)+\mathbf{H} \Delta \mathbf{u} .
$$

Der erste Term in Gl. (6.19) stellt wieder den freien Anteil der Prädiktion dar, der nur vom aktuellen Zustand $x(k)$ abhängt. Der zweite Term beinhaltet den Einfluss des zukünftigen Steuergrößenverlaufs. Die Matrizen $\mathbf{F}$ und $\mathbf{H}$ ergeben sich aus der Modellbeschreibung gemäß Gl. (6.20).

$$
\begin{gathered}
\mathbf{F}=\left[\begin{array}{c}
\mathbf{c}^{T} \mathbf{A}^{N_{1}} \\
\vdots \\
\mathbf{c}^{T} \mathbf{A}^{N_{2}}
\end{array}\right] \quad \mathbf{H}=\left[\begin{array}{ccc}
h\left(N_{1}\right) & \cdots & h\left(N_{1}-N_{u}\right) \\
\vdots & \ddots & \vdots \\
h\left(N_{2}\right) & \cdots & h\left(N_{2}-N_{u}\right)
\end{array}\right] \\
h(i)=\left\{\begin{array}{ll}
\mathbf{c}^{T} \mathbf{A}^{i-1} \mathbf{b} & : i \geq 1 \\
0 & : i<1
\end{array}\right\} .
\end{gathered}
$$


Im GPC-Verfahren wird als Gütefunktional im Ansatz eine quadratische Funktion gemäß Gl. (6.22) gewählt (siehe auch Abschnitt 6.1). $\boldsymbol{\Gamma}$ und $\boldsymbol{\Lambda}$ sind dabei Diagonalmatrizen mit positiven Einträgen. Sie bewerten den Regelfehler und den Einfluss der Stellgrößenänderungen. Die Referenztrajektorie im Prädiktionshorizont wird durch den Vektor w abgebildet.

$$
J(\Delta \mathbf{u})=(\tilde{\mathbf{y}}-\mathbf{w})^{T} \boldsymbol{\Gamma}(\tilde{\mathbf{y}}-\mathbf{w})+\Delta \mathbf{u}^{T} \boldsymbol{\Lambda} \Delta \mathbf{u}
$$

Der optimale Stellgrößenvektor $\Delta \mathbf{u}$ ergibt sich gemäß Gl. (6.23) durch die Minimierung der Kosten mit Hilfe des Least-Squares Verfahrens [167]. Obwohl für den Bereich des Steuerhorizonts mehrere Steuergrößenänderungen berechnet werden, wird wieder nur die aktuelle Steuergrößenänderung $\Delta u(k \mid k)$ für die Regelung ausgegeben, alle anderen Steuergrößen werden verworfen und im nächsten Zyklus bzw. Abtastzeitpunkt wieder neu berechnet.

$$
\Delta \mathbf{u}=\left[\mathbf{H}^{T} \boldsymbol{\Gamma} \mathbf{H}+\boldsymbol{\Lambda}\right]^{-1} \mathbf{H}^{T} \boldsymbol{\Gamma}(\mathbf{w}-\mathbf{F} \mathbf{x}(k)) .
$$

Werden zukünftige Sollwerte in der Berechnung der Steuergröße in Gl. (6.23) berücksichtigt, entspricht das Verfahren einer prädiktiven ausgelegten linearen Zustandsregelung [120].

Sind im Prozessablauf Begrenzungen zu berücksichtigen, muss zur Berechnung von $\Delta \mathbf{u}$ auf das Verfahren der quadratischen Programmierung zurückgegriffen werden [132], [184]. Für den von Clarke vorgestellten GPC-Algorithmus existieren zahlreiche Varianten und Abwandlungen. Einen Überblick liefern [33], [68], [207], [236].

\subsection{Modellbildung der permanentmagneterregten Synchronmaschine}

Für die im Abschnitt 6.3 durchgeführte Simulation für den Vergleich der LMPCRegelung zur PID-Regelung wird die Modelldarstellung der PMSM benötigt. In diesem Abschnitt soll auf die Grundlagen der Modellbildung der PMSM eingegangen werden.

Zur Beschreibung der physikalischen Größen bei Drehfeldmaschinen wird im Allgemeinen, wie bereits in Abschnitt 5.3 erläutert, die Raumzeigerdarstellung verwendet. 
Der Raumzeiger definiert einen Vektor, der sowohl von räumlichen wie auch zeitlichen gerichteten Größen abhängig ist. Eine Einführung zur Raumzeigertheorie für elektrische Maschinen findet sich in [186] und [190].

Wird eine Drehstrommaschine durch ein Maschinenmodell abgebildet, gibt es grundsätzlich zwei Betrachtungsweisen. Die erste Möglichkeit ist, alle Größen bzw. Parameter auf ein feststehendes (statorfestes) $\alpha, \beta$-Koordinatensystem zu beziehen, die andere Möglichkeit ist, alle Größen auf ein sich mitdrehendes $d, q$-Koordinatensystem zu beziehen. Bei der Betrachtung der zweiten Möglichkeit sind alle Gleichungen so zu verstehen, als wenn sich der Betrachter selbst mit dem Rotor bzw. dem $d, q$-System mitbewegt. Je nach Betrachtungsfall müssen Stator- oder Rotorgrößen von einem zum anderen Koordinatensystem umgerechnet bzw. transformiert werden.

\subsubsection{Modell im statorfesten Koordinatensystem}

Ausgehend von Gl. (5.12) kann für die Strangströme der Statorstromraumzeiger, Gl. (6.24), angegeben werden [181]. Gleiches ergibt sich, wenn man die Strangspannungen in einen Spannungsraumzeiger überführt, Gl. (6.25). Der Buchstabe $a$ steht wieder für den komplexen Drehzeiger.

$$
\begin{aligned}
\mathbf{i}(t) & =\frac{2}{3}\left(i_{a}+\mathbf{a} i_{b}+\mathbf{a}^{2} i_{c}\right) . \\
\mathbf{u}(t) & =\frac{2}{3}\left(u_{a}+\mathbf{a} u_{b}+\mathbf{a}^{2} u_{c}\right) .
\end{aligned}
$$

Geht man davon aus, dass die dreiphasige Statorwicklung ideal sinusförmig angeordnet ist, so ergibt sich auch eine sinusförmige verteilte Statorflussverkettung, Gl. (6.26).

$$
\boldsymbol{\psi}(t)=\frac{2}{3}\left(\psi_{a}+\mathbf{a} \psi_{b}+\mathbf{a}^{2} \psi_{c}\right)
$$

Abbildung 6.3 zeigt noch einmal den prinzipiellen Aufbau der zweipoligen PMSM. Die $d$-Richtung des Rotors schließt mit der Statorwicklung a des Drei-Wicklungssystems bzw. mit der $\alpha$-Wicklung des Zwei-Wicklungssystems den Winkel $\vartheta$ ein. In der Raumzeigerschreibweise lässt sich die Statorspannung im statorfesten Koordinatensystem gemäß Gl. (6.27) beschreiben. 


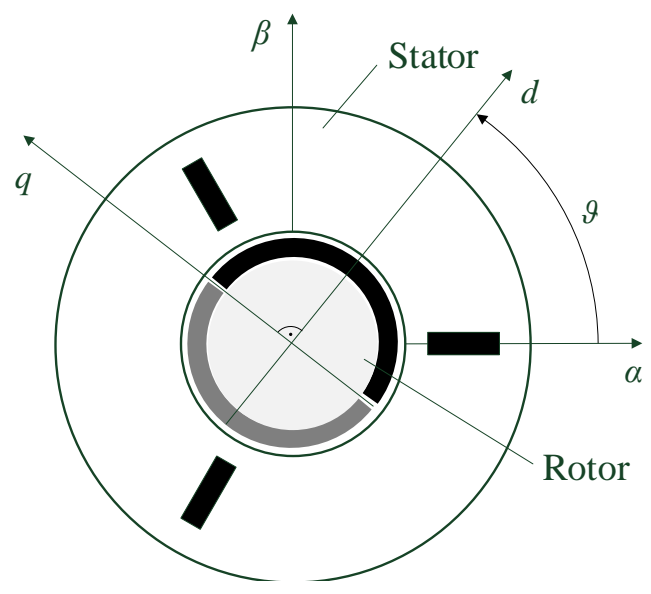

Abbildung 6.3.: Stator- und Rotorkoordinatensystem der PMSM [181].

$$
\mathbf{u}^{s}=R_{s} \mathbf{i}^{s}+\frac{d}{d t} \psi_{s}^{s}
$$

In den folgenden Ausführungen weist ein hochgestellter Index $s$ darauf hin, dass sich die dargestellte Größe auf das statorfeste $\alpha, \beta$-Koordinatensystem bezieht. Ein tiefgestellter Index $s$ bedeutet, dass es sich bei der Größe um eine Statorgröße handelt. Entsprechend bezieht sich ein hochgestellter Index $r$ auf eine Größe im $d, q$ Koordinatensystem - ein tiefgestellter Index $r$ auf eine Rotorgröße.

Der Term $R_{s} \mathbf{i}^{s}$ berücksichtigt den Ohmschen Spannungsabfall an den Statorwicklungswiderständen. Der letzte Term beschreibt die Flussänderung im Stator über der Zeit und somit die entstehende induzierte Spannung. Der Statorfluss $\boldsymbol{\psi}_{s}^{s}$ setzt sich aus zwei Komponenten zusammen, Gl. (6.28).

$$
\boldsymbol{\psi}_{s}^{s}=L_{s} \mathbf{i}^{s}+\boldsymbol{\psi}_{r}^{s}
$$

Zum einen wird ein Fluss im Stator durch den Statorstrom selbst erzeugt $-L_{s}$ steht dabei für die Selbstinduktivität der Statorwicklung - zum anderen liefert $\boldsymbol{\psi}_{r}^{s}$ einen Beitrag des Rotorflusses zur Statorflussverkettung.

Der in der Abbildung 6.3 dargestellte zweipolige Rotor der PMSM besitzt eine magnetische Vorzugsrichtung, die durch die Anordnung der Magnete vorgegeben ist. Eine vom Rotor her die Statorwicklungen durchsetzende Flussverkettung existiert nur in $d$-Richtung. Die magnetische Vorzugsrichtung wird zweckmäßigerweise mit der d-Achse zusammengelegt. Bezogen auf ein mit dem Rotor umlaufendes $d, q$ Koordinatensystem gilt dann Gl. (6.29) [181]. 


$$
\begin{aligned}
& \boldsymbol{\psi}_{r}^{r}=\psi_{r d}+j \psi_{r q} ; \quad \text { da } \psi_{r q}=0: \\
& \boldsymbol{\psi}_{r}^{r}=\psi_{r d}=\psi_{P M} .
\end{aligned}
$$

$\psi_{P M}$ beschreibt den Betrag des magnetischen Flusses bzw. Rotorflusses, der von den Permanentmagneten aufgebracht wird. Um eine Beschreibung der PMSM im statorfesten Koordinatensystem zu erhalten, muss das $d, q$-Koordinatensystem mit umlaufenden Rotorfluss auf das feststehende $\alpha, \beta$-Koordinatensystem umgerechnet werden. Für die Transformationsvorschrift gilt Gl. (6.31) [181].

$$
\begin{aligned}
& \boldsymbol{\psi}_{r}^{s}=\boldsymbol{\psi}_{r}^{r} e^{j \vartheta}=\psi_{P M} e^{j \vartheta} \\
& \boldsymbol{\psi}_{r}^{s}=\psi_{P M} \cos \vartheta+j \psi_{P M} \sin \vartheta=\psi_{P M \alpha}+\psi_{P M \beta} .
\end{aligned}
$$

Die reelle Komponente von $\boldsymbol{\psi}_{r}^{r}$ auf dem sich drehenden $d, q$-Koordinatensystem bildet eine Komponente in $\alpha$ - Richtung $\left(\psi_{P M \alpha}\right)$ und in $\beta$ - Richtung $\left(\psi_{P M \beta}\right)$ im $\alpha, \beta$ Koordinatensystem aus. Aus einer Gleichgröße im $d, q$-Koordinatensystem wird eine Wechselgröße im $\alpha, \beta$-Koordinatensystem. Setzt man Gl. (6.31) in Gl. (6.28) ein und weiterhin Gl. (6.28) in Gl. (6.27) so erhält man Gl. (6.33).

$$
\mathbf{u}^{s}=R_{s} \mathbf{i}^{s}+L_{s} \frac{d}{d t} \mathbf{i}^{s}+j \psi_{P M} e^{j \vartheta} \dot{\vartheta}
$$

Trennt man in Real- und Imaginärteil auf, so ergeben sich die Spannungsgleichungen der PMSM im statorfesten $\alpha, \beta$-Koordinatensystem, Gl. (6.34) und Gl. (6.35).

$$
\begin{aligned}
& u_{\alpha}=\Re\left\{\mathbf{u}^{s}\right\}=R_{s} i_{\alpha}+L_{s} \frac{d}{d t} i_{\alpha}-\psi_{P M} \sin \vartheta \omega . \\
& u_{\beta}=\Im\left\{\mathbf{u}^{s}\right\}=R_{s} i_{\beta}+L_{s} \frac{d}{d t} i_{\beta}+\psi_{P M} \cos \vartheta \omega .
\end{aligned}
$$

In [108] ist ausführlich die Herleitung der Formel für das innere Moment einer allgemeinen zweipoligen Drehfeldmaschine beschrieben. Dabei wird von einem idealen sinusförmigen Verlauf des Strombelags und einem idealen sinusförmigen Verlauf der magnetischen Induktion (Gesamterregung aus Statorerregung und Rotorerregung) entlang des Umfangs der Maschine ausgegangen. Das maximale Drehmoment wird 
erreicht, wenn der Stromzeiger $\mathbf{i}^{s}$ senkrecht auf dem Rotorflusszeiger $\boldsymbol{\psi}_{r}^{s}$ steht, Gl. (6.36).

$$
\begin{aligned}
m_{M} & =\frac{3}{2} p\left(i_{\beta} \psi_{P M \alpha}-i_{\alpha} \psi_{P M \beta}\right) ; \quad \text { mit Gl. (6.32) folgt: } \\
m_{M} & =\frac{3}{2} p \psi_{P M}\left(i_{\beta} \cos \vartheta-i_{\alpha} \sin \vartheta\right) .
\end{aligned}
$$

Der Wert $p$ steht für die Polpaarzahl einer Maschine (hier $p=1$ ). Die Polpaarzahl kann wie eine mechanische Übersetzung aufgefasst werden. Doppelte Polpaarzahl bedeutet doppeltes Moment, aber dann nur halbe Drehzahl an der Motorwelle. Gl. (6.38) beschreibt die Bewegungsgleichung der Maschine unter Berücksichtigung der Gesamtmassenträgheit $J$ [181].

$$
\begin{aligned}
\frac{d}{d t} \omega & =\frac{p}{J}\left(m_{M}-m_{L}\right) \\
\omega_{e l} & =p \omega_{\text {mech }} . \\
\vartheta & =p \vartheta_{\text {mech }} .
\end{aligned}
$$

\section{Signalflussplan der PMSM im statorfesten Koordinatensystem}

Unterzieht man Gl. (6.34) und Gl. (6.35) jeweils der Laplace-Transformation und löst nach $i_{\alpha}$ bzw. $i_{\beta}$ auf, erhält man Gl. (6.41) und Gl. (6.42).

$$
\begin{aligned}
& i_{\alpha}(s)=\left(u_{\alpha}+\psi_{P M} \sin \vartheta \omega\right) \frac{1}{R_{s}+s L_{s}} . \\
& i_{\beta}(s)=\left(u_{\beta}-\psi_{P M} \cos \vartheta \omega\right) \frac{1}{R_{s}+s L_{s}} .
\end{aligned}
$$

Zusammen mit den Gln. (6.37), (6.38), (6.41) und (6.42) lässt sich nun der zeitkontinuierliche Signalflussplan der PMSM, wie in Abbildung 6.4 gezeigt, darstellen (mit $T_{w}=L_{s} / R_{s}$ ). Der Signalflussplan bestätigt anschaulich, wie schon in den Grundgleichungen erkennbar, den nichtlinearen Zusammenhang der Systemgrößen. 


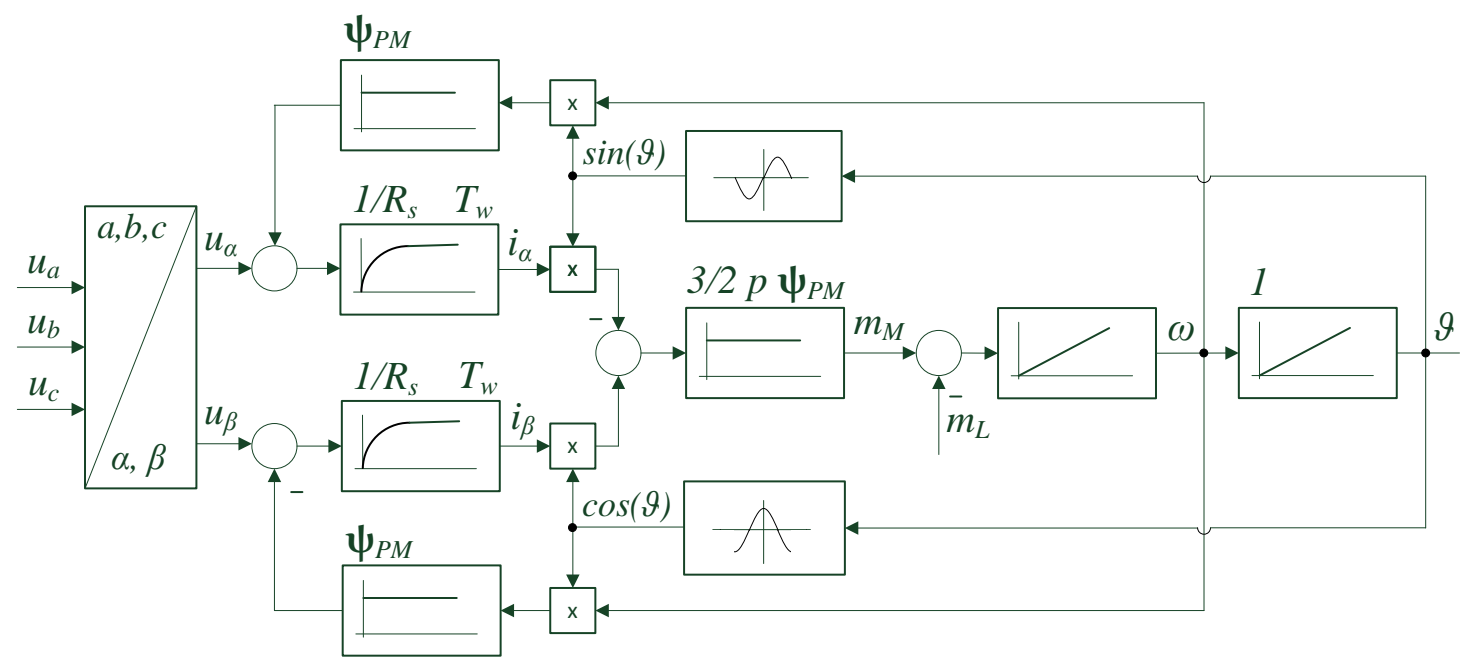

Abbildung 6.4.: Signalflussplan der PMSM im statorfesten Koordinatensystem [190].

\subsubsection{Modell im rotorfesten Koordinatensystem}

Wie in der Abbildung 6.4 zu erkennen ist, ergeben sich im Signalflussplan der PMSM in Statorkoordinaten weniger überschaubare Verhältnisse. Die Größen sind nichtlinear miteinander verknüpft und beinhalten Wechselgrößen. Wird die Beschreibung der PMSM dagegen auf das umlaufende oder rotorfeste Koordinatensystem bezogen, vereinfachen sich die Verhältnisse, da aus den Wechselgrößen im $\alpha, \beta$-Koordinatensystem Gleichgrößen im $d, q$-Koordinatensystem werden. Der Winkel zwischen der $\alpha$-Achse und der $d$-Achse beschreibt dabei die relative Lage der Koordinatensysteme zueinander. Der Winkel wird, wie schon in Abbildung 6.3 gezeigt, mit $\vartheta$ bezeichnet. Ausgangspunkt ist die Spannungsgleichung der Maschine im statorfesten $\alpha, \beta$-Koordinatensystem, Gl. (6.33). Spannung und Strom im Stator werden anhand folgender Koordinatentransformation auf das rotorfeste $d, q$-Koordinatensystem umgerechnet, Gl. (6.43) und Gl. (6.44).

$$
\begin{aligned}
\mathbf{u}^{r} & =\mathbf{u}^{s} e^{-j \vartheta} . \\
\mathbf{i}^{r} & =\mathbf{i}^{s} e^{-j \vartheta} .
\end{aligned}
$$

Für die Umrechnung werden die Werte für Strom und Spannung mit dem komplexen Drehoperator $e^{-j \vartheta}$ multipliziert. Wird weiter der Statorstrom $\mathbf{i}^{s}$ durch den Rotorstrom $\mathbf{i}^{r}$ dargestellt, resultiert daraus Gl. (6.45). 


$$
\mathbf{u}^{r}=R_{s} \mathbf{i}^{r}+L_{s} \frac{d}{d t}\left(\mathbf{i}^{r} e^{j \vartheta}\right) e^{-j \vartheta}+j \omega \psi_{P M}
$$

Werden Raumzeiger, die in einem raumfesten Koordinatensystem beschrieben sind, in ein umlaufendes Koordinatensystem transformiert, macht das prinzipiell keine Schwierigkeiten. Soll die zeitliche Ableitung eines Raumzeigers transformiert werden, bei der die Zeigeramplitude als auch die Zeigerposition im Allgemeinen zeitvariant ist, muss das bei der Differentiation berücksichtigt werden. Dabei ist der komplexe Drehoperator keine elementare Funktion, d.h. bei der Differentiation müssen die Produkt- und Kettenregel angewendet werden. Somit ergibt sich für den mittleren Teil der Gl. (6.45) die Gl. (6.46).

$$
\frac{d}{d t}\left(\mathbf{i}^{r} e^{j \vartheta}\right)=\frac{d}{d t}\left(\mathbf{i}^{r}\right) e^{j \vartheta}+j \omega \mathbf{i}^{r} e^{j \vartheta}
$$

Mit $e^{-j \vartheta} e^{j \vartheta}=1$ folgt die komplexe Spannungsgleichung der Maschine im rotorfesten Koordinatensystem, Gl. (6.47).

$$
\mathbf{u}^{r}=R_{s} \mathbf{i}^{r}+L_{s} \frac{d}{d t} \mathbf{i}^{r}+j \omega L_{s} \mathbf{i}^{r}+j \omega \psi_{P M} .
$$

Trennt man die Gl. (6.47) wieder in Real- und Imaginärteil, erhält man die Spannungsgleichungen (6.48) und (6.49) der Komponenten in $d$ - und q-Richtung.

$$
\begin{aligned}
& u_{d}=R_{s} i_{d}+L_{d} \frac{d}{d t} i_{d}-L_{q} \omega i_{q} . \\
& u_{q}=R_{s} i_{q}+L_{q} \frac{d}{d t} i_{q}+L_{d} \omega i_{d}+\psi_{P M} \omega .
\end{aligned}
$$

Wie in Gl. (6.36) ersichtlich, wird das innere Moment des Motors durch die Komponenten von Fluß und Strom beschrieben. Die Beschreibung lässt sich auch auf das $d, q$-Koordinatensystem übertragen, Gl. (6.50) [157].

$$
m_{M}=\frac{3}{2} p\left[\left(L_{d}-L_{q}\right) i_{d} i_{q}+\psi_{P M} i_{q}\right] .
$$

Für den Fall der PMSM mit oberflächenmontierten Magneten, die hier betrachtet werden soll, vereinfacht sich Gl. (6.50) zu Gl. (6.51). Es gilt $L_{d} \approx L_{q}=L_{s}$. Bei oberflächenmontierten Magneten ist die Asymmetrie des Rotors nur sehr gering (vgl. Abschnitt 6.3.1). 


$$
m_{M}=\frac{3}{2} p \psi_{P M} i_{q}
$$

Für die Bewegungsgleichungen der Maschine gelten natürlich weiterhin die Gln. (6.38), (6.39) und (6.40).

\section{Signalflussplan der PMSM im rotorfesten Koordinatensystem}

Unterzieht man die Gl. (6.48) und Gl. (6.49) jeweils der Laplace-Transformation und löst nach $i_{d}$ bzw. $i_{q}$ auf, folgen die Gl. (6.52) und Gl. (6.53).

$$
\begin{aligned}
& i_{d}(s)=\left(u_{d}+L_{q} \omega i_{q}\right) \frac{1}{R_{s}+s L_{d}} . \\
& i_{q}(s)=\left(u_{q}-L_{d} \omega i_{d}-\psi_{P M} \omega\right) \frac{1}{R_{s}+s L_{q}}
\end{aligned}
$$

Zusammen mit den Gln. (6.38), (6.51), (6.52) und (6.53) lässt sich nun der zeitkontinuierliche Signalflussplan der PMSM für ein rotorfestes Koordinatensystem darstellen. Mit den Zeitkonstanten $T_{d}=L_{d} / R_{s}$ und $T_{q}=L_{d} / R_{s}$ folgt Abbildung 6.5.

Im Signalflussplan treten, wie schon in den Gleichungen ersichtlich, keine Wechselgrößen mehr auf. Die Vereinfachung wurde erkauft durch die Verkopplung der $i_{d}$ und $i_{q}$ Stromkomponenten.

\subsection{Bewertung der modellprädiktiven Regelung}

Viele Regelungen im Bereich der industriellen Anwendungen für elektrische Antriebe beruhen immer noch auf konventionellen PID-Regelalgorithmen. Für die Berechnung der Stellgröße beim PID-Regler werden aktuelle Messwerte herangezogen, ein Prozessmodell wird nicht berücksichtigt. Das Einhalten von Beschränkungen kann im Regelverfahren schwer oder gar nicht garantiert werden.

Modellprädiktive Regelverfahren nutzen ein Prozessmodell zur Berechnung der optimalen Steuergrößen anhand bisheriger Mess- und Zustandswerte und zukünftiger prädiktiver Werte der Regelgrößen. Das Prozessmodell wird im Reglerentwurf berücksichtigt bzw. bildet die Basis der Regelung. Prozessbeschränkungen, Stellgrößenund Zustandsbeschränkungen können vorgegeben werden und können in das Ziel der Regelung, z.B. mit welcher Dynamik das Regelziel erreicht werden soll, eingebunden 


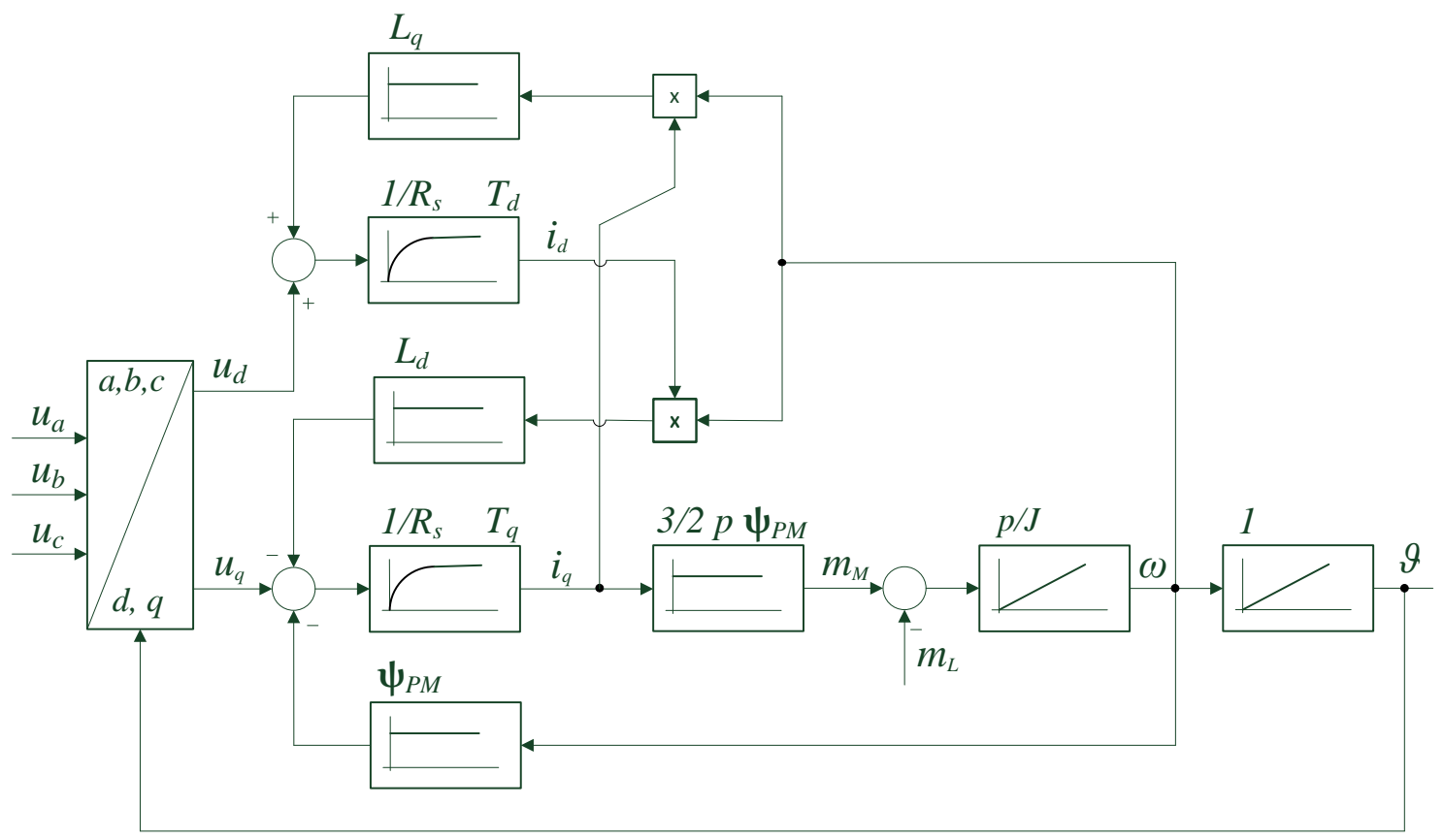

Abbildung 6.5.: Signalflussplan der PMSM im rotorfesten Koordinatensystem [190].

werden. Zusätzlich kann bei Mehrgrößensystemen eine Kopplung zwischen den unterschiedlichen Regelgrößen berücksichtigt werden, was bei einer PID-Regelung nicht möglich ist.

Allerdings benötigen MPC-Regler gegenüber PID-Regelungen und anderen verwandten Regelverfahren, bedingt durch den Prädiktions- und Optimierungsaufwand, eine erhebliche Rechenleistung, was die praktische Umsetzung auf Standardrechnersysteme erschwert. Die Implementierung in ein Echtzeitsystem, besonders bei Regelungssystemen mit kleinen Zeitkonstanten, erhöht die Anforderungen zusätzlich.

In den folgenden Abschnitten wird simulativ untersucht, welche Vorteile eine modellprädiktive Regelung gegenüber einem klassischen PID-Regelverfahren aufweist. Die Simulation für beide Regelverfahren wurde in MATLAB mit der Version R2017a realisiert. Neben Simulink werden speziell zur Simulation der modellbasierten Regelung die Control System Toolbox und die MPC-Toolbox (engl.: Model Predictive Control) eingesetzt. 


\subsubsection{Modellbildung im Zustandsraum}

Der Ausgangspunkt für die Simulationsdurchführung in der vorliegenden Arbeit bildet das nichtlineare zeitkontinuierliche Modell der PMSM entsprechend Abschnitt 6.2.2 im rotorfesten Koordinatensystem. Der elektrische Teil der PMSM lässt sich durch die Gln. (6.48) und (6.49) beschreiben. Im weiteren Verlauf soll die PMSM als Vollpolsynchronmaschine betrachtet werden, deren Rotor durch oberflächenmontierte Magneten aufgebaut ist. Hier liegt die relative Permeabilität der eingesetzten Magnetwerkstoffe im Bereich von Luft $\left(\mu_{r}=\mu_{M} / \mu_{0} \approx 1\right)$. Die Anordnung der Magnete wirkt dabei wie ein vergrößerter und gleichmäßiger Luftspalt. Entlang des Luftspaltes ist keine oder nur eine sehr geringe Induktivitätsänderung vorhanden [157].

Für das Grundwellenmodell der Maschine und für die spätere weitere Betrachtung werden für die Längs- und Querachse deshalb hier keine unterschiedlichen Induktivitäten betrachtet, d.h. $L_{d} \approx L_{q}=L_{s}$ [157]. Ferner werden die Statorinduktivitäten $L_{d}$ und $L_{q}$ bzw. $L_{s}$ sowie der Statorwiderstand $R_{s}$ und der Erregerfluss $\psi_{P M}$ als konstant angenommen. Für die elektrische Winkelgeschwindigkeit gilt: $\omega_{e l}=p \omega_{\text {mech }}$, mit $p=5$.

Ein weiteres elektrisches Teilsystem bildet der Wechselrichter. Unter Berücksichtigung der Aussteuergrenzen lässt sich der Wechselrichter als Totzeitglied mit Totzeit $T_{t}$ und einer Verstärkung von $V=1$ beschreiben [190]. Die Größen $u_{d}$ und $u_{q}$ werden daher in Gln. (6.48) und (6.49) durch die Größen $u_{d, w}$ und $u_{q, w}$ durch die Gln. (6.54) und (6.55) dargestellt.

$$
\begin{aligned}
& u_{d, w}(t)=u_{d}\left(t-T_{t}\right) . \\
& u_{q, w}(t)=u_{q}\left(t-T_{t}\right) .
\end{aligned}
$$

Zuletzt beschreiben die Gln. (6.56) bis (6.58) den mechanischen Teil der PMSM mit dem Einfluss des Lastmomentes.

$$
\begin{aligned}
m_{M} & =\frac{3}{2} p\left[\left(L_{d}-L_{q}\right) i_{d} i_{q}+\psi_{P M} i_{q}\right] . \\
\dot{\omega}_{m e c h} & =\frac{1}{J_{M}+J_{L}}\left(m_{M}-m_{L}\right)=\frac{1}{J_{g e s}}\left(m_{e l}-m_{L}\right) . \\
\dot{\vartheta}_{\text {mech }} & =\omega_{\text {mech }} .
\end{aligned}
$$


Die Gln. (6.54) bis (6.58) lassen sich übersichtlich in einem Zustandsraummodell darstellen bzw. zusammenfassen. Gl. (6.59) beschreibt das nichtlineare zeitkontinuierliche Modell der PMSM mit allen Systemgrößen. Der Vektor $\mathbf{x}_{n l}=\left[i_{d}, i_{q}, \omega_{\text {mech }}, \vartheta_{\text {mech }}\right]^{T}$ beschreibt dabei die Zustände des Systems, der rechte Spaltenvektor den Einfluss des nicht messbaren Lastmoments $m_{L}$ als Störgröße. Die Eingangsgrößen werden durch den Spannungsvektor $\mathbf{u}=\left[u_{d}, u_{q}\right]^{T}$ abgebildet.

Zur Simulation der Regelungseigenschaften der Strecke mit der MPC-Toolbox ist es notwendig, das nichtlineare zeitkontinuierliche Zustandsraummodell zu linearisieren. Die Nichtlinearitäten im Streckenmodell sind dabei im elektrischen Teil (für $L_{d}=L_{q}$ ) gegeben.

$$
\left[\begin{array}{c}
i_{d} \\
i_{q} \\
\omega_{\text {mech }} \\
\vartheta_{\text {mech }}
\end{array}\right]=\left[\begin{array}{c}
-\frac{R_{s}}{L_{d}} i_{d}+\frac{L_{q}}{L_{d}} p i_{q} \omega_{m e c h} \\
-\frac{R_{s}}{L_{q}} i_{q}-\frac{L_{d}}{L_{q}} p i_{d} \omega_{m e c h}-\frac{\psi_{P M}}{L_{q}} p \omega_{m e c h} \\
\frac{3 p}{2 J_{g e s}}\left[\left(L_{d}-L_{q}\right) i_{d} i_{q}+\psi_{P M} i_{q}\right] \\
\omega_{\text {mech }}
\end{array}\right]+\left[\begin{array}{c}
\frac{1}{L_{d}} u_{d, w} \\
\frac{1}{L_{q}} u_{q, w} \\
0 \\
0
\end{array}\right]+\left[\begin{array}{c}
0 \\
0 \\
-\frac{1}{J_{g e s}} m_{L} \\
0
\end{array}\right]
$$

Durch den prädiktiven Charakter des MPC-Reglers ist es möglich, Stellgrößenbeschränkungen, messbare oder modellierbare Störungen sowie Totzeiten im System zu erkennen und zu berücksichtigen. Durch eine Störgrößenaufschaltung wird der Einfluss der Nichtlinearitäten dabei reduziert.

$$
\dot{\mathbf{x}}_{\text {lin }}=\left[\begin{array}{c}
i_{d} \\
i_{q} \\
\omega_{\text {mech }} \\
\vartheta_{\text {mech }}
\end{array}\right]=\left[\begin{array}{cccc}
-\frac{R_{s}}{L_{d}} & 0 & 0 & 0 \\
0 & -\frac{R_{s}}{L_{q}} & -\frac{\psi_{P M}}{L_{q}} p & 0 \\
0 & \frac{3 p}{2 J_{g e s}} \psi_{P M} & 0 & 0 \\
0 & 0 & 1 & 0
\end{array}\right] \mathbf{x}_{l i n}+\left[\begin{array}{c}
\frac{1}{L_{d}} u_{d, w} \\
\frac{1}{L_{q}} u_{q, w} \\
0 \\
0
\end{array}\right]+\left[\begin{array}{c}
0 \\
0 \\
-\frac{1}{J_{g e s}} m_{L} \\
0
\end{array}\right] .
$$

Für das linearisierte Modell stellt die Toolbox eine ausführliche Bibliothek und Dokumentation zur Verfügung [22]. MPC-Algorithmen, welche lineare Modelle betrachten, werden als Linear Model Predictive Control (LMPC) bezeichnet. Für $L_{d}=L_{q}$ erfolgt die Linearisierung um den Arbeitspunkt $\mathbf{x}_{0}=0$. Das linearisierte Zustandsraummodell der Regelstrecke gemäß Gl. (6.60) folgt nach Durchführung einer Taylorreihenentwicklung um $\mathbf{x}_{0}$ mit Abbruch nach dem linearen Glied. Ergänzende Informationen 
zu MPC-Verfahren und die Auswahl geeigneter applikationsabhängiger Modelle findet sich in [34] und [45].

\subsubsection{Linear Model Predictive Control Algorithmus}

Die MPC-Toolbox von MATLAB bietet die Möglichkeit, Linear Model Predictive Control (LMPC) Algorithmen für modellprädiktive Regler zu entwerfen, zu analysieren und zu simulieren. Neben der MPC-Toolbox ist die Control System Toolbox ein wichtiger und notwendiger Bestandteil für die Modellbildung und den Reglerentwurf. Innerhalb der Simulink-Umgebung ist die Modellierung von allgemeinen linearen und nichtlinearen Modellen mit Hilfe graphischer Blöcke möglich. Grundsätzlich können mit der MPC-Toolbox selbst nur lineare, zeitinvariante Systeme (LTI-Systeme) behandelt werden. MIMO-Systeme (engl.: Multi-Input Multi-Output) werden ebenfalls unterstützt.

Die in der MPC-Toolbox entwickelten modellprädiktiven Regler für lineare Systeme lassen sich in die Simulink-Umgebung exportieren. Nach der Implementierung können dadurch zugehörige nichtlineare Systeme getestet werden. Prädiktions- und Steuerhorizont sowie Gewichtsfaktoren hinsichtlich der Regelziele können als Regelparameter vorgeben werden. Neben der Vorgabe von Konstant-Sollwerten ist es auch möglich, eine Referenztrajektorie zu hinterlegen. Die Systeme bzw. Modelle müssen in diskretisierter Form vorliegen. Die Gl. (6.61) beschreibt das Zustandsraummodell für ein lineares zeitdiskretes System [22].

$$
\begin{aligned}
\mathbf{x}_{k+1} & =\mathbf{A} \mathbf{x}_{k}+\mathbf{B}_{u} \mathbf{u}_{k}+\mathbf{B}_{v} \mathbf{v}_{k}+\mathbf{B}_{d} \mathbf{d}_{k} \\
\mathbf{y}_{k} & =\mathbf{C} \mathbf{x}_{k}+\mathbf{D}_{v} \mathbf{v}_{k}+\mathbf{D}_{d} \mathbf{d}_{k} .
\end{aligned}
$$

Der Vektor $\mathbf{v}_{k}$ in der Gl. (6.61) beschreibt die messbaren Störungen, der Vektor $\mathbf{d}_{k}$ die nicht messbaren Störungen. Die Strecke ist um ein Störmodell erweitert. Die Zustandsschätzung bzw. die Korrektur erfolgt mit einem linearen Kalman-Filter [131].

Für Modelle ohne Beschränkungen stellt die MPC-Toolbox den GPC-Algorithmus zur Verfügung (siehe Abschnitt 6.1.2). Treten im Modell Beschränkungen auf, wird das Kostenfunktional, Gl. (6.62), in jedem Schritt online unter Beachtung der Beschränkungen gelöst [131], [151]. $u(k+m-l)$ bezeichnet die $k+m-l$-te Steueranweisung, $y(k+l \mid k)$ die $l$-te prädizierte Regelgröße zum $k$-ten Abtastschritt, $\mathbf{Q}$ und $\mathbf{R}$ sind Gewichtsmatrizen (siehe auch Abschnitt 6.1). 


$$
\begin{aligned}
J(\Delta \mathbf{u}) & =\underset{\Delta u(k) \ldots \Delta u(k+m-l)}{\min _{l=1}}\left(\sum_{l=1}^{p}|\mathbf{Q}(y(k+l \mid k)-w(k+l))|^{2}\right. \\
& \left.+\sum_{l=1}^{m}|\mathbf{R} \Delta u(k+l-1)|^{2}\right) .
\end{aligned}
$$

Zur Lösung des Problems verwendet die MPC-Toolbox die Dantzig ${ }^{2}$-Wolfe ${ }^{3}$-Methode der aktiven Mengen [61]. Dabei wird das Optimierungsproblem auf ein quadratisches Problem überführt, Gl. (6.63), das bezüglich $x$ minimiert wird. Die Berechnung der Lösung erfolgt iterativ.

$$
J(\mathbf{x})=\min _{x}\left(\frac{1}{2} \mathbf{x}^{T} \mathbf{H}_{f} \mathbf{x}+\mathbf{f}^{T} \mathbf{x}\right)
$$

\subsubsection{Parameter des modellprädiktiven Regelverfahrens}

Für den LMPC-Algorithmus lassen sich in der MPC-Toolbox, wie bereits beschrieben, verschiedene Parameter mit Randbedingungen und Beschränkungen angeben. Der Prädiktionshorizont definiert das Zeitintervall, mit dem das zukünftige Verhalten der Regelstrecke, basierend auf dem mathematischen Modell der Strecke und dem aktuellen Zustand, prädiziert wird. Der Wert bzw. die Länge des Prädiktionshorizonts ist so zu wählen, dass Einschwingvorgänge der Regelstrecke in die Vorhersage eingebunden werden und Verletzungen der Randbedingungen und Beschränkungen rechtzeitig erkannt werden.

Für die Abtastzeit des MPC-Reglers wurde in der durchgeführten Simulation ein Wert von $T_{S}=1 \mathrm{~ms}$ gewählt. Die Abtastzeit richtet sich in erster Linie nach der Streckenantwortzeit bzw. nach der größten Zeitkonstante des Stromregelkreises. Der Prädiktionshorizont ist in der Simulation mit $H_{P}=100$ vorgegeben. Für die gegebene Abtastzeit ist so das Verhalten der Regelstrecke für einen Zeitraum von $T_{P}=0.1 s$ prädizierbar. Für die Wahl des Steuerhorizonts $H_{C}$ hat sich für die vorliegende Regelstrecke ein Wert für $H_{C}=2$ als ausreichend gezeigt. Die Werte wurden dabei empirisch ermittelt. Die ermittelten Parameter stellen einen Kompromiss aus Abtastzeit, Prädiktionshorizont und der daraus resultierenden Rechenleistung für das Optimierungsproblem dar. Weitere Reglerparameter bzw. Gewichtsfaktoren der LPMC-Regelung sind im Abschnitt C.1 aufgeführt.

\footnotetext{
${ }^{2}$ George Bernard Dantzig, US-amerikanischer Mathematiker

${ }^{3}$ Philip Wolfe, US-amerikanischer Mathematiker
} 
Mit der MPC-Toolbox ist es möglich, Beschränkungen von Stellgrößen, Stellgrößenänderungen und Ausgangsgrößen als Teil der Optimierung zu berücksichtigen. Die Randbedingungen können als Soft-Constraints oder auch als exakt zu erfüllende Hard-Constraints eingebunden werden. In der durchgeführten Simulation sind aktuell keine Beschränkungen der Stellgrößen vorgegeben. Allerdings wird der Motor auch nicht an der Drehmomentgrenze betrieben.

Alle Eingangs- und Ausgangsgrößen sind im MPC-Tool zur besseren Übersicht und Parametrierbarkeit im Intervall [-1, 1] skaliert. Neben den bekannten messbaren Störungen kann man in der MPC-Toolbox auch unbekannte Störungen auf das System einwirken lassen. Die Störungen können konstant, als Sprung, gepulst, linear, sinusförmig oder auch gaußverteilt vorgegeben werden. Im Fall des nicht messbaren Lastmomentes wird eine sprungförmige Störgröße aufgeschaltet.

Um den Drehzahlfehler zu minimieren, wird für die Wahl der Gewichtungsfaktoren im Gütemaß die Abweichung der Referenzdrehzahl um den Faktor 10 stärker gewichtet als die Änderung der Stellgröße. Der drehmomentbildende q-Strom wird durch die Vorgabe der Referenzdrehzahl dabei indirekt berücksichtigt.

\subsubsection{Darstellung der Ergebnisse der modellprädiktiven Regelung}

Für die Bewertung der Regelungseigenschaften des MPC-Algorithmus im Vergleich zu einer klassischen PID-Kaskadenregelung wurde ein simulativer Vergleich zwischen beiden Regelungsverfahren durchgeführt. In Abbildung 6.6 ist das Blockschaltbild der Kaskadenregelung aus Strom- und Drehzahlregelung schematisch dargestellt, wie es für die Simulation verwendet wird (vgl. auch Abschnitt 5.3.5).

Das Blockschaltbild entspricht dem klassischen Ansatz für viele Anwendungsfälle in der Industrie im Bereich der Antriebstechnik. Wie bereits in Abschnitt 5.3.3 beschrieben, besteht die Stromregelung aus zwei PI-Reglern $G_{R i, d}(s)$ und $G_{R i, q}(s)$, die den Strom im $d$ - und $q$-Zweig separat regeln. Die Einstellung der Stromregler erfolgt nach dem Kriterium des Betragsoptimums. Die Messwertfilter für die Strommessung $G_{F i}(s)$ und die Drehzahlmessung $G_{F \omega}(s)$ sind als $\mathrm{PT}_{1}$-Glieder berücksichtigt.

Wie in Abschnitt 5.3.3 gezeigt, ist bei der FOC das zur Verfügung gestellte Drehmoment des Motors nur vom q-Strom abhängig. Der d-Strom wird für die im Modell verwendete PMSM auf $i_{d}=0$ geregelt. Der Drehzahlregler $G_{R \omega}(s)$ ist gleichfalls als PI-Regler ausgelegt und ist nach dem Kriterium des symmetrischen Optimum ausgelegt. Um ein zu starkes Überschwingen bei einer Führungsgrößenänderung zu vermeiden, wird zusätzlich ein Führungsgrößenfilter $G_{\omega}(s)$ integriert. Die Einstellwerte der Regler und Messwertfilter sind in Abschnitt C.2 beschrieben. 


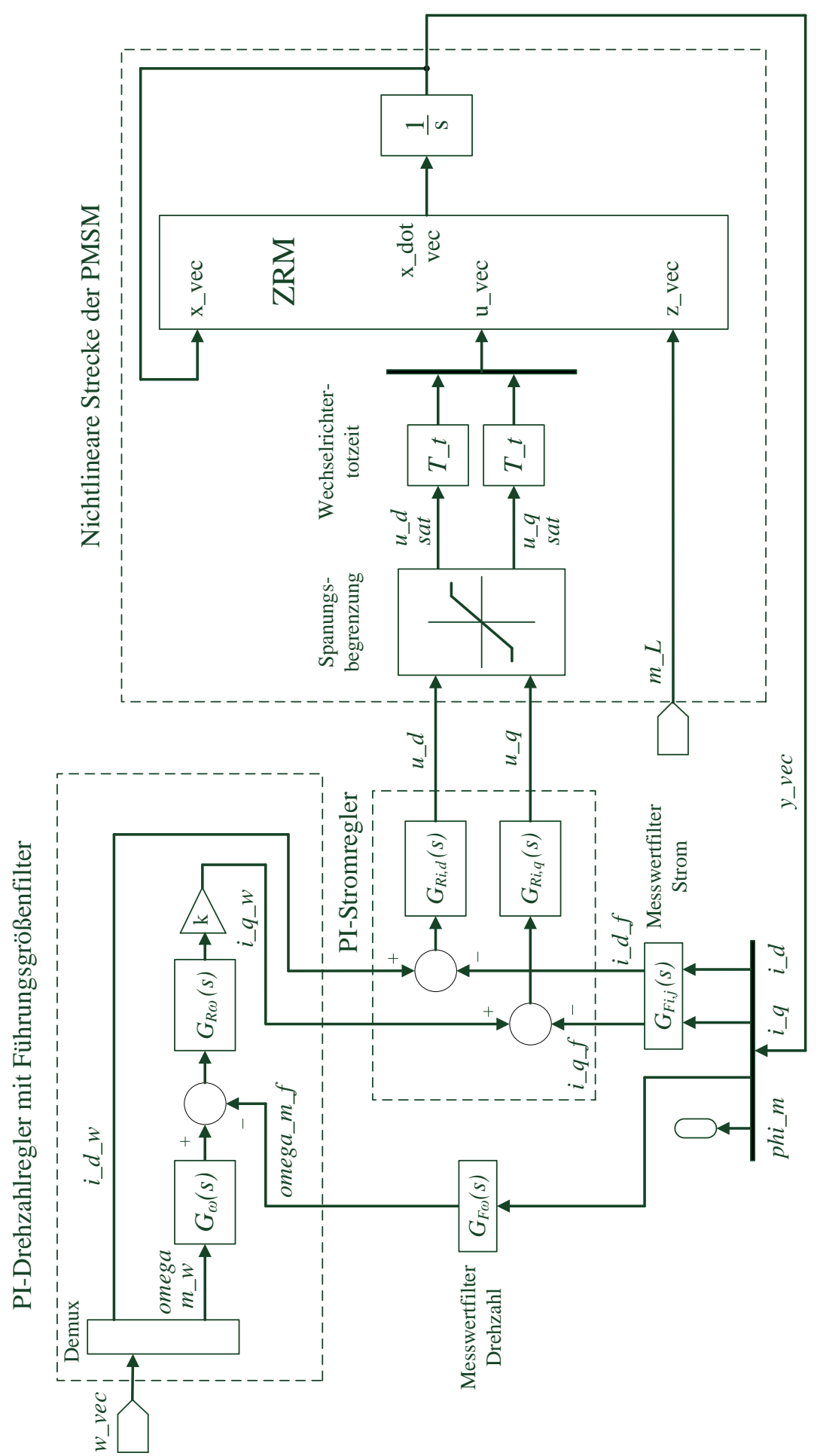

Abbildung 6.6.: Blockschaltbild für die Simulation der Kaskadenregelung. 
Dem Wechselrichter ist es nicht möglich, beliebig große Stellspannungen zu erzeugen. In Abbildung 6.6 sind die Spannungen für $u_{d}$ und $u_{q}$ daher begrenzt. Da $u_{d}$ und $u_{q}$ im rotorfesten Koordinatensystem immer senkrecht zueinander stehen, gelten für die Begrenzungen die Gln. (6.64) und (6.65) [190].

$$
\begin{aligned}
& \left|u_{d}\right| \leq \sqrt{\left|u_{\max }^{2}-u_{q}^{2}\right|} \\
& \left|u_{q}\right| \leq \sqrt{\left|u_{\max }^{2}-u_{d}^{2}\right|} .
\end{aligned}
$$

$u_{\max }$ definiert in den Gln. (6.64) und (6.65) die maximale Amplitude des Spannungsraumzeigers (vgl. Abbildung C.2). Die Wechselrichtertotzeiten im $d$ - und $q$-Zweig sind durch $T_{t}$ berücksichtigt. Das nichtlineare Zustandsraummodell (ZRM) entspricht Gl. (6.59) für $L_{d}=L_{q}$ (siehe auch Abbildung C.1).

Abbildung 6.7 zeigt das Blockschaltbild mit LMPC-Ansatz. Der modellbasierte MPCAlgorithmus übernimmt hier in geschlossener Form die Drehzahl- und Stromregelung. Das nichtlineare Streckenmodell der PMSM mit Spannungsbegrenzung, Wechselrichtertotzeit und Zustandsraummodell entspricht der Abbildung 6.6 bzw. der PIDRegelung.

Die Grundlage für den Reglerentwurf bildete das linearisierte Modell entsprechend Gl. (6.60). Durch die Linearisierung um den Arbeitspunkt $\mathbf{x}_{0}=0$ sind die Terme $\left(L_{q} / L_{d}\right) p i_{q} \omega_{\text {mech }}$ und $\left(L_{d} / L_{q}\right) p i_{d} \omega_{\text {mech }}$ entfallen, was die Modellgüte verschlechtert. Da $i_{d}, i_{q}$ und $\omega_{\text {mech }}$ messbare Größen sind, können sie jedoch in Form einer Störgrößenaufschaltung für die Optimierung berücksichtigt werden. Im Simulationsmodell (Abbildung 6.7) ist der additiven Störterm $\mathbf{z}_{\text {Stoer }}$ gemäß Gl. (6.66) auf den Eingang des MPC-Reglers als messbare Störgröße aufgeschaltet.

$$
\mathbf{z}_{\text {Stoer }}=\left[\frac{L_{q}}{L_{d}} p i_{q} \omega_{m e c h}, \frac{L_{d}}{L_{q}} p i_{d} \omega_{m e c h}, 0,0\right]^{T}
$$

Die Abbildungen 6.8 und 6.9 zeigen das simulierte Verhalten des LMPC-Algorithmus im Vergleich zur PID-Regelung für unterschiedliche Führungsgrößen und Führungsgrößensprünge.

Die beiden obersten Bilder in den Abbildungen zeigen je den Verlauf der Drehzahl $n_{P I D}$ der PID-Regelung und den Verlauf der Drehzahl $n_{M P C}$ der LMPC-Regelung. Der Verlauf der Drehzahl der LMPC-Regelung $n_{M P C}$ in beiden Abbildungen (Kennzeichnung in blauer Farbe) ist in etwa deckungsgleich mit dem Verlauf der Drehzahl 


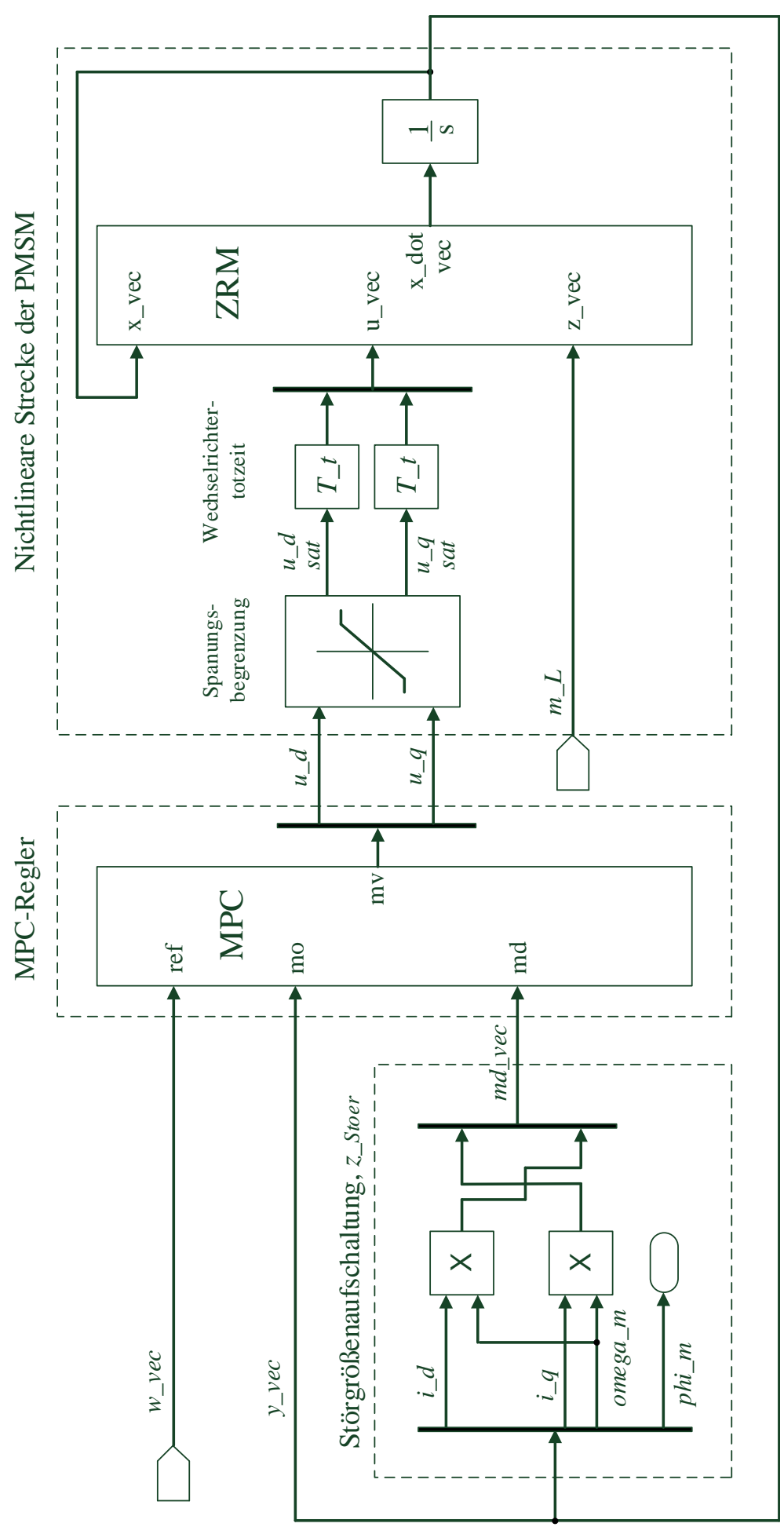

Abbildung 6.7.: Blockschaltbild Simulation MPC-Regelung. 

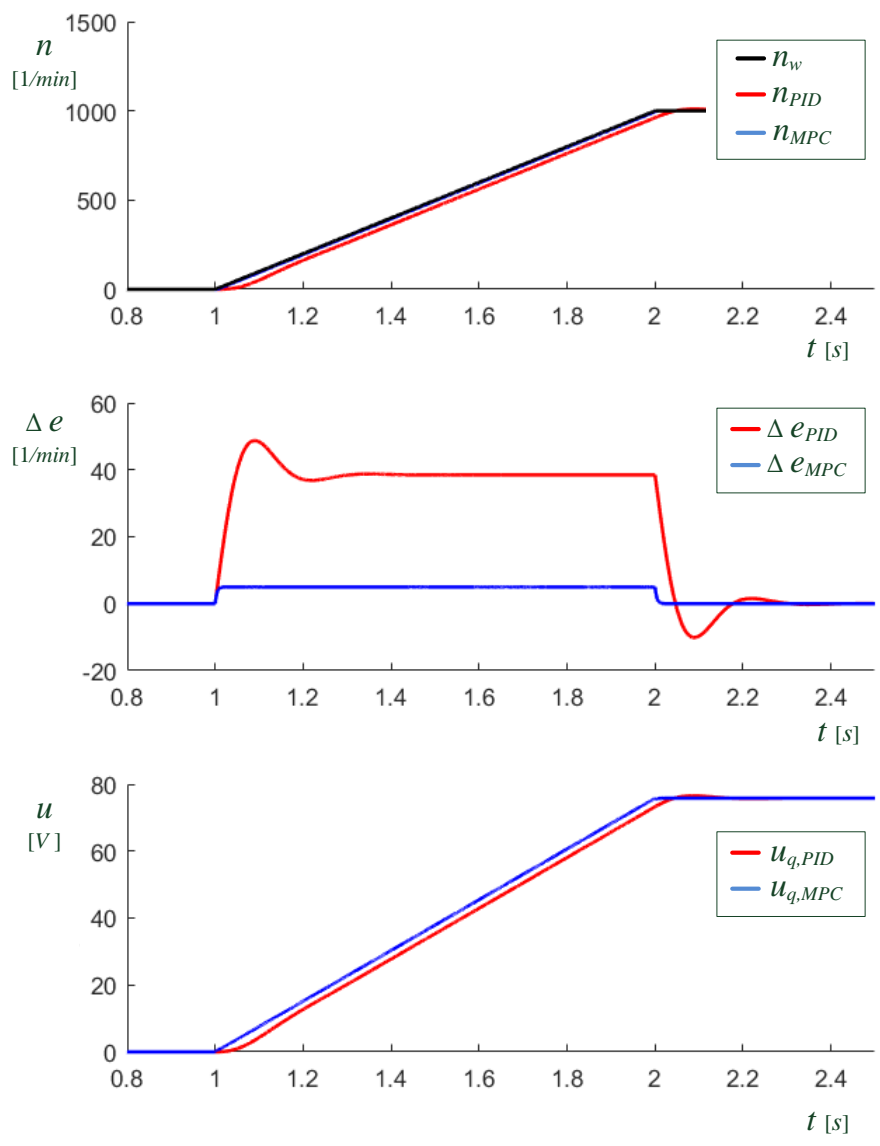

Abbildung 6.8.: Vergleich Führungsverhalten LMPC/PID für kleine Drehzahlen.

des PID-Reglers und deshalb auf dem Bild nicht eindeutig zu erkennen. Der vorgegebene Referenzdrehzahlsollwert ist im Bild mit $n_{w}$ bezeichnet.

In Abbildung 6.8 ist für beide Reglereingänge zeitgleich eine Drehzahlrampe mit der Steigung von $\Delta n=1000(1 / \mathrm{min}) / s$ vorgegeben. In Abbildung 6.9 ist die Anstiegsgeschwindigkeit der Drehzahlrampe größer und beträgt $\Delta n=3500(1 / \mathrm{min}) / \mathrm{s}$. In den beiden mittleren Bildern ist je der ermittelte Drehzahlfehler bzw. Schleppfehler der PID-Regelung: $\Delta e_{P I D}=\left(n_{w}-n_{P I D}\right)$ und der LMPC-Regelung: $\Delta e_{M P C}=$ $\left(n_{w}-n_{M P C}\right)$ dargestellt. Die beiden unteren Bilder in den Abbildungen 6.8 und 6.9 zeigen die unterschiedlichen Stellgrößenänderungen $u_{q, P I D}$ und $u_{q, M P C}$ für die verschiedenen Arbeitspunkte.

Wie im mittleren Bild in Abbildung 6.8 gut zu erkennen ist, ist der Drehzahlfehler beim LMPC-Regler während des Hochlaufens des Motors wesentlich geringer als beim PID-Regler. Auch zeigt der PID-Regler beim Start und beim Übergang in den stationären Zustand ein deutlich stärkeres Einschwingverhalten. Das Verhalten verstärkt sich bei größeren Drehzahlsprüngen, was in Abbildung 6.9 deutlich an der 

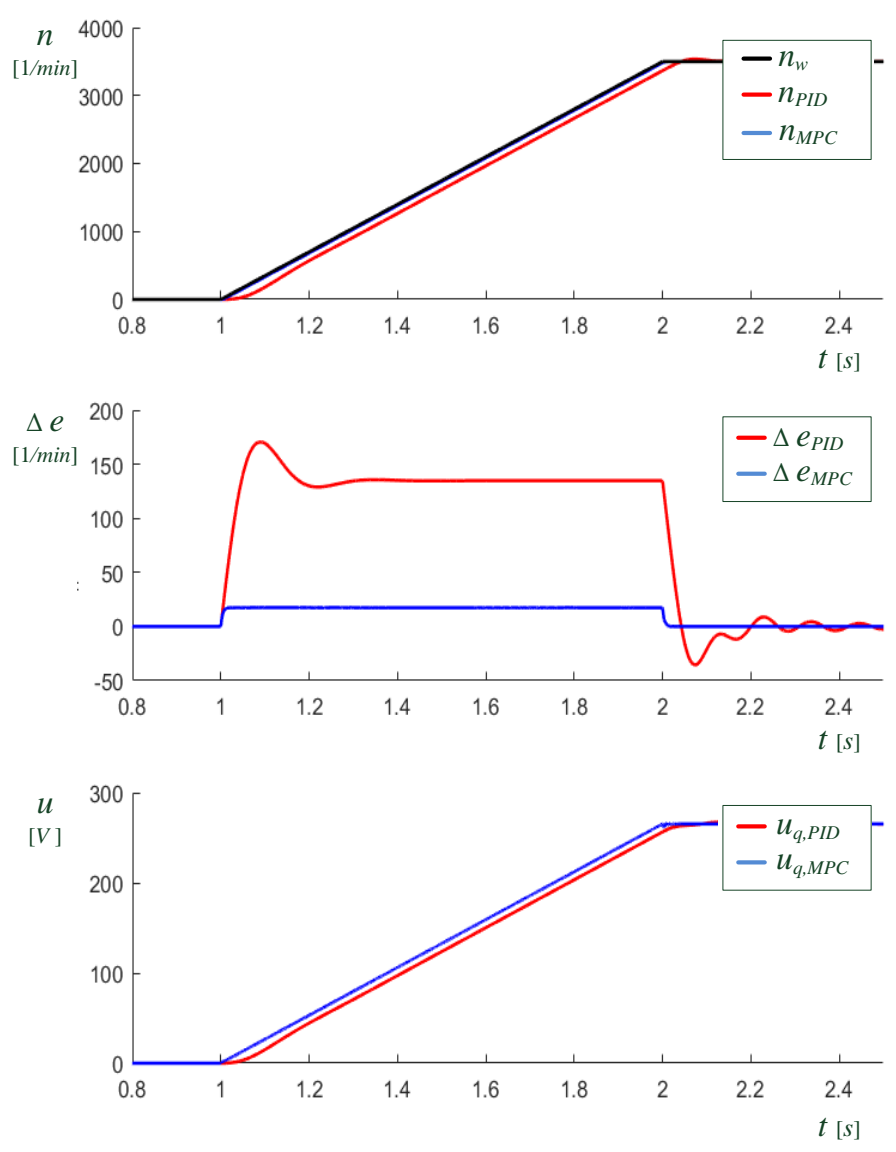

Abbildung 6.9.: Vergleich Führungsverhalten LMPC/PID für große Drehzahlen.

zunehmenden Drehzahlwelligkeit zu erkennen ist. Der Vorteil der prädiktiven Regelung ist hier die Kenntnis und das Einbeziehen des Streckenverhaltens, implizit der Totzeit der Strecke.

Obwohl die Drehzahlabweichungen beim Hochlaufen des Motors beim LMPC-Verfahren in der Simulation weitaus geringer sind, verschwindet der Drehzahlfehler nicht vollständig. Der Prädiktionshorizont des LMPC ist in der Simulation mit einem vorausschauenden Zeitintervall von $T_{P}=0.1 \mathrm{~s}$ voreingestellt. Allerdings werden in der Simulation für den konkreten Fall noch konstante Regelgrößen vorgegeben. Ein klassisches Signal-Previewing liegt nicht vor. Beim Signal-Previewing werden aktuelle und in der Zukunft sich zeitlich veränderte Referenzgrößen dem fortschreitenden Prädiktionshorizont übergeben und können so mit in die Optimierungsberechnung einbezogen werden. Dadurch wird es sicher möglich, den Regelfehler noch stärker zu reduzieren und die Regeleigenschaften zu verbessern. Der Regelfehler des PIDKaskadenreglers ist neben der Reglercharakteristik selbst auch der Verwendung des Führungsgrößenfilters und der Messwertfilter geschuldet. 
Die Abbildungen 6.10 und 6.11 zeigen das simulierte Verhalten des LMPC-Algorithmus im Vergleich zur PID-Regelung bei Aufschalten eine Störgröße, in der Simulation hier für ein sprungförmiges Lastmoment. Auch hier sind wie in den vorgehenden Abbildungen neben der Laständerung auch der Drehzahlverlauf, der Drehzahlfehler und die Stellgrößen aufgezeichnet. In Abbildung 6.10 wird die PMSM bei einer konstanten Drehzahl von $n=1000 \mathrm{U} / \mathrm{min}$ betrieben, in Abbildung 6.11 liegt der stationäre Arbeitspunkt bei $n=3500 \mathrm{U} / \mathrm{min}$.
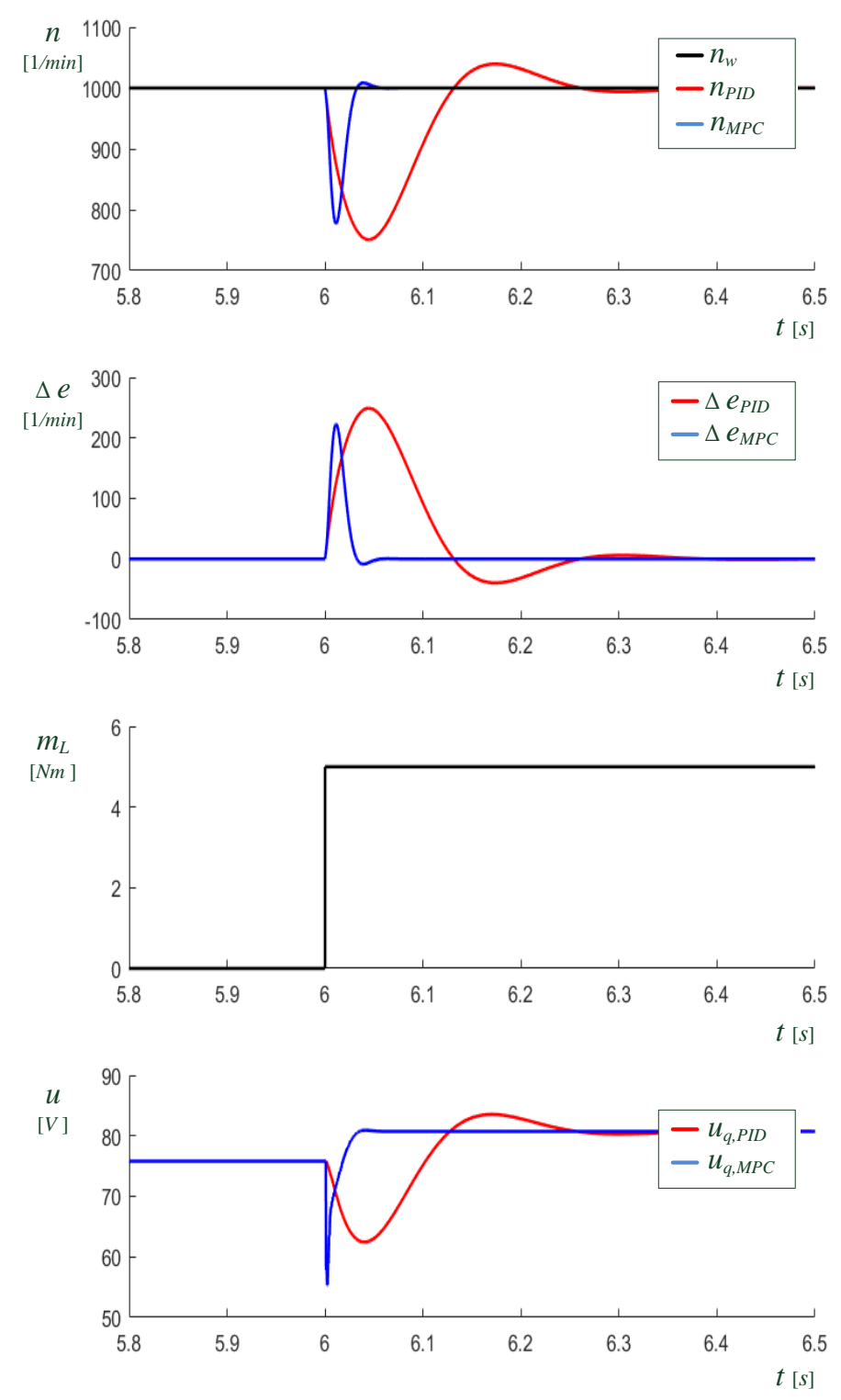

Abbildung 6.10.: Vergleich Störverhalten LMPC/PID für kleine Drehzahlen.

Zum Zeitpunkt $t=6 \mathrm{~s}$ wird für beide Drehzahlbereiche ein sprungförmiges Lastmo- 

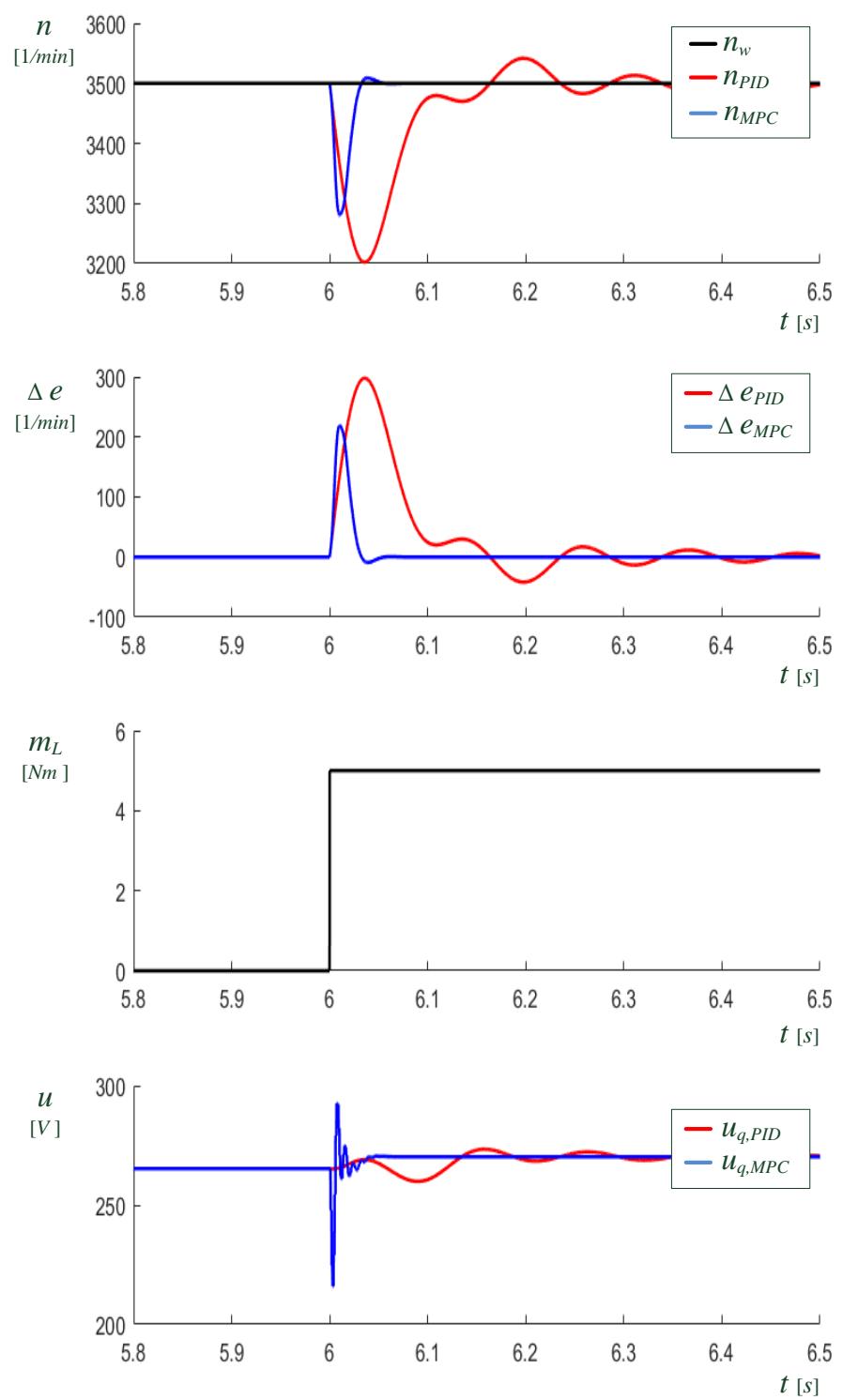

Abbildung 6.11.: Vergleich Störverhalten LMPC/PID für große Drehzahlen.

ment von $m_{L}=5 \mathrm{Nm}$ aufgeschaltet. In beiden Fällen kann der LMPC schneller auf den Lastsprung reagieren und den Drehzahleinbruch effektiver ausgleichen. Anders als der PID-Regler hat der LMPC durch seine prädiktive Arbeitsweise die Fähigkeit zu erkennen, dass keine Änderung der Führungsgröße vorgenommen wurde, sondern eine Störung vorliegen muss.

Während beim PID-Regler ein Kompromiss zwischen gutem Stör- und Führungsverhalten getroffen werden muss, kann der LMPC-Algorithmus gut auf Führungsgrößenverhalten und auf den Einfluss von Störgröße parametriert werden. Wie bereits in 
Abbildung 6.9 zu sehen ist, zeigt der PID-Regler auch in Abbildung 6.11 bei höheren Drehzahlen eine größere Drehzahlwelligkeit bzw. einen längeren Einschwingvorgang als bei der LMPC-Regelung.

\subsection{Zusammenfassung der Ergebnisse}

Mit der durchgeführten Simulation wurde gezeigt, dass es durch den Einsatz prädiktiver Regelungsalgorithmen möglich ist, bessere Ergebnisse für das Führungsverhalten und das Störverhalten einer PMSM zu erreichen als bei klassischen Regelverfahren. Natürlich steht außer Frage, dass noch geprüft werden muss, ob die getroffenen Vereinfachungen bei der Simulation, hinsichtlich der Linearisierung des MPC-ReglerModells, im realen Betrieb vergleichbare Ergebnisse erzielen.

Zu Beginn von Kapitel 6 werden die grundlegende Funktionsweise des modellprädiktiven Regelverfahrens und zwei bekannte Verfahren, das Dynamic Matrix Control Verfahren und das Generalized Predictive Control Verfahren, vorgestellt. Die Modellbildung der PMSM im statorfesten und rotorfesten Koordinatensystem wurde dargestellt, was die Basis für den späteren LMPC-Reglerentwurf in der Zustandsraumdarstellung bildete. Die MPC-Toolbox und der LMPC-Algorithmus wurden beschrieben und die Parameter des modellprädiktiven Regelverfahrens erläutert.

Wie sich bei den Simulationsergebnissen in Abschnitt 6.3.4 zeigte, weist die LMPCRegelung hinsichtlich der Regeleigenschaften ein besseres Führungsverhalten und Störverhalten aus als die klassische PID-Regelung. Bei der Untersuchung des Regelverhaltens für unterschiedliche Drehzahlbereiche bzw. Arbeitspunkte des Motors zeigten sich geringere Drehzahlwelligkeiten bei den Lastsprüngen und kürzere Einschwingvorgänge beim Führungsverhalten.

Prädiktive Regelungen besitzen die Fähigkeit, zukünftige Sollwertverläufe in die Prädiktion mit einzubinden. Gleichzeitig verwendet das modellprädiktive Regelverfahren ein Prozessmodell zur Berechnung der optimalen Steuergrößen anhand bisheriger Mess- und Zustandswerte und zukünftiger prädiktiver Werte der Regelgrößen. Durch die Einbindung der Sollwerttrajektorie kann das Führungsverhalten gezielt beeinflusst werden und ermöglicht eine frühzeitige Reaktion des geregelten Systems.

Die Verwendung von zukünftigen Sollwerten ist eine typische Eigenschaft prädiktiver Regelungen. In Verbindung mit einer im Hintergrund ablaufenden Zeitachse, die z.B. durch ein Zeitstempelverfahren definiert sein kann (vgl. Kapitel 4), können sich sämtliche Kommunikationsteilnehmer auf einer gemeinsamen Zeitachse synchronisieren. 
Eine dynamisch übergebene oder im Vorfeld hinterlegte Verfahrtrajektorie kann dabei im Sinne einer zeitgetakteten Sollwertvorgabe die Bewegungsabläufe untereinander kontrollieren und definieren. Start- und Stopp-Punkte sowie die dynamischen Bewegungsabläufe untereinander, ähnlich wie bei einer elektronischen Kurvenscheibe, können hinterlegt werden und die verschiedenen Antriebe können so einfach nach dem Prinzip einer virtuellen Steuerwelle miteinander synchronisiert werden.

Gegenüber einem klassischen Mikrocontroller kann hier eine SoPC-Plattform (vgl. Abschnitt 5.3.5) ein geeignetes Rechnersystem für einen MPC-Regler sein. Die Konfigurationsdaten können über den eingebundenen Soft-Core-Mikroprozesssor eingelesen bzw. parametriert werden.

In einem nächsten Schritt ist es möglich, das erarbeitete prädiktive Regelungskonzept in den Aufbau des realisierten Aktors in Abbildung $2.2 \mathrm{zu}$ integrieren. Hier kann die prädiktive Regelung innerhalb des intelligenten Aktors in dem mit grün schraffierten Bereich in Abbildung 2.2 eingebunden werden.

Die programmierbare Logik im FPGA ermöglicht die Bearbeitung paralleler Algorithmen und dadurch die Berechnung der aufwändigen Prädiktions- und Optimierungsaufwände in parallelen Zweigen. Innerhalb eines SoPC-basierten Embedded Systems ist es so möglich, ein kompaktes Aktor- und Kommunikationsmodul im Bereich der mobilen Intralogistik und beweglicher elektrischer Verbraucher zu realisieren. 



\section{Zusammenfassung}

In der vorliegenden Arbeit wird ein neues Konzept für einen intelligenten Aktor im Bereich der berührungslosen induktiven Energieübertragung bzw. beweglicher elektrischer Verbraucher vorgestellt.

Die Arbeit umfasst folgende inhaltliche Schwerpunkte:

- Übersicht über den derzeitigen Entwicklungsstand der berührungslosen Energieund Datenübertragung. Einordnung von Aktoren, intelligenten Aktoren und mechatronischen Systemen in das technische Umfeld (Kapitel 1).

- Vorstellung eines neuen Konzeptes für einen intelligenten Aktor im Bereich der induktiven kontaktlosen Energieübertragung für bewegliche Verbraucher. Darstellung der umzusetzenden unterschiedlichen funktionellen Komponenten für die Kommunikation, Datensynchronisierung und Regelung (Kapitel 2).

- Entwurf, Konstruktion und Aufbau eines induktiven kontaktlosen Energieübertragungssystems, im speziellen Fall für die Applikation eines neuartigen rotativen Prozessablaufes. Der Versuchsaufbau dient u.a. für die Validierung des Datenübertragungskonzeptes in Kapitel 4. Dabei wird untersucht, welche Erkenntnisse sich aus dem Versuchsaufbau ergeben, im Besonderen für das Übertragungsverhalten des Datenkanals, der über die induktive kontaktlose Energieversorgung ermöglicht werden soll (Kapitel 3).

- Untersuchung und Realisierung wie eine Datenkommunikation und eine Synchronisation zwischen stationären und beweglichen Kommunikationsteilnehmern am Versuchsaufbau realisiert werden können, die über das induktive kontaktlose Energieübertragungssystem miteinander verbunden sind (Kapitel 4).

- Umsetzung des entwickelten Konzeptes für eine neue Hardware- und Softwareplattform für einen intelligenten kompakten Aktor im Bereich einer SoPCbasierten Embedded Systemplattform (Kapitel 5).

- Realisierung eines prädiktiven Regelungskonzeptes für einen intelligenten Aktor im Bereich der beweglichen elektrischen Verbraucher, wenn Daten über den stark störungsbehafteten und bandbegrenzten Datenkanal des induktiven kontaktlosen Energieübertragungssystems übertragen werden. (Kapitel 6). 
Die wichtigsten Ergebnisse der Arbeit sind:

1. Konstruktion und Aufbau eines Funktionsmusters für eine rotative Prozessbewegung mit Einbindung eines induktiven kontaktlosen Energieübertragungssystems. Dabei wurden:

- am realisierten Versuchsaufbau gezeigt, wie ein induktives kontaktloses Energieübertragungssystem in den Versuchsaufbau integriert und wie die Linienleiterführung für die Energiezuführung für ein solches System aufgebaut werden kann,

- die Eigenschaften und die Störeinflüsse des Energieübertragungssystems als Datenübertragungskanal analysiert und beschrieben,

- die Übertragungseigenschaften bzw. der optimal nutzbare Frequenzbereich für die Datenübertragung bestimmt,

- die Parameter des Datenübertragungssystems entsprechend den Eigenschaften und den Störeinflüssen des Datenübertragungskanals ermittelt.

2. Nachweis, dass es durch die Wahl eines geeigneten Übertragungs- und Modulationsverfahren möglich ist, eine Kommunikation und Synchronisation über den kontaktlosen Energieübertragungskanal zu realisieren. Dabei war es möglich:

- eine kontaktlose Datenübertragung am realisierten Versuchsaufbau für gewählte Trägerfrequenzen über den stark gestörten Datenkanal zu integrieren,

- durch parallele Abarbeitungsalgorithmen im FPGA die Durchlaufzeiten der Signale im Sende- und Empfangspfad des Modems innerhalb der OFDMbasierten Datenkommunikation zu bestimmen,

- über das entwickelte integrierte Zeitstempelverfahren die Kommunikationsteilnehmer über den stark störungsbehafteten induktiven kontaktlosen Energieübertragungskanal miteinander zu synchronisieren,

- zu zeigen, dass am Versuchsaufbau die Synchronisationsgenauigkeit zwischen Master- und Slaveuhr bzw. zwischen zwei Kommunikationsteilnehmern innerhalb eines Zeitfensters von wenigen $\mu s$ erreicht wird.

3. Untersuchung und Analyse der Ganggenauigkeit bzw. der Frequenzkonstanz von verschiedenen Oszillatorklassen. Dabei konnte gezeigt werden:

- die qualitative Bewertung der Ganggenauigkeit von Oszillatoren bei der Betrachtung gleicher Oszillatorklassen durch die Zwei-Oszillator-Methode, 
- dass sich durch den Einsatz ausgewählter höherwertiger Oszillatoren die Taktfrequenz für die Synchronisierungsvorgänge bei der Übertragung von Daten zwischen einzelnen Kommunikationsteilnehmern deutlich reduzieren lässt,

- die Anwendung eines Verfahrens zur Bestimmung der Frequenzkonstanz unterschiedlicher Oszillatorklassen auf Basis der Allan-Varianz.

4. Entwurf und Entwicklung einer Hardware- und Regelungsplattform für einen intelligenten Aktor im Bereich der beweglichen elektrischen Verbraucher. Folgende Punkte wurden gezeigt bzw. erarbeitet:

- die Darstellung und der Entwurf für ein kompaktes Motorsteuerkonzept innerhalb eines SoPC-basierten Embedded Systems, alternativ zum Entwurf eines klassischen Standard-Mikrocontroller Layouts,

- der Aufbau eines skalierbaren und modularisierbaren Motorsteuerkonzeptes für den Betrieb einer PMSM durch die Einbindung programmierbarer Hardware und Soft-Cores sowie peripheren IP-Blöcken, mit der Möglichkeit zusätzliche benötigte Strukturen für weitere Aktoren oder Funktionen in die FPGA-Plattform einzubinden,

- die Realisierung der Regelungsmodule und der Module für die Koordinatentransformationen innerhalb der Regelungs- und Steuerungsplattform effizient in VHDL-Code, Custom Instructions und C-Code,

- die Integration und Auswertung der wichtigen Motorstrom-Phasenmessung durch die Verwendung von $\Sigma \Delta$-Wandler im FPGA-Konzept. Dabei wurde die Phasenstrommessung in Kombination mit FPGA-basierter Technik gegenüber den bekannten Strommessverfahren u.a. durch Nutzung der digitalen Filterung im FPGA vereinfacht und verbessert,

- die Bestimmung der Phasenstrommessung für unterschiedliche Genauigkeitsanforderungen und Reaktionszeiten innerhalb der FPGA-basierten Architektur.

5. Entwicklung und Umsetzung eines prädiktiven Regelungsverfahrens im Konzept der beweglichen elektrischen Verbraucher. Der Schwerpunkt der Betrachtung lag auf:

- der Beschreibung der Modellbildung der Regler und Regelstrecke für den Betrieb einer PMSM für eine prädiktive und eine klassische regelungstechnische Betrachtung in der durchgeführten Simulation,

- dem Entwurf des linearisierten Modells der PMSM in der Zustandsraumdarstellung für den Reglerentwurf des MPC-Reglers, 
- der Einbindung und Berechnung der optimalen Steuergrößen anhand bisheriger Mess- und Zustandswerte und zukünftiger prädiktiver Werte der Regelgröße sowie der Bestimmung der Reglerparameter der prädiktiven LPMC-Regelung,

- dem Nachweis der Leistungsfähigkeit der MPC-Regelung im Vergleich zu einer klassischen PID-Kaskadenregelung in der durchgeführten Simulation und der Darstellung des Stör- und Führungsverhaltens des LMPCAlgorithmus alternativ zur klassischen PID-Regelung für den Betrieb einer PMSM.

Weiterführende Aufgaben im Konzept des intelligenten Aktors sind die Zusammenführung der Hardwareplattform für den Steuerteil des Umrichters mit der Kommunikations- und Regelungsplattform sowie die Einbindung der Soft-Core-CPU in das Gesamtkonzept.

Im Bereich des Kommunikationsprotokolls können Optimierungen hinsichtlich der Integration einer fortlaufenden Sendezyklusnummer im Datentelegramm und der Plausibilitätskontrolle des Zeitstempels realisiert werden. Zur Verbesserung der Synchronisationsgenauigkeit ist die Ermittlung des genauen Erkennungszeitpunktes des empfangenen Chirpsignales im Empfangspfad zu bestimmen um die Blockbearbeitung des FFT/IFFT-Blocks exakt darauf zu synchronisieren. Die externe Datenanbindung der SSC-Schnittstelle zum FPGA kann durch die eingebundene Soft-Core-CPU ermöglicht werden.

Ein weiterer Schritt ist die Umsetzung der LMPC-Regelung auf die reale FPGAHardwareplattform. Dabei ist zu prüfen, ob die getroffenen Vereinfachungen bei der Simulation hinsichtlich der Linearisierung des MPC-Regler-Modells im realen Betrieb ein vergleichbares prädiktives Verhalten erzielen.

Ein wichtiger Bestandteil ist die Einbindung der Optimierungsverfahren innerhalb der FGPG-basierten Umgebung. Die Realisierung der modellbasierten Regelung im FPGA ermöglicht die Bearbeitung paralleler Algorithmen und die Berechnung der aufwändigen Prädiktions- und Optimierungsaufwände in parallelen Zweigen. Beschränkungen von Stellgrößen, Stellgrößenänderungen und Ausgangsgrößen sind als Teil der Optimierung zu berücksichtigen, wobei die Randbedingungen als Soft-Constraints oder auch als exakt zu erfüllende Hard-Constraints eingebunden werden können.

Durch Einbindung des Signal-Previewing können aktuelle und in der Zukunft sich zeitlich veränderte Referenzgrößen dem fortschreitenden Prädiktionshorizont übergeben und so in die Optimierungsberechnung mit einbezogen werden. 
Innerhalb der Umgebung lokaler hochgenauer Uhren auf Basis einer ablaufenden Zeitachse können vernetzte Teilnehmer im Sinne einer zeitgetakteten Sollwertvorgabe einfach miteinander synchronisiert werden. Durch die Einbindung zukünftiger bekannter Sollwertverläufe im Regelungsverfahren ist das Führungsverhalten gezielt beeinflussbar und ermöglicht eine frühzeitige Reaktion des geregelten Systems auf Lastsprünge oder sonstigen Störungen. 



\section{Literaturverzeichnis}

[1] 3 eQ: Intelligente Aktoren. https://www.eq-3.de/partner/oem.html. - Eingesehen am 15.02.2020

[2] Agbinya, J.: Wireless Power Transfer. Burleson : River Publisher, 2012

[3] Allan, D.: The Allan Varianz. http://www.allanstime.com/ AllanVariance/. - Eingesehen am 18.01.2020

[4] Altera Corporation: Simultaneous Multi-Mastering with the Nios Processor. https://www.intel.co.jp/content/dam/altera-www/global/ja_JP/ pdfs/literature/tt/tt_nios_smm.pdf. Version:2002. - Eingesehen am 25.02.2017

[5] Altera Corporation: Cyclone III device handbook. https: //www.intel.com/content/dam/altera-www/global/en_US/pdfs/ literature/hb/cyc3/cyclone3_handbook.pdf. Version: August 2012. Eingesehen am 25.02.2017

[6] Altera Corporation: Quartus II Software. http://www.altera.com/ support/software/sof-quartus.html. Version:2013. - Eingesehen am 24.02.2018

[7] Altera Corporation: Nios II Custom Instructions User Guide. https://www.intel.com/content/dam/altera-www/global/en_US/pdfs/ literature/ug/archives/ug-nios2-custom-instruction-15.1.pdf. Version: 2015. - Eingesehen am 17.02.2018

[8] Altera Corporation: Nios II Classic Processor Reference Guide. https://www.intel.com/content/dam/www/programmable/us/en/pdfs/ literature/hb/nios2/n2cpu_nii5v1.pdf. Version:2016. - Eingesehen am 13.10.2018

[9] Altera Corporation: Avalon Interface Specifications. https://www.intel.com/content/dam/altera-www/ global/en_US/pdfs/literature/manual/archives/mnl_avalon_ spec-quartus-prime-pro-v17-1-stratix-10-es-editions.pdf. Version: 2017. - Eingesehen am 24.02.2018

[10] Altera Corporation: Datenblatt: EPCS64 Configuration Device. https://www.intel.com/content/dam/www/programmable/us/en/pdfs/ 
literature/hb/cfg/cyc_c51014.pdf. Version: April 2014. - Eingesehen am 08.04.2018

[11] Andelfinger, V. P. ; HÄNisch, T.: Industrie 4.0 : Wie cyber-physische Systeme die Arbeitswelt verändern. Wiesbaden : Springer Gabler Verlag, 2017

[12] Arato, L. ; Graf, U.: Einführung in ModelSim. NTB, Interstaatliche Hochschule für Technik Buchs. https://wiki.ntb.ch/infoportal/_media/ software/modelsim/einfuehrung_in_modelsim_v0.9.pdf. Version:2014. Eingesehen am 05.03.2017

[13] ARM COMPANY: AMBA Specifcation. ARM. https://www. arm.com/products/silicon-ip-system/embedded-system-design/ amba-specifications. Version:2017. - 2. Auflage, Eingesehen am 18.02 .2018

[14] Arzet, H.: Grundlagen des One Piece Flow. Berlin : Rhombos Verlag, 2005

[15] Asfour, T.: Humanoide Roboter auf dem Vormarsch. Karlsruhe : KIT-Bibliothek Süd, 2017 https://mediaservice.bibliothek.kit.edu/\#/ details/DIVA-2017-108/0. - Online-Ressource (Film), eingesehen am 16.05.2020

[16] Avago Technologies: Datenblatt: $A C P L-4800-300$ https://docs . broadcom.com/doc/AV01-0193EN. Version: 2007. - Eingesehen am 25.11.2018

[17] Avago Technologies: Datenblatt: HCPL-796J. https://docs.broadcom. com/doc/AV02-1670EN. Version: 2016. - Eingesehen am 14.10.2018

[18] Ballas, R. ; Pfeiffer, G. ; R., Werthschützky.: Elektromechanische Systeme in Mikrotechnik und Mechatronik. Berlin : Springer Verlag, 2009. - 2. Auflage

[19] Bandemer, H. ; Gottwald, S.: Einführung in Fuzzy-Methoden: Theorie und Anwendungen unscharfer Mengen. Berlin : Akademie-Verlag, 1992. - 3. unveränderte Auflage

[20] Bauch, J. ; Hoppe, A. ; Nesterov, M.: Sichere kontaktlose induktive Datenübertragung für interoperable mobile Systeme - Vorteile und Anwendungsmöglichkeiten. Baden-Baden : AUTOMATION 2018, Seamless Convergence of Automation \& IT, 03.-04.07.2018, VDI Verlag, 2018. - S. 249-260

[21] BEIs, U.: Eine Einführung in Delta-Sigma-Wandler. https://www.beis.de/ Elektronik/DeltaSigma/DeltaSigma_D.html. Version:2010. - Eingesehen am 20.04.2019

[22] Bemporad, A. ; Morari, M. ; Ricker, N.L.: Model Predictive Control Toolbox User's Guide. R2017a. 3 Apple Hill Drive Natick, MA 01760-2098: The MathWorks, Inc., March 2017

[23] Bergold, S. ; Ebersberger, H.: Eine kompakte Darstellung der Struktur modellprädiktiver Regelungsverfahren für Mehrgrößensysteme. In: atAutomatisierungstechnik 46 (1998), Nr. 10, S. 468 
[24] Bertsche, B. ; Göhner, P. ; Jensen, U. ; Schinköthe, W. ; Wunderlich, H. J.: Zuverlässigkeit mechatronischer Systeme: Grundlagen und Bewertung in frühen Entwicklungsphasen. Berlin : Springer Verlag, 2009

[25] Beuth, K. ; Breide, S. ; Lüders, C.F. ; Kurz, G. ; Hanebuth, R.: Nachrichtentechnik. Würzburg : Vogel Business Media, 2015. - 4. Auflage

[26] Beyerer, J. ; Jasperneite, J. ; Sauer, O.: Industrie 4.0. In: atAutomatisierungstechnik 63 (2015), Nr. 10, S. 751-752

[27] Braun, P. ; Grüne, L.: Verteilte Optimierung: Anwendungen in der Modellprädiktiven Regelung. In: at-Automatisierungstechnik 66 (2018), Nr. 11, S. 939-949

[28] Bräunl, T.: Embedded Robotics: Mobile Robot Design and Applications with Embedded Systems. Berlin : Springer Verlag, 2008. - 3. Auflage

[29] Bullinger, H.-J. ; Ten Hompel, M.: Internet der Dinge. Berlin : Springer Verlag, 2007

[30] Burrus, C.S.: Gauss and the History of the Fast Fourier-Transform. In: Archive for History of Exact Sciences 34 (1985), Nr. 03, S. 265-277

[31] Busch, R.: Elektrotechnik und Elektronik. Berlin : Springer Verlag, 2015

[32] Buxbaum, H. J.: Mensch-Roboter-Kollaboration. Wiesbaden : Springer Gabler Verlag, 2020

[33] Camacho, E.F. ; Bordons, C.: Model Predictive Control. London : Springer Verlag, 1999

[34] Camacho, E.F. ; Bordons, C.: Model Predictive Control. London : Springer Verlag, 2007 (Advanced textbooks in control and signal processing). - 2. ed.

[35] Candy, J.C: Decimation for Sigma Delta Modulation. In: IEEE Trans. Commun. (1986), Nr. 34, S. 72-76

[36] Carcia, C. ; Prett, D. ; Morari, M.: Model Predictive Control: Theory and Practice - a Survey. In: Automatica 25 (1989), Nr. 03, S. 335-348

[37] Clarke, D. ; Mohtadi, C. ; Tuffs, P.: Generalized Predictive Control Part II. Extensions and Interpretations. In: Automatica 23 (1987), Nr. 02, S. 149-160

[38] Clarke, D. ; Mohtadi, C. ; Tuffs, P.: Generalized Predictive Control - Part II. The Basic Algorithm. In: Automatica 23 (1987), Nr. 02, S. 137-148

[39] Conductix-Wampfler GmbH: Inductive Power Transfer IPT. https:// www.conductix.de/de. - Eingesehen zuletzt am 22.02.2020

[40] Contadini, F.: Entzaubert, Delta-Sigma AD-Wandler, Teil 1. In: elektronik industrie 04 (2002), Nr. 05, S. 20-23

[41] Cooley, J.W. ; Tukey, J.W.: An algorithm for the machine calculation of complex Fourier series. In: Mathematics of Computation 19 (1965), S. 297-301 
[42] Cravero, L.: Entwurf Auslegung und Betriebsverhalten von dauermagneterregten bürstenlosen Motoren kleiner Leistung, Technische Universität Ilmenau, Diss., 2006

[43] Czichos, H.: Mechatronik - Grundlagen und Anwendungen technischer Systeme. Wiesbaden : Springer Vieweg Verlag, 2019. - 4., überarbeitete und erweiterte Auflage

[44] Deppe, J.H. ; Trsek, H. ; Jasperneite, J.: WLAN-Geräte für die Industrie im Vergleich. In: Elektronik (2011), Nr. 06, S. 42-50

[45] Dittmar, R. ; Pfeiffer, B.M.: Modellbasierte prädiktive Regelung - eine Einführung für Ingenieure. München : Oldenbourg Wissenschaftsverlag, 2004

[46] Dostert, K.: Powerline Kommunikation. Poing : Franzis Verlag, 2000

[47] Dostert, K.: Powerline Kommunikation: Smart HomeGebäudeautomatisierung, Internet aus der Steckdose, EMV-Aspekte. Poing : Franzis Verlag, 2000

[48] EBV Elektronik: Datasheet DBC3C40 Cyclone III Development Board. https://www.devboards.de/startseite/produkte/produkte-details/ article/dbc3c40/. Version:2014. - Eingesehen am 14.10.2017

[49] EBV EleKtronik: Falconeye. https://www.devboards.de/startseite/ produkte/produkte-details/article/falconeye-fpga/. Version:2014. Eingesehen am 07.01.2018

[50] EssER, A.: Berührungslose, kombinierte Energie- und Informationsübertragung für bewegliche Systeme, RWTH Aachen, Diss., 1992

[51] Eichin, D.: Modellbasiertes Konzept zur vollautomatisierten Montageendprüfung von asynchron angetriebenen Getriebemotoren im lastlosen Zustand, Karlsruher Institut für Technologie (KIT), Diss., 2015

[52] EIDAM, J.: Beurteilung und Simulation des Betriebsverhaltens von lagegeregelten elektrischen Direktantrieben als „Elektronische Kurvenscheibe“. Düsseldorf : VDI-Verlag, 1997

[53] Eidson, J.: Measurement, Control, and Communication Using IEEE 1588. London : Springer Verlag, 2006

[54] Eigner, M. ; Gerhardt, F. ; Gilz, T. ; Nem, F.M.: Informationstechnologie für Ingenieure. Berlin : Springer Verlag, 2012

[55] Elsing, J.: Das OSI-Schichtenmodell : Grundlagen und Anwendungen der X.200. Vaterstetten bei München : IWT-Verlag, 1991

[56] Engels, H.: CAN-Bus: Feldbusse im Überblick, CAN-Bus-Protokolle, CANBus-Meßtechnik, Anwendungen. Poing : Franzis Verlag, 2002. - 2., überarbeitete Auflage

[57] Engemann, C. ; Sudmann, A.: Machine Learning: Medien, Infrastrukturen und Technologien der Künstlichen Intelligenz. Bielefeld : transcript Verlag, 
2018

[58] Fairchild Semiconductor: Datenblatt: FSQ321. https://www.onsemi. com/pub/Collateral/FSQ321-D.pdf. Version:2011. - Eingesehen am 10.03.2018

[59] Fairchild Semiconductor: Datenblatt: FSBB15CH60. https://www. onsemi.com/pub/Collateral/FSBB15CH60-D.pdf. Version:2014. - Eingesehen am 11.03.2017

[60] Fazlollahtabar, H. ; SAidi-Mehrabad, M.: Autonomous Guided Vehicles : Methods and Models for Optimal Path Planning. Cham : Springer International Publishing, 2015

[61] Fletcher, R.: Practical Methods of Optimization. Chichester, UK : John Wiley, 1986

[62] FlüGEL, H.: FPGA-Design mit Verilog. Oldenbourg Wissenschaftsverlag $\mathrm{GmbH}, 2010$

[63] Föllinger, O.: Regelungstechnik - Einführung in die Methoden und ihre Anwendungen. Berlin : VDE Verlag, 2016. - 12. überarbeitete Auflage

[64] Gerke, W.: Technische Assistenzsysteme: vom Industrieroboter zum Roboterassistenten. Berlin : De Gruyter Oldenbourg Verlag, 2015

[65] GöHner, P.: Agentensysteme in der Automatisierungstechnik. Berlin : Springer Verlag, 2013

[66] Günter, U. ; Albrecht, T.: Fahrerlose Transportsysteme: Eine Fibel - mit Praxisanwendungen - zur Technik - für die Planung. Wiesbaden : Springer Vieweg Verlag, 2019. - 3. Auflage

[67] Goebel, G. ; Allgöwer, F.: New results on semi-explicit and almost explicit MPC algorithmus. In: at-Automatisierungstechnik 65 (2017), Nr. 04, S. 245-251

[68] Grüne, L. ; Pannek, J.: Nonlinear Model Predictive Control - Theory and Algorithms. London : Springer Verlag, 2017. - 2. Auflage

[69] Gruhler, G.: Feldbusse und Geräte-Kommunikationssysteme: praktisches Know-How mit Vergleichsmöglichkeiten; ARCNET, AS-Interface, BITBUS, CAN, FIP, LON, Industrial Ethernet, Interbus, Profibus-FMS, SicherheitsBussysteme, Vergleichslisten. Poing : Franzis Verlag, 2001

[70] ÖGÜT, B.: Untersuchung der Positionsabhängigkeit eines Übertragungskanals in einem kontaktlosen Energie- und Datenübertragungssystem, Karlsruher Institut für Technologie (KIT), Institut für Technik der Informationsverarbeitung (ITIV), Masterarbeit, 2010

[71] Heidenhain GmbH: Documentation Endat. http://www.heidenhain. de/de_DE/dokumentation/grundlagen/schnittstellen/EnDat-22.

Version: 2015. - Eingesehen am 25.02.2017

[72] Henning, J. ; Künzel, G.: Flaschen-Etikettiermaschinen im Überblick. In: 
Neue Verpackung 59 (2006), Nr. 07, S. 28-32

[73] Hering, E. ; Bressler, K. ; Gutekunst, J.: Elektronik für Ingenieure und Naturwissenschaftler. Berlin : Springer Vieweg Verlag, 2017. - 7. Auflage

[74] Hering, E. ; Martin, R. ; Stohrer, M.: Physik für Ingenieure. Berlin : Springer Verlag, 2016. - 12. Auflage

[75] Hertzberg, J. ; Lingemann, K. ; Nüchter, A.: Mobile Roboter: eine Einführung aus Sicht der Informatik. Berlin : Springer Vieweg Verlag, 2012

[76] Herveille, R.: WISHBONE System-on-Chip (SoC) Interconnect Architecture for Portable IP Cores. OpenCores. http://www.opencores.org. Version: 2004. - b.3 Auflage, Eingesehen am 18.02.2019

[77] Hewlett-PaCkard Company (Hrsg.): Fundamentals of Quartz Oscillators, Application Note. 200-2. United States: Test and Measurement Call Center, P.O. Box 4026, Englewood, CO 80155-4026: Hewlett-Packard Company, Mai 1997

[78] Hofmann, J.: Die digitale Fabrik: auf dem Weg zur digitalen Produktion. Berlin : VDE Verlag, 2017

[79] Hoppe, A.: Neue Einsatzmöglichkeiten und Entwicklungstendenzen in der kontaktlosen Energie- und Datenübertragung. In: ETG journal (2014), Nr. 02, S. $61-63$

[80] HuA, Z.: Ein robustes OFDM-Systemkonzept zur schnellen Datenübertragung in der kontaktlosen Energieversorgungstechnik, Universität Karlsruhe (TH), Diss., 2007

[81] IBM Corporation: PPC440 Core Users Manual. http://class.ece. iastate.edu/cpre584/ref/Xilinx/edk/ppc440x5_um.pdf. Version: 2003. Eingesehen am 11.02.2017

[82] IBM Corporation: CoreConnect Bus Architecture. https://web. archive.org/web/20090211095135/http://www-01.ibm.com/chips/ techlib/techlib.nsf/productfamilies/CoreConnect_Bus_Architecture. Version: 2007. - Eingesehen am 18.02.2017

[83] International Commission on Non-Ionizing Radiation Protection (ICNIRP): Guidelines for limiting exposure to time-varying electric and magnetic fields (1 Hz to $100 \mathrm{kHz}$ ). In: Health Physics 99 (2010), Nr. 06, S. $818-836$

[84] Isermann, R.: Mechatronische Systeme - Grundlagen. Berlin : Springer Verlag, 2008. - 2. Auflage

[85] JABER, A.A.: Design of an Intelligent Embedded System for Condition Monitoring of an Industrial Robot. Cham : Springer Verlag, 2017

[86] Janocha, H.: Aktoren: Grundlagen und Anwendungen. Berlin : Springer Verlag, 1992 
[87] Janocha, H.: Unkonventionelle Aktoren: Eine Einführung. München : Oldenbourg Verlag, 2013. - 2., ergänzte und aktualisierte Auflage

[88] JanscheK, J.: Systementwurf mechatronischer Systeme: Methoden - Modelle - Konzepte. Berlin : Springer Verlag, 2010

[89] Janssen, D.: EtherCat als Antriebsbus - Synchronität ist nicht genug. In: IEEE 49 (2004), Nr. 11, S. 18-22

[90] Jazdi-Motlagh, N. ; Marks, P. ; Aivaliotis, A. ; Weyrich, M.: Ein agentenbasiertes Assistenzsystem zur dynamischen Zuverlässigkeitsberechnung von cyber-physischen Systemen. In: at-Automatisierungstechnik 65 (2017), Nr. 11, S. 779-792

[91] Jenni, F. ; WüEst, D.: Steuerverfahren für selbstgeführte Stromrichter. Stuttgart : Teubner Verlag, 1995

[92] Jondral, F.: Nachrichtensysteme Grundlagen-Verfahren-Anwendungen. Weil der Stadt : J. Schlembach Fachverlag, 2001

[93] Jung, H.: Konzept einer agentenbasierten Transportsteuerung für komplexe, dynamische und multimodale Logistiknetzwerke, Karlsruher Institut für Technologie (KIT), Diss., 2015

[94] KafkA, G.: WLAN: Technik, Standards, Plannung und Sicherheit für Wireless LAN. München : Hanser Verlag, 2005

[95] Kammeyer, K.: Nachrichtenübertragung. Wiesbaden : Verlag Vieweg + Teubner, 2011. - 4., neu bearbeitete und ergänzte Auflage

[96] Karrenberger, U.: Signale - Prozesse - Systeme. Berlin : Springer Vieweg Verlag, 2017. - 7., neu bearbeitete und erweiterte Auflage

[97] Kaufmann, T. ; Servatius, H. G.: Das Internet der Dinge und Künstliche Intelligenz als Game Changer: Wege zu einem Management 4.0 und einer digitalen Architektur. Wiesbaden : Springer Vieweg Verlag, 2020

[98] Kegel, G.: Industry 4.0 - more than just the next generation of automation technology. In: at-Automatisierungstechnik 65 (2017), Nr. 10, S. 669-671

[99] Kesselring, J.: Prozessbegleitende Planung und Konfiguration von Fördertechnikanlagen unter Zuhilfenahme von virtuellen Konfigurationsmustern und Konfigurationsmodellen, Karlsruher Institut für Technologie (KIT), Diss., 2017

[100] Khadour, Y.: Eignung von Niederspannungsverteilnetzen für Powerline Communication, Universität Duisburg-Essen, Diss., 2003

[101] KIEL, E.: Antriebslösungen: Mechatronik für Produktion und Logistik. Berlin : Springer Verlag, 2007

[102] Kiendl, H.: Fuzzy Control methodenorientiert. Berlin : Oldenbourg Wissenschaftsverlag, 2018

[103] Kinnebrock, W.: Neuronale Netze: Grundlagen, Anwendungen, Beispiele. 
Berlin : Oldenbourg Wissenschaftsverlag, 2018. - 2., verbesserte Auflage

[104] KistneR, T.: Ein neuartiges mehrträgerbasiertes PLC-System mit störresistenter Synchronisation, Universität Karlsruhe (TH), Diss., 2008

[105] Klasen, F. ; Oestreich, V. ; Volz, M.: Industrielle Kommunikation mit Feldbus und Ethernet. Berlin : VDE Verlag, 2010

[106] Knaup, P.: Berührungslose Energieübertragung auf linear bewegte Systeme, Universität Darmstadt, Diss., 1999

[107] KÖnneKeR, C.: Roboter und künstliche Intelligenz. Heidelberg : Spektrum der Wissenschaft Verlagsgesellschaft mbH, 2014

[108] Kovacs, K.P. ; Racz, I.: Transiente Vorgänge in Wechselstrommaschinen. Band I. Budapest : Verlag der ungarischen Akademie der Wissenschaften, 1959

[109] Krah, J.O. ; Höltgen, M. ; Richter, R.: FPGA-basierte Motorsteuerung mit $\Sigma \Delta$-Strommessung. In: Elektronik 15 (2013), Nr. 07, S. 18-23

[110] Kraus, P.: Prädiktive Regelung mit linearen Prozeßmodellen im Zustandsraum, RWTH-Aachen, Diss., 1996

[111] Krischke, A.: Rothammels Antennenbuch. Baunatal : DARC Verlag, 2013.-13. Auflage

[112] Kroll, A.: Computational Intelligence: Probleme, Methoden und technische Anwendungen. Berlin : De Gruyter Oldenbourg, 2016. - 2. Auflage

[113] Kroll, A. ; Abel, D.: Modellbasierte Prädiktive Regelung - Model Predictive Control. In: at-Automatisierungstechnik 54 (2006), Nr. 12, S. 590-601

[114] Krones AG: Internet-Präsens Etikettiermaschinen. https://www.krones. com/de/produkte/maschinen/etikettiermaschinen.php. Version: Juni 2017. - Eingesehen am 08.12.2018

[115] KüRsChNeR, D.: Methodischer Entwurf toleranzbehafteter induktiver Energieübertragungssysteme. Aachen : Shaker Verlag, 2010

[116] Kruse, R. ; Borgelt, C. ; Braune, C. ; Klawonn, F. ; Möwes, C. ; Steinbrecher, M.: Computational Intelligence : Eine methodische Einführung in Künstliche Neuronale Netze, Evolutionäre Algorithmen, Fuzzy-Systeme und Bayes-Netze. Wiesbaden : Springer Vieweg Verlag, 2015. - 2. Auflage

[117] Kurzweil, P. ; Frenzel, B. ; Gebhard, F.: Physik - Formelsammlung für Ingenieure und Naturwissenschaftler. Wiesbaden : Friedr. Vieweg \& Sohn Verlag | GWV Fachverlage, 2008

[118] KövarI, L.: Konzeption und Realisierung eines neuen Systems zur produktbegleitenden virtuellen Inbetriebnahme komplexer Förderanlagen, Karlsruher Institut für Technologie (KIT), Diss., 2011

[119] Laschewski-Grossbaier, R. ; Mack, T.: Intelligent und sicher - Smarte 
Aktoren im Automobil. In: Elektronik-automotive 20 (2019), Nr. 02, S. 38-40

[120] Lee, J. ; Morari, M. ; CArcia, C.: State-Space Interpretation of Model Predictive Control. In: Automatica 30 (1994), Nr. 04, S. 707-717

[121] Lenze Vertrieb GmbH: Dezentrale Antriebssteuerungen. https://www. lenze.com/de-de/produkte. - Eingesehen zuletzt am 25.01.2020

[122] LEUER, M.: Modellprädiktive Regelung permanent erregter Synchronmotoren im Antriebsstrang von Automobilen, Universität Paderborn, Diss., 2015

[123] LEUZE ELECTRONIC: Datenübertragungslichtschranken. https: //www. leuze.com/de/deutschland/produkte/datenuebertragung_ steuerungskomponenten/datenuebertragung/index.php. Version:2020. Eingesehen am 11.04.2020

[124] Ling, K.V. ; Maciejowski, J.M. ; Richards, A.G. ; Wu, B.F.: Multiplexed Model Predictive Control. In: University of Cambridge, Department of Engineering, Technical Report Cued/F-Infeng/Tr.657 (2010)

[125] Lohse, M.: Nutzerfreundliche Mensch-Roboter-Interaktion: Kriterien für die Gestaltung von Personal Service Robots. Saarbrücken : VDM Verlag Dr. Müller, 2007

[126] Lundström, P. ; Lee, J. ; Morari, M. ; Skogestad, S.: Limitations of Dynamic Matrix Control. In: Computers and Chemical Engineering 19 (1995), Nr. 04, S. 409-421

[127] Lunze, J.: Regelungstechnik 1, Systemtheoretische Grundlagen, Analyse und Entwurf einschleifiger Regelungen. Berlin : Springer Vieweg Verlag, 2013. - 9., überarbeitete Auflage

[128] Maciejowski, J.M.: Predictive Control with Constraints. Harlow : Prentice Hall, 2008

[129] Mainzer, K.: Künstliche Intelligenz - Wann übernehmen die Maschinen. Berlin : Springer Verlag, 2019. - 2. Auflage

[130] Marwedel, P.: Embedded System Design: Embedded Systems, Foundations of Cyber-Physical Systems, and the Internet of Things. Cham : Springer Verlag, 2018. - 3. Auflage

[131] MathWorks: Documentation QP-Solver. htps://de.mathworks.com/help/ mpc/ug/qp-solver.html. Version: 2017. - Eingesehen am 28.04.2018

[132] MathWorks: Optimization Toolbox User Guide. https://de.mathworks. com/help/optim/. Version: 2017. - Eingesehen am 28.01.2018

[133] Maxim InTEgrated: Datenblatt: MAX3238 UART Transceiver. https://www.maximintegrated.com/en/products/interface/ transceivers/MAX3238.html. Version: 2002. - Eingesehen am 13.05.2018

[134] MaYeR, S.: Development of a completely decentralized control system for modular continuous conveyors, Karlsruher Institut für Technologie (KIT), Diss., 
2009

[135] Merz, H. ; Hansemann, T. ; HüBner, C.: Building Automation: Communication systems with EIB/KNX, LON and BACnet. Cham : Springer Verlag, 2018. - 2nd ed.

[136] MIKROCONTROLLER.NET: FPGA. http://www.mikrocontroller.net/ articles/FPGA. Version: 2014. - Eingesehen am 10.02.2018

[137] MIPS Technologies Inc.: MIPS Architecture. https://www.mips.com/ products/architectures/nanomips/. Version:2015. - Eingesehen am 23.06.2018

[138] Mishra, B.B. ; Dehuri, S. ; Panigrahi, B.K. ; NayaK, A. K. ; B.S.P., Mishra ; DAS, H.: Computational Intelligence in Sensor Networks. Berlin : Springer Verlag, 2019

[139] Mohamed, K.S.: IP Cores Design from Specifications to Production: Modeling, Verification, Optimization, and Protection. Heidelberg : Springer Verlag, 2016

[140] Muthuswamy, B. ; Banerjee, S.: A Route to Chaos Using FPGAs, Volume I: Experimental Observations. Basel : Springer International Publishing, 2015

[141] NeE, R. ; Prasad, R.: OFDM for Wireless Mutimedia Communications. London : Artech House Verlag, 2000

[142] Nehmzow, U.: Mobile Robotik: eine praktische Einführung. Berlin : Springer Verlag, 2002

[143] Neubig, B. ; Briese, W.: Das große Quarz-Kochbuch. Feldkirchen : FranzisVerlag, 1997

[144] Nguyen, P. ; Dittrich, J.: Praxis der feldorientierten Drehstromantriebsregelungen. Renningen-Malmsheim : Expert Verlag, 1999. - 2. Auflage

[145] Nord, Getriebebau: Dezentrale Antriebssteuerungen. https: //www.nord.com/de/produkte/elektronische-antriebstechnik/ dezentrale-antriebstechnik/dezentrale-antriebstechnik.jsp. Eingesehen zuletzt am 25.01.2020

[146] Oluaca, M. ; Hendrick, T.: Combining the ADS1202 with an FPGA Digital Filter for Current Measurement in Motor Control Applications. In: Application Report SBAA094, Texas Instruments (June 2003)

[147] ON SemiconduCTor: Datenblatt: NCP-1653. https://www.onsemi.com/ pub/Collateral/NCP1653-D.PDF. Version: 2015. - Eingesehen am 13.01.2018

[148] OpenCore: OpenCore Website. http://www.opencore.org. Version: 2016. - Eingesehen am 07.03.2018

[149] Ortseifen, A.: Entwurf von modellbasierten Anti-Windup-Methoden für Systeme mit Stellbegrenzungen, Technische Universität Darmstadt, Diss., 2012

[150] PAN, H.: Ermittlung der Rotorposition und des Feldwinkels durch indirekte 
Messung der Streuinduktivität zum Zweck der drehgeberlosen feldorientierten Regelung von Asynchronmaschinen. Düsseldorf : VDI-Verlag, 2001

[151] PanneK, J.: Modellprädiktive Regelung - Seminarskript. Universität Bayreuth, 2006 http://www.dil.biba.uni-bremen.de/fileadmin/Upload/ Skripte/2006_mpcseminar.pdf. - Eingesehen am 17.05.2017

[152] Patzelt, R. ; Schweinzer, H.: Elektrische Meßtechnik. Wien : Springer Verlag, 1996. - 2. Auflage

[153] Paul Vahle GmbH \& Co.: vPOWERB. https://vahle.de/produkte/ vpower.html. Version: Juni 2018. - Eingesehen am 19.01.2019

[154] Paulus, S.: Ein neues Konzept für die geberlose Regelung von Permanentmagnet-Synchronmaschinen für Hybrid- und Elektrofahrzeuge, Karlsruher Institut für Technologie (KIT), Diss., 2019

[155] Pavlov, A. ; Shames, I. ; Manzie, C.: Minimax strategy in approximate model predictive control. In: Automatica 111 (January 2020), S. 939-946

[156] PeHL, E.: Digitale und analoge Nachrichtenübertragung. Heidelberg : Hüthig Verlag, 2001. - 2. Auflage

[157] Perassi, H.: Feldorientierte Regelung der permanenterregten Synchronmaschine ohne Lagegeber für den gesamten Drehzahlbereich bis zum Stillstand, Universität Ilmenau, Diss., 2007

[158] Perlgrims, P.: Overview - Amba Avalon Core-Connect Wishbone. SintKatelijne-Waver : DE NAYER Instituut, 2003 http://www.pldworld.com/ _altera/html/_excalibur/add/ov-embeddedbusses.pdf. - Eingesehen am 07.03.2020

[159] Peters, V.: Selbstüberwachung und Autokalibrierung von Hall-Sensoren für die robuste Magnetfeldmessung. Saarbrücken : Shaker Verlag, 2014

[160] Podszeck, H.: Trägerfrequenz-Nachrichtenübertragung über Hochspannungsleitung. Heidelberg : Springer Verlag, 1971

[161] Pässler, R.: Rundsteuertechnik: Grundlagen, Planung, Projektierung. Erlangen : Publicis Corporate Publishing, 1994

[162] Qin, J. ; BAdgwell, T.A.: An overview of industrial Model Predictive Control Technology. In: $5^{\text {th }}$ International Conference on Chemical Process Control CPC, Tahoe City, California (1996), Nr. 93, S. 232-256

[163] QIn, J. ; BAdgwell, T.A.: A Survey of industrial Model Predictive Control Technology. In: Control Engineering Practice 11 (2003), Nr. 7, S. 733-764

[164] QuarzTechnik Daun GmBH: Quarztechnik. http://www.quarztechnik. com/. - Eingesehen am 07.01.2018

[165] Ramsden, E.: Hall-Effect Sensors: Theory and Application. Burlington : Newnes, 2006. - 2. Auflage 
[166] Rathge, C. ; Kürschner, D. ; Jumar, U.: Design methodology for high efficient inductive power transfer systems with high coil positioning flexibility. In: IEEE Transactions On Industrial Electronics 60 (2013), Nr. 01, S. 372-381

[167] RAU, M.: Nichtlineare modellbasierte prädiktive Regelung auf Basis lernfähiger Zustandsraummodelle, Technische Universität München, Diss., 2003

[168] Rawlings, J.B.: Tutorial overview of model predictive control. In: IEEE Control Systems Magazine 20 (June 2000), Nr. 03, S. 38-52

[169] Rehwald, S.: FPGAs und Mikrocontroller - Ein Vergleich. Proseminar Microcontroller und eingebettete Systeme. Technische Universität München, 2014 http://www.i6.in.tum.de/pub/ Main/TeachingWs2014ProseminarMicrocontrollerEmbedded/FPGA_ Mikrocontroller_Vergleich.pdf. - Eingesehen am 18.07.2019

[170] Reichardt, J. ; Schwarz, B.: VHDL-Synthese, Entwurf digitaler Schaltungen und Systeme. Oldenbourg Wissenschaftsverlag, 2007. - 4. Auflage

[171] Reissenweber, B.: Feldbussysteme zur industriellen Kommunikation. München : Oldenbourg Industrieverlag, 2009. - 3., vollständige überarbeitete Auflage

[172] Reijula, J. ; Gröhn, M. ; Müller, K. ; Reijula, K.: Human well-being and flowing work in an intelligent work environment. In: Intelligent Buildings International 03 (2011), Nr. 04, S. 223-237

[173] Reinhold, C.: Bestimmung elektromagnetischer Materialparameter von Werkstoffen für die kontaktlose Energie- und Datenübertragung, Otto-von-GuerickeUniversität Magdeburg, Diss., 2019

[174] Reschke, M. ; Bartels, W.: Überprüfung von Power line carrier-Systemen hinsichtlich der bidirektionalen Datenübertragung über das öffentliche Mittelund Niederspannungsnetz, Hochschule Bochum, Fachbereich Elektrotechnik und Informatik, Diplomarbeit, 1998

[175] Reuse, Design : Design and Reuse Website: IP-Core Lösungen. http://www. us.design-reuse.com. Version: 2017. - Eingesehen am 19.05.2018

[176] Rojas, P.: Theorie der neuronalen Netze: eine systematische Einführung. Berlin : Springer Verlag, 1996. - 4., korrigierter Nachdruck

[177] Rossiter, J.A.: Model-Based Predictive Control: A Ppractical Approach. London : CRC Press, 2005

[178] Ruano, A.E.: Intelligent Control Systems using Computational Intelligence. London : The Institution of Engineering and Technology, 2005 (Control Engineering Series 70)

[179] Rubiola, E.: The Leeson Effect - Phase Noise and Frequency Stability in Oscillators. http://rubiola.org/pdf-slides/2011T-IFCS-Leeson-effect.pdf.

- Eingesehen am 07.01.2018 
[180] Rudolph, G. ; Schwefel, H. P.: Evolutionäre Algorithmen - ein robustes Optimierkonzept. In: Physik Journal 50 (1994), Nr. 03, S. 236-238

[181] SahHary, B.: Elektrische Antriebe mit dauermagneterregten Maschinen im dynamischen sensorlosen Betrieb, Helmut-Schmidt-Universität (Universität der Bundeswehr Hamburg), Diss., 2008

[182] SchedLeR, D.: Kontaktlose Energieübertragung - neue Technologie für mobile Systeme. Landsberg : Verlag Moderne Industrie, 2009. - Die Bibliothek der Technik, Band 321

[183] SCHERFF, J.: Grundkurs Computernetzwerke - Eine kompakte Einführung in Netzwerk- und Internet-Technologien. Wiesbaden : Vieweg + Teubner Verlag, 2010. - 2., überarbeitete und erweiterte Auflage

[184] SchitTkowski, K. ; DAI, Y.H.: A Sequential Quadratic Programming Algorithm with Non-Monotone Line Search. In: Pacific Journal of Optimization 04 (2008), Nr. 02, S. 335-358

[185] Schnell, G. ; Wiedemann, B.: Bussysteme in der Automatisierungs- und Prozesstechnik - Grundlagen, Systeme und Anwendungen der industriellen Kommunikation. Berlin : Springer Vieweg Verlag, 2019. - 9. Auflage

[186] Schönfeld, R. ; Hofmann, W.: Elektrische Antriebe und Bewegungssteuerungen. Berlin : VDE Verlag, 2005

[187] Scholz, A. ; Becker, U. ; FAY, A.: Modellbasierte Inbetriebnahme und online-Konfiguration von Gebäudeautomationssystemen. In: atAutomatisierungstechnik 65 (2017), Nr. 09, S. 660-667

[188] Schraft, R.D. ; HäGele, M. ; Wegener, K. ; Hans, M.: Service-RoboterVisionen. München : Hanser Verlag, 2004

[189] SchröDER, D.: Leistungselektronische Schaltungen. Berlin : Springer Vieweg Verlag, 2012. - 3., überarbeitete und erweiterte Auflage

[190] SchröDER, D.: Elektrische Antriebe - Regelung von Antriebssystemen. Berlin : Springer Verlag, 2015. - 4. Auflage

[191] SchröDer, D.: Elektrische Antriebe - Grundlagen. Berlin : Springer Verlag, 2017. - 6. Auflage

[192] Schrecker, A.: Planung und Steuerung Fahrerloser Transportsysteme. Wiesbaden : Gabler Verlag, 2000

[193] Schulze, L.: FTS-Praxis: fahrerlose Transportsysteme; Planung - Realisierung - Betrieb. Gräfelfing/München : Resch Verlag, 1985

[194] Scokaert, P. ; Rawlings, J.: Feasibility issues in linear model predictive control. In: AIChE Journal 45 (1999), Nr. 8, S. 1649-1659

[195] SEW-Eurodrive GmbH \& Co KG: Handbuch: Steuerung MOVI$P L C(B)$ https://download. sew-eurodrive.com/download/pdf/11427418. pdf. Version: 2006. - Eingesehen am 17.02.2018 
[196] SEW-Eurodrive GmbH \& Co KG: Praxis der Antriebstechnik - Servotechnik. https://download. sew-eurodrive.com/download/pdf/11322802.pdf. Version: September 2006. - Eingesehen am 07.01.2018

[197] SEW-Eurodrive GmbH \& Co KG: Systembeschreibung: Kontaktlose Energieübertragung MOVITRANSB. https://download.sew-eurodrive. com/download/pdf/11493801.pdf. Version: Juni 2007. - Eingesehen am 15.06.2019

[198] SEW-Eurodrive GmbH \& Co KG: Betriebsanleitung: StationäreEnergieeinspeisung MOVITRANSB, Einspeise-Steller TPS10A. https:// download. sew-eurodrive.com/download/pdf/16754409.pdf. Version: 2009. - Eingesehen am 17.06.2017

[199] SEW-Eurodrive GmbH \& Co KG: Betriebsanleitung: Mobile Energieversorgung MOVITRANS(B) Übertragerköpfe THM10C/THM10E. https:// download. sew-eurodrive.com/download/pdf/16994000.pdf. Version: 2010. - Eingesehen am 23.06.2018

[200] SEW-Eurodrive GmbH \& Co KG: Betriebsanleitung: Stationäre Energie-Einspeisung MOVITRANS(B). https://download.sew-eurodrive. com/download/pdf/16732006.pdf. Version: August 2010. - Eingesehen am 15.06 .2019

[201] SEW-Eurodrive GmbH \& Co KG: Systemhandbuch: BedienTerminals DOP11B. https://download.sew-eurodrive.com/download/ pdf/16929608.pdf. Version: 2011. - Eingesehen am 17.06.2017

[202] SEW-Eurodrive GmbH \& Co KG: Handbuch: Konfigurations-Software für MOVIPROB. https://download.sew-eurodrive.com/download/pdf/ 19298404 .pdf. Version: 2012. - Eingesehen am 14.07.2018

[203] SEW-Eurodrive GmbH \& Co KG: Antriebs- und Applikationssteuerung MOVIPROB. https://download.sew-eurodrive.com/download/pdf/ 22488251 .pdf. Version: 2016. - Eingesehen am 26.01.2019

[204] SEW-Eurodrive GmbH \& Co KG: Systemhandbuch: MOVIDRIVER MDX60B/61B. https://download. sew-eurodrive.com/download/pdf/ 23538449.pdf. Version: 2017. - Eingesehen am 05.01.2019

[205] SEW-Eurodrive GmbH \& Co KG: Mobile Assistenzsysteme. https: //www.sew-eurodrive.de/automatisierung/fabrikautomatisierung/ mobile-assistenzsysteme/mobile-assistenzsysteme.html.

Version: Januar 2019. - Eingesehen am 14.09.2019

[206] Sinsel, A.: Das Internet der Dinge in der Produktion: Smart Manufacturing für Anwender und Lösungsanbieter. Berlin : Springer Vieweg Verlag, 2020

[207] Soeterboek, R.: Predictive Control- A Unified Approach, Technische Universität Delft, Diss., 1990 
[208] Specovius, J.: Grundkurs Leistungselektronik. Wiesbaden : Verlag Vieweg + Teubner, 2009. - 3., aktualisierte und erweiterte Auflage

[209] Steiner, W. ; PolednA, S.: Fog computing as enabler for the Industrial Internet of Things. In: Elektrotechnik und Informationstechnik 133 (2016), Nr. 07, S. 310-314

[210] Steven, M. ; Dörseln, J.N.: Smart Factory: Einsatzfaktoren - Technologie Produkte. Stuttgart: W. Kohlhammer Verlag, 2020

[211] STMicroelectronics N.V.: Datenblatt: STM32F031x6-Serie. https://dtsheet.com/doc/1400009/datasheet---stmicroelectronics. Version: 2015. - Eingesehen am 07.03.2020

[212] Texas Instruments: Datenblatt: DP83640 Precision PHYTER - IEEE 1588 Precision Time Protocol Transceiver. https://www.ti.com/product/ DP83640. Version: Januar 2015. - Eingesehen am 08.07.2018

[213] Texas Instruments: Datenblatt: SN65HVD233 CAN Transceiver. https: //www.ti.com/lit/ds/symlink/sn65hvd233. Version: 2017. - Eingesehen am 22.07 .2018

[214] Texas Instruments: Datenblatt: SN75HVD11D RS485 Transceiver. https: //www.ti.com/product/SN75HVD11. Version:2017. - Eingesehen am 13.07.2019

[215] Texas Instruments: Datenblatt: C2000-MCU-Serie. http://www.ti.com/ de-de/microcontrollers/overview.html. Version:2020. - Eingesehen am 07.03.2020

[216] Thrun, S. ; Burgard, W. ; Fox, D.: Probabilistic robotics. Cambridge : MIT Press, 2006

[217] Tietze, U. ; Schenk, C.: Halbleiter-Schaltungstechnik. Heidelberg : Springer Verlag, 2002. - 12. Auflage

[218] TÖPFER, H.: Wissensspeicher Fluidtechnik: hydraulische und pneumatische Antriebs- und Steuerungstechnik. Leipzig : Fachbuchverlag, 1988

[219] TREnkle, A.: KARIS PRO - Autonomer Materialtransport für flexible Intralogistik. Abschlussbericht des BMBF-Verbundforschungsprojektes. http: //www.karispro.de/. - Eingesehen am 14.03.2020

[220] Trenkle, A.: Entwurfsmuster für Fahrerlose Transportsysteme, Karlsruher Institut für Technologie (KIT), Diss., 2018

[221] URkowitz, H.: Signal theory and random processes. London : Artech House Verlag, 1983

[222] VDI-RICHTLINIEN: VDI 2206 - Entwicklungsmethodik für mechatronische Systeme. https://www.vdi.de/richtlinien/details/ VDI-2206-Entwicklungsmethodik-fuer-Mechatronische-Systeme.

Version: 2004. - Eingesehen am 16.05.2020 
[223] Virtual Component: Virtual Component Exchange Website. http://www. thevcx.com. Version: 2016. - Eingesehen am 29.07.2018

[224] Vogel-Heuser, B. ; Bauernhansl, T. ; Hompel, Michael ten: Handbuch Industrie 4.0. Berlin : Springer Verlag, 2018. - 2. Auflage

[225] Vogel-Heuser, B. ; Bauernhansl, T. ; Ten Hompel, M.: Handbuch Industrie 4.0: Produktion, Automatisierung und Logistik. Berlin : Springer Verlag, 2016

[226] Wangenheim, L.: Analoge Signalverarbeitung: Systemtheorie, Elektronik, Filter, Oszillatoren, Simulationstechnik. Wiesbaden : Vieweg + Teubner Verlag, 2010

[227] Weber, W.: Industrieroboter: Methoden der Steuerung und Regelung. München : Fachbuchverlag Leipzig im Carl Hanser Verlag, 2017. - 3., neu bearbeitete Auflage

[228] WeIkeR, K.: Evolutionäre Algorithmen. Wiesbaden : Springer Vieweg Verlag, 2015. - 3., überarbeitete und erweiterte Auflage

[229] Wellers, M.: Nichtlineare Modellgestützte Prädiktive Regelung auf Basis von Wiener- und Hammerstein-Modellen, RWTH-Aachen, Diss., 1998

[230] Werner, M.: Nachrichtentechnik: eine Einführung für alle Studiengänge. Wiesbaden : Springer Vieweg Verlag, 2017. - 8., vollständig überarbeitete und erweiterte Auflage

[231] Wittmuess, W.: Ansätze zur dynamischen Modellierung thermischer Aktoren in Laserresonatoren. Aachen : Shaker Verlag, 2017

[232] Witтpahl, V.: Künstliche Intelligenz: Technologie, Anwendung, Gesellschaft. Berlin : Springer Verlag, 2019

[233] Wolf, W.: Entwicklung eines agentenbasierten Steuerungssystems zur Materialflussorganisation im wandelbaren Produktionsumfeld, Friedrich-AlexanderUniversität Erlangen-Nürnberg, Diss., 2009

[234] Xilinx Inc. Corporation: MicroBlaze Soft Processor Core. https://www. xilinx.com/products/design-tools/microblaze.html. - Eingesehen am 13.01 .2018

[235] YÜZAY, R.: Motorsteuerung auf einem programmierbaren Chip. In: Elektronik 57 (2008), Nr. 26, S. 41-47

[236] Zheng, T.: Model Predictive Control. London : IntechOpen Limited, 2011 https://www.intechopen.com/books/ advanced-model-predictive-control. - Eingesehen am 07.01.2018

[237] Ziemann, O. ; Krauser, J. ; Zamzow, P.E. ; Daum, W.: POF-Handbuch Optische Kurzstrecken-Übertragungssysteme. Berlin : Springer Verlag, 2007. 2., bearbeitete und ergänzte Auflage

[238] Zimmermann, M.: Energieverteilnetze als Zugangsmedium für Telekommuni- 
kationsdienste. Aachen : Shaker Verlag, 2000 



\section{A. Technische Daten des Funktionsmusters}

Durchmesser Behälterdrehtisch:

$d_{B d}=3000 \mathrm{~mm}$

Anzahl der Drehteller:

Masse Behälterdrehtisch:

Massenträgheit Behälterdrehtisch:

$D t_{A n z}=4$

$m_{B d}=320 \mathrm{~kg}$

Gesamte Massenträgheit des Drehtisches mit Aufbauten:

$J_{B d}=360 \mathrm{kgm}^{2}$

Maximaldrehzahl Behälterdrehtisch:

$J_{B d_{G e s}}=404 \mathrm{kgm}^{2}$

Beschleunigungszeit Drehtischmotor auf Nenndrehzahl:

$n_{B d}=20 \mathrm{U} / \mathrm{min}$

Etikettierleistung bei Nenndrehzahl:

Durchmesser der Drehteller:

Masse je Drehteller:

Maximaldrehzahl der Drehteller:

$t_{B d}=50 \mathrm{~s}$

$P_{E t}=70.000 S t / h$

$d_{D t}=80 \mathrm{~mm}$

$m_{D t}=2.5 \mathrm{~kg}$

$n_{D t}=1500 \mathrm{U} / \mathrm{min}$

Beschleunigungszeit Drehtellermotoren auf Nenndrehzahl:

Abstand zwischen den Drehtellern:

Durchmesser der Antriebsrolle des Etikettiermoduls:

$t_{D t}=0.25 \mathrm{~s}$

$D t_{A b s t}=60 \mathrm{~mm}$

$d_{E t}=100 \mathrm{~mm}$

$d_{E t_{e x}}=50 \mathrm{~mm}$

Durchmesser der externen Rolle des Etikettiermoduls:

Maximaldrehzahl der Antriebswelle des Etikettiermoduls:

$n_{E t}=1750 \mathrm{U} / \mathrm{min}$

Beschleunigungszeit Etikettiermodul auf Nenndrehzahl:

Länge des Etikettes:

Länge der Etikettierstrecke:

$t_{E t}=0.25 \mathrm{~s}$

$s_{E t}=100 \mathrm{~mm}$

$s_{E t_{S t r}}=50 \mathrm{~mm}$

\section{Antriebskomponenten - Motoren und Getriebe:}

Drehtischmotor CFM71S mit Flachgetriebe FA47:

Drehtellermotoren CMD55L, Direktantrieb:

$M_{0}=5 \mathrm{Nm}$

Etikettiermodulmotor CMD93M, Direktantrieb:

$M_{0}=0.9 \mathrm{Nm}$

$M_{0}=4.2 \mathrm{Nm}$ 



\section{B. Lineare Oszillatoren}

\section{B.1. Wirkungsweise von Oszillatoren}

Lineare- oder harmonische Oszillatoren sind elektronische Schaltungen, die ein kontinuierliches bzw. sinusförmiges Ausgangssignal mit konstanter Amplitude erzeugen. Bei der Erzeugung einer solchen ungedämpften Schwingung wird kein zusätzliches Eingangssignal benötigt. Neben den genannten Sinusoszillatoren, die sinusförmige Signale erzeugen, unterscheidet man ferner sogenannte Impulsoszillatoren. Impulsoszillatoren werden zur Erzeugung beliebiger anderer Signalformen wie z.B. einer Rechteckform oder einer Dreieckform verwendet. Kennwerte der Oszillatoren sind [143]:

- Frequenzkonstanz

- Stabilität der Amplitude

- Spektrale Reinheit des Signals.

Was den technischen Aufbau und die Realisierung bzw. die Erzeugung einer schwingungsfähigen Schaltung betrifft, können vier Funktionsprinzipien unterschieden werden [143]. Erzeugung einer Schwingung durch:

- Digitale Bauteile

- Relaxationsoszillatoren

- Zweipol-Oszillatoren

- Vierpol-Oszillatoren.

Ein verbreitetes Prinzip bei der digitalen Erzeugung einer Sinusschwingung ist das sogenannte DDS-Verfahren (engl.: Direct Digital Synthesis). Hier wird das Sinussignal durch einen Zähler, einen Speicherbaustein mit den hinterlegten Sinuswerten und einem D/A-Wandler erzeugt. 
Bei den Relaxationsoszillatoren wird die Frequenz durch die gesteuerte Ladung und Entladung eines Kondensators in einem RC-Glied bestimmt. Dabei werden die Zeitkonstanten der Umladevorgänge am Kondensator zur Festlegung der Periodendauer genutzt.

Zweipol-Oszillatoren, auch als „RLC-Resonanzkreis mit NIC“ (engl.: Negative Impedance Converter) bezeichnet, basieren auf dem Prinzip der Entdämpfung des LCSchwingkreises. Der Schwingkreis wird dabei durch einen kurzzeitigen Spannungsoder Stromimpuls angeregt. Reale Widerstände bzw. verlustbehaftete Bauteile dämpfen die entstehende Schwingung jedoch wieder ab. Die Entdämpfung des Schwingkreises wird dann durch Einbringen eines aktiven negativen Zusatzwiderstandes erreicht, der die Gesamtverluste der Schaltung wieder ausgleicht. Der negative differentielle Widerstand wird schaltungstechnisch in der Regel durch den Einbau einer Tunneldiode realisiert.

Neben den Zweipol-Oszillatoren, die in der Praxis geringere Bedeutung haben, erfolgt die Erzeugung einer ungedämpften sinusförmigen Schwingung meist mit einem Vierpol-Oszillator. Vierpol-Oszillatoren bestehen aus einer Verstärkerschaltung, welche die Aufgabe der Entdämpfung übernimmt und einem frequenzabhängigen Rückkopplungsnetzwerk. Die Festlegung der Schwingfrequenz erfolgt im passiven Filternetzwerk des Rückkopplungszweiges. Die Wirkungsweise einer rückgekoppelten Verstärkerschaltung ist in Abbildung B.1 dargestellt.

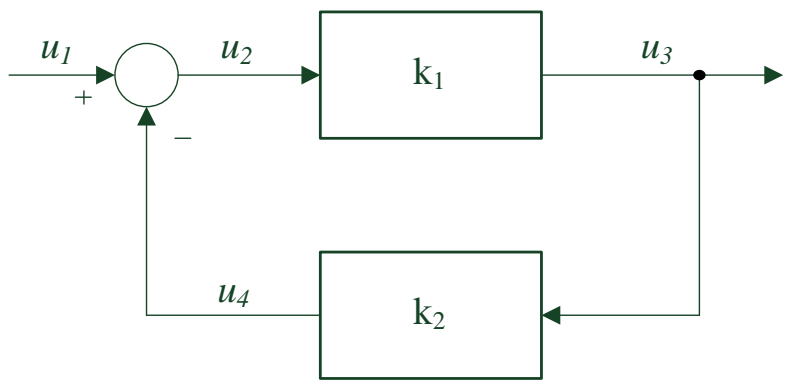

Abbildung B.1.: Rückgekoppelte Verstärkerschaltung [226].

Dabei ist $u_{1}$ das Eingangssignal der Gesamtschaltung. $u_{2}$ und $u_{3}$ sind die Ein- und Ausgangssignale des Verstärkers. $u_{4}$ ist das Ausgangssignal bzw. das rückgekoppelte Signal des Filternetzwerkes. Weiterhin sind der komplexe Verstärkungsfaktor $\mathbf{k}_{1}$ und der komplexe Rückkopplungsfaktor $\mathbf{k}_{2}$ in der Zeichnung dargestellt. Die Übertragungsfunktion für das System ergibt sich entsprechend Gl. (B.1).

$$
\frac{u_{3}}{u_{1}}=\frac{\mathbf{k}_{1}}{1+\mathbf{k}_{S}}=\mathbf{k}_{V}
$$


$\mathbf{k}_{V}$ ist ein Maß für die Gesamtverstärkung der Schaltung. $\mathbf{k}_{S}=\mathbf{k}_{1} \mathbf{k}_{2}$ steht für die Schleifenverstärkung. Für $\mathbf{k}_{S}>0$ entsteht Gegenkopplung: $\mathbf{k}_{V}<\mathbf{k}_{1}$. Für $\mathbf{k}_{S}<0$ entsteht Mitkopplung: $\mathbf{k}_{V}>\mathbf{k}_{1}$. Für $\mathbf{k}_{S} \rightarrow-1$ geht $\mathbf{k}_{V} \rightarrow \infty$, d.h. bei endlichem Ausgangssignal von $u_{3}$ geht das Eingangssignal $u_{1} \rightarrow 0$. Solche Schaltungen werden als Oszillatoren bezeichnet.

Um eine entstandene Schwingung in Abbildung B.1 aufrecht zu erhalten, muss die Schaltung bestimmte Bedingungen genügen. Sind Verstärker- und Rückkopplungszweig im regelungstechnischen Sinne stabil, kann das Nyquist-Kriterium angewendet werden. Das Nyquist-Kriterium besagt, dass eine Oszillation nur möglich ist, wenn der Betrag der offenen Schleife gleich 1 ist und die Phasenverschiebung $180^{\circ}$ beträgt. Es gelten dann für die Schwingfähigkeit einer invertierenden Verstärkerstufe Gl. (B.2) und Gl. (B.3) [25], [226].

$$
\begin{aligned}
\left|\mathbf{k}_{1} \mathbf{k}_{2}\right| & =1 . \\
\arg \left(\mathbf{k}_{1} \mathbf{k}_{2}\right) & =180^{\circ} .
\end{aligned}
$$

Da die Bauteile der Schaltung Toleranzen und temperaturbedingte Schwankungen aufweisen, muss der Betrag der tatsächlichen Schleifenverstärkung von $\mathbf{k}_{S}>1$ sein. Aus dem genannten Grund werden in der Regel im Verstärkerzweig nichtlineare Bauteile eingesetzt, die die Amplitude wieder begrenzen bzw. stabilisieren. Der Rückkopplungszweig weist dabei eine frequenzselektive Funktion auf und muss der Phasenbedingung genügen. Die Phasenbedingung muss bei einem nichtinvertierenden Verstärker bei der Schwingungsfrequenz $f_{0}$ ein ganzzahliges Vielfaches von $360^{\circ}$ betragen. Frequenzselektive Elemente werden häufig durch Phasenschieber oder LCSchwingkreise realisiert.

Phasenschieber sind meist als passive RC-Netzwerke 3. oder 4. Ordnung aufgebaut, je nach Grad der Phasenverschiebung der einzelnen RC-Stufen. LC-Oszillatoren werden vor allem zur Erzeugung von Sinusschwingungen höherer Frequenz verwendet. Sie finden häufig in Oszillatoren Anwendung und weisen die Charakteristik eines Bandpasses auf. Allerdings weisen LC-Oszillatoren eine schlechte Frequenzkonstanz auf. Anstelle der LC-Schwingkreise werden deswegen oft Schwingquarze verwendet. Schwingquarze im Rückkopplungszweig eines Oszillators können eine sehr hohe Schwingungsgüte erreichen, sie werden deshalb im Bereich der Signalverarbeitung vielfältig eingesetzt. Im nächsten Abschnitt wird kurz auf den Aufbau und die Eigenschaften von Quarzoszillatoren eingegangen. 


\section{B.2. Eigenschaften von Schwingquarzen}

Werden bestimmte kristalline Materialien mechanisch verformt oder deformiert, entsteht durch Ladungsverschiebung im Kristall eine entsprechende proportionale elektrische Spannung. Die Spannung ist an der Oberfläche des Kristalls abgreifbar. Entdeckt wurde der Effekt bereits im Jahre 1880 durch die Gebrüder Curie. Dieser sogenannte direkte piezoelektrischer Effekt ${ }^{1}$ [74] oder auch kürzer Piezoeffekt ist gleichfalls umkehrbar. Beim umgekehrten oder inversen Piezoeffekt [74] wird durch Anlegen einer elektrischen Spannung eine elastische Verformung des kristallinen Materials bewirkt, es weist die Eigenschaften eines mechanischen Schwingers auf.

Eines der wichtigsten und bekanntesten Materialien mit Piezoeigenschaften ist Quarz ${ }^{2}$. Quarze, hier speziell Schwingquarze, wurden anfänglich aus der einkristallinen Variante des Siliziumdioxid $\left(\mathrm{SiO}_{2}\right)$ hergestellt. Eine solche Form, auch Bergkristall genannt, kommt allerdings in der Natur nur sehr selten vor. Der Kristall muss dabei vor allem frei sein von Verwachsungen und Verzwilligungen, Zonen mit verpoltem piezoelektrischen Effekt. Heute werden Schwingquarze überwiegend synthetisch hergestellt durch Züchtung in vertikalen Autoklaven.

Wie bei allen mechanischen Schwingern ist die Resonanzfrequenz des Schwingquarzes von den Materialkonstanten, vor allem aber von den mechanischen Abmessungen abhängig. Dabei kommen Schwingungsformen wie Biegung, Dehnung, Flächenscherung und Dickenscherung zur Anwendung. In der Elektronik werden immer mehr Oszillatoren mit hoher Genauigkeit und Frequenzkonstanz benötigt, dabei stabilisiert der Schwingquarz als zentrales Element den Schaltungsaufbau. Als elektromechanischer Resonator weist das kristalline Material dabei eine gute mechanische Stabilität sowie geringe Dämpfung und Temperaturabhängigkeiten auf.

\section{Quarzschnitte}

Gerade im Bereich der Resonanzfrequenz sollte die Temperaturabhängigkeit des Schwingquarzes möglichst klein sein. Neben dem minimalen Temperaturgang wünscht man sich gleichzeitig ein gutes Verhalten für die elektrische Anregung. Solche Eigenschaften kann man durch einen gezielten Quarzschnitt beeinflussen. Abbildung B.2 zeigt einige typische Quarzschnitte wie die Schwingkörper optimal aus dem Quarzkristall herausgeschnitten werden.

Neben den in der Abbildung B.2 gezeigten Schnittwinkeln gibt es noch eine Vielzahl anderer Varianten sowie diverse Spezialschnitte.

\footnotetext{
${ }^{1}$ von Piezo - Griechisch: ich drücke

${ }^{2}$ genaue wissenschaftliche Bezeichnung ist Tiefquarz oder $\alpha$-Quarz
} 


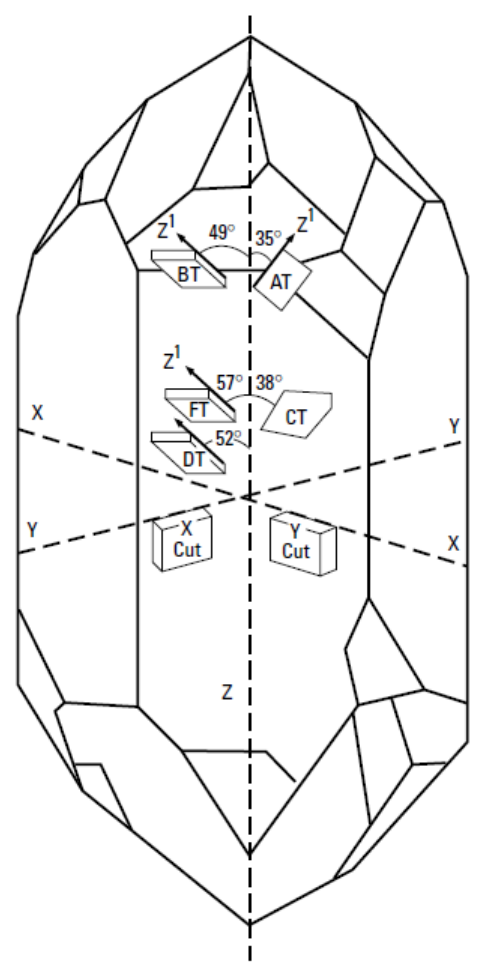

Abbildung B.2.: Schnittlage der Quarzplättchen im Kristall [77].

\section{Temperaturabhängigkeit}

Abhängig vom gewählten Schnitt kann die Temperaturabhängigkeit der Frequenz von Schwingquarzen durch eine Gleichung zweiten Grades, Gl. (B.4) oder dritten Grades, Gl. (B.5) [143] dargestellt werden.

$$
\begin{aligned}
& \frac{\Delta f_{T}}{f}=a_{T}\left(T-T_{0}\right)+b_{T}\left(T-T_{0}\right)^{2} . \\
& \frac{\Delta f_{T}}{f}=a_{T}\left(T-T_{0}\right)+b_{T}\left(T-T_{0}\right)^{2}+c_{T}\left(T-T_{0}\right)^{3} .
\end{aligned}
$$

Außer bei AT-, SC-, und GT-Schnitten weisen Schwingquarze einen parabelförmigen Verlauf der Temperaturabhängigkeit auf. Dabei werden die Konstanten $a_{T}, b_{T}$ und $c_{T}$ sehr stark vom Schnittwinkel und den Fertigungstoleranzen bestimmt. Die Bezugstemperatur wird mit $25^{\circ} \mathrm{C}$ vorgegeben. Die Angabe eines Temperaturkoeffizienten $\delta f / \delta T$ ist hier nicht zweckmäßig, da sich an den Wendestellen der Gleichungen 
der differentielle Wert $=0$ ergibt. In der Literatur wird deswegen eine maximale Frequenzabweichung über einen bestimmten Temperaturbereich betrachtet.

\section{Dickenscherungsschwinger}

Im Bereich von 1 . . $250 \mathrm{MHz}$ werden überwiegend Dickenscherungsschwinger (DSS) eingesetzt [143]. Durch seine Bewegungsart ist der DSS besonders stabil gegen äußere Einflüsse. Der DSS wird im AT-Schnitt hergestellt, weist daher einen kubischen Frequenz-Temperaturverlauf auf. Durch kleine Veränderungen des Schnittwinkels ist es möglich, den Temperaturgang so zu verschieben, dass für die Resonanzfrequenz der Umkehrpunkt bei Raumtemperatur liegt (Abbildung B.3). Für jeden Arbeitstemperaturbereich ergibt sich somit ein optimaler Schnittwinkel.

Wie in Abbildung B.3 zu sehen ist, zeichnet sich der SC-Schnitt (engl.: StressCompensated) durch einen flacheren Temperaturgang aus als der AT-Schnitt. Er ist allerdings aufwändiger in der Herstellung.
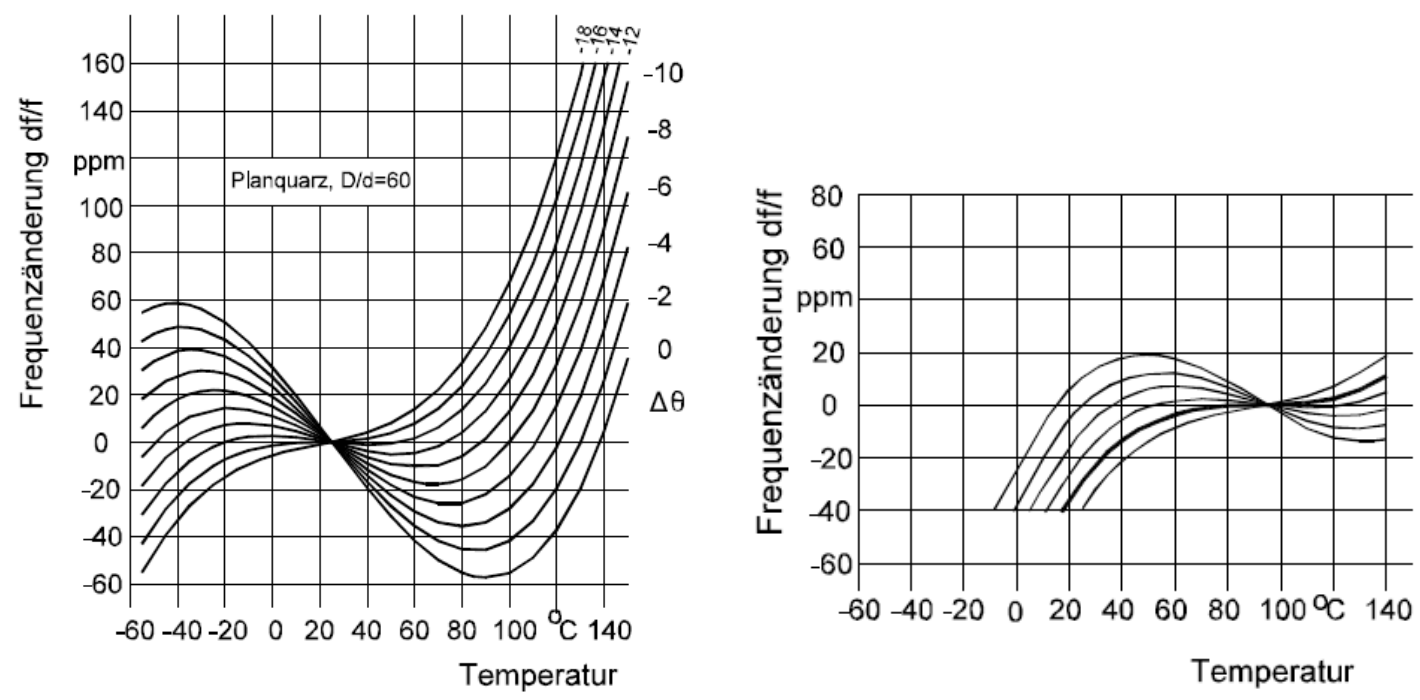

(a) AT-Schnitt.

(b) SC-Schnitt.

Abbildung B.3.: Vergleich Temperaturverlauf bei AT- und SC-Schnitt [143].

\section{Alterung}

Verändern sich die Resonanzfrequenz bzw. die Eigenschaften des Schwingquarzes über einen längeren Zeitraum, spricht man von einer Alterung des Quarzes. Eine solche Frequenzdrift kann materialbedingte oder auch konstruktionsbedingte Ursachen 
haben. So können kristalline Verunreinigungen durch die Wanderung von eingebauten Fremdatomen hervorgerufen werden. Bei synthetisch hergestellten Quarzen können ferner durch den beschleunigten Herstellungsprozess beim Züchten mechanische Spannungen entstehen. Andere negative Einflüsse entstehen durch die Halterung des Quarzes im Gehäuse. Bei hohen mechanischen Anforderungen an den Schwingquarz übt dieser einen erhöhten Druck auf den Resonator aus. Weitere Einflüsse entstehen durch Diffusion von Wasserdampf in die Gitterstruktur des Quarzes, durch Oxidationsprozesse und durch das Eindringen von Gasen. Wichtig ist es daher, den Quarz in einem hermetisch abgeschlossenen Gehäuse unterzubringen. Dafür kommen meist Metall- und Keramikgehäuse zur Anwendung. Kunststoffe sind wegen ihrer Wasserdampfdurchlässigkeit weniger geeignet.

\section{Kurzzeitstabilität}

Die Kurzzeitstabilität definiert die kurzzeitigen Frequenzschwankungen um den statistischen Mittelwert der Resonanzfrequenz des Oszillators im Bereich von Sekunden bis Minuten. Die Kurzzeitstabilität kann dabei im Zeitbereich und im Frequenzbereich betrachtet werden [143]. Gl. (B.6) beschreibt ein Sinussignal mit Rauschen im Zeitbereich.

$$
U(t)=\left(U_{0}+\epsilon(t)\right) \cos \left[2 \pi f_{N}(t)+\varphi_{0}+\Delta \varphi(t)\right] .
$$

In Gl. (B.6) können Schwankungen der Amplitude $\epsilon(t)$ und Schwankungen durch Veränderungen der Phasenlage $\Delta \varphi(t)$, d.h. durch Phasenrauschen auftreten. Unter Vernachlässigung der Amplitudenschwankung, was hier nicht Gegenstand der Untersuchung sein soll, ist die Oszillatorfrequenz dann nur noch abhängig vom Phasenrauschen. Unter Zuhilfenahme von statistischen Methoden kann die Quarzfrequenz dabei als eine vom Phasenrauschen abhängige Zufallsvariable betrachtet werden. Die Varianz berechnet sich gemäß Gl. (B.7) [143].

$$
\begin{aligned}
\sigma^{2}\left(N_{M}, \tau_{0}\right) & =\frac{1}{N_{M}-1} \sum_{k=1}^{N_{M}}\left(y_{d}(k)-\bar{y}_{d}\right)^{2} . \\
y_{d}(k) & =\frac{f(k)-f_{0}}{f_{0}} .
\end{aligned}
$$

Der Wert $y_{d}(k)$ berechnet sich aus der Differenz gemäß Gl. (B.8) bezogen auf den Mittelwert der Nominalfrequenz $f_{0}$. Die Messung erfolgt zwischen dem festen Zeitintervall $\tau_{0} . N_{M}$ definiert die Anzahl der gemessenen Werte für $y_{d}(k), \bar{y}_{d}$ den Mittelwert 
der Daten aus den Messungen für $y_{d}(k)$. Nachteilig bei der Bestimmung der Standardabweichung bzw. Varianz ist, dass ein langsames Wegdriften der Oszillatorfrequenz von seiner Nennfrequenz das Messergebnis verfälscht. Das Phasenrauschen eines Oszillators im Zeitbereich wird deshalb in der Regel durch die sogenannte Allan-Varianz dargestellt.

\section{Allan-Varianz}

Die Allan-Varianz, benannt nach David Allan, kennzeichnet in der Literatur ein Maß für die Stabilität von Oszillatoren und Uhren bzw. bewertet die Frequenzkonstanz innerhalb eines betrachteten Zeitraumes. Sie wird in der Fachliteratur auch als Zweiwert- oder auch als Paar-Varianz bezeichnet. Die Allan-Varianz ist gemäß Gl. (B.9) definiert [143], [3].

$$
\begin{aligned}
\sigma^{2}\left(N_{M}, 2, \tau_{0}\right) & =\frac{1}{2\left(N_{M}-1\right)} \sum_{k=1}^{N_{M}}\left(y_{d a}(k+1)-y_{d a}(k)\right)^{2} . \\
y_{d a}(k) & =\frac{f(k+1)-f(k)}{f_{0}} .
\end{aligned}
$$

Im Unterschied zur Gl. (B.7) wird nicht der Mittelwert aller Stichproben zur Differenzbildung herangezogen, sondern es wird die Differenz zweier nachfolgender Stichproben betrachtet - dadurch wird ein Wegdriften der Oszillatorfrequenz von der Resonanzfrequenz berücksichtigt. Werden mehrere Messungen mit unterschiedlichen Samplingraten für $\tau_{0}$ durchgeführt, wird ein typischer Kurvenverlauf wie in Abbildung B.4 gezeigt deutlich.

Aus der Steigung des linearen Teiles der Kurve kann auf die Art der Varianz geschlossen werden. Eine kleinere Varianz bedeutet dabei eine höhere Frequenzstabilität. Für kleine $\tau_{0}$ verschlechtert sich die Frequenzkonstanz bedingt durch den höheren anteiligen Rauschanteil. Im mittleren Bereich, bei einer oder mehreren Sekunden, sind die Frequenzschwankungen am geringsten. Für größere Intervallzeiten von $\tau_{0}$, im rechten Teil der Kurve erkennbar, verschlechtert sich die Frequenzkonstanz durch das Wegdriften der Frequenz wieder.

Neben der Darstellung im Zeitbereich kann das Phasenrauschen eines Oszillators auch im Frequenzbereich durch sein Leistungsdichtespektrum abgebildet werden. Abbildung B.5 zeigt beispielhaft ein typisches Frequenzspektrum. 


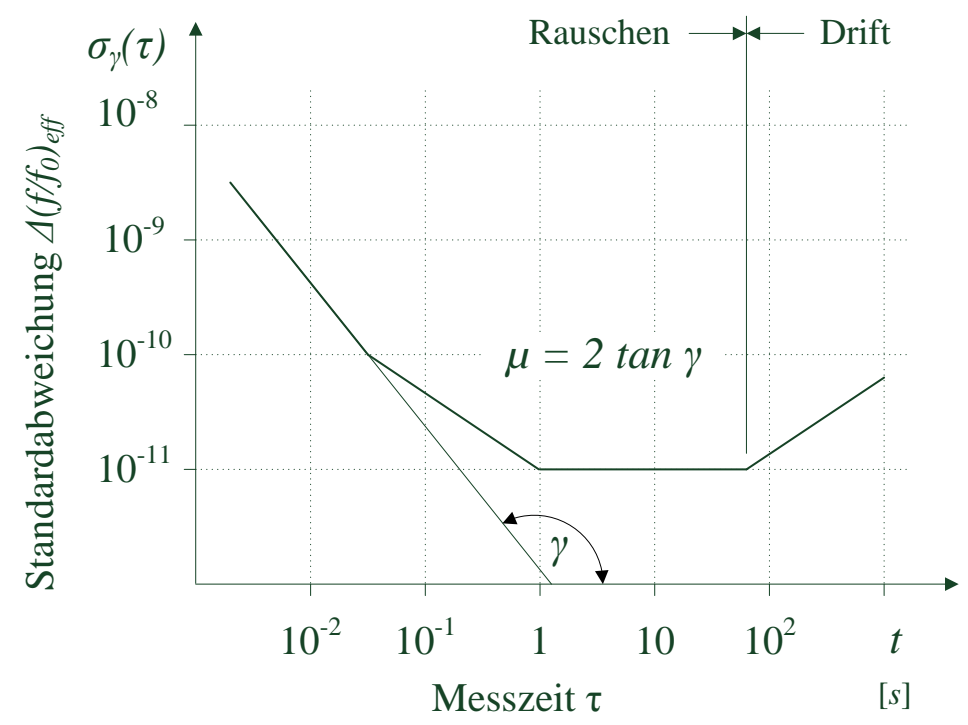

Abbildung B.4.: Darstellung der Allan-Varianz [143].

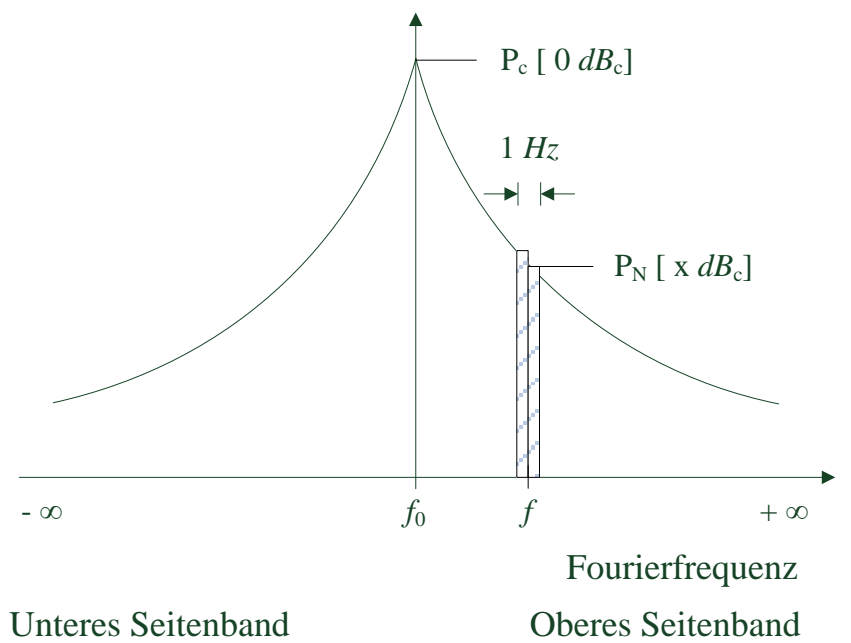

Abbildung B.5.: Frequenzspektrum eines Oszillators [143].

Durch das Phasenrauschen bilden sich im Frequenzspektrum beidseitig der Nominalfrequenz zusätzliche Frequenzbänder aus. Die Leistung der Stör- bzw. Rauschfrequenzen fällt dabei rechts und links von $f_{0}$ stark ab. Qualitativ hochwertige Oszillatoren zeichnen sich durch schmalbandige Frequenzbänder um $f_{0}$ aus. Weitere Einflussfaktoren sind Verstärkerrauschen und das thermische Rauschen im Rückkopplungszweig der Oszillatorschaltung. 


\section{Ersatzschaltbild und Resonanzfrequenzen}

Die Eigenschaften des Schwingquarzes bzw. die eines mechanischen Schwingers lassen sich zum besseren Verständnis in einem äquivalenten elektrischen Schaltbild gemäß Abbildung B.6 darstellen.

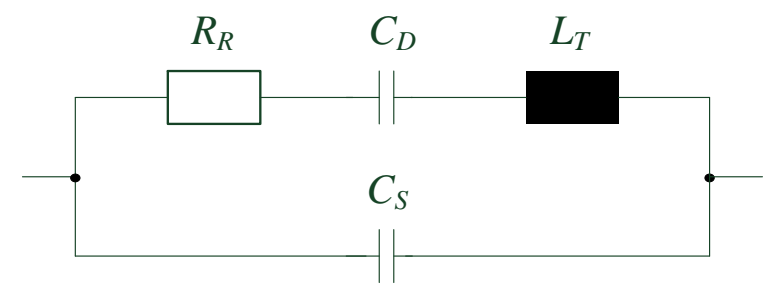

Abbildung B.6.: Ersatzschaltbild eines Schwingquarzes [143].

Der Resonanzwiderstand $R_{R}$ bildet dabei die mechanischen Schwingungsverluste im Quarz ab. Die dynamische Kapazität $C_{D}$ steht für die elastischen Eigenschaften. Durch die Induktivität $L_{T}$ wird die Trägheit der Masse des Quarzmaterials abgebildet. Die statische Kapazität $C_{S}$ ist dem Serienresonanzkreis parallel geschaltet, sie repräsentiert die messbare Kapazität an den Halterungen des Quarzes und auftretende Streuverluste. Bei hohen Frequenzen muss das vereinfachte Schaltbild gemäß Abbildung B.6 allerdings erweitert werden, um den verstärkten Einfluss durch die Halterungselemente und die Übergangswiderstände der Elektrodenanschlüsse abzubilden. Neben der unteren Resonanzfrequenz des Serienschwingkreises $f_{\text {Ser }}$, Gl. (B.11) entsteht durch die Parallelschaltung von $C_{S}$ eine weitere etwas höhere Parallelresonanzfrequenz.

$$
f_{S e r}=\frac{1}{2 \pi \sqrt{L_{T} C_{D}}}
$$

Meistens wird der Schwingquarz schaltungstechnisch in eine sogenannte Phi-Schaltung integriert [143]. Dabei wird der Schwingquarz in Reihe mit einer Lastkapazität $C_{L}$ geschaltet. Durch die Serienschaltung des Quarzes mit der Lastkapazität verschiebt sich die Resonanzfrequenz zu einer höheren Frequenz und wird dann als Lastresonanzfrequenz $f_{L}$, Gl. (B.12), bezeichnet. Auch die parallele Beschaltung der kapazitiven Last zum Reihenschwingkreis ist üblich [143].

$$
f_{L}=f_{S e r}+f_{S e r} \frac{C_{D}}{2\left(C_{S}+C_{L}\right)} .
$$

Durch die schaltungstechnische Integration der zusätzlichen Lastkapazität $C_{L}$ ist es möglich, die Resonanzfrequenz eines Oszillators zu verändern. Hierfür eignen sich 
Trimkondensatoren oder Kapazitätsdioden. In den technischen Datenblättern von Schwingquarzen wird neben der Lastresonanzfrequenz $f_{L}$ auch die dafür notwendige Lastkapazität $C_{L}$ angegeben. Die maximal erreichbare Schwingungsgüte ist frequenzabhängig und nimmt mit steigender Frequenz ab. Die Schwingungsgüte $Q_{S}$ ist gemäß Gl. (B.13) definiert [143].

$$
Q_{S}=\frac{1}{\omega C_{D} R_{R}}=\frac{\omega L_{T}}{R_{R}}
$$

Unter Vernachlässigung der Umgebungsbedingungen, einem ausreichend großen Resonatordurchmesser des Quarzes und der Unterbringung in einem evakuierten Gehäuse ist die Güte ferner durch die viskosen Verluste des Materials begrenzt. Bei gegebenen Materialkonstanten werden Schwingquarze mit hoher Schwingungsgüte, bedingt durch die Frequenzabhängigkeit, in der Regel im Frequenzbereich von $2 \ldots 10 \mathrm{M} \mathrm{Hz}$ [143] hergestellt. Zur Erzeugung höherer Frequenzen werden meist elektronische Schaltungen (PLL, engl.: Phase Locked Loop) zur Frequenzvervielfachung eingesetzt.

\section{B.3. Beschreibung unterschiedlicher Oszillatorklassen}

Ausschlaggebend für die Güte eines Oszillators ist die Qualität des verwendeten Schwingquarzes selbst. Neben dem Schnittwinkel bestimmt die Art des Quarzschnittes und die Realisierung der Verstärkerschaltung im Rückkopplungszweig des Oszillators die Güte. Die Verschlechterung der Schwingungsgüte durch die Schwingungsverstärkung im Rückkopplungszweig kann rechnerisch durch das Leeson-Modell [179] abgeschätzt werden. Die am Markt erhältlichen Oszillatoren werden dabei in unterschiedliche Klassen eingeteilt [143].

Für unterschiedliche Anwendungsfälle stehen Oszillatoren mit unterschiedlichen Merkmalen und unterschiedlicher Güte zur Verfügung. Die in der Tabelle B.1 angegebenen Werte für die Frequenz und für die Genauigkeit stellen typische Werte dar [164]. Unter der Genauigkeit ist hier die Frequenzänderung $\Delta f / f$ zu verstehen. Der Wert der Genauigkeit ist zusätzlich abhängig vom Betriebstemperaturbereich, in dem der Oszillator betrieben wird.

PXO-Quarzoszillatoren (engl.: Package Crystal Oscillator), meist als XO-Oszillatoren bezeichnet, sind einfache Oszillatoren, die vor allem in vielen digitalen Schaltkreisen, in der Regel als SMD-Bauteil (engl.: Surface Mounted Devices), zur Takterzeugung eingesetzt werden. Der Aufbau ist gekennzeichnet durch den Quarz selbst und der dazugehörigen elektronischen integrierten Schaltung. Der Oszillator arbeitet auf seiner quarzabgestimmten Frequenz. Eine Korrektur der Resonanzfrequenz, bedingt durch den Temperaturgang des Quarzes, findet nicht statt. 
Tabelle B.1.: Klassen von Oszillatoren [143], [164].

\begin{tabular}{lllll}
\hline Klasse & Typ & Einsatzgebiet & Frequenz & Genauigkeit \\
\hline PXO & Standard Quarzoszillator & vielfältig & $20 \mathrm{MHz}$ & $\pm 50 \mathrm{ppm}$ \\
\hline VCXO & Spannungsgesteuert & Messgeräte & $10 \mathrm{MHz}$ & $\pm 50 \mathrm{ppm}$ \\
\hline TCXO & Temperaturkompensiert & Messgeräte & $10 \mathrm{M} \mathrm{Hz}$ & $\pm 1 \mathrm{ppm}$ \\
\hline OCXO & Ofenkontrolliert & Medizin (MRT) & $10 \mathrm{M} \mathrm{Hz}$ & $\pm 3 \mathrm{ppb}$ \\
\hline
\end{tabular}

Bei VCXO-Quarzoszillatoren (engl.: Voltage Controlled Crystal Oscillator) besteht die Möglichkeit, je nach Anwendungsgebiet, die Resonanzfrequenz in einem bestimmten Bereich durch Anlegen einer Gleich- bzw. Wechselspannung zu steuern. Allgemein kann die Oszillatorfrequenz durch den mit dem Schwingquarz in Reihe geschalteten Lastkondensator $C_{L}$ verändert werden (vgl. Abschnitt B.2). Ersetzt man den Lastkondensator $C_{L}$ durch eine Kapazitätsdiode ist es möglich, durch die Vorspannung an der Kapazitätsdiode die Nennfrequenz des Oszillators zu steuern. Typische Werte liegen bei \pm 100 ppm [164]. Im einfachsten Fall kann durch Anlegen einer veränderbaren Gleichspannung die Oszillatorfrequenz nachjustiert werden.

Bei TCXO-Quarzoszillatoren (engl.: Temperature Compensated Crystal Oscillator) wird die Kompensation des Temperaturganges durch die temperaturabhängige Ansteuerung eines VCXO realisiert. Die Temperatur des Quarzes wird durch einen Temperaturfühler ermittelt. Eine elektronische Schaltung wertet den Temperaturfühler aus und steuert die Kapazitätsdiode so an, dass die Einwirkungen durch den Temperaturgang kompensiert werden. Je nach Aufbau der Schaltungsart unterscheidet man in analoge oder digitale TCXO-Oszillatoren. TCXO-Quarzoszillatoren werden in hermetisch dichten Gehäusen integriert und sind wärmetechnisch zur Umgebung hin isoliert, damit plötzliche Temperaturschwankungen keinen unmittelbaren Einfluss ausüben können.

OCXO-Quarzoszillatoren (engl.: Oven Controlled Crystal Oscillator) werden dort eingesetzt, wo sehr hohe Anforderungen an die Frequenzgenauigkeit gestellt werden. Die stabile Frequenzcharakteristika wird zum einen erreicht durch Verwendung eines Quarzes mit SC-Schnitt, zum anderen durch die Verwendung von Heizelementen, die den Quarz auf eine konstant definierte Betriebstemperatur aufheizen. Die Betriebstemperatur wird dabei auf den optimalen Arbeitspunkt in der Frequenz-TemperaturKennlinie des Quarzes bzw. seines Schnittwinkels bezogen.

Zusätzlich muss die Temperatur auch noch deutlich über der höchsten Betriebstemperatur des Gerätes liegen. Durch die richtige Wahl des Quarzbetriebspunktes werden die Abhängigkeit von der Umgebungstemperatur und der Einfluss der FrequenzTemperatur-Kennlinie minimiert. Alle Elemente zur Regelung der Betriebstempera- 
tur des Quarzes wie Temperaturfühler, Thermostatkörper, Thermostat und Regelschaltung für die Heizung sind in einem Thermogehäuse hermetisch abgeschlossen. Nachteil ist, dass bei höheren Betriebstemperaturen Alterungsprozesse des Quarzes stärker zu Buche schlagen, außerdem benötigt der OCXO-Quarzoszillator zum Erreichen der hohen Ganggenauigkeit eine Einlaufzeit bzw. eine Aufheizzeit. 



\section{Parameter und Kennwerte der Regelung}

\section{C.1. Modellparameter der Regelung}

Parameter der Regelstrecke:

Statorwiderstand:

Rotorinduktivität bezogen auf $d$-Achse:

Rotorinduktivität bezogen auf $q$-Achse:

Polpaarzahl:

Verketteter Erregerfluss:

Wechselrichtertotzeit:

Maximalspannung des Wechselrichters:

Trägheitsmoment des Motors:

Trägheitsmoment der Last:

Reglerparameter PI-Vergleichsregler:

Zeitkonstante Strom-Messfilter:

Zeitkonstante Drehzahl-Messfilter:

Reglerparameter der LPMC-Regelung:

Abtastzeit:

Maximalstrom des Motors:

Maximaldrehzahl des Motors:

Maximalmoment des Motor:

Gewichtungsfaktor $d$-Strom:

Gewichtungsfaktor $q$-Strom:

Gewichtungsfaktor Drehzahl:

Gewichtungsfaktor Position:

Gewichtungsfaktor Änderung $d$-Spannung:

Gewichtungsfaktor Änderung $q$-Spannung:

$$
\begin{aligned}
& R_{s}=1.07 \Omega \\
& L_{d}=4.8 \cdot 10^{-3} \mathrm{H} \\
& L_{q}=4.8 \cdot 10^{-3} \mathrm{H} \\
& p=5 \\
& \psi_{P M}=0.1448 \mathrm{Vs} \\
& T_{t}=1 /\left(16 \cdot 10^{3}\right) \mathrm{s} \\
& U_{W, \max }=326 \mathrm{~V} \\
& J_{M}=3.37 \cdot 10^{-4} \mathrm{~kg} \mathrm{~m}^{2} \\
& J_{L}=30 \cdot 10^{-4} \mathrm{~kg} \mathrm{~m}^{2}
\end{aligned}
$$

$$
\begin{aligned}
& T_{F i}=10^{-3} s \\
& T_{F \omega}=10^{-2} s
\end{aligned}
$$

$$
\begin{aligned}
& T_{S}=10^{-3} s \\
& I_{M, \max }=40.5 \mathrm{~A} \\
& \omega_{M, \max }=4000 \frac{2 \pi}{60} \frac{1}{\mathrm{~s}} \\
& M_{M, \max }=38 \mathrm{Nm} \\
& w_{i, d}=1 \\
& w_{i, q}=0 \\
& w_{\omega}=10 \\
& w_{\vartheta}=0 \\
& w_{u, d}=1 \\
& w_{u, q}=1
\end{aligned}
$$




\section{C.2. Kennwerte der Kaskadenregelung}

Nachfolgend sind die Übertragungsfunktionen der PID-Kaskadenregelung für die im Abschnitt 6.3.4 eingesetzte Vergleichsregelung zusammengestellt. Die Grundeinstellung der Stromregler $G_{R i, j}(s)$ im $d$ - und $q$-Zweig erfolgt nach dem Kriterium des Betragsoptimums gemäß den Gln. (5.32) und (5.33) für die Werte $T_{n i}$ und $K_{P i}$. Die Grundeinstellung des Drehzahlreglers $G_{R \omega}(s)$ erfolgt nach dem Kriterium des symmetrischen Optimums gemäß den Gln. (5.36) und (5.37) für die Werte $T_{n \omega}$ und $K_{P \omega}$.

Die Messwertfilter für die Ströme $G_{F i, j}(s)$ und die Drehzahl $G_{F \omega}(s)$ sind als $\mathrm{PT}_{1^{-}}$ Glied berücksichtigt. Um ein zu starkes Überschwingen bei Führungsgrößenänderungen zu vermeiden, ist der Führungsgrößenfilter $G_{\omega}(s)$ integriert.

Übertragungsfunktion der Stromregler:

$$
G_{R i, j}(s)=K_{P i, j} \frac{1+s T_{n i, j}}{s T_{n i, j}}, \quad j \in\{d, q\}
$$

Übertragungsfunktion des Drehzahlreglers:

$$
G_{R \omega}(s)=K_{P \omega} \frac{1+s T_{n \omega}}{s T_{n \omega}}
$$

Messwertfilter für die Strommessung:

$$
G_{F i, j}(s)=\frac{i_{j, F}}{i_{j, \text { mess }}}=\frac{1}{1+T_{F i} s}, \quad j \in\{d, q\}
$$

Messwertfilter für die Drehzahl:

$$
G_{F \omega}(s)=\frac{\omega_{F}}{\omega_{m e s s}}=\frac{1}{1+T_{F \omega} s}
$$

Führungsgrößenfilter für die Drehzahl:

$$
G_{\omega}(s)=\frac{\omega_{\text {soll }}}{\omega_{\text {soll }, F}}=\frac{1}{1+4\left(T_{F \omega}+2\left(T_{t}+T_{F i}\right)\right) s}
$$




\section{C.3. Modulblöcke der Regelstrecke}

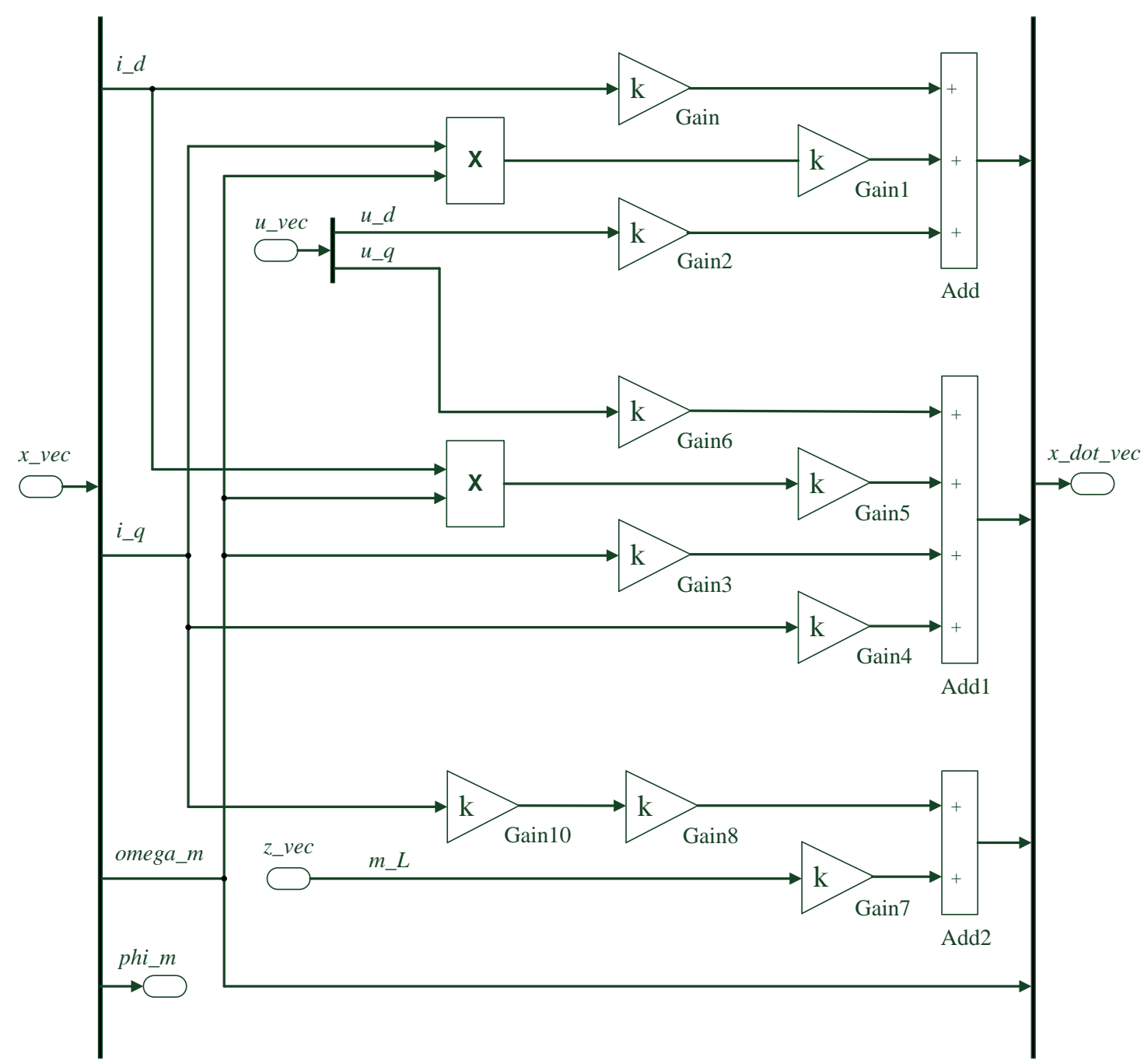

Abbildung C.1.: Blockschaltbild des nichtlinearen Zustandsraummodells. 


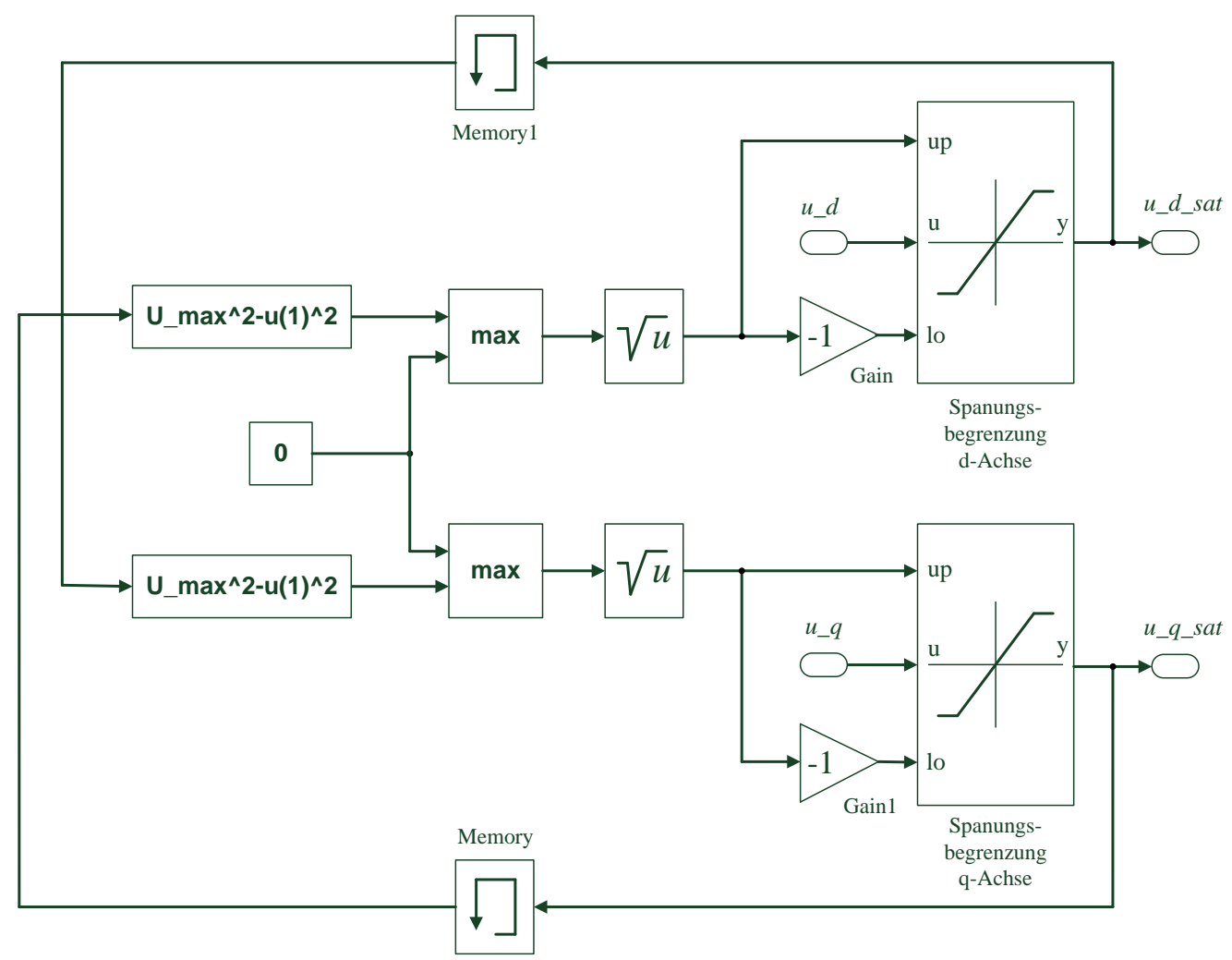

Abbildung C.2.: Blockschaltbild der Spannungsbegrenzung. 


\section{Bezeichnungen}

\section{Allgemeine Schreibweise}

Skalare, Laufvariable

Spaltenvektoren

Zeilenvektoren

Matrizen

Übertragungsfunktionen

Änderung einer Größe
Darstellung in Kursivschrift

Kleinbuchstaben in Fettschrift

Kleinbuchstaben in Fettschrift

Großbuchstaben in Fettschrift

Kursiv in Großbuchstaben

vorangestelltes $\Delta$
z.B. $x$
z.B. $\mathbf{u}$
z.B. $\mathbf{v}^{T}$
z.B. A
z.B. $G(s)$
z.B. $\Delta u$

\section{Indizes}

$\begin{array}{ll}a, b, c & \text { Strangbezeichnungen } \\ \alpha, \beta & \text { Komponenten in Statorkoordinaten } \\ d, q & \text { Komponenten in Rotorkoordinaten } \\ s & \text { Kennzeichnung bzgl. Stator oder Statorkoordinaten } \\ r & \text { Kennzeichnung bzgl. Rotor oder Rotorkoordinaten } \\ * & \text { Darstellung einer Führungsgröße } \\ \sim & \text { Darstellung einer prädizierten Größe } \\ - & \text { Schätzwert einer Variablen }\end{array}$

\section{Griechische Symbole}

$\Gamma$

$\epsilon(k)$

$\epsilon_{r}$

$\epsilon(t)$

$\theta(t)$

$\lambda$

$\Lambda$

$\mu_{r}$

$\xi(k)$

$\sigma^{2}$

$\tau$

$\tau_{0}$

$\varphi_{0}$

Gewichtsmatrix zur Bestimmung des Gütekriteriums

Prädiktionsfehler

relative Permittivität

Amplitudenschwankungen des Spannungssignals

Phasenlage des Sendesignals

Gewichtsfaktor für $\Delta u$

Gewichtsmatrix zur Bestimmung des Gütekriteriums

relative Permeabilität

zeitdiskrete Rauschgröße

Varianz für das Phasenrauschen eines Oszillators

Messzeit zur Darstellung der Varianz

Zeitintervall für die Messungen

Phasenlage des Spannungssignals 


$\begin{array}{ll}\Delta & \text { zeitdiskreter Differenzieroperator } \\ \Delta \varphi(t) & \text { Phasenschwankungen des Spannungssignals } \\ \psi_{P M} & \text { Rotorfluss der PMSM } \\ \psi_{P M \alpha} & \text { Rotorfluss bezogen auf die } \alpha \text {-Achse } \\ \psi_{P M \beta} & \text { Rotorfluss bezogen auf die } \beta \text {-Achse } \\ \psi_{r d} & \text { Rotorfluss bezogen auf die } d \text {-Achse } \\ \psi_{r q} & \text { Rotorfluss bezogen auf die } q \text {-Achse } \\ \psi_{r}^{r} & \text { Rotorfluss bezogen auf Rotorkoordinaten } \\ \psi_{r}{ }^{s} & \text { Rotorfluss bezogen auf Statorkoordinaten } \\ \psi_{s}^{s} & \text { Statorfluss bezogen auf Statorkoordinaten } \\ \omega_{e l} & \text { elektrische Winkelgeschwindigkeit } \\ \omega_{m e c h} & \text { mechanische Winkelgeschwindigkeit } \\ \omega_{M, \max } & \text { Maximaldrehzahl des Motors }\end{array}$

\section{Formelzeichen}

$\begin{array}{ll}\mathbf{a} & \text { komplexer Drehzeiger } \\ \mathbf{A} & \text { Systemmatrix } \\ a_{1}, a_{2}, a_{3}, a_{4} & \text { Berechnung der Randkomponenten des Spannungsvektors } \\ a(n) & \text { Sequenz der zu übertragenden binären Datenbit } \\ \tilde{a}(n) & \text { empfangene Datenbit } \\ a(t) & \text { Sendeamplitude des modulierten Signals } \\ A_{\text {Reg }} & \text { Registereingang der ALU } \\ a_{T} & \text { Koeffizient für den Temperaturverlauf } \\ A\left(z^{-1}\right) & \text { Polynome in } z^{-1} \\ \mathbf{b} & \text { Eingangsvektor } \\ b(n) & \text { paralleler Datenstrom } \\ \mathbf{B}_{d} & \text { Eingangsmatrix der nicht messbaren Störungen } \\ B_{\text {Reg }} & \text { Registereingang der ALU } \\ b_{T} & \text { Koeffizient für den Temperaturverlauf } \\ \mathbf{B}_{u} & \text { Eingangsmatrix } \\ \mathbf{B}_{v} & \text { Eingangsmatrix der messbaren Störungen } \\ B\left(z^{-1}\right) & \text { Polynome in } z^{-1} \\ \mathbf{c} & \text { Ausgangsvektor } \\ \mathbf{C} & \text { Ausgangsmatrix } \\ c_{0} & \text { Lichtgeschwindigkeit } \\ C_{D} & \text { dynamische Kapazität } \\ C_{G} & \text { Kapazität des Gyrators } \\ C_{L} & \text { Lastkapazität } \\ c_{m e d i u m} & \text { Ausbreitungsgeschwindigkeit elektromagnetischer Wellen } \\ C_{S} & \text { statische Kapazität } \\ c_{T} & \text { Koeffizient für den Temperaturverlauf }\end{array}$




\begin{tabular}{ll}
$C\left(z^{-1}\right)$ & Polynome in $z^{-1}$ \\
$C_{Z K}$ & Zwischenkreiskondensator \\
$d$ & Dämpfung \\
$d(k+j \mid k)$ & Störgröße für den Zeitpunkt $k+j$, berechnet zum Zeitpunkt $k$ \\
$\mathbf{D}_{d}$ & Durchgangsmatrix der nicht messbaren Störungen \\
$\mathbf{d}_{k}$ & Vektor der nicht messbaren Störungen \\
$\mathbf{D}_{v}$ & Durchgangsmatrix der messbaren Störungen \\
$e(t)$ & Differenzsignal vor und nach der Begrenzung \\
$E_{R o t}$ & Rotationsenergie \\
$E_{Z K}$ & Energie im Zwischenkreis \\
$f$ & Frequenz \\
$\mathbf{F}$ & Prädiktionsmatrix für die freie Prozessantwort \\
$f(k)$ & k-te gemessene Oszillatorfrequenz \\
$f(k+1)$ & nachfolgende Stichprobe der gemessenen Frequenz \\
$f_{0}$ & Nominalfrequenz des Oszillators \\
$f_{B}$ & Bandbreite, Kanalbandbreite \\
$\Delta f$ & Unterträgerabstand \\
$f_{D}$ & Dezimiererausgangsfrequenz \\
$\mathbf{f}_{D}$ & Prädiktionsvektor für die freie Prozessantwort \\
$f_{E T}$ & Frequenz des Einträgersystems \\
$f_{g}$ & Mittenfrequenz der Träger \\
$F_{G m a x}$ & maximaler Fehler der Ganggenauigkeit \\
$f_{I}$ & Informationsfrequenz des modulierten Signals \\
$f_{L}$ & Lastresonanzfrequenz \\
$f_{N}$ & Nennfrequenz \\
$f_{O b e r}$ & Oberschwingungen eines Signales \\
$f_{S}$ & Abtastfrequenz \\
$f_{S e r}$ & Resonanzfrequenz des Serienschwingkreises \\
$f_{\Sigma \Delta}$ & Modulator-Taktfrequenz \\
$f_{T}$ & Trägerfrequenz des modulierten Signals \\
$\Delta f_{T}$ & Frequenzänderung über der Temperatur \\
$G_{F i}(s)$ & Übertragungsfunktion des Strom-Messwertfilters \\
$G_{F \omega}(s)$ & Übertragungsfunktion des Drehzahl-Messwertfilters \\
$G_{o i}(s)$ & Übertragungsfunktion des offenen Stromregelkreises \\
$G_{o \omega}(s)$ & Übertragungsfunktion des offenen Drehzahlregelkreises \\
$G_{R i, j}(s)$ & Übertragungsfunktion des Stromreglers im $d-$ und $q$-Zweig \\
$G_{R \omega}(s)$ & Übertragungsfunktion des Drehzahlreglers \\
$G_{\omega}(s)$ & Übertragungsfunktion des Führungsgrößenfilters \\
$\mathbf{H}$ & Prädiktionsmatrix \\
$\mathcal{H}$ & Hilbert-Transformation \\
$H\left(e^{j \omega}\right)$ & Filterfrequenzgang \\
$h(i)$ & Gewichte der Übergangsfunktion \\
& \\
& \\
\hline &
\end{tabular}




\begin{tabular}{|c|c|}
\hline$h(m)$ & Anfangsbedingung \\
\hline$H(z)$ & Filterübertragungsfunktion \\
\hline$H_{C}$ & Steuerhorizont \\
\hline$H_{\text {Kanal }}(f)$ & Kanalübertragungsfunktion im Frequenzbereich \\
\hline$H_{P}$ & Vorgabe des Prädiktionshorizontes \\
\hline$i, \mathbf{i}_{s}$ & Strom, Stromraumzeiger \\
\hline$i_{a}, i_{b}, i_{c}$ & Strangströme \\
\hline$i_{\alpha}, i_{\beta}$ & Stromkomponenten in $\alpha, \beta$ Koordinaten \\
\hline$i_{\alpha}(s), i_{\beta}(s)$ & Laplace-Transformierte von $i_{\alpha}$ und $i_{\beta}$ \\
\hline$i_{d}, i_{q}$ & Stromkomponenten in $d, q$ Koordinaten \\
\hline$i_{d}(s), i_{q}(s)$ & Laplace-Transformierte von $i_{d}$ und $i_{q}$ \\
\hline$I_{\text {eff }}$ & effektiver Strom des Linienleiters \\
\hline$I_{G}$ & Gyrator-Ausgangsstrom \\
\hline$I_{L}$ & Linienleiterstrom \\
\hline$I_{M, \max }$ & Maximalstrom des Motors \\
\hline$\Im\{\mathbf{x}\}$ & Imaginärteil des komplexen Wertes x \\
\hline $\mathbf{i}^{r}$ & Stromraumzeiger bezogen auf Rotorkoordinaten \\
\hline $\mathbf{i}^{s}$ & Stromraumzeiger bezogen auf Statorkoordinaten \\
\hline$J$ & Axiales Massenträgheitsmoment des Rotors \\
\hline$J(\Delta u)$ & Gütekriterium \\
\hline$J_{g e s}$ & Gesamtmassenträgheitsmoment \\
\hline$J_{L}$ & Massenträgheitsmoment der Last \\
\hline$J_{M}$ & Massenträgheitsmoment des Motors \\
\hline$k$ & zeitdiskreter Zählindex \\
\hline $\mathbf{k}_{1}$ & komplexer Verstärkungsfaktor \\
\hline $\mathbf{k}_{2}$ & komplexer Rückkopplungsfaktor \\
\hline$K_{I i}$ & Verstärkung im Integral-Zweig des PI-Stromreglers \\
\hline$K_{I \omega}$ & Verstärkung im Integral-Zweig des PI-Drehzahlreglers \\
\hline$K_{m}$ & Ordnung des $\Sigma \Delta$-Modulators \\
\hline$K_{P i}$ & Verstärkung im Proportionalteil-Zweig des PI-Stromreglers \\
\hline$K_{P \omega}$ & Verstärkung im Proportionalteil-Zweig des PI-Drehzahlreglers \\
\hline$K_{R W}$ & Verstärkung des zurückgeführten Signales \\
\hline $\mathbf{k}_{S}$ & komplexe Schleifenverstärkung \\
\hline$K_{T}$ & Drehmomentkonstante \\
\hline $\mathbf{k}_{V}$ & komplexe Gesamtverstärkung \\
\hline$K_{V \omega}$ & P-Verstärkung der Beschleunigungs-Vorsteuerung \\
\hline$K_{w}$ & Verstärkungsfaktor \\
\hline$L_{1}, L_{2}, L_{3}$ & Außenleiterspannungen \\
\hline$L_{d}, L_{q}$ & Induktivitäten der PMSM in $d, q$ Koordinaten \\
\hline$L_{s}$ & Statorinduktivität der PMSM \\
\hline$L_{T}$ & Induktivität (Trägheit der Masse) \\
\hline$M$ & Dezimierungsfaktor \\
\hline
\end{tabular}




\begin{tabular}{|c|c|}
\hline$m_{L}$ & Lastmoment \\
\hline$m_{M}$ & Motormoment \\
\hline$M_{M, \max }$ & Maximalmoment des Motor \\
\hline$n$ & Drehzahl \\
\hline$N$ & Anzahl der Unterkanäle und Periodendauer \\
\hline$N_{1}$ & unterer Prädiktionshorizont \\
\hline$N_{2}$ & oberer Prädiktionshorizont \\
\hline$n_{\text {ist }}$ & Istdrehzahl \\
\hline$N_{M}$ & Anzahl der Messwerte \\
\hline$n_{M P C}$ & Drehzahlverlauf der prädiktiven Regelung \\
\hline$n_{P I D}$ & Drehzahlverlauf der PID-Regelung \\
\hline$N_{u}$ & obere Grenze des Steuerhorizonts \\
\hline$n_{w}$ & Drehzahlsollwert \\
\hline$p$ & Polpaarzahl \\
\hline$P_{c}$ & Leistung, bezogen auf das Frequenzspektrum \\
\hline$p_{d}$ & Bitbreite des Datenwortes \\
\hline$P_{\max }$ & kurzzeitige Leistung des Bremswiderstandes \\
\hline$P_{N}$ & Leistung, bezogen auf das Phasenrauschen im Spektrum \\
\hline Q & Gewichtsmatrix \\
\hline$Q_{I} \ldots Q_{I V}$ & Quadranten der Raumzeigerdarstellung \\
\hline$Q(f)$ & Rauschübertragungsfunktion \\
\hline$q_{n}$ & Anzahl der Bit für den n-ten Datenstrom \\
\hline$Q_{S}$ & Schwingungsgüte eines Oszillators \\
\hline $\mathbf{R}$ & Gewichtsmatrix \\
\hline$r(t)$ & Empfangssignal \\
\hline$R_{B W}$ & Bremswiderstand \\
\hline$r_{d}(k)$ & diskrete Empfangssignalfolge im Zeitbereich \\
\hline$R_{R}$ & Resonanzwiderstand \\
\hline$R_{s}$ & ohmscher Statorwiderstand der PMSM \\
\hline$\Re\{\mathbf{x}\}$ & Realteil des komplexen Wertes x \\
\hline$s$ & Laplace-Faktor \\
\hline $\mathrm{S}_{1}, \mathrm{~S}_{2}, \mathrm{~S}_{3}$ & Schalter (Kombinationen zur Darstellung der Raumzeiger) \\
\hline$S_{1} \ldots S_{6}$ & Sektoren der Raumzeigerdarstellung \\
\hline$s_{d}(k)$ & diskrete Sendesignalfolge im Zeitbereich \\
\hline $\mathbf{s}(f)$ & komplexes frequenzabhängiges Quellensignal \\
\hline$S_{\text {ist }}$ & Istposition \\
\hline$S_{\text {soll }}$ & Sollposition \\
\hline$s(t)$ & Sendesignal \\
\hline$T$ & Umgebungstemperatur \\
\hline$T_{0}$ & Bezugstemperatur \\
\hline$t_{1}$ & Startzeit Sync-Telegramm Masteruhr \\
\hline$\Delta t_{1}, \Delta t_{2}$ & Differenzzeiten zur Berechnung von Delay und Offset \\
\hline
\end{tabular}




\begin{tabular}{|c|c|}
\hline$T_{1 F T}$ & Teilnehmer 1, Frequenztoleranz des Oszillators \\
\hline$T_{2 F T}$ & Teilnehmer 2, Frequenztoleranz des Oszillators \\
\hline$t_{2}$ & Empfangszeit Sync-Telegramm Slaveuhr \\
\hline$t_{3}$ & Startzeit Delay-Request Telegramm Slaveuhr \\
\hline$t_{4}$ & Empfangszeit Delay-Request Telegramm Masteruhr \\
\hline$T_{d}$ & Zeitkonstante des $\mathrm{PT}_{1}$-Gliedes im $d$-Zweig \\
\hline$t_{D S}$ & Dauer des Schutzintervalls \\
\hline$T_{E i}$ & Ersatzzeitkonstante des Stromregelkreises \\
\hline$T_{E i, F \omega}$ & Ersatzzeitkonstante Stromregelkreis und Drehzahlfilter \\
\hline$T_{E T}$ & Symbolintervallzeit eines Einzelträgersystems \\
\hline$T_{F i}$ & Zeitkonstante des Stromfilters \\
\hline$T_{F \omega}$ & Zeitkonstante des Drehzahlfilters \\
\hline$T_{\text {Imp }}$ & Testimpuls \\
\hline$T_{l}$ & Schaltzeiten des linken Randvektors \\
\hline$t_{L}$ & Laufzeitzähler \\
\hline$\Delta t_{n}$ & Zeitintervall \\
\hline$T_{n i}$ & Nachstellzeit des PI-Stromreglers \\
\hline$T_{n \omega}$ & Nachstellzeit des PI-Drehzahlreglers \\
\hline$T_{p}$ & Pulsperiode eines PWM-Signales \\
\hline$T_{P}$ & Prädiktionszeitraum \\
\hline$T_{p}^{*}$ & Hälfte der Pulsperiode eines PWM-Signales \\
\hline$T_{q}$ & Zeitkonstante des $\mathrm{PT}_{1}$-Gliedes im $q$-Zweig \\
\hline$T_{r}$ & Schaltzeiten des rechten Randvektors \\
\hline$T_{S}$ & Sampling bzw. Abtastzeit \\
\hline$t_{\text {Sig }}$ & Signallaufzeit \\
\hline$T_{\Sigma}$ & Verzögerungszeit der Strommessung \\
\hline$T_{S t}$ & Zeitkonstante des Stellgliedes \\
\hline$T_{S t, F i}$ & Ersatzzeitkonstante aus Stelleinrichtung und Stromfilter \\
\hline$T_{s \omega}$ & Zeitkonstante des Führungsgrößenfilters \\
\hline$T_{\text {Sym }}$ & OFDM-Symboldauer \\
\hline$t_{\text {Sym }(e f f)}$ & effektive Symboldauer \\
\hline$\Delta t_{S y n}$ & Zeitdauer bzw. Intervall der Synchronisation \\
\hline$T_{t}$ & Summarische Totzeit der Stelleinrichtung \\
\hline$t_{U h r}$ & aktuelle Uhrzeit \\
\hline$t_{U h r_{k}}$ & korrigierte Uhrzeit \\
\hline$T_{V \omega}$ & Zeitkonstante für die Beschleunigungs-Vorsteuerung \\
\hline$T_{w}$ & elektrische Zeitkonstante \\
\hline$t_{Z}$ & aktueller Zeitstempel \\
\hline$u, \mathbf{u}$ & Spannung, Spannungsraumzeiger \\
\hline$\Delta u, \Delta \mathbf{u}$ & Stellgrößenänderung, Stellgrößenänderungsvektor \\
\hline$u, v, w$ & Phasenstränge \\
\hline$U_{0}$ & Amplitude des Spannungssignals \\
\hline
\end{tabular}




\begin{tabular}{|c|c|}
\hline $\mathbf{u}_{0} \ldots \mathbf{u}_{7}$ & Raumzeiger zur Erzeugung der Ausgangsspannungen \\
\hline$u_{1}$ & Eingangssignal der Schaltung \\
\hline$u_{2}$ & Eingangssignal des Verstärkers \\
\hline$u_{3}$ & Ausgangssignal des Verstärkers \\
\hline$u_{4}$ & Rückgekoppeltes Signal \\
\hline$u_{a}, u_{b}, u_{c}$ & Strangspannungen \\
\hline$U_{A}$ & Ausgangsspannung \\
\hline$U_{A m p}$ & Amplitude Testsimpuls \\
\hline$U_{A S}$ & Ausgangsspannung des Einspeise-Stellers \\
\hline$u_{\alpha}, u_{\beta}$ & Spannungskomponenten in $\alpha, \beta$ Koordinaten \\
\hline$u_{d}, u_{q}$ & Spannungskomponenten in $d, q$ Koordinaten \\
\hline$u_{d(\text { kopp })}$ & verkoppelte Komponente von $u_{d}$ \\
\hline$u_{d(\operatorname{lin})}$ & linearisierte Komponente von $u_{d}$ \\
\hline $\begin{array}{l}u_{d, w} \\
u(k), \mathbf{u}\end{array}$ & $\begin{array}{l}\text { Spannungskomponente in } d \text { - Richtung mit Totzeit } \\
\text { zeitdiskrete Steuergröße, Steuergrößenvektor }\end{array}$ \\
\hline$\Delta u(k+j)$ & Stellgröße für den Zeitpunkt $k+j$ \\
\hline $\mathbf{u}_{l}$ & linker Spannungsvektor des Raumzeigers \\
\hline$u_{\max }$ & maximal zulässige Steuergröße \\
\hline$\Delta u_{\max }$ & maximal zulässige Steuergrößenänderung \\
\hline$u_{\min }$ & unterere zulässige kleinste Steuergröße \\
\hline$\Delta u_{\min }$ & minimal zulässige Steuergrößenänderung \\
\hline$u_{q, w}$ & Spannungskomponente in $q$ - Richtung mit Totzeit \\
\hline$u_{q(k o p p)}$ & verkoppelte Komponente von $u_{q}$ \\
\hline$u_{q(\text { lin })}$ & linearisierte Komponente von $u_{q}$ \\
\hline$u_{q, M P C}$ & Stell-Spannung im $q$-Zweig der prädiktiven Regelung \\
\hline$u_{q, P I D}$ & Stell-Spannung im q-Zweig der PID-Regelung \\
\hline $\mathbf{u}_{r}$ & rechter Spannungsvektor des Raumzeigers \\
\hline $\mathbf{u}^{r}$ & Spannungsraumzeiger bezogen auf Rotorkoordinaten \\
\hline$U_{r m s}$ & Rauschspannung \\
\hline $\mathbf{u}^{s}$ & Spannungsraumzeiger bezogen auf Statorkoordinaten \\
\hline$U(t)$ & Spannungssignal mit Rauschanteil \\
\hline$U_{u n}, U_{v n}, U_{w n}$ & Phasenspannungen \\
\hline$U_{u v}, U_{v w}, U_{w u}$ & verkettete Spannungen \\
\hline$U_{V}$ & Versorgung Gleichspannungs-Zwischenkreis Verbraucher \\
\hline$U_{W, \max }$ & Maximalspannung des Wechselrichters \\
\hline $\begin{array}{l}U_{Z K} \\
v(k)\end{array}$ & $\begin{array}{l}\text { Zwischenkreisspannung des Umrichters } \\
\text { zeitdiskretes Rauschsignal }\end{array}$ \\
\hline $\mathbf{v}_{k}$ & Vektor der messbaren Störungen \\
\hline$w(k), \mathbf{w}$ & Sollwertvariable, Sollwerttrajektorie \\
\hline$w(k+j)$ & vorgegebener Sollwert für den Zeitpunkt $k+j$ \\
\hline$w(n)$ & Datenstrom am Ausgangs des Tiefpassfilters \\
\hline $\mathbf{x}\left(k T_{S}\right)$ & diskrete Signalfolge im Zeitbereich \\
\hline
\end{tabular}




$\begin{array}{ll}x(k), \mathbf{x} & \text { zeitdiskrete Zustandsgröße, Zustandsvektor } \\ \mathbf{x}\left(n / N T_{S}\right) & \text { diskrete Signalfolge im Frequenzbereich } \\ x(n) & \text { binäres Signal } \\ x_{I}(n) & \text { Imaginärteil der diskreten Signalfolge im Frequenzbereich } \\ \dot{\mathbf{x}}_{l i n} & \text { zeitliche Ableitung des linearisierten Zustandsvektors } \\ x_{\text {max }} & \text { maximal zulässige Zustandsgröße } \\ x_{\text {min }} & \text { unterere zulässige kleinste Zustandsgröße } \\ x_{R}(n) & \text { Realteil der diskreten Signalfolge im Frequenzbereich } \\ y(k), \mathbf{y} & \text { zeitdiskrete Regelgröße, Regelgrößenvektor } \\ \tilde{\mathbf{y}} & \text { prädizierter Regelgrößenvektor } \\ \tilde{y}(k+j) & \text { prädizierte Regelgröße für den Zeitpunkt } k+j \\ \tilde{y}(k+j \mid k) & \text { prädizierte Regelgröße für } k+j \text { berechnet zum Zeitpunkt } k \\ y(m) & \text { Ausgangsdaten } \\ \mathbf{y}(n) & \text { diskrete Signalfolge im Frequenzbereich } \\ \bar{y}_{d} & \text { Mittelwert der Daten aus } y_{d}(k) \\ y_{d}(k) & \text { Mittelwert der aufgenommenen Werte } \\ y_{d a}(k) & \text { Mittelwert zur Bestimmung der Allan-Varianz } \\ \mathbf{y}_{k} & \text { Ausgangsvektor } \\ y_{R}(n) & \text { diskrete Signalfolge im Frequenzbereich (Realteil) } \\ \mathbf{z} & \text { Störgröße } \\ Z_{G} & \text { Gyratorimpedanz } \\ z^{-1} & \text { zeitdiskreter Verschiebeoperator } \\ & \end{array}$

